\title{
Spatiotemporal \\ Control of Light \\ in Turbid Media
}

\section{Jochen Aulbach}

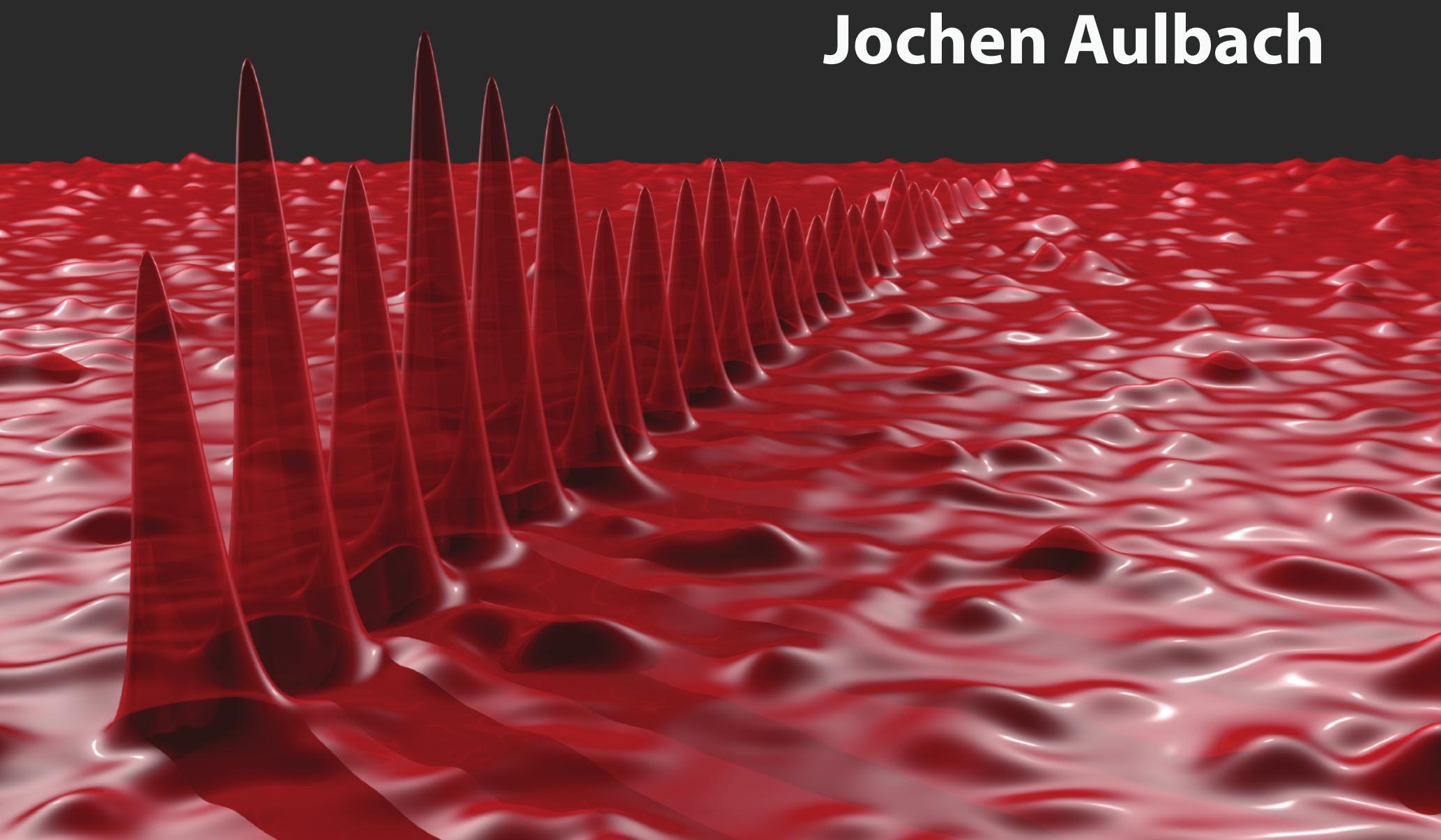




\section{SPATIOTEMPORAL CONTROL OF LIGHT IN TURBID MEDIA}

Beheersing van verstrooid licht in ruimte en tijd 
Promotiecommissie

Promotor

Prof. Dr. A. Lagendijk

Assistent Promotor

Prof. Dr. A. Tourin

Overige leden

Prof. Dr. M. Fink

Prof. Dr. W. L. Vos

Prof. Dr. A. P. Mosk

Prof. Dr. D. Lohse

Paranimfen Dipl.-Phys. L. Langguth

Dr. S. R. Huisman

The work described in this thesis is part of the

Industrial Partnership Programme (IPP)

"Innovatie Physics for Oil and Gas (iPOG)" of the

"Stichting voor Fundamenteel Onderzoek der Materie (FOM)",

which is supported financially by the

"Nederlandse Organisatie voor Wetenschappelijk Onderzoek (NWO)".

The IPP MFCL is co-financed by "Stichting Shell Research".

This work was carried out at the

Center for Nanophotonics, FOM-Institute AMOLF

Science Park 104, 1098 XG Amsterdam, The Netherlands

and the

Institut Langevin, ESPCI ParisTech

Rue Jussieu 1, 75005 Paris, France.

ISBN: 978-90-365-0292-4

This thesis can be downloaded from www.amolf.nl and www.wavefrontshaping.com. 


\title{
SPATIOTEMPORAL CONTROL OF LIGHT IN TURBID MEDIA
}

\section{PROEFSCHRIFT}

\author{
ter verkrijging van \\ de graad van doctor aan de Universiteit Twente, \\ op gezag van de rector magnificus, \\ prof. dr. H. Brinksma, \\ volgens besluit van het College voor Promoties \\ in het openbaar te verdedigen \\ op vrijdag 20 september 2013, om 12.45 uur
}

door

Jochen Aulbach

geboren op 17 augustus 1982

te Groß-Gerau, Duitsland 
Dit proefschrift is goedgekeurd door:

Prof. Dr. A. Lagendijk en Prof. Dr. A. Tourin 


\section{M'illumino \\ d'immenso}

Giuseppe Ungaretti, Mattina 



\section{Contents}

1 Introduction 11

1.1 Spatial control of scattered light . . . . . . . . . . . . . . . 12

1.2 Spatiotemporal control of scattered waves . . . . . . . . . . 13

1.3 Motivation . . . . . . . . . . . . . . . . . . . 14

1.4 Outline of this thesis . . . . . . . . . . . . . . 15

2 Elements of the experimental apparatus $\quad 17$

2.1 Ultrashort pulses - generation and propagation in homogeneous media 18

2.2 Motivation . . . . . . . . . . . . . . . . . . . . 18

2.3 Spatial light modulation of ultrashort pulses . . . . . . . . . . . . . . 19

2.3.1 Spatial modulation of phase, amplitude and polarization . . . 20

2.3.2 Temporal modulation . . . . . . . . . . . . . . . . 21

2.3.3 Spatiotemporal modulation . . . . . . . . . . . . 22

2.3.4 Comparison with other technology . . . . . . . . . . . 22

2.4 Transmission of ultrashort pulses through random media . . . . . . . 22

2.4.1 From waves to the diffusion approximation . . . . . . . . . 23

2.4 .2 Speckle in space and time . . . . . . . . . . . . 26

2.5 Ultrashort pulses - detection . . . . . . . . . . . . . . . . . 32

2.5.1 Spectral and temporal interferometry . . . . . . . . . 32

2.5.2 Autocorrelation ................. 33

2.6 Analogies and differences between optics, microwaves and ultrasound experiments . . . . . . . . . . . . . . . . . 34

2.7 Summary . . . . . . . . . . . . . . . . . . . . 35

3 Control of light transmission through opaque scattering media in space and time

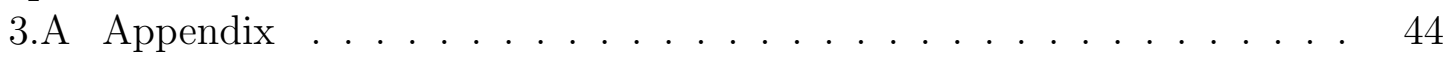

3.A.1 Experiment . . . . . . . . . . . . . . . 44

3.A.2 Duration of the optimized pulses . . . . . . . . . . 45

3.A.3 Influence of noise . . . . . . . . . . . . . . . . . . . . . 49

3.A.4 Enhancement factor and correction for non-uniform illumination of the spatial light modulator . . . . . . . . . . . . . 49

3.A.5 Time-resolved transmission measurements . . . . . . . . . 50

3.A.6 Spatial extent of the focal spot . . . . . . . . . . . 51

4 Analysis of linear and nonlinear feedback methods based on numerical simulations 
4.1 Generation of speckle pulses . . . . . . . . . . . . . . . 55

4.1 .1 Algorithm . . . . . . . . . . . . . . . 55

4.1 .2 Statistical properties . . . . . . . . . . . . . . . . . 57

4.1 .3 Summary and outlook . . . . . . . . . . . . 57

4.2 Short-pulse WFS with a slow nonlinear detector . . . . . . . . . 59

4.2.1 Comparison of linear and nonlinear detection . . . . . . . 59

4.2.2 Pulse duration . . . . . . . . . . . . . . . . 63

4.2 .3 Pulse arrival time . . . . . . . . . . . . . . . . . 64

4.2.4 Conclusions and outlook . . . . . . . . . . . . 68

5 Spatiotemporal focusing in turbid media by wavefront shaping with nonlinear feedback

5.1 Introduction . . . . . . . . . . . . . . . . . . . . . . 69

5.2 Experiment . . . . . . . . . . . . . . . . . . . . . . 71

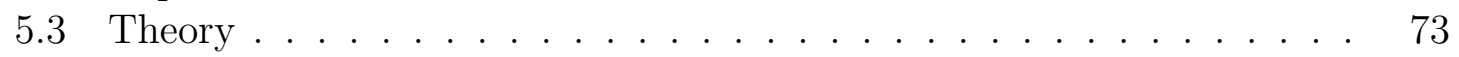

5.3.1 Intensity-intensity autocorrelation of speckle pulses . . . . . . 73

5.3.2 Enhancement of the time-integrated second harmonic intensity 74

5.4 Results and discussion . . . . . . . . . . . . . . . . . . 79

5.4.1 Spatiotemporal focus on a single nanocrystal . . . . . . . . 79

5.4.2 Comparison of the measured and the modeled enhancement . 82

5.4.3 Pulse duration after WFS . . . . . . . . . . . . . 83

5.4.4 Peak-to-background ratio . . . . . . . . . . . . . 84

5.5 Conclusions . . . . . . . . . . . . . . . . . . . 84

5.A Test of noise analysis by simulations . . . . . . . . . . . 86

6 Spatial and temporal focus on nanocrystals hidden deep inside a $\begin{array}{lr}\text { random medium } & 89\end{array}$

6.1 Introduction . . . . . . . . . . . . . . . . . . . . . . . 89

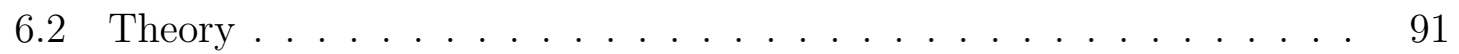

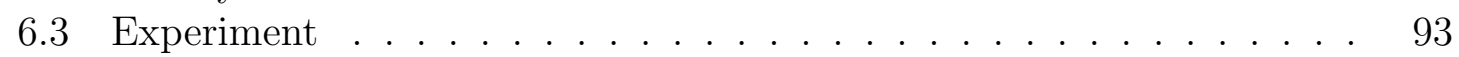

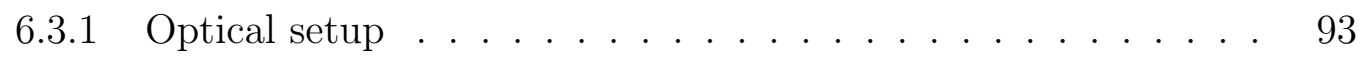

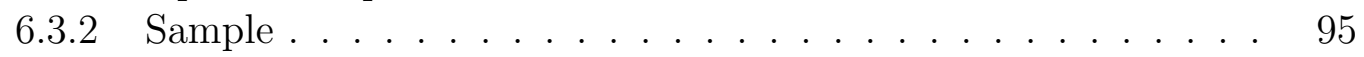

6.3.3 Experimental procedure . . . . . . . . . . . . . 97

6.4 Results . . . . . . . . . . . . . . . . . . . . . . . . . . . 99

6.5 Discussion . . . . . . . . . . . . . . . . . . . . 105

6.6 Conclusions . . . . . . . . . . . . . . . . . 107

7 Optimal spatiotemporal focusing through random media $\quad 109$

7.1 Introduction . . . . . . . . . . . . . . . . . 109

7.2 Wavefront shaping with nonlinear feedback for optimal spatiotemporal focusing . . . . . . . . . . . . . . . . . . 111

7.2 .1 Concept . . . . . . . . . . . . . . . . 111

7.2.2 The matched filter approach for optimal focusing . . . . . . . 111

7.2.3 Detector response in the simulations . . . . . . . . . . . . 113

7.2.4 Steps of the wavefront shaping algorithm . . . . . . . . . . . 113

7.3 Experiment and simulations . . . . . . . . . . . . . . . 118

7.3.1 Transfer matrix measurement . . . . . . . . . . . . 118 
7.3.2 Wavefront shaping simulation . . . . . . . . . . . . . . 119

7.4 Conclusions . . . . . . . . . . . . . . . . . . . . 122

$\begin{array}{ll}\text { Summary } & 123\end{array}$

$\begin{array}{ll}\text { Samenvatting } & 125\end{array}$

$\begin{array}{lr}\text { Zusammenfassung } & 129\end{array}$

$\begin{array}{ll}\text { Acknowledgements } & 133\end{array}$

$\begin{array}{ll}\text { Bibliography } & 136\end{array}$ 



\section{CHAPTER}

\section{Introduction}

Light-based technologies have an increasing impact to virtually all areas of our life and are the basis of a wide range of applications in science and industry. Optical fiber communication systems [1], optical lithography for microfabrication [2], as well as optical microscopy or spectroscopy for medicine and life science research [3, 4] are just a few examples of areas where advanced optical technologies became indispensable. A large part of the fundamental research in the field of optical technologies is about the understanding and the control of light propagation in nanostructured materials [5], with, among other things, the drive towards development of new generations of solar cells [6], computers [7] and imaging techniques [8].

Using advanced optical systems, we can direct light with great accuracy in homogeneous materials, such as glass. On the contrary, in turbid materials, such as biological tissue, even the most perfectly designed lenses or objectives fail to directed light in a controlled manner, due to random scattering in the disordered microscopic structure of the medium. When a beam enters a strongly scattering medium, its nonscattered 'ballistic' component is extinguished exponentially with increasing depth in the medium $[9,10]$. The decay constant is governed by the mean free path, the average distance between two scattering events. Beyond a depth of a few mean free paths, all light is randomized in direction and its transport is usually well-described by diffusion. High-resolution structural information from deeper inside the medium than a couple of mean free path is hidden from outside. Conversely, light cannot be focused inside a random medium by conventional means. Therefore, light scattering by inhomogeneous media has usually been considered a nuisance to applications. 

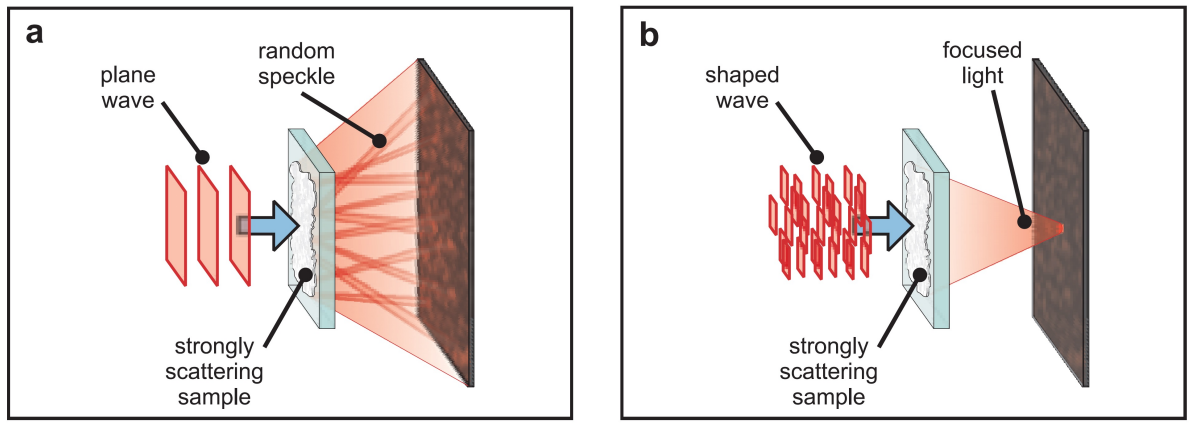

Figure 1.1: Spatial focusing of monochromatic light by wavefront shaping [13]. (a) With conventional illumination by a flat wavefront, a random speckle pattern forms in transmission. (b) The optimal wavefront focuses the light in a tight spot.

\subsection{Spatial control of scattered light}

The development of spatial light modulators (SLMs) has opened the possibility to convert light scattering from a mere impairment into an opportunity for novel approaches to the control of light propagation. SLMs are computer-controlled devices, which spatially control amplitude, phase or polarization of a light wave of a light beam with millions of degrees of freedom [11]. Employed in an optical setup, a SLM can flexibly play the role of various conventional optical elements, such as a scanning mirror, a lens or a grating [12]. Furthermore, SLMs have opened the door for a new class of experiments and applications with light: It is now possible to adapt an optical system in a complexity, which matches the complexity of light scattering by random media.

In their pioneering experiment in 2007, Vellekoop and Mosk showed that multiply scattered light can be controlled through a turbid medium by spatial shaping of the illuminating beam [13]. The experiment is illustrated in Fig. 1.1. For illumination by an unmodulated beam of monochromatic light, a random speckle pattern is observed in transmission. With the optimized wavefront the light is focused behind the medium. This wavefront is found by iterative optimization, employing the intensity in the target spot as feedback signal. Using fluorescence from particles inside the medium as detector, Vellekoop et al. achieved focusing inside a thick scattering layer one year later [14]. The approach, usually termed 'wavefront shaping' (WFS), also gained a wide-spread attention as it allows tests of mesoscopic transport theory with light [15]: In 2008, first WFS experiments gave hints for open transport channels for light through a scattering layer [16].

Stimulated by the first WFS experiments, there has been tremendous progress on many fronts for focusing, imaging, and manipulation by light through random media [17]. Popoff et al. demonstrated that the WFS approach can be parallelized to measure a significant fraction of the medium's transmission matrix [18, 19]. In view of biomedical applications, several research groups have developed techniques for highspeed focusing and transmission matrix measurements through turbid media [20-23]. Van Putten et al. employed WFS to create a scattering lens for high-resolution imaging $[24,25]$. Cui at al. and Hsieh et al. have demonstrated focusing and imaging through scattering layers by digital phase conjugation [26, 27]. Wavefront shaping 

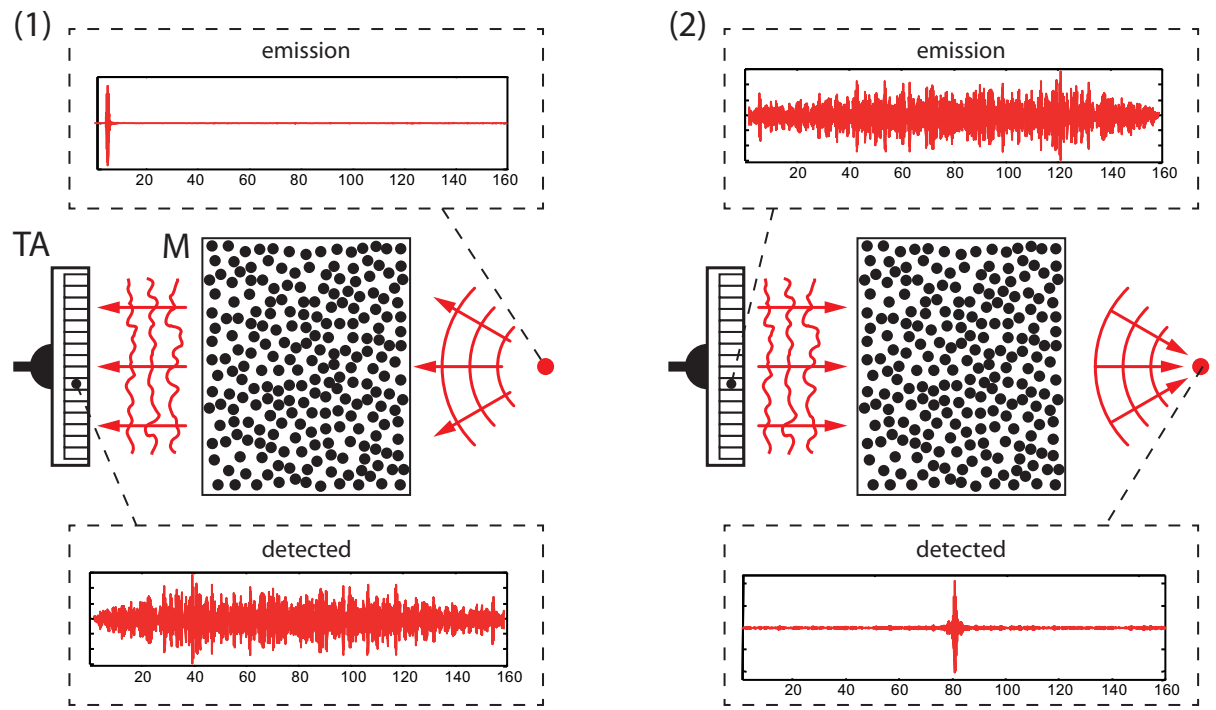

Figure 1.2: Spatial and temporal focusing of ultrasound through random media by time reversal. (1) In the first step, a short pulse is emitted from the source. The waves propagate through the random medium (M) and are detected at the transducer array (TA). (2) In the second step, the time-reversed signals are remitted from the array. Due to reciprocity, the waves refocus in a short pulse at the initial source position.

is also relevant to communication and imaging through fibers: Di Leonardo et al., followed by other research groups, have developed techniques for the transmission of images and holograms through multi-mode fibers [28-30]. Gjonaj et al. transferred the WFS approach to surface plasmon polaritons, which has potential for novel microscopy techniques with subwavelength resolution [31-33]. Jang et al. demonstrated that WFS can improve optical coherence tomography [34]. A very promising approach is the combination of different types of waves: $\mathrm{Xu}$ and coworkers tagged scattered light by an ultrasound focus to create the feedback signal for focusing and imaging through an opaque screen $[35,36]$. In recent exciting experiments, Bertolotti et al. demonstrated reference-free fluorescence imaging through scattering layers [37].

\subsection{Spatiotemporal control of scattered waves}

For acoustic waves, focusing through random media was already demonstrated more than a decade earlier by Fink and coworkers [38]. In contrast to the initial approaches in WFS mentioned above which employ monochromatic light, these ultrasound experiments focus broadband acoustic waves both in space and time: At ultrasound frequency, broadband waves can be both emitted and detected in a single element piezoelectric transducer. When these transducers are combined to 100 element arrays, they can be employed as 'time reversal mirror'. The focusing scheme is illustrated in Fig. 1.2. In the first step, a source emits a short pulse from the intended focal point. The waves diffuse through a thick scattering medium and are recorded at the time reversal mirror (TRM). A fraction of the waves travels through shorter, rather direct paths through the material, reaching the TRM much earlier than pulses that are coupled to very long paths. Because this spread in the arrival times is many 
times larger than the original pulse duration, the signal is not only spatially, but also temporally distorted: The pulses are randomly spread over a wide interval. In the second step, the recorded signal is re-emitted chronologically reversed. Due to reciprocity of the propagation in the scattering medium, the waves recombine to a short pulse at the point of the source [39]. Such time reversal experiments were also the first to demonstrate that scattering by the medium can be used to effectively increase the numerical aperture of the system, leading to a smaller focus compared to the situation without the medium [40]. In 2004, Lerosey et al. translated time reversal focusing to electromagnetic waves at microwave frequencies [41]. In a later study, they placed the source antenna inside a scattering medium, leading to the microwave focus far below free-space diffraction limit [42]. Enabling the focusing of broadband waves both in space and in time through random media, time reversal focusing of sound and microwaves has found a wide range of applications in imaging [43], geophysics [44], medical therapy [45] and communication [46, 47].

\subsection{Motivation}

From an optics point of view, one of the most important outstanding question is whether simultaneous spatial and temporal focusing through and inside random media can be achieved. Many powerful optical techniques require both spatial and temporal concentration of light to either collect information about matter or to modify matter. However, nonlinear spectroscopy [48], multi-photon imaging [49], coherent control [50-52], nanosurgery [53] and nanolithography [54] are by conventional means limited to the ballistic regime in scattering media. Finding novel concepts to control pulsed-light propagation in complex multiple scattering systems is further of high interest in view of applications in designed photonic media and metamaterials for ultrafast switching [55], spontaneous emission control [56] or subwavelength imaging [57].

Since scattering is a general wave phenomenon, concepts and ideas can in principle be translated between any type of acoustic waves and the different regimes of electromagnetic waves. One example is the use of the scattering medium to improve focusing $[40,58]$. However the hardware limitations for generating and detecting acoustic and optical waves can be substantially different. Modulation of broadband signals by a compact element is easy for acoustics and microwaves, but it is difficult to scale this control to many spatial degrees of freedom [17]. The reverse relation is true for light. CCD cameras and SLMs can readily resolve and manipulate many spatial degrees of freedom, but only within a narrow bandwidth. The controlled modulation of coherent broadband light by pulse-shaping techniques [59] requires a relatively extended experimental apparatus even for a single spatial channel. In acoustics and in microwaves, field and amplitude can be measured readily, which for light requires interferometric techniques [60]. A direct equivalent of the time reversal mirror in optics would be desirable. But as a time reversal step requires both an extensive measurement and wavefront synthesis step, a truly practical implementation in optics is beyond reach for the currently available technology. On the other hand, some very versatile tools are restricted to the optics realm: Local intensity probes such particles filled with fluorescent dyes allow the measurement of intensity without 
an electric connection to the detection point and are ideal probes for iterative WFS schemes [14].

\subsection{Outline of this thesis}

In this thesis, we pioneer concepts and experiments for focusing waves both spatially and temporally through random scattering media.

We develop a short-pulse wavefront shaping scheme, demonstrating that the spatial modulation of a short-pulse laser is sufficient to focus pulses through or inside scattering media. We employ our scheme in a series of experiments, demonstrating spatiotemporal focusing of light both through and inside random media.

In chapter 2 we describe all elements of a short-pulse wavefront shaping apparatus which are relevant for this thesis. We discuss the available technologies for the ultrashort pulse generation, modulation and detection mainly from an experimental point of view. A substantial part of the chapter is dedicated to the theoretical description of scattering by random media, providing the theoretical framework for the following chapters.

In chapter 3 we present the first experimental demonstration of spatial and temporal focusing of light through a multiple scattering medium. As feedback signal we use the amplitude of scattered wave field measured at a single point in time and space. Experimentally, this is realized by optical gating with heterodyne interferometric detection. We establish the theoretical framework to explain the achieved pulse duration and the enhancement of the field at the focus. Experiment and theory agree.

Chapters 4, 5 and 6 consecutively build upon one another. In chapter 4 we show that spatiotemporal focusing can be achieved with a slow detector, when the detector response is nonlinear. Based on numerical simulations, we investigate the implications of this approach in terms of the pulse duration at the focus and the control of the pulse arrival time.

The WFS concept based on the slow nonlinear detector is put into experimental practice in chapter 5 . We employ crystalline nanoparticles with a high efficiency for second-harmonic generation (SHG) as nonlinear detectors. We demonstrate spatiotemporal focusing on single nanocrystals which are positioned at the back interface of a scattering slab. In this configuration the SHG signal be can accurately monitored and compared to a thorough model of the experiment. The full power of the approach is demonstrated in chapter 6 . We embed the nanocrystals in a thick scattering slab as a local nonlinear detector. We provide the first experimental demonstration of spatiotemporal focusing deep inside a multiple scattering medium.

In chapter 7 we continue to explore the focusing scheme with a slow nonlinear detector, but our methods differ from the preceding chapters in two ways. First, our findings are based on an ultrasound experiment and second, we investigate broadband wavefront shaping, as ultrasound waves can readily be measured and shaped spatially and temporally. We demonstrate that wavefront shaping of broadband waves can achieve, equivalent to time reversal focusing schemes, optimal focusing. Our findings are based on simulations, which we perform based on an experimentally measured ultrasound transmission matrix of a random medium. 



\section{Elements of the experimental apparatus}

In this chapter we discuss the elements of the apparatus to focus and control short-pulses through random media by wavefront shaping.

Figure 2.1 schematically shows the elements of a wavefront shaping experiment [61] in optics: A laser provides a well-defined coherent beam which is modulated by a wavefront synthesizer. The modulated wavefront is projected onto the random medium which strongly scatters the light. A detection system collects the light scattered from the medium and provides a feedback signal which is used to optimize the wavefront.

In the following we describe one by one the elements of the apparatus for a shortpulse wavefront shaping experiment. We discuss the available technologies for the ultra-short pulse generation, modulation and detection from an experimental point of view. Furthermore, we describe each of elements in the scheme 2.1 analytically, providing the theoretical framework for the simulations in chapter 4 and the modeling and the analysis of the experiments in chapters 3-7. Finally we briefly compare the implementation of WFS experiments in optics with the microwave regime and ultrasound waves.

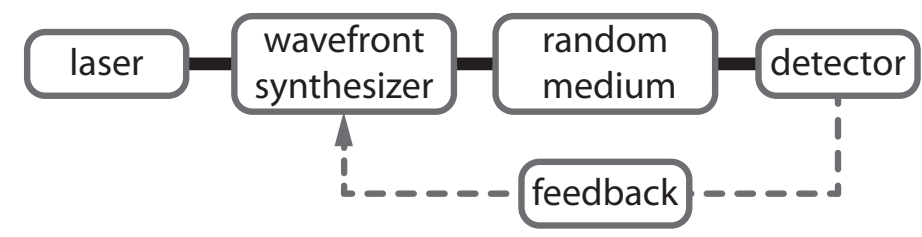

Figure 2.1: Schematic illustration of a wavefront shaping experiment in optics. 

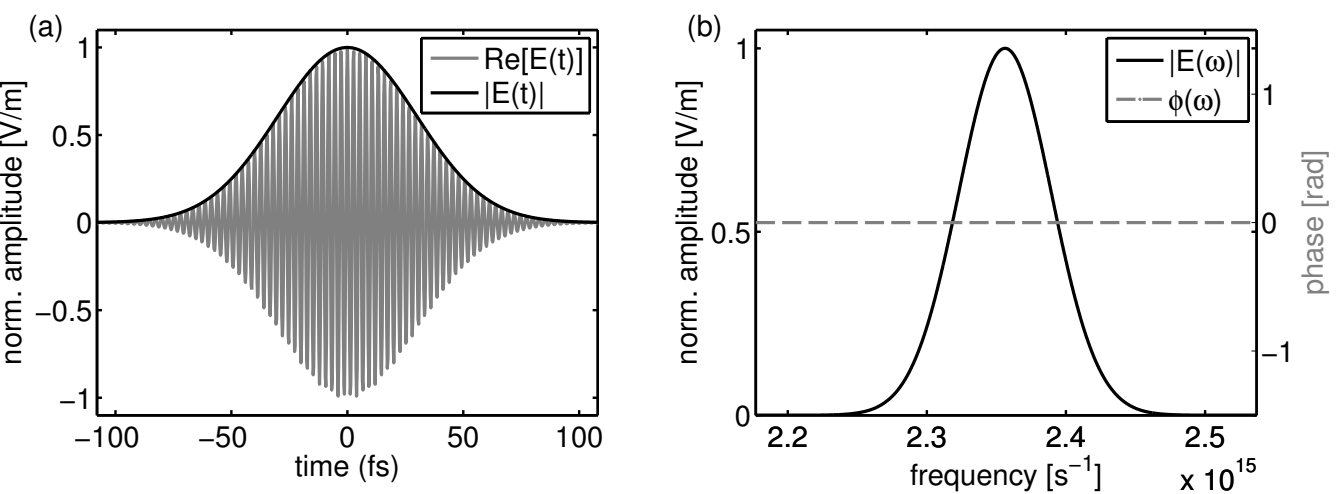

Figure 2.2: Typical ultrashort laser pulse. (a) Real part of the electric field (grey) and its Gaussian-shaped pulse envelope (black) in the time domain for an ultrashort laser pulse with a center wavelength $\lambda=800 \mathrm{~nm}$ and an (intensity) pulse duration $\Delta t=50 \mathrm{fs}$. (b) Corresponding amplitude (black) and phase function (grey) in the frequency domain with a relative bandwidth of the intensity spectrum $\Delta \omega / \omega_{0}=0.024$.

\subsection{Ultrashort pulses - generation and propaga- tion in homogeneous media}

\subsection{Motivation}

Here we give a introduction to the mathematical description of ultra-short laser pulses and introduce related terminology which we will use in later chapters of this thesis. A general and extensive introduction on ultra-short laser pulses can be found in the review by Wollenhaupt et al. [62] and the books by Diels and Rudolph [63] and Rulliere [64].

In general, electromagnetic waves are vector waves. In the following we restrict ourselves to the treatment of scalar waves, as they adequately describe the majority of the phenomena treated in this thesis. The electric field $E(\omega)$ of an ultra-short laser pulse propagating in a single spatial mode is conveniently described by separating the spectral amplitude $|E(\omega)|$ and the phase term $\phi(\omega)$ in polar notation

$$
E(\omega)=|E(\omega)| \mathrm{e}^{-i \phi(\omega)} \underset{\mathcal{F}^{-1}}{\stackrel{\mathcal{F}}{\leftrightarrows}} E(t)=|E(t)| \mathrm{e}^{-i \phi(t)}
$$

The field in the time-domain $E(t)$ is obtained by inverse Fourier transform $\mathcal{F}^{-1}$. The spectral amplitude $|E(\omega)|$ provided by standard Ti:sapphire mode-locked oscillators laser pulses are usually of a Gaussian or sech ${ }^{2}$ shape. The center frequency $\omega_{0}$ of the emission corresponds to a wave-length of approximately $\lambda=800 \mathrm{~nm}$ with a relative bandwidth $\Delta \omega / \omega_{0}$ in the range from $0.02-0.2$. The bandwidth $\Delta \omega$ is by convention the full width at half maximum (FWHM) of the intensity spectrum $I(\omega)$, the pulse duration $\Delta t$ is defined as the FWHM of $I(t)$. A typical ultrashort laser pulse is shown in Fig. 2.2.

To characterize the propagation of the pulse in a homogeneous non-absorbing 
(a)

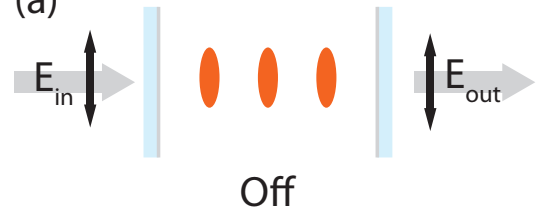

(b)

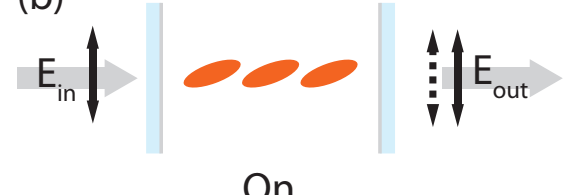

Figure 2.3: Working principle of a parallel-aligned nematic liquid crystal cell for phase-only modulation of light. (a) In the off state, the long axis of the liquid crystals (LC) molecules is oriented parallel to the transparent electrodes. The parallel alignment of the molecules is induced by a line-structure imprinted on the electrodes (not shown). (b) When a voltage is applied, the LC molecules orient along the propagation direction of the light. The refractive index for light polarized parallel to the LC molecules changes from the extraordinary index (no voltage) to the (lower) ordinary index, resulting in phase shift in transmission [11].

medium we expand the phase term $\phi(\omega)$ in a Taylor-series around the center frequency

$$
\phi(\omega)=\phi\left(\omega_{0}\right)+\phi^{\prime}\left(\omega_{0}\right) \cdot\left(\omega-\omega_{0}\right)+\frac{1}{2} \phi^{\prime \prime}\left(\omega_{0}\right) \cdot\left(\omega-\omega_{0}\right)^{2}+\ldots
$$

with

$$
\phi^{(i)}\left(\omega_{0}\right)=\left.\frac{\partial^{(i)} \phi}{\partial \omega^{(i)}}\right|_{\omega_{0}} .
$$

The first term $\phi\left(\omega_{0}\right)$ gives the temporal offset between the pulse envelope and the underlying oscillation with the carrier frequency $\omega_{0}$; it is of no relevance to the experiments presented in this thesis. A positive term $\phi^{\prime}\left(\omega_{0}\right)$ corresponds to a translation of the pulse in the time-domain according to the Fourier-shift theorem. The coefficients of higher order modify the temporal shape of the pulse. The first of these terms is the group velocity dispersion $\phi^{\prime \prime}$. If all higher-order terms are zero, the pulse is bandwidth-limited, i.e., it has the shortest possible pulse duration $\Delta t$ supported by the spectrum. The time-bandwidth product in this limit depends on the spectral shape; for Gaussian pulses it can be calculated as $\Delta t \Delta \omega=4 \ln 2$. A non-zero value $\phi^{\prime \prime}$ corresponds to a temporal broadening of the pulse. When a pulse travels through a medium of thickness $L$, it acquires the group velocity dispersion [62]

$$
\phi^{\prime \prime}=\frac{\lambda^{3} L}{2 \pi c^{2}} \frac{d^{2} n}{d \lambda^{2}}
$$

where $n(\lambda)$ is the index of the medium. Most transparent materials show normal dispersion with $\phi^{\prime \prime}>0$ for visible light, such that the 'red' parts of the laser pulse travel faster through the medium than the 'blue' parts. An often-used terminology is that the pulse obtains a positive 'chirp' on propagation through a medium with normal dispersion. Anomalous dispersion with $\phi^{\prime \prime}<0$ occurs at frequencies at which the radiation is resonantly scattered in a medium, which for most materials typically is the case in the X-ray regime [65].

\subsection{Spatial light modulation of ultrashort pulses}

Spatial light modulation and pulse shaping by programmable optical devices has become practical since the 1990s thanks to developments of liquid crystal arrays 
and deformable mirrors [11]. In this sense the field of wavefront shaping is very much technology-driven. The development of large liquid crystal arrays was strongly motivated by the huge market for display technology.

In Fig. 2.3 we schematically describe the working principle of a pixel of a phaseonly spatial light modulator based on parallel-aligned liquid crystal cells such as we will use it in our experiments. A controlled change of the applied voltage to the cell reorients the LC molecules, leading to a change of the refractive index $\Delta n$. For a layer of thickness $L$ this change consequently leads to a controllable temporal shift $\tau=\Delta n L / c$ for the pulses transmitted through the cell with respect to a reference time. The cells are designed that a temporal delay of up to one optical cycle or more at the center frequency $\tau=\{0 \ldots 2 \pi\} / \omega_{0}$ can be applied:

$$
E(t, \tau)=|E(t-\tau)| e^{-i \phi(t-\tau)} \approx|E(t)| e^{-i \phi(t)} e^{-i \varphi(\tau)} .
$$

In the last step we applied the 'slowly varying envelope approximation' (SVEA): As the bandwidth of typical laser short pulses is relatively narrow, the pulse envelope $|E(t)|$ is constant over the timescale $\tau$ and the phase function $\phi(t)$ has negligible higher-order terms besides the oscillation with the carrier frequency $\omega_{0}$ (see Fig. 2.2). The temporal shift therefore has the same effect as an additional phase factor $\varphi(\tau)=$ $\tau \omega_{0}=\{0 \ldots 2 \pi\}$. The analogous description in the frequency domain follows from the Fourier shift theorem,

$$
E(\omega, \tau)=|E(\omega)| e^{-i \phi(\omega)} e^{-i \tau \omega} \approx|E(\omega)| e^{-i \phi(\omega)} e^{-i \varphi(\tau)} .
$$

The interpretation of the SVEA is as follows: Since the bandwidth of the pulses is narrow with respect to their central frequency $\omega_{0}$, the phase factor $\tau \omega$ is close to constant over the spectral range $\varphi=\tau \omega_{0}$.

\subsubsection{Spatial modulation of phase, amplitude and polariza- tion}

Using a two-dimensional liquid crystal array, a beam from a short-pulse laser source can be spatially modulated (Fig. 2.4). Based on the phase-only modulation technique, the SLM can be programmed to function as a lens, a grating, a stirring mirror, or in our case, to prepare the optimal random wavefront to illuminate a random medium.

A phase modulation is not the only possible means to shape the wavefront. SLMs based on twisted nematic crystals allow amplitude modulation and polarization shaping [66]. Standard back-illuminated liquid crystal displays are amplitude modulators based on twisted nematic liquid crystals: the crystals layer is packed between the electrodes and two cross-polarizers. The crystals turn the polarization by 90 degrees in the off-state and the device is transparent. With increasing voltage applied to the cell the crystals align such that the polarization is turned less and the transmission drops. Without the cross-polarizers, the array acts as a spatial polarization modulator. With a combination of several SLM layers, the simultaneous modulation of amplitude, phase and polarization can be achieved. An elegant way to achieve phase and amplitude modulation on a single SLM is the 4-pixel technique [66].

Apart from our research field, there is a wide range of applications of spatial light modulation by SLMs. As an SLM can modify a laser beam without any moving 
(a)

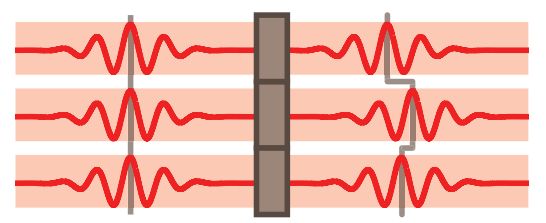

(b)

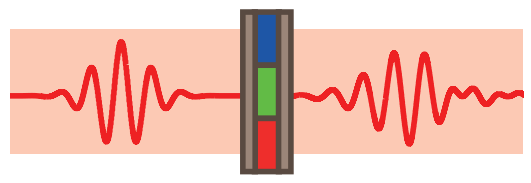

(c)

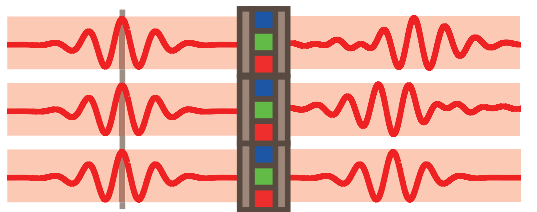

Figure 2.4: Spatial and temporal modulation of light. (a) Short-pulse spatial light modulation: A spatial light modulator delays the pulses with a controlled phase shift individual to each segment. For an illustrative purpose a few-cycle pulse is sketched here. The SVEA used in Eq. 2.4 does not apply here. (b) Pulse shaping: For a single spatial mode, the pulse shaper introduces a specific controlled phase shift for each frequency. (c) Spatiotemporal light modulator by multiplexing of a pulse shaper to control each spatial mode.

mechanic components, it is ideal for beam-stirring for microscopy [67] or structuredillumination microscopy [68]. SLMs provide a convenient means to prepare specific spatial beam profiles such as self-reconstructing Bessel beams [69]. These nondiffracting beams have been implemented to improve microscopy [70] or to control the generation of laser filaments [71].

\subsubsection{Temporal modulation}

Pulse shaping - the modulation of the temporal profile of a laser pulse - was the first application of liquid crystal devices in the field of ultrafast optics (Fig 2.4b) [59]. The temporal modulation of sub-nanosecond pulses can only be realized in the frequencydomain, since the fastest modulations which can be applied to a laser beam directly in the time-domain by means of an electro-optical device are in the $\mathrm{GHz}$ range. The most often used approach is a 4-f pulse shaper: the pulse is transformed to the spectral domain by means of a grating. In the Fourier plane, a liquid crystal linearray modulates the field of each spectral component separately. The components are recombined to form the modulated pulse by another grating or the same grating in a reflective design. In the same fashion as for spatial-domain shaping, amplitude, phase and polarization of each frequency component can be shaped by multi-layer SLMs [72]. An alternative approach for pulse shaping is the use of acousto-optic programmable dispersive filter, which can also be used in the UV range in contrast to LCD-based designs [73]. The most important application of pulse-shapers is coherent control [50], in particular of chemical reactions [74-76]. 


\subsubsection{Spatiotemporal modulation}

Conceptually, the approaches of wavefront shaping and pulse shaping can be easily combined for spatiotemporal pulse shaping (Fig. 2.4c). This ideal shaping device would modulate a wavefront in one temporal and in two spatial dimensions with a high number of degrees of freedom in all dimensions. A 2D spatial light modulator can be used for temporal modulation (in the frequency domain) along one axis and for spatial modulation along the other [77]. If a second spatial dimension is required, a convenient scheme of mapping would need to be designed. The future challenge is to realize the mapping without introducing too many artifacts, which will inevitably occur when conventional optical elements are used.

\subsubsection{Comparison with other technology}

Liquid crystal SLMs for research applications have relatively low purchase costs (around 10k€) and provide a large number of controllable degrees of freedom $\left(\sim 10^{6}\right.$ pixels). Liquid crystal SLMs are limited to frame rates significantly below $100 \mathrm{~Hz}$ due to the response time of the LC molecules. As we deal with static scattering systems, high adaption speeds of the wavefront are not required. A much faster alternative are deformable mirrors based on MEMS technology (micron-sized mechanical actuators), which are mainly applied in astronomy. The purpose is to correct the imaging systems for atmospheric distortions, an application termed as adaptive optics [78]. As the fluctuations in the atmosphere are fast $(>1 \mathrm{kHz})$, fast deformable mirrors are the technology of choice. However, MEMS SLMs have a lower number of individual segments $\left(\sim 10^{3}\right)$ and higher purchase costs (more than $50 \mathrm{k} €$ ).

In this section we discussed the available experimental tools to modulate a light field in a wavefront shaping experiment before the light is projected onto the scattering sample. In the following, we describe the light scattering by the random medium.

\subsection{Transmission of ultrashort pulses through ran- dom media}

In this section, we will derive the expressions to describe random transmission of a wave field through a disordered medium for illumination by ultra-short laser pulses. The description is split in two parts: At first, we describe the transport through the medium averaged over randomness in the system. In the second part we derive the expressions for the statistics of the random transmission and discuss the expressions in view of wavefront shaping experiments. 


\subsubsection{From waves to the diffusion approximation}

\section{The wave equation}

The propagation of electromagnetic waves in a random medium with the dielectric function $\epsilon(\mathbf{r})$ is governed by the wave equation

$$
\nabla^{2} \psi(\mathbf{r}, t)-\frac{\epsilon(\mathbf{r})}{c^{2}} \frac{\partial^{2}}{\partial t^{2}} \psi(\mathbf{r}, t)=0
$$

where $c$ is the speed of light in vacuum and the electric field $\psi$ is treated as a scalar. Finding the solution to the wave equation for a given disordered medium requires first the knowledge of $\epsilon(\mathbf{r})$, and second that the Maxwell's equation can be solved for the system. Obtaining the knowledge of the structure is practically impossible for most systems of interest. The analytical solution of Maxwell's equations is limited to simple geometries. Despite the tremendous progress in the field of computational methods, the numerical solution of the Maxwell's equations is limited to small systems.

\section{The radiative transfer equation}

Several approximation can be made to obtain practical analytical solutions describing the light propagation in a random medium. The first, and most severe, approximation is to neglect the wave character of the light: Radiative transport theory assumes that there is no correlation of fields and therefore powers of the fields can be added rather than the fields themselves. Despite the assumption made, the equations derived by radiative transfer theory contain information about the correlation of the fields, for which we will show an example in Sec. 2.4.2. An extensive introduction on radiative transfer and its relation to multiple scattering theory can be found in the book by Ishimaru [9].

The fundamental quantity of the radiative transfer approach is the specific intensity, defined as the average power flux density within a unit frequency band centered at frequency $\nu$ within a unit solid angle in the direction defined by the unit vector $\hat{s}$ with $d S, d \Omega, d \nu$ and $d t$ respectively the unit area, solid angle, frequency and time,

$$
I(\mathbf{r}, \hat{s})=\left[\frac{\text { Joule }}{d S d \Omega d \nu d t}\right] .
$$

We consider a system with scatterers with an extinction cross section $\sigma_{\mathrm{e}}$ and density $n$; the extinction cross section is defined by the amount of light removed from a propagating beam by scattering or absorption [79]. Both effects can be separately quantified by the individual cross sections $\sigma_{\mathrm{s}}$ for scattering and $\sigma_{\mathrm{a}}$ for absorption, with $\sigma_{\mathrm{e}}=\sigma_{\mathrm{s}}+\sigma_{\mathrm{a}}$. An important quantity to describe the light transport in the medium is the scattering mean free path, the average path length between two scattering events. In the individual-scatterer approximation it is given by the inverse product

$$
l_{\mathrm{s}}=\left(n \sigma_{\mathrm{s}}\right)^{-1} \text {. }
$$

The stationary radiative transfer equation describes the change of the specific intensity $I(\mathbf{r}, \hat{s})$ with propagation over a distance $d s$,

$$
\frac{d I(\mathbf{r}, \hat{s})}{d s}=n \sigma_{\mathrm{e}}[-I(\mathbf{r}, \hat{s})+J(\mathbf{r}, \hat{s})]
$$


On the right hand side, the equation describes the loss of the intensity propagating in direction of $\hat{s}$ due to extinction by the scatterers by the term $-n \sigma_{\mathrm{e}} I(\mathbf{r}, \hat{s})$; the gain term $n \sigma_{\mathrm{e}} J(\mathbf{r}, \hat{s})$ contains all scattering of intensity from all other directions into the direction of $\hat{s}$. However, for most systems of interest, the radiative transfer equation can only be solved numerically. To obtain practical analytical descriptions of our systems, we will further simplify our view of the scattering process in the next section.

From the loss term in Eq. 2.9 it readily follows that the intensity of an unidirectional beam incident of the medium at the interface $I(0, \hat{s})=I_{0} \delta\left(\hat{s}-\hat{s}_{0}\right)$ decreases by the Lambert-Beer law [10]

$$
I(\mathbf{r}, \hat{s})=I_{0} \delta\left(\hat{s}-\hat{s}_{0}\right) e^{-|\mathbf{r}| / l_{e}} .
$$

The decay is governed by the extinction mean free path $l_{\mathrm{e}}$, which becomes equivalent to the scattering mean free path $l_{\mathrm{s}}$ for negligible absorption.

\section{The diffusion equation}

In the diffusion approximation we assume the specific intensity distribution is almost isotropic. The angle-averaged diffuse intensity is calculated by

$$
I(\mathbf{r})=\frac{1}{4 \pi} \int_{4 \pi} I(\mathrm{r}, \hat{\mathrm{s}}) d \Omega .
$$

The diffuse intensity is proportional to the energy density $U(\mathbf{r})=4 \pi / v_{\mathrm{E}} I(\mathbf{r})$, where $v_{\mathrm{E}}$ denotes the energy velocity in the medium. Following from the diffusion approximation, the time-dependent diffusion equation

$$
\partial_{t} I(\mathbf{r}, t)=D \nabla^{2} I(\mathbf{r}, t)-D \kappa^{2} I(\mathbf{r}, t)+S(\mathbf{r}, t) .
$$

can be derived from the radiative transfer equation (see, e.g., [10]). D denotes the diffusion constant, $L_{\mathrm{abs}}=1 / \kappa$ is the absorption length and $S(\mathbf{r}, t)$ represents a source term. The diffusion constant depends on the energy velocity $v_{\mathrm{E}}$ and the transport mean free path $l_{\mathrm{t}}$ by

$$
D=\frac{1}{3} v_{\mathrm{E}} l_{\mathrm{t}} .
$$

The transport mean free path $l_{\mathrm{t}}$ is the average distance light has to propagate in the medium before its directionality is lost. If scattering from the individual scatterers is isotropic, the transport mean free path is equal to the scattering mean free path; otherwise, the quantities are related by

$$
l_{\mathrm{t}}=\frac{1}{1-\langle\cos \theta\rangle} l_{\mathrm{s}}
$$

$\langle\cos \theta\rangle$ being the average cosine of the scattering angle. In the case of strong forward scattering, $\langle\cos \theta\rangle$ is close to unity, and many scattering events are needed to randomize the direction of the light, resulting in a long transport mean free path $l_{\mathrm{t}}$.

For the systems studied in this thesis absorption is of minor influence, and we will drop the absorption term $\kappa$ and respective terms in most equations which follow. Regarding the source term $S(\mathbf{r}, t)$, we are interested in two types of sources. An 
isotropic emitter inside the medium can be modeled as a localized source of diffuse intensity inside the medium. Often we deal with a coherent beam which is incident on the interface of the disordered medium; for this case we can assume a diffuse light source at the depth of one transport mean free path $l_{\mathrm{t}}[80]$.

\section{Diffusion in a slab}

For many systems the solutions to the diffusion equation are well-known. For the most simple three-dimensional system, the infinite medium, the time-resolved propagator for the diffuse intensity in the absence of absorption is given by

$$
H(\mathbf{r}, t) \equiv \frac{1}{(4 \pi D t)^{3 / 2}} e^{-\mathbf{r}^{2} /(4 D t)} .
$$

A structure encountered in all experiments presented in this thesis is a slab. Described in cartesian coordinates, disordered material fills the space from $z=0$ up to the slab thickness $z=L$. On each side of the slab, i.e., for $z<0$ and $z>L$, we assume homogeneous media in which the light propagates without scattering; the system is translational invariant in $x$-direction and $y$-direction. In order to solve Eq. 2.12 for the slab we have to formulate appropriate boundary conditions, which must take account for internal reflection at the interfaces [81]. The internal reflections effectively hinder the light from exiting the slab, which can be described by an effective increase of the system size. Therefore $z_{\mathrm{e} 1}$ and $z_{\mathrm{e} 2}$, the so-called extrapolation lengths at the front and back interface, are introduced; the planes parallel to the interfaces at $-z_{\mathrm{e} 1}$ and $L+z_{\mathrm{e} 2}$ are called the trapping planes of the system[10]. For weakly absorbing media, the Dirichlet-type boundary conditions

$$
I\left(-z_{\mathrm{e} 1}, t\right)=0 \cup I\left(L+z_{\mathrm{e} 2}, t\right)=0
$$

lead to a sufficiently accurate solution. For absorbing and strongly scattering media, the more accurate mixed boundary conditions need to be applied [82]. The extrapolation length depends on the mean free path and the contrast between the refractive index of the homogeneous medium and the effective refractive index of the disordered medium [81-83]. For the slab we calculate it by [82]

$$
z_{\mathrm{e} 1,2}=\frac{2}{3} l_{t} \frac{1-\overline{R_{1,2}}}{1+\overline{R_{1,2}}}
$$

where $\overline{R_{1,2}}$ are the angle-average Fresnel-reflection coefficients. These coefficients are calculated using the effective refractive index of the medium, which in turn can be calculated by Maxwell-Garnett theory [84] for a known average composition of the medium.

For the boundary conditions in Eq. 2.16 we can construct the time-resolved intensity propagator of the slab using the method of images. We multiply reflect the intensity propagator of the infinite system (Eq. 2.15) at the trapping planes [85]. Here we derive the solution for unequal extrapolation lengths at the boundaries,

$$
H\left(z^{\prime}, z, \mathbf{r}_{\perp}, t\right)=\frac{1}{(4 \pi D t)^{3 / 2}} \times
$$




$$
\sum_{n=-\infty}^{\infty}\left\{e^{-\left(\mathbf{r}_{\perp}^{2}+\left[z^{\prime}-z-2 n\left(L+z_{e 1}+z_{e 2}\right)\right]^{2}\right) / 4 D t}-e^{-\left(\mathbf{r}_{\perp}^{2}+\left[z^{\prime}+z+2 z_{e 1}-2 n\left(L+z_{e 1}+z_{e 2}\right)\right]^{2}\right) / 4 D t}\right\} .
$$

We can now connect the intensity propagator to a source term $S(\mathbf{r}, t)$ to calculate the diffuse intensity $I(\mathbf{r}, t)$ at any point inside the medium. A closed expression for $I\left(z, z^{\prime}, \mathbf{q}_{\perp}, \Omega\right)$ can be derived in Fourier-transformed traversal coordinates $\mathbf{q}_{\perp}$ and in complex frequencies $\Omega$ [10]. We will later make use of the expression for the stationary case which is given by

$$
I\left(\mathbf{q}_{\perp}, z\right)= \begin{cases}J_{i n} \frac{\sinh \left(q_{\perp}\left[L_{\mathrm{e}}-z-z_{\mathrm{e}}\right]\right) \sinh \left(q_{\perp}\left[z_{0}+z_{\mathrm{e} 1}\right]\right)}{D q_{\perp} \sinh \left(q_{\perp} L_{\mathrm{e}}\right)} & z>z_{0} \\ J_{i n} \frac{\sinh \left(q_{\perp}\left[L_{\mathrm{e}}-z_{0}-z_{\mathrm{e} 1}\right]\right) \sinh \left(q_{\perp}\left[z+z_{\mathrm{e} 1}\right]\right)}{D q_{\perp} \sinh \left(q_{\perp} L_{\mathrm{e}}\right)} & z \leq z_{0} .\end{cases}
$$

$I\left(\mathbf{q}_{\perp}, z\right)$ is the diffuse intensity at depth $z$ for an source of diffuse intensity at depth $z_{0}, J_{\text {in }}$ is the power of the source in Watts and $L_{\mathrm{e}} \equiv L+z_{\mathrm{e} 1}+z_{\mathrm{e} 2}$.

The intensity scattered to the outside of the sample is obtained via the expression for the net outward flux at a point on the interface $\mathbf{r}_{\mathbf{s}}$ [85]

$$
J_{\hat{\mathrm{n}}}\left(\mathbf{r}_{\mathbf{s}}, t\right)=\left.\frac{4 \pi l}{3} \frac{\partial I\left(\mathbf{r}_{\mathbf{s}}, t\right)}{\partial z}\right|_{z=0} .
$$

A characteristic time for a diffusion process in a finite sized system of size $L$ in general is given by the Thouless time [86]

$$
\tau_{D} \equiv \frac{L^{2}}{D}
$$

It can been viewed as the characteristic time after which the diffusing quantity (here: diffuse intensity) starts to 'feel' the boundaries of system. For times longer than $\tau_{D}$, the probability distribution for the diffuse intensity inside the sample becomes spatially uniform.

In our case, the slab, the long-term behaviour of the transmitted flux is dominated by an exponential decay with the decay time $\tau_{d}$

$$
\tau_{d}=\frac{L_{e}^{2}}{\pi^{2} D}
$$

For a given geometry of a system, the timescales of interest to quantify the diffusion typically differ from the Thouless time by a geometrical prefactor.

With the radiative transfer and the diffusion approach we obtained solutions for the ensemble-averaged intensity of light transported through the medium. In the following section we will treat the characteristic properties of the wave field for a single realization of the medium.

\subsubsection{Speckle in space and time}

When a disordered slab is illuminated with a well-defined monochromatic laser beam, a random speckle pattern is observed in transmission. The spatial extent of a speckle spot on the back interface of a scattering medium is on the order of the wavelength

$\lambda$. The size of a speckle spot $\Delta x$ observed at a distance $z$ far away from the sample is 

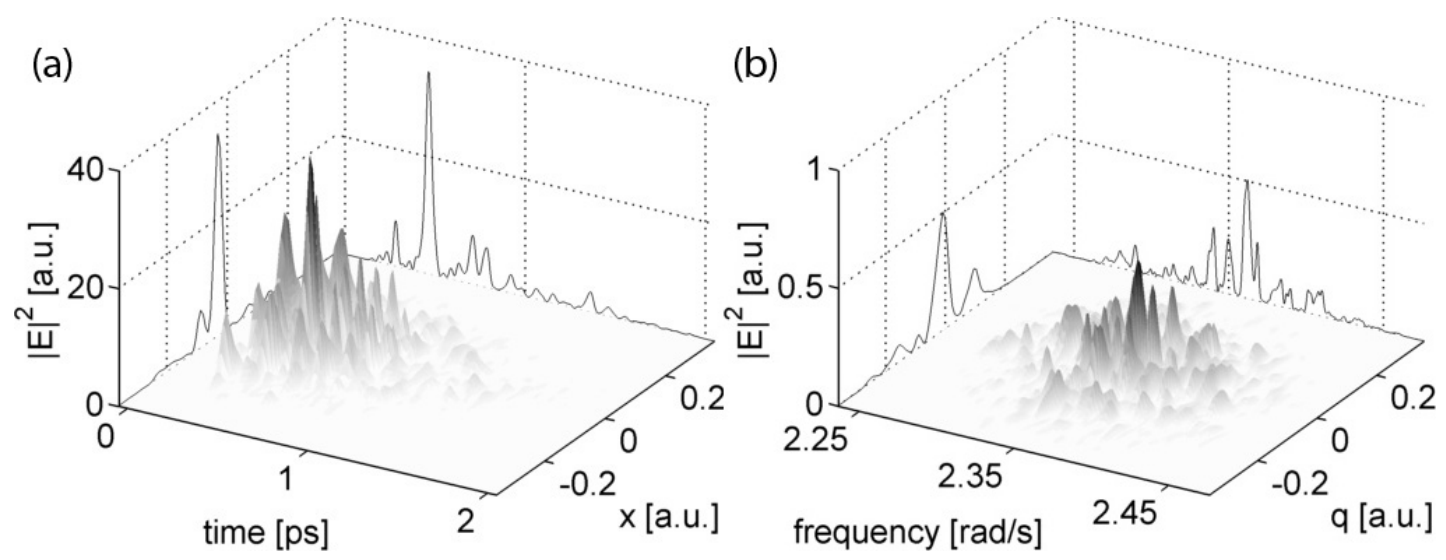

Figure 2.5: Spatiotemporal speckle field represented in the time domain and real space (a) and in Fourier space with temporal and spatial frequencies (b): The spatial distribution of the intensity changes randomly on a change of the temporal coordinate and vice versa. The plotted speckle field was generated by a simulation with the algorithm described in Sec. 4.1. The simulation models the field for pulses with initial pulse duration $\Delta t=30 \mathrm{fs}$ $\left(\lambda_{c}=800 \mathrm{~nm}\right)$ transmitted through a random slab $\left(L=15 \mu \mathrm{m}, D=58 \mathrm{~m}^{2} / 2\right)$. The spatial correlation lengths are arbitrarily chosen here, but could be readily adapted to model specific experiment parameters.

given by $\Delta x=z \lambda / d$, where $d$ is the diameter of the diffuse spot at the back interface [87].

For a light source providing short light pulses with a pulse duration shorter than the average traversal time through the medium, the speckle field in transmission is random both in space and in time (Fig. 2.5). A temporal speckle grain has the duration of roughly the pulse duration of the undistorted laser pulse. Complementary, the field has a typical grain size in the frequency domain, which, as we will see in the following, is linked to the average temporal profile of the transmission.

In the following we first introduce the formalism of the transmission matrix, which allows us to express the propagation of the wave field through the medium in terms of the transmission coefficients. Subsequently we derive the relevant expressions for the statistics and correlations of the transmission coefficients.

\section{Scattering matrix, transmission matrix and transmission coefficients}

We define a scattering channel as a propagating mode of the optical field outside the sample [15]. The scattering matrix $\mathbf{S}$ connects the ingoing and outgoing scattering channels ( $E_{\text {in }}$ and $E_{\text {out }}$ respectively) by

$$
E_{\text {in }}=\mathbf{S} E_{\text {out }}
$$

For slab or waveguide geometry as illustrated in Fig. 2.6 a clear separation into a front and back interface is given and the scattering matrix is usually written in the form

$$
E_{\text {in }}=\left(\begin{array}{cc}
r^{-+} & t^{--} \\
t^{++} & r^{+-}
\end{array}\right) E_{\text {out }}
$$




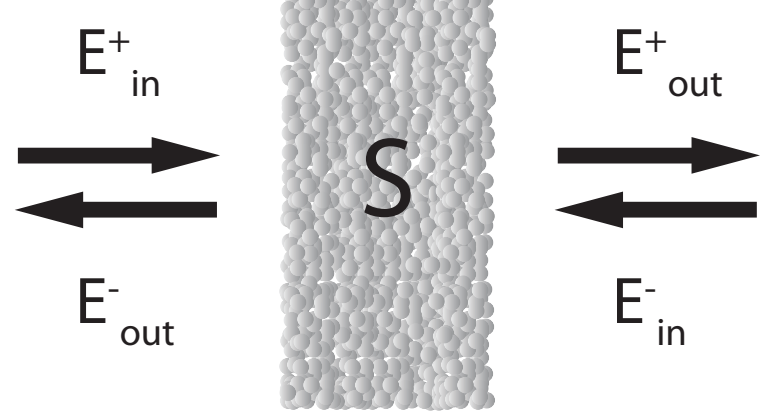

Figure 2.6: Scattering from a disordered slab.

The submatrices $t$ are called the transmission matrices, and describe the connection of the scattering channels by transmission through the sample in the direction indicated by the $(+)$ or $(-)$ sign (see Fig. 2.6). Analogously, the submatrices $r$ describe the reflection of the waves at the front interface and at the back interface.

In a transmission experiment, we are solely interested in the transmission matrix $t^{++}$, which will in the following shortly be denoted as $t$. In general, the number of elements of the transmission matrix can be restricted to the number of modes of the incident and transmitted field coupled independently to the sample. In a finitesized scattering medium, the number of supported propagating modes $N$ is finite and depends on the geometry of the system. When a wavefront is projected onto the interface of a slab sample, $N$ is given by the number of diffraction-limit spots within the area $A$ of the illuminated spot,

$$
N=C \frac{2 A}{\lambda^{2}}
$$

where $C$ is a geometrical factor on the order of one, the factor 2 accounts for orthogonal polarizations and $\lambda$ is the wavelength of the light.

We denote the complex amplitude in the ingoing channel $a$ on the left-hand side of the sample by $E_{a}$ and the complex amplitude in the outgoing channels $b$ on the right-hand side of the sample by $E_{b}$ respectively. They are connected by the complex transmission matrix elements $t_{a b}(\omega)$, which are a function of the ingoing and outgoing angle and the frequency. The total field in channel $b$ as a function of frequency is calculated by

$$
E_{b}(\omega)=\sum_{a=1}^{N} t_{b a}(\omega) E_{a}(\omega) .
$$

In some cases it is more convenient to express Eq. 2.26 in the time domain. The time-dependent transmission coefficient $t_{a b}(t)$ is the impulse response in direction $b$ to a delta-pulse incident from angle $a$,

$$
t_{a b}(t)=\mathcal{F}\left(t_{a b}(\omega)\right)
$$

where $\mathcal{F}$ denotes the Fourier transform. The electric field $E_{b}(t)$ due to an arbitrary 
excitation $E_{a}(t)$ is calculated by

$$
E_{b}(t)=\sum_{a=1}^{N} t_{a b}(t) \otimes E_{a}(t),
$$

where the fields $E(t)$ are connected to their counterparts $E(\omega)$ by Fourier transform.

Having introduced the formalism of the transmission matrix, we will now use this formalism to describe the correlations and statistics of the speckle wave field.

\section{Correlation of the transmission in space and frequency}

Although the transmission through the slab is random, the speckle pattern maintains certain correlations with respect to a change of the wavelength or the angle of incidence of the illuminating beam. We here introduce a description of these correlations in order to explain and characterize a spatiotemporal speckle pattern as it is illustrated in Fig. 2.5. Extensive and thorough reviews on correlations can be found in the literature $[88,89]$.

We denote the transmission of the intensity from the ingoing channel $a$ (with incidence angle corresponding to perpendicular momentum $q_{\perp, a}$ ) to the outgoing channel $b$ (perpendicular momentum $q_{\perp, b}$ ) by $T_{a b}=\left|t_{a b}\right|^{2}$. The correlations

$$
\begin{aligned}
C_{a b a^{\prime} b^{\prime}}(\omega, \Delta \omega) & =\frac{\left\langle T_{a b}(\omega) T_{a^{\prime} b^{\prime}}(\omega+\Delta \omega)\right\rangle-\left\langle T_{a b}(\omega)\right\rangle\left\langle T_{a^{\prime} b^{\prime}}(\omega+\Delta \omega)\right\rangle}{\left\langle T_{a b}(\omega)\right\rangle\left\langle T_{a^{\prime} b^{\prime}}(\omega+\Delta \omega)\right\rangle} \\
& =C_{a b a^{\prime} b^{\prime}}^{(1)}+C_{a b a^{\prime} b^{\prime}}^{(2)}+C_{a b a^{\prime} b^{\prime}}^{(3)}
\end{aligned}
$$

can be separated into three terms [90]: short-range $C^{(1)}$, long-range $C^{(2)}$ and infiniterange $C^{(3)}$ correlations. The term for the short-range $C^{(1)}$ correlations can be evaluated to yield [90]

$$
C_{a b a^{\prime} b^{\prime}}^{(1)}=\left\langle T_{a b}\right\rangle\left\langle T_{a^{\prime} b^{\prime}}\right\rangle \delta_{\Delta q_{a}, \Delta q_{b}} F_{1}\left(\Delta q_{a} L\right),
$$

where $\Delta q$ is the momentum difference and $F_{1}$ is a reduced correlation function, which is calculated in the following paragraph.

The correlation manifests both in frequency and spatially. On a change of frequency of the incoming beam, the correlation of the speckle pattern decays exponentially. On a gradual change of the angle of incidence of the incoming beam (at fixed frequency) the change of the transmitted speckle pattern has two aspects. Firstly, the transmitted speckle pattern is translated along with the incident beam, which is called the memory effect [91]. Secondly, the correlation of this shifting pattern with the original pattern again decays exponentially, which is the $C^{(1)}$ correlation. Therefore the $C^{(1)}$ correlation is only non-zero when the measurement 'compensates' for the memory effect, which means that the change of perpendicular momentum of the incident beam and the detection direction are equal, expressed by the $\delta$ in Eq. 2.31. The name of the long-range $C^{(2)}$ correlations indicates that they are maintained for scattering channels far apart. The infinite-range $C^{(3)}$-correlation is the analog to the universal conductance fluctuations in electronic systems. The contribution of $C^{(2)}$ is on the order of $g^{-1}$ and the contribution of $C^{(3)}$ on the order of $g^{-2}$, where $g$ is the conductance defined as $g=\sum_{a b} T_{a b} \cdot C^{(2)}$ and $C^{(3)}$ correlations are negligible for our samples, since we can assume $g \gg 1$. 
An expression for the reduced correlation function $F_{1}$ in the $C^{(1)}$ correlation function can be derived via the correlation function $C_{E}$ of the electric field $E_{a b}=E_{0} t_{a b}$ $[89,92]$

$$
C_{E}=\frac{\left\langle E_{a b} E_{a^{\prime} b^{\prime}}^{*}\right\rangle}{\left\langle\left|E_{a b}\right|\right\rangle\left\langle\left|E_{a^{\prime} b^{\prime}}\right|\right\rangle}
$$

by

$$
C_{a b a^{\prime} b^{\prime}}^{(1)}=\left|C_{E}\right|^{2}
$$

In a thick disordered slab with negligible absorption the $C^{1}$ - term is given by [93]

$$
C_{a b a^{\prime} b^{\prime}}^{(1)}(\Delta \omega)=\left|\frac{M L}{\sinh (M L)}\right|^{2}
$$

where $M^{2}=\Delta q_{\perp}^{2}+\frac{i \Delta \omega}{D} . \Delta q_{\perp}^{2}$ is the perpendicular momentum difference and $L$ the sample thickness. The half-width at half maximum of $C^{(1)}$ in the form of Eq. 2.34 is the correlation frequency

$$
\Delta \omega_{1}=2.92 \pi \frac{D}{L^{2}} \approx \tau_{d}^{-1}
$$

which differs by less than $10 \%$ from the inverse decay time $\tau_{d}^{-1}$ obtained from the solution of the diffusion equation for the random slab. In general, for thin or absorbing samples the extrapolation length $z_{0}$ and the absorption coefficient $\kappa$ need to be accounted for. The expression for $C^{(1)}$ in this general case is slightly more complex and can be found in [10,94].

\section{Statistics of amplitude, phase and phase delay time}

The expressions above describe correlations of the transmitted intensity. In this section we describe the statistics of the electric field, in particular the phase. The theory presented in the following has been worked out by van Tiggelen et al. [95] and agrees well with microwave experiments by the same group [96] and with optical measurements by Johnson et al. [97].

For our scattering samples we can assume Gaussian statistics of the transmitted field. This Gaussian assumption is valid when a high number of independent paths contributes to the field at the back surface of the disordered slab. The Gaussian assumption is equivalent to the $C^{(1)}$-approximation made in the previous section based on a conductance $g \gg 1$. The central limit theorem predicts that in this situation the real and imaginary parts of the transmission coefficients $t_{a b}$ are respectively described by a normal distribution. Equivalent is the description in terms of a circular Gaussian process [98], for which the field amplitude $\left|t_{a b}\right|$ is Rayleigh-distributed and the phase $\phi=\arg \left(t_{a b}\right)$ is uniformly distributed between 0 and $2 \pi$.

From the probability distribution of the transmission coefficients alone, we cannot deduce information about the dynamics of the diffusion process. We are interested in the group delay time $\phi^{\prime}=d \phi / d \omega$, which is the induced time delay for a narrow-band wave package. The probability distribution is given by

$$
P\left(\widetilde{\phi^{\prime}}\right)=\frac{Q}{2\left[\left(\widetilde{\phi^{\prime}}-1\right)^{2}+Q\right]^{\frac{3}{2}}},
$$


where $\widetilde{\phi^{\prime}}=\phi^{\prime} /\left\langle\phi^{\prime}\right\rangle$ and $Q$ is a dimensionless parameter which can be calculated from the Taylor expansion of the field correlation function $C_{E}(\Delta \omega)=1+i \tau_{t}(\Delta \omega)-b(\Delta \omega)^{2}+$ $\mathcal{O}(\Delta \omega)^{3}$ (see Eq. 2.32). The characteristic traversal time for the diffuse transmission (the average time the light takes to propagate through the sample [99]) is $\tau_{t}=\left\langle\phi^{\prime}\right\rangle$ and $Q=2 b / \tau_{t}^{2}-1$. The probability distribution $P\left(\widetilde{\phi^{\prime}}\right)$ describes the occurrence of a certain value phase delay time, but does not account for the transmission $T$ connected to this delay time. Therefore, the weighted phase delay time

$$
W=T \phi^{\prime} .
$$

is usually more insightful. Its probability distribution can be calculated to

$$
P(\widetilde{W})=\frac{1}{\sqrt{1+Q}} \exp \left(\frac{-2|\widetilde{W}|}{\operatorname{sgn} \widetilde{W}+\sqrt{1+Q}}\right) .
$$

\section{Number of independent spatial and spectral scattering channels}

In the two preceding sections we derived the expressions for the spatial and temporal correlations of the scattered wave field. Here we interpret the correlations in terms of a wavefront shaping experiment: In a wavefront shaping experiment the waves in the incident channels are modified to control the field behind the medium. The question which arises is how many independent degrees of freedom contribute to a focal point of the field at a point behind a thick scattering medium. Here, we examine the situation for a single point at the back interface of a disordered slab, which is illuminated from the front side. We can distinguish between independent spatial and spectral scattering channels [100, 101].

Spatially, only light entering the medium within a certain area opposite of the focal point will contribute to the field at the considered point. The diameter (FWHM) of this area scales linearly with the slab thickness $L$ with a proportionality factor on the order of one. The profile can be calculated exactly by Eq. 2.19 for given scattering properties of the medium. The number of independent spatial channels is given by the diffraction limited spots in this area,

$$
N_{s}=C \frac{L^{2}}{\lambda^{2}},
$$

where $C$ is a prefactor on the order of one. Analog to Eq. 2.25 we can add an additional prefactor 2 when both polarizations of the incident field are controlled independently.

The number of spectral channels per bandwidth of the incident light is given by the $C^{(1)}$-correlation frequency (Eq. 2.35). Complementary, we can interpret this number as the number of temporal channels in a time-domain picture: A pulse of a bandwidth $\Delta \omega$ narrower than the frequency $\Delta \omega_{1}$, or a pulse width the temporal duration $\Delta t$ larger than the decay time $\tau_{d}$ will not be significantly distorted spectrally or temporally. With Eq. 2.22 and respectively Eq. 2.35 the expression for the number of temporal or spectral channels is

$$
N_{t}=\frac{\Delta \omega}{\Delta \omega_{1}} \approx \frac{\tau_{d}}{\Delta t} \approx \frac{\Delta \omega_{0}}{D \pi^{2}} L^{2}
$$


(a)

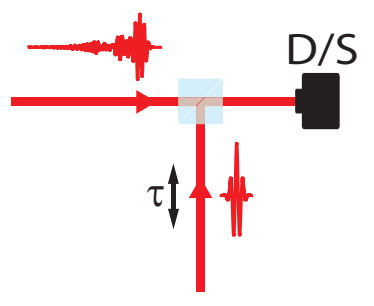

(b)

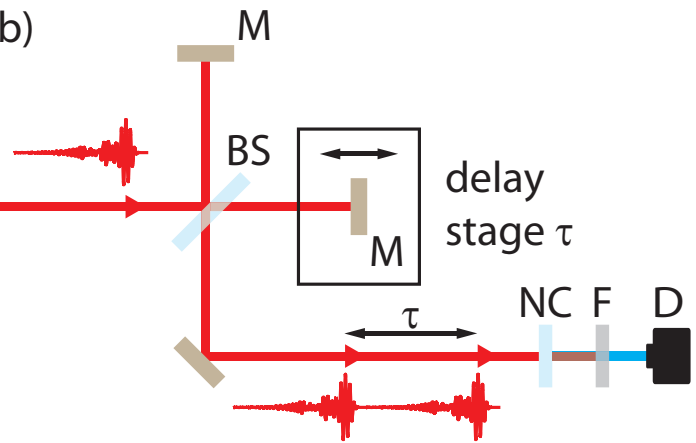

Figure 2.7: Detecting ultra-short speckle pulses by spectral or temporal interferometry (a) or by interferometric autocorrelation (b). D: photodetector; S: spectrometer; M: mirror; BS: beam splitter; NC: nonlinear-crystal; F: filter.

We see that both the number of spectral and temporal channels scale with the square of the dimension of the medium.

In this section we described the light transport through the random medium and the typical properties of speckle pulses scattered from a random sample. In the following, we discuss the experimental schemes for detecting these speckle pulses.

\subsection{Ultrashort pulses - detection}

The best time resolution which can be achieved by photodetectors is limited to about one picosecond, which is one to two orders of magnitude slower than typical pulse durations and two to three orders slower than an optical cycle in the visible range. In order to bypass the limitation set to the direct detection, numerous types of characterization techniques for ultra-short laser pulses have been developed in the last three decades. The techniques can be classified in linear and non-linear, cross-referenced and self-referencing, and complete and incomplete. Linear techniques have a higher sensitivity than non-linear techniques, but are not always practical. Cross-referencing techniques are usually more accurate than self-referencing techniques, but require a known reference signal for the characterization of the unknown pulse. Some techniques are incomplete and allow only partial extraction of information about the pulse, but they are usually experimentally easier to implement than complete characterization techniques. For a broad introduction on the topic we refer to the general literature $[62-64,102]$. In this section we single out the two main techniques which we apply in our experiments to detect ultra-short speckle pulses.

\subsubsection{Spectral and temporal interferometry}

We have sketched the detection scheme of temporal and spectral interferometry in Fig. 2.7a [103]. In the scheme of the classifications of methods made above, temporal interferometry is linear, cross-referenced and complete. The unknown pulse is overlapped with a known reference pulse on a beam splitter. A photodetector records the time-integrated interference signal as a function of the delay time $\tau$ of the reference pulse, 


$$
S(\tau)=\int_{-\infty}^{+\infty}\left|E_{\mathrm{sig}}(t)\right|^{2} d t+\int_{-\infty}^{+\infty}\left|E_{\mathrm{ref}}(t)\right|^{2} d t+\int_{-\infty}^{+\infty} E_{\mathrm{ref}}^{*}(t-\tau) E_{\mathrm{sig}}(t) d t+c . c .
$$

Fourier transformation of $S(\tau)$ yields the product $E_{\text {ref }}^{*}(\omega) E_{\text {sig }}(\omega)$, from which we can extract $E_{\text {sig }}(\omega)$ providing that the reference signal is well-characterized. Experimentally it is often more convenient to keep the time delay $\tau$ fixed and replace the detector by a spectrometer which then records

$$
S(\omega ; \tau)=\left|E_{\mathrm{sig}}(\omega)\right|^{2}+\left|E_{\mathrm{ref}}(\omega)\right|^{2}+\left|E_{\mathrm{sig}}(\omega)\right|\left|E_{\mathrm{ref}}(\omega)\right| \cos \left[\omega \tau+\phi_{\mathrm{sig}}(\omega)-\phi_{\mathrm{sig}}(\omega)\right] .
$$

From the Fourier-transform of $S(\omega)$, the cross-correlation in the time-domain is extracted, which is transformed back to the frequency domain to obtain $E_{\text {ref }}^{*}(\omega) E_{\text {sig }}(\omega)$. The approach was first applied to speckle characterization by Johnson et al. [97].

In the experiment described in chapter 3 we employ temporal interferometry, because it can be readily combined with an experimentally convenient implementation of heterodyne detection [104]. We describe the experimental realization in Sec. 3.A.1. For our purposes, heterodyne detection brings two advantages. Firstly, it provides a high signal-to-noise ratio [104], which is crucial for the detection of weak speckle pulses. Secondly, the recorded signal from the photodetector can be assumed proportional to the amplitude of the electric field $E_{\text {sig }}(\tau)$. Therefore the signal can be directly used as a feedback for wave-front shaping optimization without any further processing (see chapter 3).

Temporal and spectral interferometry as depicted in Fig. 2.7 are in principle singlemode detection techniques and we will use them as such. One approach to fully characterizing a spatiotemporal speckle field spatially and temporally is to spatially scan the mode which is picked up and interfered with the reference signal. Scanning the point of illumination and detection on a nanostructure can be used for spectral interference microscopy [105]. A technically different approach is to use a spectrometer with a 2D detector array (e.g., a CCD camera) and to record the spectrum as a function of one spatial coordinate [106].

\subsubsection{Autocorrelation}

Autocorrelators are the standard tool used to monitor the output of a short-pulse laser system [107, 108]. A variation of the technique allows us to characterize speckle pulses inside a random medium, as we show in chapter 6 .

We have schematically drawn a standard realization in a collinear alignment in Fig. 2.7b. As the name indicates, the autocorrelation is a self-referencing technique. The pulse which is to be examined is overlapped with a time-shifted replica on a nonlinear crystal (NC) in which the second (or higher) harmonic of the light is generated. The harmonic signal is detected with a slow detector as a function of time-delay. The detection can be performed by averaging over optical cycles (intensity autocorrelation) or by resolving the each optical cycle of the interfering pulses (interferometric autocorrelation). 
A disadvantage of the technique is that neither the intensity autocorrelation nor the interferometric autocorrelation allow the full retrieval of the electric field $E_{\mathrm{sig}}(t)$ [63]. However, with prior knowledge about the spectrum or the pulse shape, the pulse duration of laser pulses can be estimated in many cases. For speckle pulses we know that they consist of mutually incoherent sub-pulses of a duration on the order of the band-width limit. From the autocorrelation of speckle pulses we can obtain the average duration of the sub-pulses and the overall distribution of the diffuse intensity.

A great advantage of the autocorrelation is the fact that it is self-referencing, such that no reference pulse is required at the location of the nonlinear crystal. Furthermore, the splitting of the pulse into two copies is a linear operation. Therefore we can place the dispersive element (the random medium) either before or behind the Michelson-type interferometer in Fig. 2.7b [109]. Going one step further, the crystal can be placed directly inside the scattering medium. With this approach, we demonstrate that we are able to measure the dispersion of an ultra-short laser pulse in a small volume inside a random material (see chapter 6).

\subsection{Analogies and differences between optics, mi- crowaves and ultrasound experiments}

Conceptually the wavefront shaping scheme in Fig. 2.1 can be implemented with all types of waves. For certain proof-of-principle experiments, electromagnetic waves in the microwave regime and ultrasound are appealing since the required experimental apparatus is usually easier to handle than in the optical regime.

Ultrasound covers a frequency range form $100 \mathrm{kHz}$ to $50 \mathrm{MHz}$. In water the wavelength at $1 \mathrm{MHz}$ is $1.5 \mathrm{~mm}$. Compared to optics, the generation and detection of signals is far simpler: piezo-electric ultrasound transducers act both as receivers and emitters. The transducers can emit and receive signals over a large bandwidth, typically reaching a relative bandwidth of $100 \%$, which by far exceeds the bandwidth of optical systems. In this fashion they can be conveniently employed for time reversal experiments [39]. In chapter 7 we make use of this advantage in ultrasound: We measure the broadband transmission matrix of a random medium, by scanning a emitter-receiver pair on both sides of the medium. On the other hand, due to practical constraints of size and required electronics, the maximum number of individual transducers is limited to about $10^{2}$ spatial elements, which is much lower than the number of degrees of freedom provided by a SLM in optics. The microwave regime comes with similar advantages and disadvantages compared to optics. Microwave antenna can emit radiation in the range from $1 \mathrm{GHz}$ to $100 \mathrm{GHz}$, corresponding to a free space wavelength from $3 \mathrm{~mm}$ to $30 \mathrm{~cm}$. Broadband signals over a large bandwidth can be synthesized, and typical microwave antennas works equivalently as receiver and emitter. Similar to acoustics, the number of spatial degrees of freedom are limited for practical reasons, due to the scaling of the antenna system and the connected electronics. 


\subsection{Summary}

In this chapter, we presented and described the elements of a short-pulse wavefront shaping experiment. First we provided a description for ultrashort light pulses. Then we described the working principle of spatial light modulator applied to ultrashort pulses and evaluated the options to shape short-pulse laser beams spatially, temporally and spatiotemporally. In the experiments presented in chapters 3,5 and 6 , the described spatial light modulation scheme will be used. Thereupon we gave an analytical description of the propagation of ultrashort laser pulses through multiple scattering media. This description provides the basis for simulation in chapter 4 and the modeling for all experimental chapters. At last we describe the two schemes for the time-resolved detection of ultrashort laser pulses which are applied in the experiments: We introduced temporal interferometry, which will be used in chapter 3 and we illustrated the principle of the short-pulse autocorrelation, which is applied the experiments in chapters 5 and 6 . 



\section{CHAPTER}

\section{Control of light transmission through opaque scattering media in space and time}

We report the experimental demonstration of combined spatial and temporal control of light transmission through opaque media. This control is achieved by solely manipulating spatial degrees of freedom of the incident wavefront. As an application, we demonstrate that the present approach is capable to form bandwidth-limited ultrashort pulses from the otherwise randomly transmitted light with a controllable interaction time of the pulses with the medium. Our approach provides a new tool for fundamental studies of light propagation in complex media and has potential for applications for coherent control, sensing and imaging in nano- and biophotonics.

Concentrating light in time and space is critical for many applications of laser light. Broadband mode-locked lasers provide the required ultrashort light pulses for multiphoton imaging [110, 111], nanosurgery [53], microstructuring [112], ultrafast spectroscopy $[113,114]$ and coherent control of molecular dynamics or of nanooptical fields $[50,51,76]$. Multiple random scattering in complex media severely limits the performance of these methods, but often is an unavoidable nuisance in many systems of interest, such as biological tissue or nanophotonic structures [115]. Spatially, random scattering strongly distorts a propagating wavefront, creating the well-known speckle interference pattern [116]. In the time domain, ultrashort pulses are strongly distorted and widely stretched due to the broad path length distribution in multiple scattering media [117]. These temporal and spatial distortions are not separable [100].

The content of this chapter has been published as: J. Aulbach, B. Gjonaj, P. Johnson, A. P. Mosk, and A. Lagendijk, Phys. Rev. Lett. 106103901 (2011). 
There is a strong interest in improving applications of ultrashort laser pulses in complex scattering media. Phase conjugation has been applied to spatially focus light from a short-pulse laser source through a thin scattering layer [27]. Similarly, phase conjugation is applied to correct distortions of the ballistic wavefront to improve the resolution of two photon microscopy [118]. Coherent control of two-photon excitation through scattering biological tissue has been demonstrated [119]. Those experiments share the common limitation that the control is limited only to those photons that take the shortest paths through the disordered media and arrive at the target volume without being multiply scattered.

Recently it was demonstrated that random scattering can actually be beneficial rather than detrimental for the performance of optical systems. Applying a shaped wavefront of monochromatic light to a strongly scattering medium, Vellekoop et al. achieved spatially controlled focusing in transmission [13] and on fluorescent molecules inside the medium [14]. These findings have opened new possibilities for imaging in optically thick biological matter [120] and allow trapping particles through turbid media [121]. All of these studies used monochromatic light sources, and therefore only allowed spatial control over the scattered light. Related techniques which allow coherent focusing in scattering media are known from ultrasound [40] and microwaves [42]. The frequency of those types of waves is low enough that electronic transducers or microwave antennas can be used to time reverse waves, which redirects the waves towards their source. This technique has successfully helped to improve imaging resolution [39] and communication bandwidth [46, 122].

In this chapter, we generalize the concept of wavefront shaping to the regime of broadband light. We report the first experimental demonstration of combined spatial and temporal control of light transmission through random scattering media. By only controlling spatial degrees on freedom of the incident wave, we control the field amplitude at a selected point in space and time behind the sample. This enables us to create an ultrashort pulse from the otherwise randomly transmitted light. We can control the amount of time the optimized pulse stays in the sample and thereby select the path length of the light through the medium.

In Fig.3.1 we show a simplified scheme of our experimental realization. Pulses

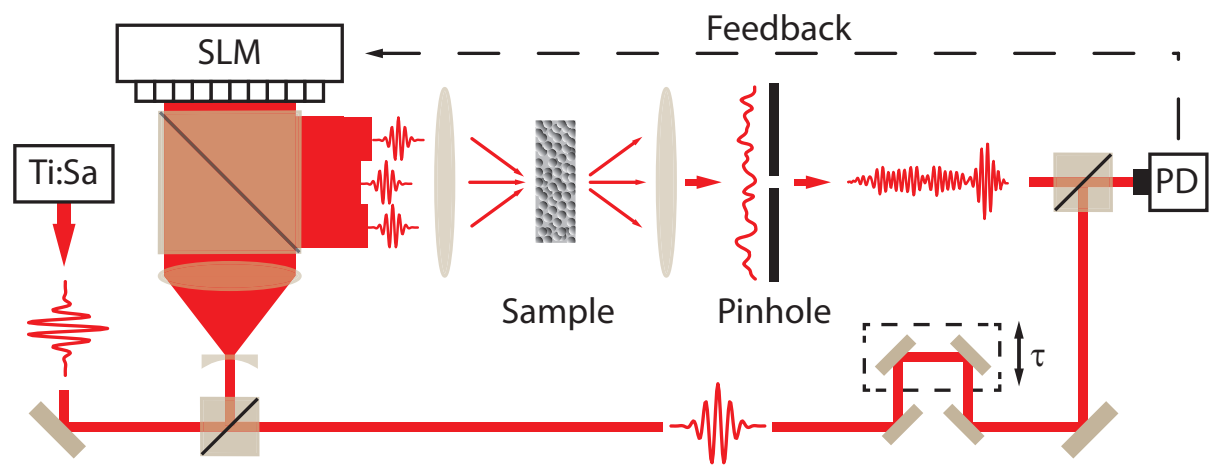

Figure 3.1: Experimental setup (see text).

from a Ti:Sapphire laser (duration $64 \mathrm{fs}$, center wavelength $795 \mathrm{~nm}$ ) illuminate a twodimensional phase-only spatial light modulator (SLM). The SLM pixels are grouped into $\mathrm{N}$ independent segments each of which induces a controllable phase shift $\Delta \Phi_{i}$. 
The light is subsequently focused onto a layer of strongly scattering titanium dioxide pigment (thickness $\mathrm{L}=13.5 \mu \mathrm{m}$, transport mean free path: $l_{t}=1 \mu \mathrm{m}$ [123]). The transmitted light appears as a spatiotemporal speckle pattern. In the experiment, we optimize the field amplitude at a selected point in space and time. A pinhole fixes the spatial coordinate. To fix the time, the speckle pulse is overlapped with a reference pulse in a heterodyne detection scheme in a configuration similar to [124]. For an extended technical and analytical description of this technique, we refer the reader to appendix 3.A.1. The heterodyne signal exactly corresponds to the cross-correlation of the speckle pulse and the reference pulse. Effectively, it is an instantaneous measurement of the transmitted field amplitude at the delay time of the reference pulse $\tau$. This signal serves as a feedback for an optimization algorithm, which controls the incident wavefront via the SLM.

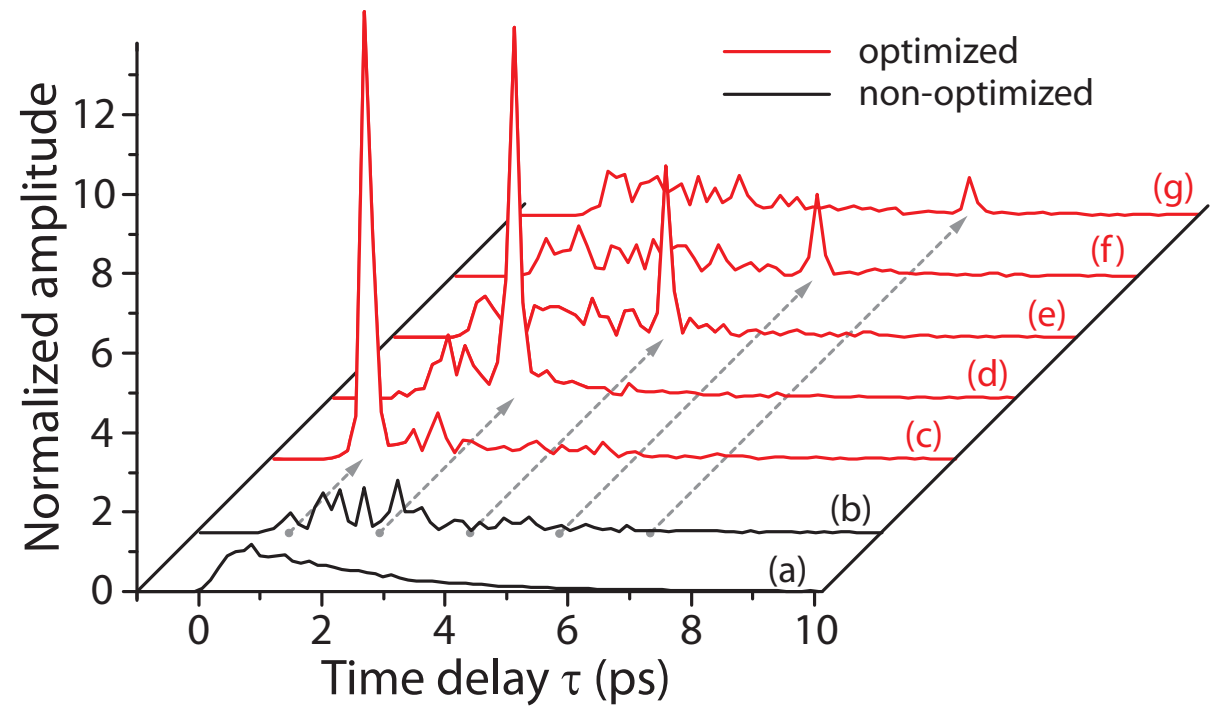

Figure 3.2: Optimized and random speckle pulses. (a) Amplitude of heterodyne signal of a non-optimized pulse as a function of time delay, averaged over 50 random speckle pulses. (b) Typical single random speckle pulse. (c)-(g) Amplitudes of single pulses after optimization at different time delays which are indicated by the dashed arrows. The optimization has been performed by dividing the SLM into 300 segments. The optimization generates strong, short pulses from diffuse light. The zero delay position is at the maximum amplitude with no sample. The plotted curves have been normalized to the maximum of the average nonoptimized heterodyne signal (factor $1.53 \mathrm{mV}^{-1}$ ).

The principle of the experiment can be described as follows. Light reflected from a single segment on the SLM is transmitted through the sample, giving rise to the field $E_{i}(t)$ at the detector. Its phase can be modified by a time-independent phase shift $\Delta \Phi_{i}$ via the SLM. The total field scattered into the detector $E_{\text {out }}(t)$ is therefore given by the sum over all segments

$$
E_{\text {out }}(t)=\sum_{i=1}^{N} E_{i}(t) e^{i \Delta \Phi_{i}} .
$$

Multiple scattering allows us to assume that the contributions $E_{i}(t)$ from the 


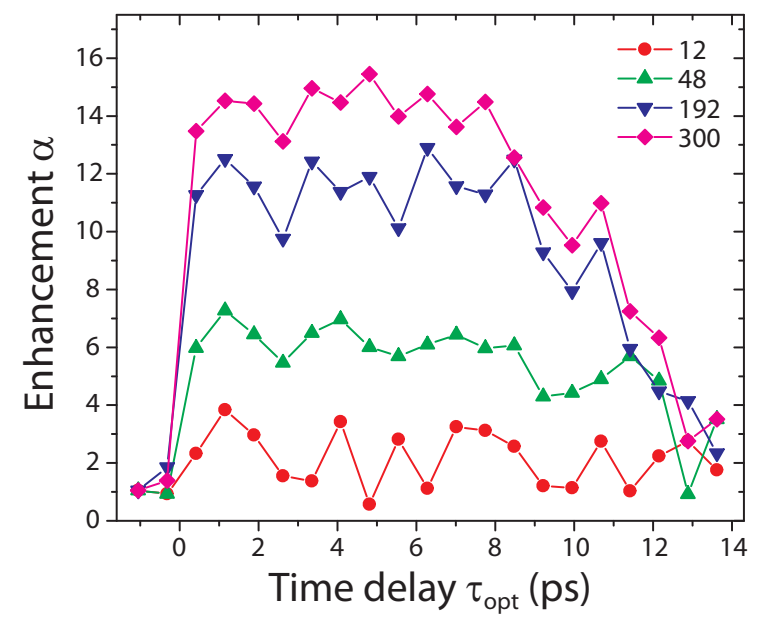

Figure 3.3: Enhancement $\alpha$ versus selected time delay $\tau_{\text {opt }}$ for different number of segments $\mathrm{N}$ on the spatial light modulator.

different segments at every single point in time $t$ are uncorrelated random variables with Rayleigh distributed amplitudes $\left|E_{i}(t)\right|$ and uniformly distributed phases $\Phi_{i}(t)$ [95]. For the non-optimized case, the resulting field $E_{\text {out }}(t)$ can be viewed as the result of a random walk in the complex field plane. After the optimization, all contributions are in phase, adding up constructively. The average amplitude enhancement is given by [13]

$$
\begin{aligned}
\langle\alpha\rangle & =\frac{\left\langle\left|E_{\mathrm{opt}}\right|\right\rangle_{\mathrm{rms}}}{\left\langle\left|E_{\mathrm{rnd}}\right|\right\rangle_{\mathrm{rms}}} \\
& =\left(\frac{\pi}{4}(N-1)+1\right)^{1 / 2} \approx\left(\frac{\pi}{4} N\right)^{1 / 2} .
\end{aligned}
$$

The derivation of this expression is included in the appendix 3.A.4. Since the instantaneous field amplitude is optimized, the instantaneous intensity accordingly increases, with an average intensity enhancement $\eta=\alpha^{2}$. The simple model leading to Eq. 3.2 models the amplitude enhancement at one point in time, but does not provide a prediction for the resulting pulse duration, i.e., the interval over which the amplitude is enhanced. We address this point later in this chapter.

The non-optimized data was obtained by setting random phase values to the SLM segments. The optimization algorithm adjusts the phase shifts $\Delta \Phi_{i}$ such that the amplitude of the heterodyne signal is maximized. We performed the optimization at 20 equidistant time delays $\tau_{\text {opt }}$ between -1.05 ps to +13.6 ps. For each $\tau_{\text {opt }}$, the optimization was performed four times, with $\mathrm{N}=12,48,192$ and 300 segments, respectively, each time starting from a new random phase pattern.

Our main result is displayed in Fig. 3.2, showing the amplitudes of both the non-optimized (black lines) and the optimized (red lines) pulses for different time delays $\tau_{\text {opt }}$ and $\mathrm{N}=300$ segments on the SLM. The long time-tail of the average nonoptimized transmission reflects the broad path length distribution which has been observed in similar earlier studies [117]. The optimized amplitudes show sharp, distinct peaks with dramatically increased amplitudes at the desired time delay. We can control the amount of time the optimized pulses stay in the sample by the time 
delay $\tau_{\text {opt }}$, and by that we control the path length of the pulses through the sample. Note that the heterodyne signal is proportional to the field amplitude, the intensities exhibit even more pronounced optimized peaks.

The enhancement $\alpha$ versus time delay $\tau_{\text {opt }}$ is shown in Fig. 3.3. Its magnitude, depending on the number of segments on the SLM, is constant from zero to several picoseconds time delay. This result shows that our method works for short light paths as well as for light paths more than ten times longer than the sample thickness. For long time delays a continuous decrease of $\alpha$ is observed, which is related to the noise level of the experiment. We include a quantitative analysis of this effect in appendix 3.A.3.

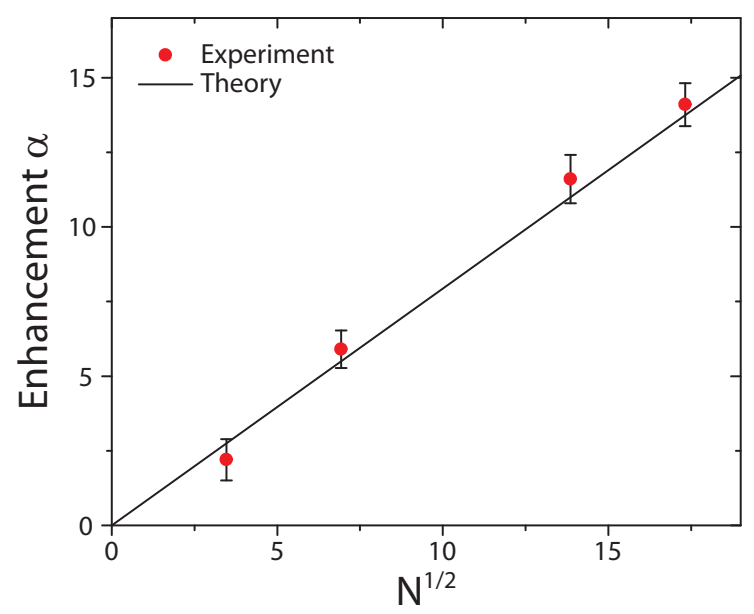

Figure 3.4: Average amplitude enhancement $\alpha$ (red dots) as a function of the square root of the number of segments $N$. The solid line is given by the expected $\alpha=0.90\left(\frac{\pi}{4} N\right)^{1 / 2}$, without any free parameter.

Figure 3.4 shows the average enhancement in the constant regime in Fig. 3.3 versus $N$ together with the enhancement expected from theory (Eq. 3.2), $\langle\alpha\rangle=\sigma\left(\frac{\pi}{4} N\right)^{1 / 2}$. The prefactor $\sigma=0.90$ corrects for the non-uniform illumination of the SLM surface with a truncated Gaussian beam, which effectively leads to a reduction of the number of used segments (see appendix 3.A.4). Our model matches the data well with no adjustable parameters.

The measurement of the output pulse duration reveals an interesting characteristic of the optimization method. Independent of the time delay, the optimized pulses have an (intensity) duration of $\Delta \mathrm{t}_{\mathrm{opt}}=115 \mathrm{fs}$, calculated from the average width of the cross-correlation peaks (Fig. 5). The optimized pulses are lengthened compared to the input pulses $\left(\Delta \mathrm{t}_{\text {in }}=64 \mathrm{fs}\right)$. What follows is a qualitative explanation of this effect. Further details are included in appendix 3.A.2. The increased duration corresponds to spectral narrowing due to the optimization procedure. Transmission through the random medium introduces strong random phase and amplitude fluctuations within the laser bandwidth (frequency speckle) [95]. This means that a single segment of the phase plate, which adds an almost frequency-independent phase shift, cannot optimize all frequencies equally well. The optimization is biased towards frequencies of higher amplitude, since they contribute higher to the feedback signal. Averaged over many SLM segments, these are the frequencies in the center of the 


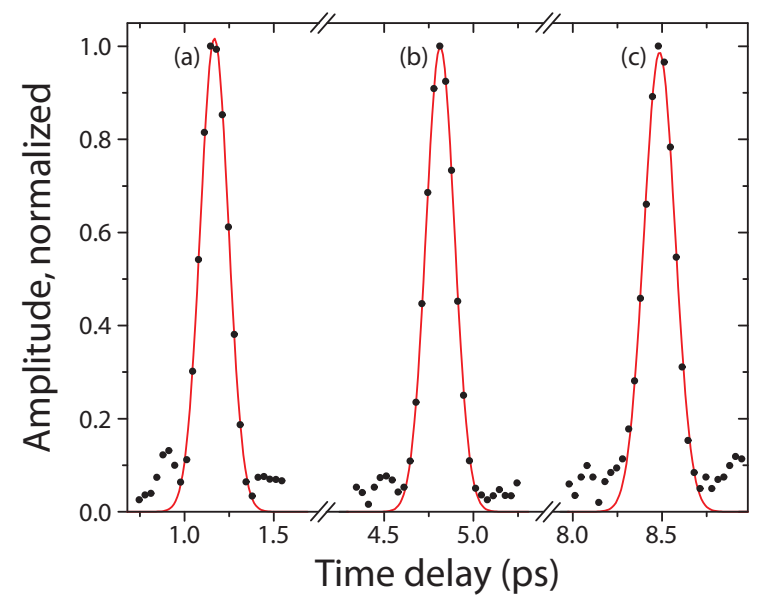

Figure 3.5: Detailed cross-correlation scans after optimization with $\mathrm{N}=300$ segments around the time delays $\tau_{\text {opt }}=1.1 \mathrm{ps}(\mathrm{a}), 4.8 \mathrm{ps}$ (b) and $8.5 \mathrm{ps}(\mathrm{c})$, together with a Gaussian fit (red lines). The width (FWHM) of the peaks shows no significant dependence on the time delay, with an average of $\Delta \tau_{\mathrm{opt}}=(190 \pm 7) \mathrm{fs}$.

Gaussian spectrum of the laser, resulting in a narrowing of the resulting spectrum. We investigated the dependence of this effect on the number of segments $N$ by a numerical simulation, which generates random spectra based on the parameters of our experiment. These spectra were Fourier-transformed and their sum optimized in time to mimic our experimental optimization procedure. For a low number of segments, the spectral amplitude and phase is dominated by the randomness from the scattering. For an increasing number of segments, the optimized pulses exhibit an increasingly smooth Gaussian amplitude and a flat spectral phase, with a bandwidth narrower than the input spectrum. The resulting pulse duration converges to $115 \mathrm{fs}$, in perfect agreement with our experiments. The method is capable of creating bandwidth-limited pulses, but since it is based on linear interferometry, the optimization can be equally applied to adapt other pulse shapes or likewise to compensate material dispersion present in the optical path.

The time-integrated intensity (energy) of the pulse with the highest peak depicted in Fig. 3.2 is 13.5 times higher than the energy of the average non-optimized pulse. In addition to the temporal optimization, overall more light is transmitted into the detected channel, demonstrating that the scattered light is controlled spatially and temporally. A SLM alone, without a random scattering medium, offers only spatial control, while frequency domain pulse shaping techniques [59] provide temporal control only. Our method exploits the mixing of spatial and temporal degrees of freedom by the random medium [100], to control the transmitted light in two spatial and one temporal dimension by only controlling spatial degrees of freedom on the two-dimensional SLM. On the one hand, the conversion of spatial degrees of freedom into temporal ones comes to the price of a speckle background, which on the other hand is easily outweighed by the enormous number of degrees of freedom provided by state-of-the-art SLMs. The large number of controllable spatial degrees of freedom is a great advantage over frequency domain pulse shaping techniques. Spatiotemporal control of the light field allows a far more generalized application of present coherent control schemes and marks a further step towards optical time reversal. 
In the experimental realization presented here, we optimized the pulse front using linear interferometry as feedback signal. The optimization of a non-linear response, like second-harmonic generation, will also lead to a comparably optimized pulse [125]. Using second-harmonic emission from nanoparticles [27] or two-photon fluorescence from dyes such as fluorescent proteins [111] would enable focusing ultra-short pulses inside complex media such as biological tissue, in which the propagation of nearinfrared light is dominated by multiple scattering [120]. Given the high signal to background ratio and the enhancement of energy delivered to the selected speckle spot, we envision that our method can improve approaches for selective cell destruction in tissue [126]. In view of its potential for sharp focusing, it opens interesting perspectives for nanofabrication, nanosurgery and other micromanipulation techniques.

Up to now we have not discussed the spatial extent of the optimized pulse. We use a pinhole to select a single speckle spot in the Fourier plane of the sample for optimization. We know that transmitted fields in adjacent speckle spots are uncorrelated [127], from which we can conclude that the optimization is indeed limited to the selected area (see appendix 3.A.6). An important future direction would be to investigate the spatial extent of the optimized pulse as a function of delay time [128]. A combination with spatial scanning allows the measurement of the transmission matrix of the medium [18] in one temporal and two spatial dimensions. For Anderson-localizing samples [129], the size of the optimized speckle should be strongly time-dependent [130].

In conclusion, we have experimentally demonstrated that spatial wave front shaping of a pulse front incident on a strongly scattering sample gives spatial and temporal control over the scattered light. Our approach provides a new tool for fundamental studies of light propagation and has potential for applications in sensing, nano- and biophotonics. 


\section{A Appendix}

The appendix to chapter 3 is structured in six sections. Section 3.A.1 contains a detailed technical description of the experimental setup and the optimization algorithm. In Sec. 3.A.2 we provide a detailed description of the experiment, followed by numerical simulations for the pulse duration of the optimized pulses. In Sec. 3.A.3 we analyze the influence of noise on the enhancement. In Sec. 3.A.4 we derive the correction factor for the non-uniform illumination of the spatial light modulator. Section 3.A.5 contains the sample characterization based on the time-resolved transmission measurement. In Sec. 3.A.6 we experimentally demonstrate that the resulting spatial focus from the wavefront shaping experiment is limited to the size of a speckle spot of the transmitted field.

\section{A.1 Experiment}

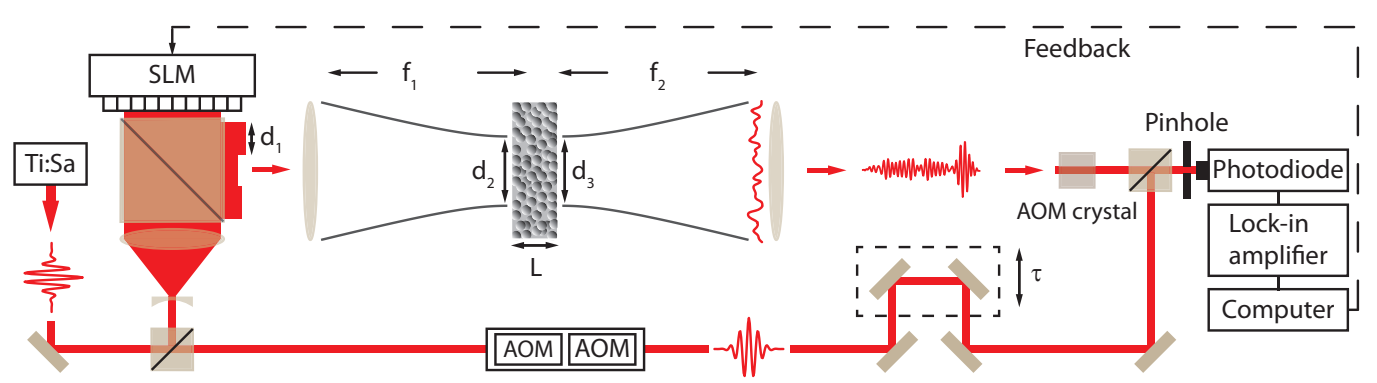

Figure 3.6: Experimental setup (see text).

\section{Wave-front shaping interferometer}

The polarized ultra-short pulses are provided by a Ti:Sapphire laser (Tsunami, Spectra Physics) at a central wavelength of $795 \mathrm{~nm}$ with a pulse duration of $64 \mathrm{fs}$ at a repetition rate of $80 \mathrm{MHz}$.

In the signal arm, the beam is first expanded to illuminate the pixel array (16 by $12 \mathrm{~mm}$ ) of the phase-only spatial light modulator (SLM)(X10468, Hamamatsu). The SLM pixels are grouped into $\mathrm{N}$ independent segments each of which induces a controllable phase shift $\Delta \Phi_{i}$, which is within $2 \%$ constant over the bandwidth of the laser. The reflected modulated wavefront is separated from the incoming beam by a beam splitter and subsequently focused onto the sample $\left(f_{1}=100 \mathrm{~mm}\right)$. The scattering medium is a $L=13.5 \mu \mathrm{m}$ thick layer of titania pigment (DuPont R902) spray-coated on a BK7 substrate with a transport mean free paths of $l_{t}=1 \mu \mathrm{m}$ for light of the laser wavelength. For the segmentation of the SLM into 300 square segments, light from a single segment $\left(d_{1}=0.8 \mathrm{~mm}\right)$ is focused into a spot of $d_{2} \approx$ $100 \mu \mathrm{m}$ on the sample surface, which is also the upper limit of the spot size for the complete incoming wave-front, independent of the applied spatial phases. The size of the diffuse spot on the backside of the sample is given by $d_{2} \approx d_{3}$, the influence of the diffusion on $d_{3}$ is negligible since $d_{2} \gg L$. The transmitted light appears as spatiotemporal speckle pattern. At the position of the collimating lens $\left(f_{2}=\right.$ $200 \mathrm{~mm}$ ), the speckle size is $d_{s} \approx 3 \mathrm{~mm}$. 
In the reference arm, two acousto-optical modulators (AOM) shift the light frequency by $40 \mathrm{kHz}$, which enables heterodyne detection of the transmitted pulses [124].

The interference between the light of both arms is measured with a photodetector. In front of the detector a pinhole (diameter $500 \mu \mathrm{m}$ ) selects light of a single speckle spot, corresponding to the forward scattered light. In Fig. 3.1 of the manuscript the pinhole is shown directly after the lens for a clearer illustration; the actual distance of the pinhole to the lens does not affect the selection of the speckle; the position in front of the detector in more practical - only the light from the reference arm arrives at the detector, which also contributes to the interference. A dual-output lock-in amplifier demodulates the signal from the photodetector giving out both the amplitude and the relative phase of the cross-correlation. In order to only measure the relative phase changes caused by the sample and the SLM, the material dispersion in the AOMs is compensated by a crystal of the same material (AOM crystal) in the signal arm before the combining beam splitter. The limited bandwidth of the AOM assembly narrows the spectrum of the reference pulses by $13 \%$. The time-resolution of the setup, given by the duration of the input pulses, therefore increases slightly by the same percentage. Amplitude and phase of the speckle pulses will not change significantly within this duration. The heterodyne signal, the amplitude of the fieldfield cross-correlation, is therefore effectively an instantaneous measurement of the transmitted field amplitude at the delay time $\tau$.

In the following section we describe the optimization algorithm which addresses the SLM during the wavefront shaping experiment.

\section{Optimization algorithm}

The optimization algorithm sequentially shifts the phase value of each segment $\Delta \Phi_{i}$ from $+0 \pi$ to $+2 \pi$ in eight steps and sets the phase value back to its start value before addressing the next segment. For each step and each segment the amplitude of the heterodyne signal is recorded from the lock-in amplifier. After that, for each pixel the amplitude versus phase shift is fitted with a cosine function to determine the phase value $\Delta \Phi_{i, \text { opt }}$ with the maximum feedback signal. Finally, the set of optimal phase values $\Delta \Phi_{i, \text { opt }}$ is written to the SLM to form the optimized pulse.

\section{A.2 Duration of the optimized pulses}

In the experiments we have observed that the optimized pulses are lengthened in comparison with the input pulses. This increase in pulse duration can be caused either by spectral narrowing or by spectral dispersion or, in the general case, by a combination of both effects. In this section we will show that both effects play a role in our experiment, and that their relative contribution depends on the number of segments used in the optimization. In the following we first give a rigorous analytical description of the experiment. Then we present numerical simulations, which describe how the spectrum and the spectral phase of the optimized pulses depend on the segmentation of the spatial light modulator. 


\section{Heterodyne feedback signal - analytical description}

The heterodyne signal $R(\tau)$ serves as the feedback signal for the optimization algorithm. It is proportional to the modulus of the field-field cross-correlation $C(\tau)$ of the pulse transmitted through the signal arm of the Mach-Zehnder interferometer, $E_{\text {sig }}$, and the reference pulse $E_{\text {ref }}$, where $\tau$ is the time delay of the reference pulse. It is given by

$$
R(\tau)=a|C(\tau)|=\left|F^{-1}\left\{E_{\mathrm{sig}}(\omega) E_{\mathrm{ref}}^{*}(\omega)\right\}\right|
$$

where $a$ is an experimental proportionality factor, $F^{-1}$ denotes the inverse Fourier transform. We can separate each contribution into the ingoing light from the source and a transfer function $H(\omega)$, which describes the phase and amplitude changes induced in the respective interferometer arm,

$$
C(\tau)=F^{-1}\left\{\left|E_{\text {source }}(\omega)\right|^{2} H_{\text {ref }}^{*}(\omega) H_{\text {sig }}(\omega)\right\} .
$$

Characteristic for linear interferometry, $C(\tau)$ is only dependent on the intensity spectrum of $E_{\text {source }}$, but independent of its phase. In our experimental realization, $H_{\text {ref }}(\omega)$ is dominated by the acousto-optical modulators (AOMs), which introduce dispersion and slightly narrow the spectrum. $H_{\text {sig }}(\omega)$ contains the contribution of the spatial light modulator (SLM), the opaque scattering medium and the lead molybdate crystal. The latter precisely compensates the dispersion of the AOMs, such that we can neglect the contribution of the crystal in the signal arm and assume $H_{\text {ref }}(\omega)$ to be real. The light transmission from the $\mathrm{N}$ segments of the SLM through the sample is described by the sum over $\mathrm{N}$ independent, complex transmission matrix elements $t_{i}(\omega)$, which can be altered by an individual phase shift $\Delta \Phi_{i}$. In fact, the phase shift is approximately linearly dependent on frequency, but can be considered constant over the bandwidth of the laser in our experiment,

$$
C(\tau)=F^{-1}\left\{\left|E_{\text {source }}(\omega)\right|^{2}\left|H_{\text {ref }}(\omega)\right| \sum_{i=1}^{N} t_{i}(\omega) e^{i \Delta \Phi_{i}}\right\} .
$$

Writing out the Fourier transformation and splitting up $t_{i}(\omega)$ in amplitude and phase,

$$
C(\tau)=\int_{-\infty}^{+\infty} d \omega\left|E_{\text {source }}(\omega)\right|^{2}\left|H_{\text {ref }}(\omega)\right| \sum_{i=1}^{N}\left|t_{i}(\omega)\right| e^{i\left[\Phi_{i}(t)+\Delta \Phi_{i}\right]} e^{i \omega \tau}
$$

we can see that the optimization at a selected delay time $\tau=\tau_{\text {opt }}$ maximizes the integral over the linear combination of the phase terms.

\section{Spectral narrowing}

From Eq. 3.6 we can deduce that the optimization will intrinsically lead to spectral narrowing of the focused pulses. The optimal set of phases $\Delta \Phi_{i}$ maximizes the integral over the linear combination of the phase terms. This integral is weighted by the spectral amplitude of the reference pulse and of the randomly transmitted pulses. Because the central part of the input spectrum, where this weight is highest, 
contributes more to the feedback signal than the wings of the spectrum to a smaller extent, we can expect that the optimization will lead to spectral narrowing.

We calculated the weighting factor $\left|E_{\text {source }}(\omega)\right|^{2}\left|H_{\text {ref }}(\omega)\right|$ for our experimental parameters. Assuming that the spectral amplitude is only filtered by this weighting factor, without any further spectral distortion, we calculate that the final pulse has a Fourier-limited pulse duration $\Delta t=115$ fs. This calculated results perfectly matches the observed pulse duration in the experiment.

This simplistic calculation assumes that the WFS optimization has fully compensated for fluctuations of the spectral phase. From our experimental data alone we are not able to distinguish this ideal situation from the case that the same pulse duration results from a slightly broader spectrum, but with remaining phase distortions. In the following we will present numerical simulations which show how the optimization converges to a Fourier-limited focus as a function of the number controlled SLM segments.

\section{Spectral narrowing as a function of $N$ - simulations}

General procedure. $\mathrm{N}$ temporal speckle pulses are generated, each representing the contributed field $E_{i}(t)$ at the detector, originating from one of the $\mathrm{N}$ segments on the SLM. For each randomly generated temporal speckle pulse the phase shift is determined which optimizes the heterodyne signal for a reference pulse delayed to the time $\tau_{\text {opt }}$. Afterwards, the sum of all contributions with the respective optimal phase shift is calculated. Finally the width of the peak in the heterodyne signal $\Delta \tau_{\text {opt }}$ and the pulse duration $\Delta t_{\mathrm{opt}}$ are determined.

Description step by step. In the following the simulation is described in detail. We use the measured spectra of the reference and the signal pulse at the detector to simulate the pulses:

1. Generate a Gaussian shaped input pulse $E_{\text {in }}(\omega)$ and a Gaussian shaped reference pulse $E_{\text {ref }}(\omega)$ with flat spectral phase using the values of the experimentally measured bandwidth.

2. Generate a frequency-dependent transmission coefficient $t_{i}(\omega)$, which connects the field on the SLM segment $i$ and the detector. For this, the algorithm described in chapter 4.1 is applied, using the experimental parameters obtained for the sample characterization described in appendix 3.A.5.

3. The transmitted field originating from segment $i$ is $E_{i}(\omega)=E_{\text {in }}(\omega) t_{i}(\omega)$.

4. Calculate the cross-correlation for the contribution $C_{i}(\tau)=F^{-1}\left\{E_{\mathrm{ref}}(\omega) E_{i}^{*}(\omega)\right\}$ by fast Fourier transform.

5. The optimized contribution is calculated $E_{i, \mathrm{opt}}(t)=E_{i}(t) e^{-i \Delta \Phi_{i}}$, where $\Delta \Phi_{i}$ is the phase of $C_{i}\left(\tau_{\mathrm{opt}}\right)$.

6. Repeat Steps 1-6 for $\mathrm{N}$ times, virtually creating $\mathrm{N}$ pulses originating from $\mathrm{N}$ segments on the SLM. 
7. Create the optimized output pulse $E_{\text {out }, \text { opt }}(t)=\sum_{i} E_{i, \text { opt }}(t)$.

8. Create the Fourier-limited pulse from the optimized output pulse, $E_{\text {out,opt,Fl }}$.

9. Calculate the cross-correlation $C_{\text {out,opt }}$.

10. Determine the intensity pulse duration $\Delta t_{\text {opt }}$ (FWHM) from $\left|E_{\text {out,opt }}(t)\right|^{2}$, the pulse duration of same pulse compressed to the Fourier-limit $\Delta t_{\text {opt,Fl }}$ from $\left|E_{\text {out,opt,Fl }}(t)\right|^{2}$, and the width of the peak of the heterodyne signal $\Delta \tau_{\text {opt }}$ from $\left|C_{\text {out }, \text { opt }}(\tau)\right|$.

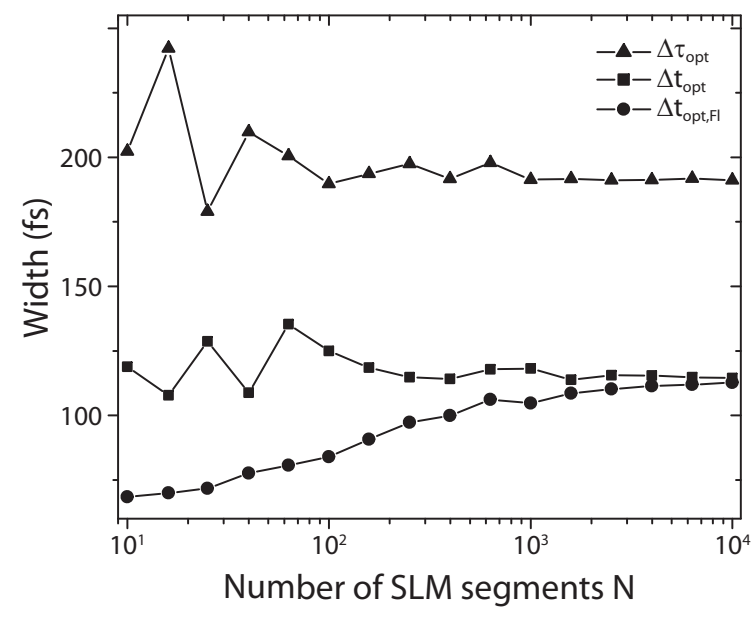

Figure 3.7: Results from numerical simulations. Width (FWHM) of heterodyne signal $\Delta \tau_{\text {opt }}$ (triangles) and pulse duration $\Delta t_{\text {opt }}$ (squares) of the optimized output pulse, and the Fourier-limit $\Delta t_{\mathrm{opt}, \mathrm{Fl}}$ of the optimized pulses (circles).

Results. We performed a series of the simulation for increasing number of segments $N$. The pulse durations obtained from simulations are shown in Fig. 3.7. The width of the peak of the heterodyne signal (FWHM) converges to a fixed value of $\Delta \tau_{\text {opt }}=$ $(192 \pm 1) f s$. The duration of the optimized pulses is merely constant, fluctuating around a mean value of $\Delta t_{\mathrm{opt}}=(115 \pm 1) \mathrm{fs}$, with fewer fluctuations for larger $N$. The results for $\Delta \tau_{\mathrm{opt}}$ and $\Delta t_{\mathrm{opt}}$ perfectly match the result from the experiment.

The results show that the Fourier-limited duration of the optimized pulse increases with the number of pixels and converges to the value of $\Delta t_{\mathrm{opt}}$. The gap between the duration of the optimized pulses and their Fourier-limited duration indicates that the optimized pulses are lengthened by a non-flat phase for a low number of segments. For an increasing number of segments $\Delta t_{\text {opt }}$ and $\Delta t_{\mathrm{BW}}$ converge, which means that the optimized pulses are Fourier-limited, with a somewhat narrower spectrum than the input pulses. We conclude that in the case of our experiment with $N=300$ segments, the optimized pulses are close to the Fourier-limit. 


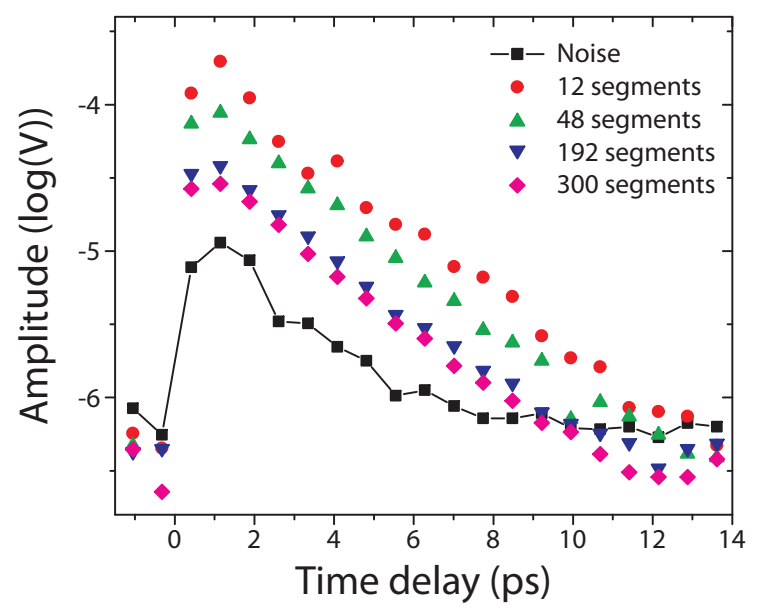

Figure 3.8: Average contribution of a single SLM segment to the total heterodyne signal for a different number of segments (coloured symbols). For long delay times the contribution drops below the noise level (black line), which reduces the effectiveness of the optimization.

\section{A.3 Influence of noise}

In the experiment we have observed that the enhancement $\alpha$ decreases at long delay times, which we attribute to noise. In this section, we quantitatively analyze this effect.

The influence of the noise level on the enhancement $\alpha$ is illustrated in Fig. 3.8, comparing the noise on the heterodyne signal (line with squares) with the averaged contribution from a single SLM segment to the total detected signal (symbols). The noise level (black squares) was determined from the standard deviation of a large number of measurements with the same random phase pattern, where the heterodyne signal is measured 300 times at each time delay of the reference pulse. The average contribution from a single SLM segment (coloured symbols) was determined for different number of segments $\mathrm{N}$ from the measured average oscillation of the recorded heterodyne signal when the phase shift $\Delta \Phi_{i}$ of the segments was cycled during the optimization procedure.

For time delays above about $9 \mathrm{ps}$, the average contributions from the optimization with 300 segments drops below the noise level, the measurements with lower $\mathrm{N}$ following at longer time delays. In this regime the optimal phase shift is not found any more, which explains well the drop of enhancement $\eta$ observed in the experiment.

\section{A.4 Enhancement factor and correction for non-uniform illumination of the spatial light modulator}

In this section we provide an analytical description of the enhancement factor $\alpha$, including the correction factor $\sigma$, which accounts for non-uniform illumination of the spatial light modulator (SLM).

At a given time t, we assume a circular complex Gaussian distribution for the 
electric field contribution by segment $i$,

$$
p\left(E_{i}\right)=\frac{1}{2 \pi A_{i}^{2}} e^{-\frac{\left|E_{i}\right|^{2}}{2 A_{i}^{2}}}
$$

with an average (root mean square) field amplitude $\left\langle\left|E_{i}\right|\right\rangle_{\mathrm{rms}}=\sqrt{2} A_{i}, A_{i}$ being proportional to the input field amplitude. The overall time dependence of $A_{i}$, which we neglected here, is identical to the behaviour of the amplitude shown in Fig. 3.9. For a uniform illumination of the SLM all $A_{i}$ are identical, in our case we have to take the Gaussian profile of the beam into account.

For $\mathrm{N}$ segments, the probability distribution of the non-optimized combined field is given by [131]

$$
\begin{aligned}
p\left(E_{\mathrm{rnd}}\right) & =\frac{1}{2 \pi N\left(\sum A_{i}^{2}\right)} e^{-\frac{\left|E_{\mathrm{rnd}}\right|^{2}}{2\left(\sum A_{i}^{2}\right)}} \\
& =\frac{1}{2 \pi N\left\langle A^{2}\right\rangle} e^{-\frac{\left|E_{\mathrm{rnd}}\right|^{2}}{N\left\langle A^{2}\right\rangle}}
\end{aligned}
$$

with an average non-optimized amplitude $\left\langle\left|E_{\text {rnd }}\right|\right\rangle_{\mathrm{rms}}=\left(2 N\left\langle A^{2}\right\rangle\right)^{1 / 2}$, with $\left\langle A^{2}\right\rangle$ being averaged over all the amplitudes $A_{i}$ on the SLM surface.

After an optimization with $\mathrm{N}$ segments, the average outgoing field is

$$
\begin{aligned}
\left\langle\left|E_{\mathrm{opt}}\right|\right\rangle_{\mathrm{rms}} & =\left(\int_{-\infty}^{+\infty} \prod_{i=1}^{N} \frac{1}{2 \pi A_{i}^{2}} e^{-\frac{\left|E_{i}\right|^{2}}{2 A_{i}^{2}}}\left(\sum_{i=1}^{N}\left|E_{i}\right|\right)^{2} d E_{1} \ldots d E_{N}\right)^{\frac{1}{2}} \\
& =\left(2 N\left\langle A^{2}\right\rangle+\frac{N(N-1)}{2} \pi\langle A\rangle^{2}\right)^{\frac{1}{2}}
\end{aligned}
$$

The average enhancement is consequently defined by

$$
\langle\alpha\rangle=\frac{\left\langle\left|E_{\mathrm{opt}}\right|\right\rangle_{\mathrm{rms}}}{\left\langle\left|E_{\mathrm{rnd}}\right|\right\rangle_{\mathrm{rms}}}=\left(1+\frac{\langle A\rangle^{2}}{\left\langle A^{2}\right\rangle} \frac{\pi}{4}(N-1)\right)^{\frac{1}{2}} \simeq\left(\frac{\langle A\rangle^{2}}{\left\langle A^{2}\right\rangle} \frac{\pi}{4} N\right)^{\frac{1}{2}}
$$

which reduces to $\left(\frac{\pi}{4} N\right)^{1 / 2}$ if we assume a uniform illumination of the SLM surface such that $A_{i}$ are equal. To determine $\sigma=\left(\langle A\rangle^{2} /\left\langle A^{2}\right\rangle\right)^{1 / 2}$ for our experiment, we determined the field distribution on the SLM surface by measuring the beam dimensions in front of the device, resulting in $\sigma=0.90$. The average intensity enhancement $\eta[13]$ is given by $\eta=\alpha^{2}$.

\section{A.5 Time-resolved transmission measurements}

In this section we analyze the average time-resolved transmission through the scattering sample. From the measurements we calculate the diffusion constant and the $C_{1}$ frequency correlation, which is used in the simulations in appendix 3.A.2.

The scattering medium consist of a layer of titania pigment (DuPont R902, thickness $L=13.5 \mu \mathrm{m})$ spray-coated on a BK7 substrate. The transport mean free path 


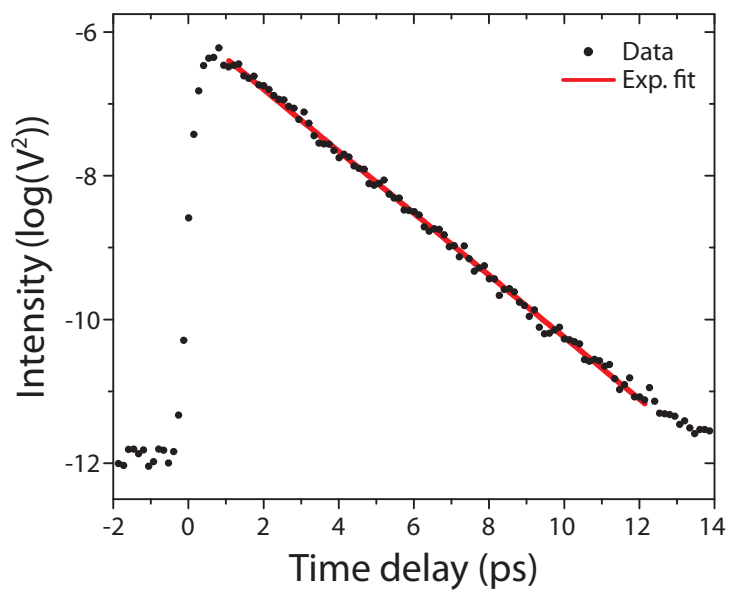

Figure 3.9: Average amplitude of field cross-correlation of a non-optimized pulse as a function of the time delay of the reference pulse, averaged over 50 random phase patterns (black dots). The data has been fitted with a single exponential decay (red line). The origin was defined by the maximum of a reference measurement without a scattering sample in the optical path.

in this material is $l_{t}=1 \mu \mathrm{m}$ measured at $795 \mathrm{~nm}$ [123].

Figure 3.9 (black dots) shows the average intensity, calculated by the square of the average non-optimized heterodyne signal, as a function of the time delay. The averaging has been performed over 50 random phase patterns. In the regime of diffusive light transport, the long time behaviour of the average amplitude can be described by a single exponential decay: $I(t)=I_{0} e^{-t / \tau_{d}}[117]$. The time constant $\tau_{d}$ is the decay time as defined by Eq. 2.22. Figure 3.9 (red line) shows the fit of the data by the model on the $\log$ arithmic scale $\log I(t)=\log I_{0}-t / \tau_{d}$, giving $\tau_{d}=1,00 \pm 0,01 \mathrm{ps}$. The data is described well by a single exponential decay, indicating a diffusive light transport through our sample. We calculated the diffusion constant $D \approx L^{2} /\left(\tau_{d} \pi^{2}\right)=18,5 \mathrm{~m}^{2} / \mathrm{s}$ and a $C_{1}$ frequency correlation $\delta \omega_{\text {corr }} \approx 2.92 /\left(\pi \tau_{d}\right)=0.92 \cdot 10^{12} \mathrm{~s}^{-1}[117]$.

\section{A.6 Spatial extent of the focal spot}

In this section we show that the spatial extent of the spatiotemporal focus is limited to the typical size of a speckle spot of the transmitted speckle field.

For this measurement the optical setup as shown in Fig. 3.6 was slightly extended. We placed a flip mirror between the combining beam splitter and the pinhole which directs the transmitted light to a CCD camera (AVT Dolphin F145B). The camera was positioned such that the CCD chip had the same distance to the back interface of the scattering medium as the pinhole. Without the flip mirror in the optical path the field in an area of a single speckle spot is detected with time-resolution. With the flip mirror several speckle spots can be spatially resolved by the camera without time-resolution.

We performed the following experiment. First, we recorded the speckle pattern on the camera with a random phase distribution on the SLM segments. Afterwards we directed the light to the pinhole and optimized the amplitude at the delay time 

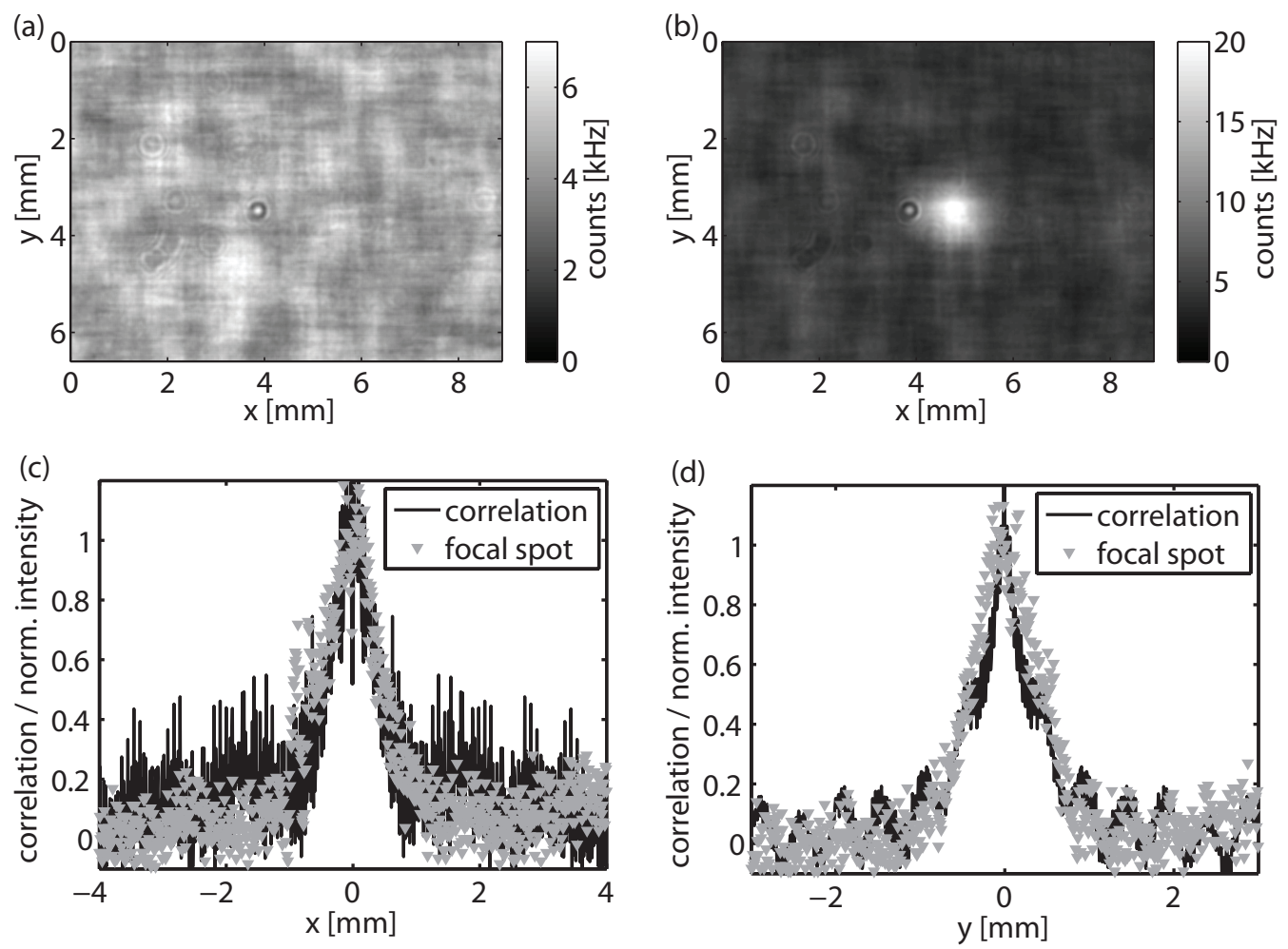

Figure 3.10: (a) Camera image of the transmitted speckle field with a random wavefront incident on the medium. (b) Camera image of the transmitted light after a wavefront shaping experiment with a temporal focus comparable to Fig. 3.2c. The light is spatially focused. $(\mathrm{c}+\mathrm{d})$ Plot of the autocorrelation of the speckle pattern before wavefront shaping (black line) with a cross-cut through the maximum of the focal spot after wavefront shaping (grey triangles). 
1 ps with a wavefront segmented into 192 segments. The peak amplitude at the focal time is enhanced by a factor of $\alpha=12$, comparable to the result summarized in Fig. 3.3. After the WFS experiment, we directed the light to the camera again and recorded the speckle field.

The results are illustrated in Fig. 3.10. Before the WFS experiment the camera image shows a random speckle pattern (Fig. 3.10a). After the wavefront shaping experiment the light is spatially focused (Fig. 3.10b). We calculated the autocorrelation function of the random speckle pattern and compared it to the size of the focal spot after WFS. Both in the $\mathrm{x}$ - and the $\mathrm{y}$-direction of the images, the speckle correlation and the focal spot are of the same width with a full width at half maximum of FWHM $=1 \mathrm{~mm}$ at the camera position (Fig. $3.10 \mathrm{c}+\mathrm{d}$ ).

In conclusion, the spatial extent of the spatiotemporal focus is limited to the size of a speckle spot. 



\section{Analysis of linear and nonlinear feedback methods based on numerical simulations}

In the first part of this chapter, we describe a general way to simulate the speckle wave field scattered from a random scattering medium. In the second part, we use the approach to generate random speckle pulses for simulations of short-pulse wavefront shaping experiments. We introduce the concept of short-pulse wavefront shaping with a slow nonlinear detector. We show based on simulations that temporal focusing is achieved even when the nonlinear detector response is slower than the pulse duration. The experimental realization of this concept will be presented in chapters 5 and 6 . The approach is investigated in terms of achieved pulse duration and the convergence behaviour of the wavefront shaping optimization. The particular case of the investigated optimization of a nonlinear feedback signal illustrates a benefit of the simulations: We find that the result of the optimization depends on the starting conditions. Since we can readily simulate a large number of wavefront shaping optimizations, we can gain valuable information on the statistics of the optimization results which are otherwise hard to obtain from an experiment.

\subsection{Generation of speckle pulses}

\subsubsection{Algorithm}

In this section we describe the generation of the random speckle pulses, which are the basis for the simulations in the following sections. The algorithm generates a discrete time signal $E_{i}\left[t_{i}\right]$ which represents the speckle pulse amplitude from transmission through a random medium over a uniformly spaced times $t_{i}=i \Delta t, i=1,2, \ldots, N$. The sampling rate $\Delta t^{-1}$ is thereby set such that an optical cycle in the relevant frequency range is sufficiently sampled and the sampling interval $t_{\max }=N \Delta t$ is 

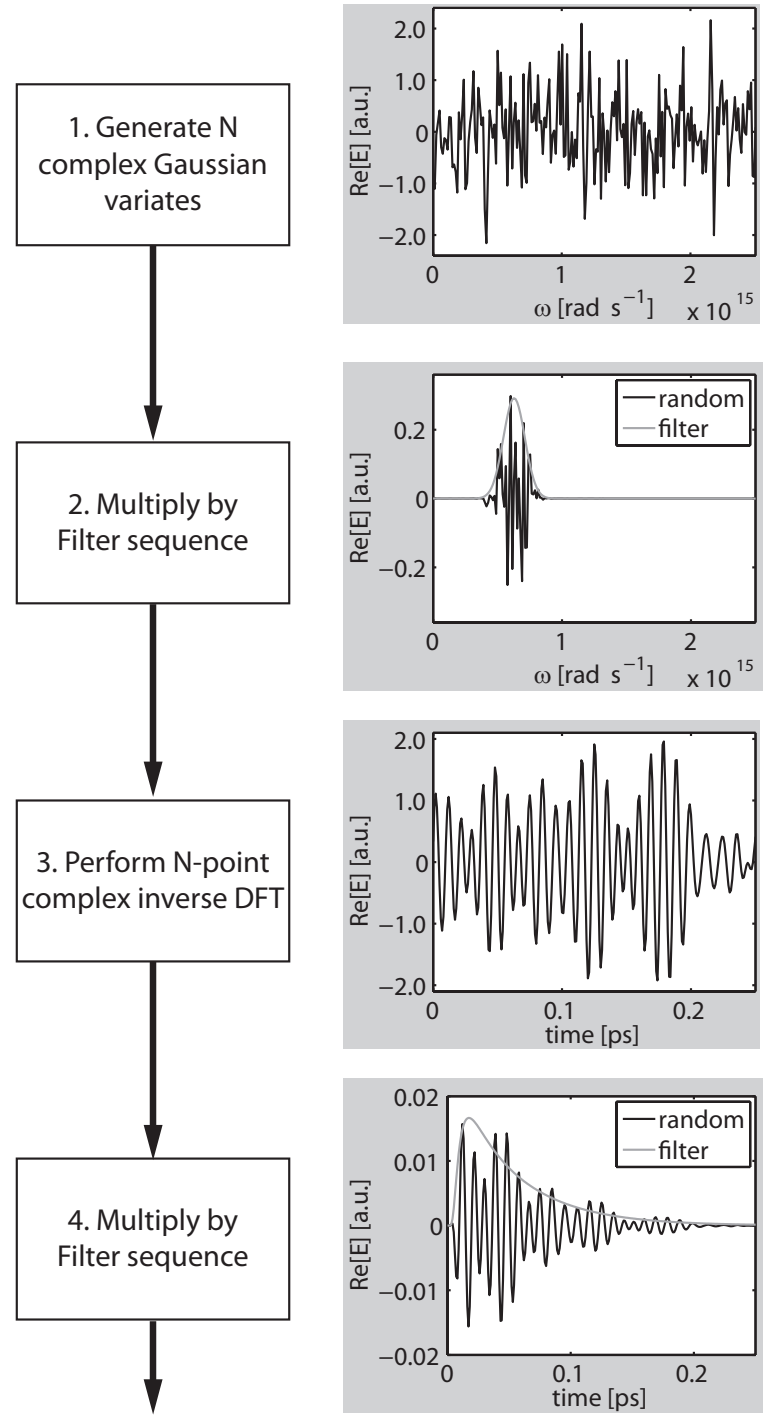

Figure 4.1: Main steps of the speckle pulse algorithm (right), together with an example data set which is illustrated after each step (left). 1. The real part of generated set $E_{i}$ is plotted over the frequency $w_{i}$. The values of $E_{i}$ are uncorrelated and extend over the full spectrum. 2. The real part of the filtered set $E_{i}$ is plotted at each frequency $w_{i}$, together with the used filter $S_{i}$, which represents the Gaussian shaped laser spectrum. 3. The real part of the time series after Fourier transform. A random pulse train is visible, which extends over the full time range of the calculation. 4. The real part of the of the time series after filtering is plotted at each time $t_{i}$, together with the filter $I_{i}$. Now the field represents a random speckle pulse, which shows the typical exponential decay. Note that the frequency and bandwidth of the filter $S_{i}$ has been chosen in the terahertz regime with a large bandwidth for and illustrative purpose. For the same reason, the plots on the right-hand side do not show the full sequences, but rather are cut to highlight respectively the range of lower frequency or earlier times. 
chosen such that the dynamics of the modeled system are fully described. The main steps of the algorithm are:

1. Generation of 'white noise' in the frequency domain. Generate a set of N uncorrelated random numbers $E_{i}, i=1,2, \ldots, N$, where each element represents the complex electric field at the respective frequency $\omega_{i}=i 2 \pi / t_{\max }, i=1,2, \ldots, N$. The values of $E_{i}$ are drawn from a circular complex gaussian distribution [98], of which the mean value is zero and the variance is arbitrarily chosen to 1 .

2. The set $E_{i}\left[\omega_{i}\right]$ is multiplied element-wise by the filter $S_{i}\left[\omega_{i}\right]$ which represents the spectral amplitude function of the laser pulse. In the illustration in Fig. 4.1, a Gaussian shaped spectral function is used.

3. A N-point complex discrete Fourier transform transforms the signal into the time domain.

4. In the time domain, the sequence is again multiplied element-wise by a second filter $A_{i}\left[t_{i}\right]$, where the elements of $A$ represent the square root of the ensembleaveraged time-dependent (intensity) transmission of the system of interest. For a random slab $A$ is calculated by Eq. 2.18.

The performed algorithm is related to algorithms applied in telecommunications research [132]. In that research field, the random modulation of the magnitude of a signal is termed 'Rayleigh fading', referring to the underlying probability distribution for the process.

Before using the generated speckle pulses for simulations of wavefront shaping experiments, we verified that their speckle statistics agree with the predictions from theory.

\subsubsection{Statistical properties}

Here we show that the statistics of the simulated speckle pulses agrees with theory. We have illustrated the comparison of simulations and theory in Fig. 4.2. Fig. 4.2a+b show a simulated speckle pulse in the time domain and the frequency domain generated for parameters typical to our experiments. We generated $N=1000 \mathrm{such}$ random speckle pulses and numerically evaluated the average statistical properties of the transmission coefficients $t_{a b}(\omega)$ within the FWHM bandwidth of the Gaussian spectral amplitude (Fig. 4.2c-e). We determine the frequency-frequency correlation $C^{(1)}(\Delta \omega)$, the distribution of the group delay time $P\left(\widetilde{\phi}^{\prime}\right)$ and the distribution of the weighted delay time $P(\widetilde{W})$. Each quantity is plotted together with the theoretically values calculated by the respective Eqs. 2.34, 2.36 and 2.38. The simulations are in excellent agreement with theory.

\subsubsection{Summary and outlook}

In this first section we presented a generic method to simulate a random realization of the wave field scattered from a medium. The approach to generate speckle pulses is easy to implement and can be applied to a wide range of simulations in random 
systems. In this chapter we restrict ourselves to simulations of the transmission from independent incident to independent outgoing scattering channels. The approach can be extended to the multiple channels by incorporating the corresponding spatial correlation functions (see section 2.4.2).

In the following sections we use the presented algorithm to numerically investigate different WFS scenarios and make predictions relevant for the experiments which will follow in the chapters 5 and 6 . In a similar fashion the algorithm was already used
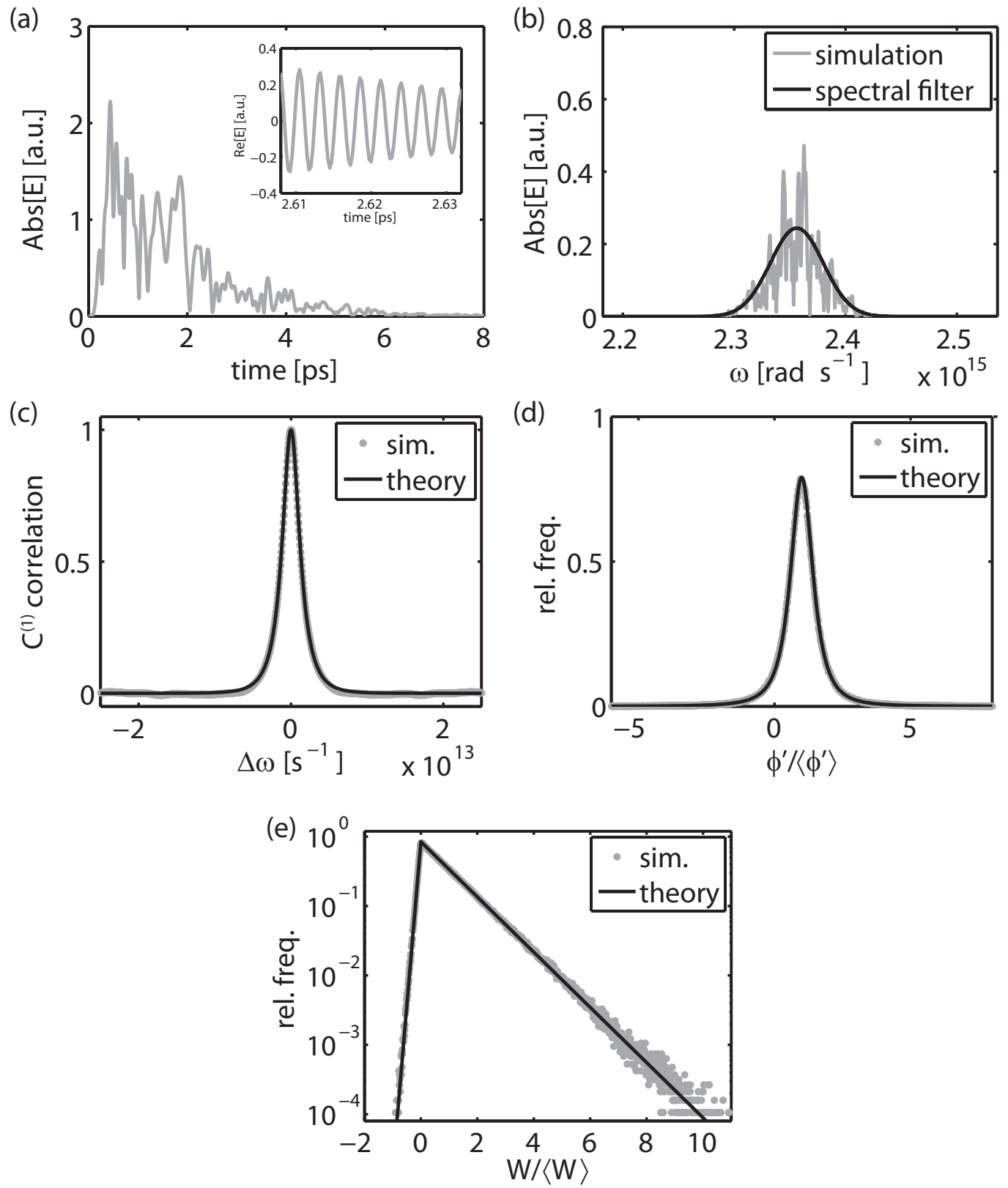

Figure 4.2: Simulated speckle pulses and statistical analysis. (a+b) Transmitted speckle field for a bandwidth limited pulse (duration $\Delta t=70 \mathrm{fs}$, center wavelength $\lambda=800 \mathrm{~nm}$ ) through a semi-infinite slab (thickness $L=20 \mu \mathrm{m}$, diffusion constant $D=58 \mathrm{~m}^{2} / \mathrm{s}$ ). (c-e) Average statistics of $N=1000$ speckle pulses: the $C_{1}$-correlation (c), the distribution of the group delay time $P\left(\widetilde{\phi^{\prime}}\right)$ (d) and the distribution of the weighted delay time $P(\widetilde{W})$ (e). 
for simulations in the preceding chapter (see appendix 3.A.2).

\subsection{Short-pulse WFS with a slow nonlinear detec- tor}

In this section we demonstrate by simulations that short-pulse wavefront shaping can achieve temporal focusing with slow detectors, if the detector has a response with a nonlinear dependency on the incident intensity.

In chapter 3 we demonstrated short-pulse wavefront shaping, where the timeresolved feedback signal was provided by a gating pulse. This approach is powerful, as it enables in principle a full characterization of the transmitted wave field and full control over the spatiotemporal focus. However, a time-resolved detection scheme in optics requires a relatively complex experimental apparatus, which is not always practical. Here, we pursue and alternative approach: We demonstrate by simulations that short-pulse wavefront shaping can achieve temporal focusing with slow detectors, if the detector has a response with a nonlinear dependency on the incident intensity. By 'slow' we here mean that the detector response time is slower than both the initial pulse duration of the laser source and the typical traversal time for the waves through the medium to the detector. The idea is related to frequency-domain pulse shaping techniques, where the adaptive optimization of a nonlinear feedback signal is used for pulse compression [133].

\subsubsection{Comparison of linear and nonlinear detection}

In the following we show that a nonlinear response of the slow detector is required for temporal focusing. We simulated two wavefront shaping experiments. For the first one the feedback signal from the target spot was generated by a slow detector response which scales linearly with the intensity of the incident light. In the second simulation, the slow detector had a nonlinear response. We performed the simulation for a scalar electric field. $N=300$ independent SLM segments were used. The undistorted pulse had a Gaussian spectral function around the center wavelength $\lambda=800 \mathrm{~nm}$ and a Fourier-limited pulse duration of $\Delta t=100 \mathrm{fs}$. The transmitted speckle pulses were generated for a semi-infinite slab of thickness $L=20 \mu \mathrm{m}$ and a diffusion constant $D=18.5 \mathrm{~m}^{2} / \mathrm{s}$. At the detector, each of the $N$ segments contributes the speckle pulse $E_{i}(t)$, which we generated by the algorithm described above in section 4.1.1. The linear detector response is calculated by

$$
S_{1}=\alpha_{1} \int_{-\infty}^{\infty}|E(t)|^{2} d t
$$

and the nonlinear second-order response is calculated by

$$
S_{2}=\alpha_{2} \int_{-\infty}^{\infty}\left|E^{2}(t)\right|^{2} d t .
$$


(a)

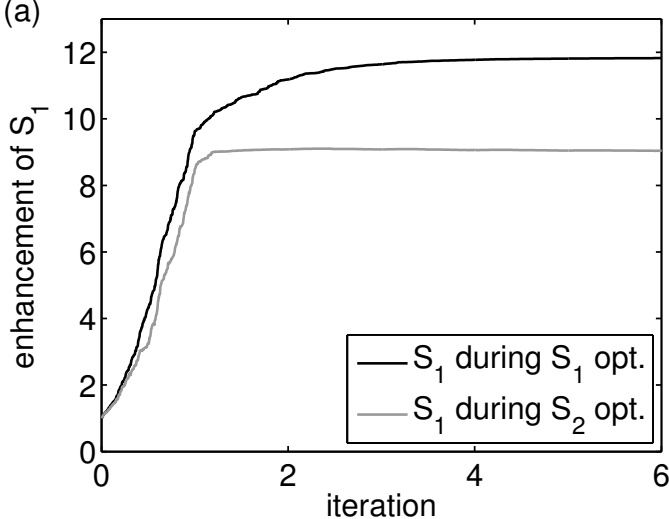

(c)
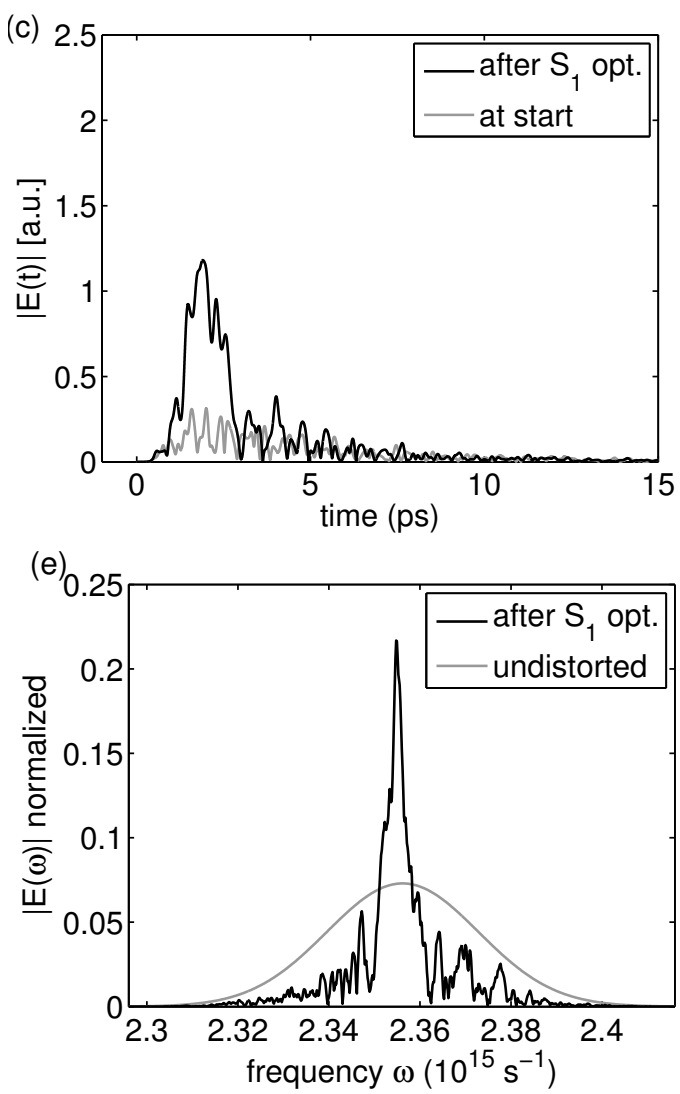

(b)

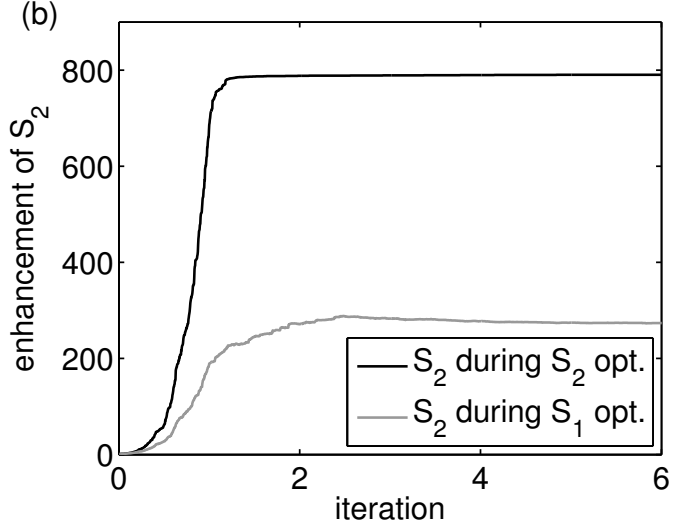

(d) 2.5

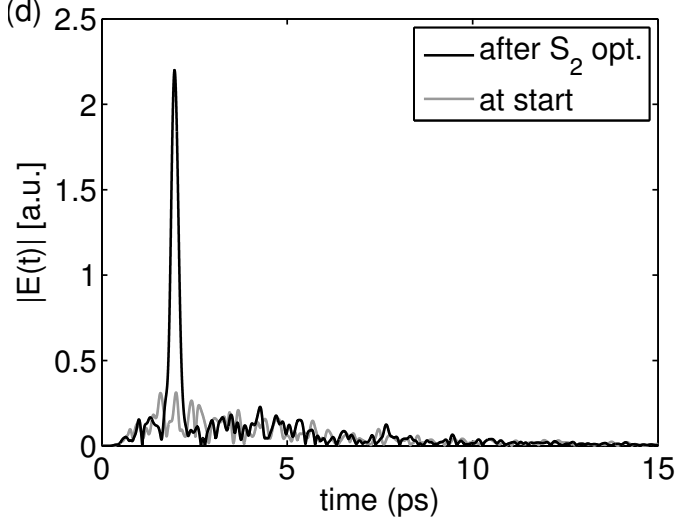

(f)

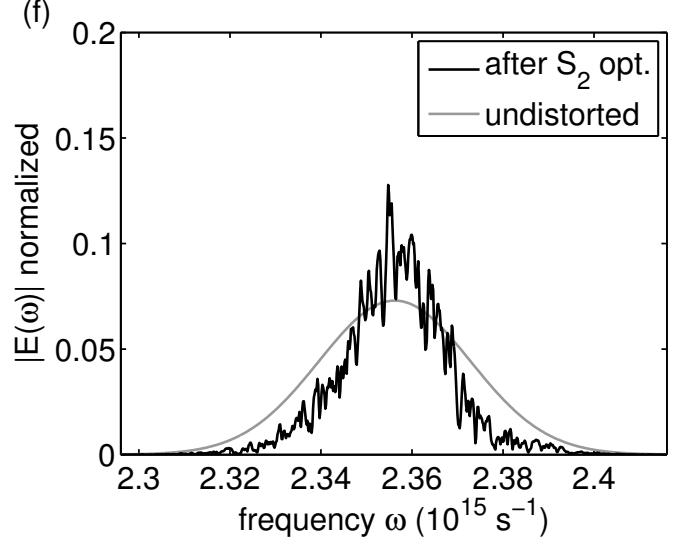

Figure 4.3: Results of two simulated wavefront shaping experiments in which either $S_{1}$ (Eq. 4.1) or $S_{2}$ (Eq. 4.2) are optimized. (a) Enhancement of $S_{1}$ when $S_{1}$ is feedback signal (black line) and when $S_{2}$ is feedback signal (grey line). (b) Corresponding plot for $S_{2}$. (c) Amplitude $|E(t)|$ at the detector at the start of the simulation (grey line) and after optimization of $S_{1}$ (black line). (d) Amplitude $|E(t)|$ at detector after optimization of $S_{2}$. (e) Spectral amplitude $|E(\omega)|$ of the unscattered pulse (grey line) and at the detector after optimization of $S_{1}$ (black line). Both spectra have been normalized to unity integrated intensity. (f) Spectral amplitude $|E(\omega)|$ after optimization of $S_{2}$. 
The proportionality constants $\alpha_{1}$ and $\alpha_{2}$ were chosen to be one in the simulations. For both wavefront shaping experiments we used the standard closed-loop sequential optimization algorithm performed to maximize $S_{1}$ and $S_{2}$ respectively. We performed multiple successive iterations of the algorithm for both cases.

The results of the simulations are illustrated in Fig. 4.3. In both simulations the feedback signals $S_{1}$ (see Fig. 4.3a) and $S_{2}$ (see Fig. 4.3b) increased monotonically and converged after a few repetitions of the algorithm. Here we define 'convergence reached' when the feedback signal during a full iteration of the algorithm increases by less than a fraction $1 / \mathrm{N}$. The optimization of $S_{2}$ converged within three iterations, whereas the optimization of the linear response $S_{1}$ converged only after the sixth iteration. For the optimization of the linear signal $S_{1}$, the non-optimized signal $S_{2}$ is also significantly increased (Fig. 4.3a). Vice versa, the optimization of $S_{2}$ is accompanied with an increase of the non-optimized signal $S_{1}$ (Fig. 4.3b). The focused fields differ significantly both in the time domain and by their spectral shape. After the optimization of $S_{1}$, the temporal amplitude is enhanced in a time interval of a few picoseconds around the maximum of the random transmission (Fig. 4.3c). A temporal focus on the time scale of the input pulses is not observed. The spectral amplitude changes randomly with frequency, with strongly enhanced components in center of the spectrum (Fig. 4.3e). After the optimization of $S_{2}$, the temporal amplitude is significantly enhanced only at a single point in time (Fig. 4.3d). The spectral shape is approximately Gaussian with far less random distortions than observed in the linear case (Fig. 4.3f). The width is narrower than the spectrum of the undistorted pulses, which we investigate in more detail in Sec. 4.2.3.

The results can qualitatively be explained in the following way. The optimization of the linear signal $S_{1}$ enhances the frequency components which contribute with the highest intensity at the detector. These are the components from the center part of the input spectrum with the highest transmission coefficients. Since highest transmission coefficient are most likely connected to a phase delay time around the average traversal time $\tau_{t}$ of the light through the medium (see Eq. 2.38 and Fig. 4.2d), the focused pulse is concentrated in a time window of duration on the order of $\tau_{t}$. However, the optimization of $S_{1}$ does not lead to a temporal focus on the order of the input pulse duration.

In contrast, the optimization of the nonlinear signal $S_{2}$ leads to a temporal focus. This makes sense intuitively as $S_{2}$ describes a two-photon process, which yields the highest outcome when all light arrives at the same time. In the following, we elaborate how this intuitive view applies to our experiment.

It is helpful to compare the short-pulse WFS approach to pulse shaping: In a pulse shaping process the total pulse energy is conserved and the intensity is only redistributed temporally. Earlier results proved that among the temporal distributions which can be synthesized by the pulse shaper, the shortest pulse yields the highest second-harmonic signal. In turn, an adaptive optimization of a second-order feedback signal starting from a random temporal pulse shape converges to a short pulse [133]. For the pulse shaping scenario the statement from above directly applies: The more the pulse is compressed the higher is the yield of the nonlinear process.

Our short-pulse wavefront shaping approach is substantially different from the pulse shaping scenario: Here the scattered light is redistributed spatially, and the 
(a)

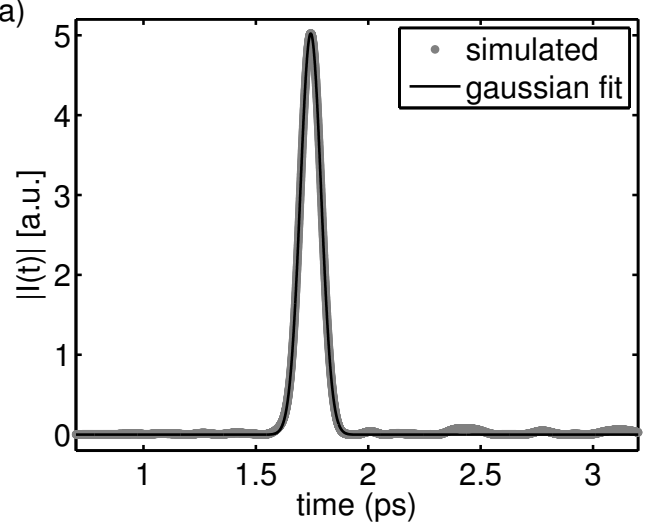

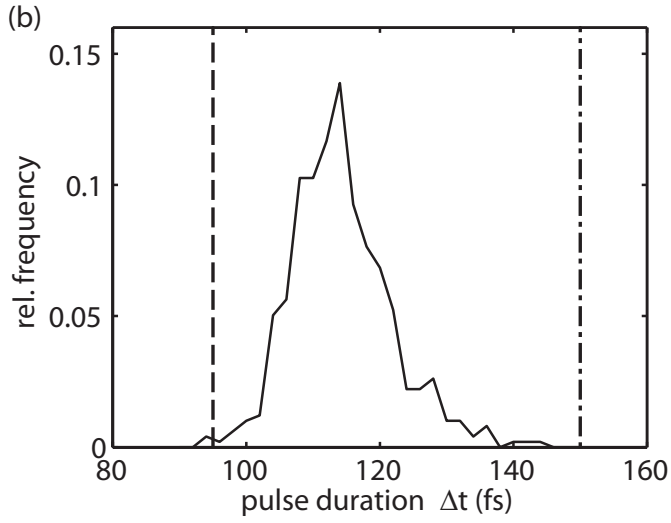

Figure 4.4: (a) Optimized pulse from simulation (grey dots) with Gaussian fit. (b) Distribution of focus pulse duration $\Delta t_{\text {focus }}$ from 500 optimizations, each with a different transmission matrix $T(\omega)$. (Parameters for the simulations: $N_{s}=300$ segments, $N_{i}=4$ iterations with sequential algorithm, input pulse duration $\Delta t=67 \mathrm{fs}$, center wavelength $\lambda=840 \mathrm{~nm}$, slab thickness $L=20 \mu \mathrm{m}$, diffusion constant $D=18.5 \mathrm{~m}^{\mathrm{s}} / \mathrm{s}$ )

temporal distribution of the intensity at one spatial coordinate changes only as a consequence of the spatial redistribution of the scattered light. For the purpose of spatial focusing, the speckle wave field is shaped to interfere constructively at one point in space. The result of the optimization of the time-integrated linear feedback $S_{1}$ shows that this spatial focusing is not necessarily linked to forming a short pulse. We can additionally focus the light temporally exploiting the limited coherence time of the speckle field as demonstrated in chapter 3: The focused light only interferes constructively at one point in space and within the coherence interval.

We compare two short-pulse WFS gedankenexperiments to show why the optimization of $S_{2}$ leads to temporal focusing: In the first one, all controlled channels of the wavefront are used to focus the pulse at one point in time and space. In the second gedankenexperiment, we focus at the same spatial coordinate, but dedicate respectively half of the segments to two separate points in time. Two separate subpulses are formed, with a peak intensity of about half the height compared to the peak in the first experiment. Applied to the nonlinear detector described in Eq. 4.2, the pulse from the first experiment will yield a signal about two times higher signal than the double-pulse from the second experiment. If the number of separate temporal foci would be increase from two, the difference would only be higher. From this gedankenexperiment we can conclude that the adaptive optimization of the nonlinear feedback will be biased to form a single temporal focus.

The resulting spectrum can be explained by two counter-balancing effects: Similar to the linear case, the spectral amplitude is narrowed compared to the input spectrum as the center part contributes more efficiently to the focus. The narrowing is limited by the effect that temporal concentration requires the maintenance of a broad spectral function. A quantitative analysis of the pulse duration and the arrival time of the temporal focus is given the following Sec. 4.2.3. 


\subsubsection{Pulse duration}

In this section we investigate the pulse duration which is achieved by short-pulse WFS with a slow nonlinear detector.

We simulated a series of optimizations with a nonlinear detector analog to the preceding section for many sample realizations. The simulation parameters are listed in the caption of Fig. 4.4. Graph (a) of the figure shows a typical pulse after the optimization (grey dots). The pulses are well described by a Gaussian function, and we applied a Gaussian fit (black line) to the data to determine the focus pulse duration $\Delta t_{\text {focus }}$. Figure $4.4 \mathrm{~b}$ illustrates that the distribution of the focus pulse duration $\Delta t_{\text {focus }}$ resulting from all simulations is spread over a finite interval.

In the following we derive an upper and a lower limit of the distribution of $\Delta t_{\text {focus }}$ and discuss the underlying mechanism of the spectral narrowing observed in Fig. 4.3. In chapter 3, we have already seen spectral narrowing occur in the short-pulse WFS experiment with optical gating. Because the optical gating signal is linear, the focus pulse duration deterministically converges to a sharp distribution around a finite value which can be exactly calculated (see appendix 3.A.2).

Here, the nonlinear feedback signal $S_{2}$ is optimized. With Eq. 2.26 the expression for $S_{2}$ (Eq. 4.2) can be rewritten as

$$
\begin{aligned}
S_{2} & =\alpha_{2} \int_{-\infty}^{\infty}\left|\left(\mathcal{F}^{-1}\left\{\sum_{a=1}^{N} E_{0}(\omega) t_{a b}(\omega) e^{i \phi_{a}}\right\}\right)^{2}\right|^{2} d t \\
& =\alpha_{2} \int_{-\infty}^{\infty}\left|\left(\sum_{a=1}^{N} E_{0}(t) \otimes t_{a b}(t) e^{i \phi_{a}}\right)^{2}\right|^{2} d t
\end{aligned}
$$

The algorithm optimizes $S_{2}$ by means of the set of phases $\phi_{a}$. Since the $C^{(1)}$ frequencycorrelation of the transmission coefficients $t_{a b}(\omega)$ is smaller than the bandwidth of the input spectrum $E_{0}(\omega)$, not all frequency components can be optimally transmitted simultaneously. The average probability of a narrow frequency-band to be optimally transmitted after optimization scales with its average contribution to the feedback signal. From Eq. 4.3 we see that the probability is proportional to $\left|E_{0}(\omega)\right|^{4}$.

Assuming that the spectral amplitude is only filtered by this weighting factor, the behaviour of the spectral amplitude would be given by $\left|E_{0}(\omega)\right|^{5}$ with a proportionality factor. The dash-dotted line in Fig. 4.4b indicates the pulse duration connected to this spectrum.

On closer inspection we have to consider that the frequency components do not independently contribute to the feedback signal but are connected by the Fourier transform and the second-order nonlinear process. We elaborated in the preceding section that the nonlinear feedback leads to a temporal focus, i.e., it forms a short pulse, which in inherently linked with the maintenance of a broad spectrum. This relation leads to a 'force' which counterbalances the spectral narrowing. Therefore the pulse duration connected to the spectrum $\propto\left|E_{0}(\omega)\right|^{5}$ sets the upper limit to $\Delta t_{\text {focus }}$. Equally, we can give a lower threshold for the concentration in time: The optimization reaches the highest peak amplitude at a single point in time $t_{0}$ when the set of phases $\phi_{a}$ exactly phase-conjugates the corresponding transmission coefficients 
$t_{a b}\left(t_{0}\right)$. This scenario is equivalent to a short-pulse WFS shaping experiment with a $\delta$ - gating pulse. For this case, the transmission probability for a narrow frequencyband is proportional to $\left|E_{0}(\omega)\right|$ and the resulting spectral amplitude is proportional to $\left|E_{0}(\omega)\right|^{2}$ (see chapter 3.A.2). The corresponding pulse duration is marked by the dashed line in Fig. 4.4b and we see that only very few simulations achieved pulse durations below this value.

Pulse durations below this threshold are physically possible, for example when a specific realization of the randomness sample realization allows to efficiently transmit a wider spectrum, but are unlikely. From a theoretical point of view, there is no direct limitation for the shortest pulse duration which can be achieved by a shortpulse WFS experiment: A spectral filtering step which selectively transmits the wings of the spectrum and extinguishes most of the central part of the spectral function will create a very broad spectrum and a very short pulse. However, this filtering goes at a cost of a tremendous loss of delivered intensity. Our optimization of the timeintegrated nonlinear intensity signal will therefore not converge to such a situation.

The exact focus pulse duration depends in a complex way on the starting conditions and the transmission coefficients, which will lead to a result between the two described situations. From Fig. 4.4b we note that the calculated thresholds agree with the limits of the simulated distribution.

\subsubsection{Pulse arrival time}

In this section we investigate the uniqueness of the spatiotemporal focus found by the optimization with a slow nonlinear detector by a simulated WFS experiment.

\section{Simulation}

We simulated two series of WFS experiments. For the first series, we generated one set of transmission coefficients $T(\omega)=\left[t_{1 b}(\omega), \ldots, t_{N b}(\omega)\right]$ which describes the propagation of the light from the $N$ SLMs segments to the detector at point $b$ for a single realization of a scattering medium. We then performed 500 WFS optimizations, each time starting with a different randomly generated set of SLM phases $\Phi=\left[\phi_{1}, \ldots, \phi_{N}\right]$. For the second series we equally performed 500 WFS optimizations, but each with a new realization of the medium with a new set of transmission coefficients $T(\omega)$. For both series and each optimization the closed-loop sequential optimization algorithm was used, which was iterated successively four times. Before and after each optimization the temporal position of the maximum amplitude of the transmitted field was determined.

\section{Results and discussion}

The result in Fig. 4.5a illustrates that a different configuration of the incident wavefront can lead to different optimized transmitted fields with deviating arrival times of the focus. The focus arrival time is spread over a wide time range both for the variation of the starting phase pattern (Fig. 4.5b) and the sample realization (Fig. 4.5c). Compared to the time distribution of highest speckle pulse before optimization, the 

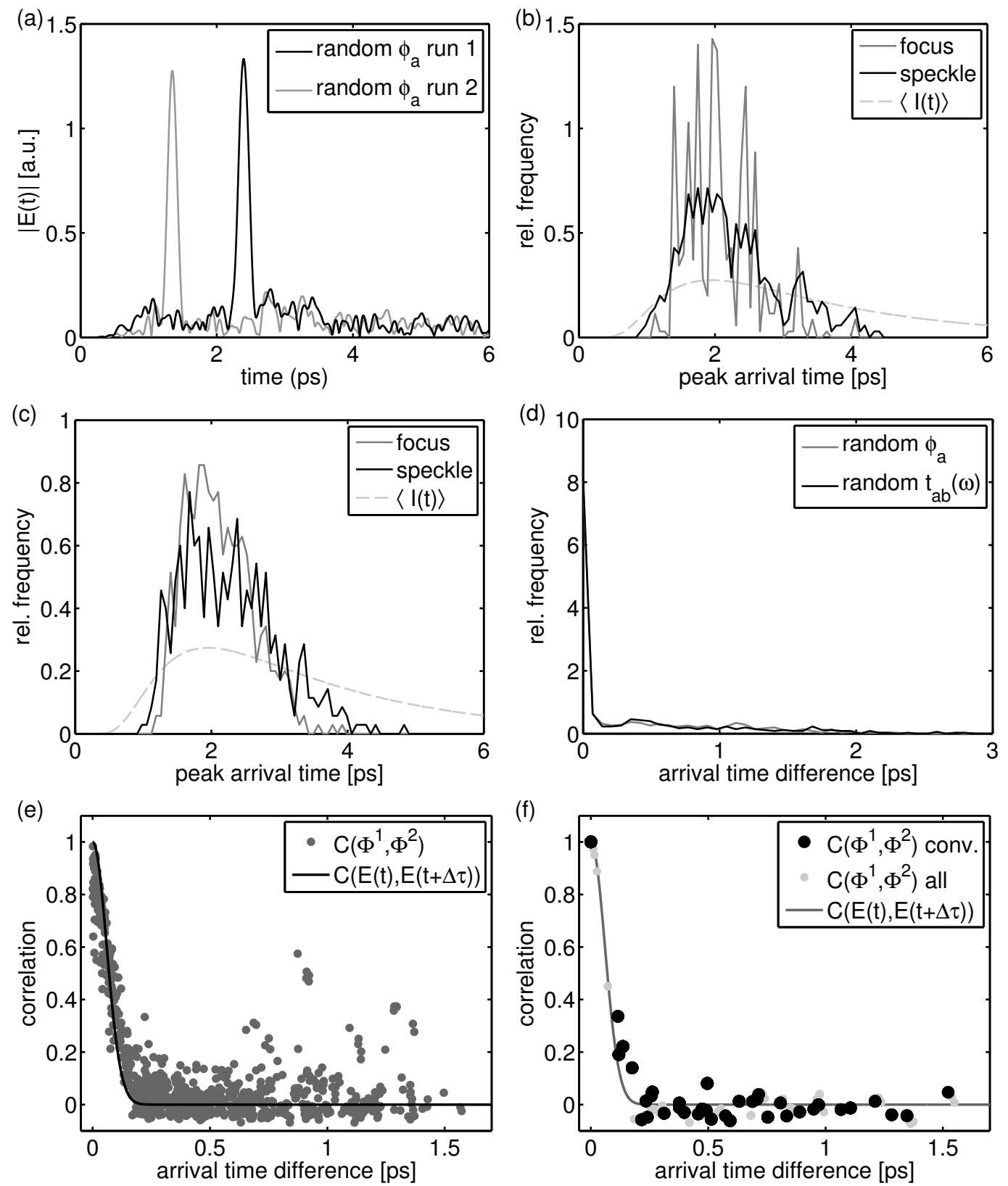

Figure 4.5: Results from a series of short-pulse WFS simulations with nonlinear feedback calculated by Eq. 4.2. (a) Temporal focus after two WFS optimizations with the same transmission coefficients $t_{a b}(\omega)$, but different random sets of starting phases $\phi_{a}$. (b) Histogram of the focus arrival time (grey line) and the maximum of the initial speckle pulse (black line) for 500 optimizations each with different starting phases $\phi_{a}$. The bin size was chosen equivalent to the input pulse duration $\Delta t$. The dashed line indicates ensemble averaged diffuse intensity. (c) Histogram of the focus arrival time for 500 simulated optimizations with different sets of transmission coefficients $t_{a b}(\omega)$. (d) Temporal shift between the maximum speckle amplitude before optimization and the focus arrival time. (e) Correlation between all pairs of phase vectors after the first iteration of the sequential algorithm of each individual optimization plotted over the interval between the corresponding peak amplitudes. (f) Correlation after the tenth iteration of the sequential algorithm. (Parameters for the simulations: $N=192$ segments, pulse duration $\Delta t=70 \mathrm{fs}$, center wavelength $\lambda=800 \mathrm{~nm}$, slab thickness $L=20 \mu \mathrm{m}$, diffusion constant $D=18.5 \mathrm{~m}^{\mathrm{s}} / \mathrm{s}$ ). 
focus arrival time is more concentrated around the peak of the average diffuse transmission $\langle I(t)\rangle$. From the difference of delay time of the highest speckle before optimization and the focal arrival time (Fig. 4.5d) we see that in about half of the investigated cases the temporal focus was formed close the highest initial speckle grain. Together, the last two observations can be interpreted with the picture that the probability of forming the focus at a given time increases the closer the time is to the peak of the average transmission. The width of the distributions is on the order of the decay time, which here for the given simulation parameters is $\tau_{d}=2 \mathrm{ps}$, calculated by Eq. 2.22 .

\section{Local maxima}

There is also a clear difference between the results of both simulations: The distribution for different sample realizations (Fig. 4.5c) is smoother than the distribution for different wavefronts (Fig. 4.5b) and we expect that the former will converge to a smooth curve without speckle with an increasing number of simulations. The latter distribution however shows pronounced, separated peaks, which indicate that the multi-dimensional optimization landscape for one sample realization has several local maxima.

In order to further investigate this point we repeated the simulation for one set of transmission coefficients and fifty different starting phase sets $\Phi^{i}$ with a very high number of iterations $\left(N_{i}=100\right)$. For each optimization we stored the phase set $\Phi^{i}$ after each iteration. We observed that forty out of the fifty optimizations had converged to the limit given by machine precision: The feedback signal only showed periodic fluctuations from iteration to iteration with relative changes below $1 \cdot 10^{-15}$, which is on the order the machine epsilon for double precision floating point formats. We made a pairwise comparison of all phase patterns after each iteration based on their correlation. We calculated the correlation between two sets of phases $\Phi^{1}=$ $\left[\phi_{1}^{1}, \ldots, \phi_{N}^{1}\right]$ and $\Phi^{2}=\left[\phi_{1}^{2}, \ldots, \phi_{N}^{2}\right]$ by

$$
C\left(\Phi^{1}, \Phi^{2}\right)=\frac{\left|\sum_{a=1}^{N} e^{i\left(\phi_{a}^{1}-\phi_{a}^{2}\right)}\right|-\sqrt{N}}{N-\sqrt{N}}
$$

We plotted the pairwise correlations for all phase sets over the interval between the arrival times of the corresponding peak amplitude after the first iteration in Fig. 4.5e and the last (100th) iteration Fig. 4.5f. In the second plot we additionally plotted the correlation calculated for only the subpopulation of converged optimizations (black dots).

The spread of arrival times of the converged solutions proves existence of different local maxima. We can distinguish two cases in Fig. 4.5f: In the first case two optimizations have converged to the same phase set and the same arrival time which means that the temporal focus is identical. In the second case the phase sets are uncorrelated, and the corresponding temporal foci are separated by a temporal gap which is larger than the correlation time. After one iteration all optimizations are far from convergence and this gap is not present yet. In the simulation here, the gap opens after four iterations (not shown), which means that at this stage all optimizations are in the attraction field of one of the local maxima towards which they will 
finally converge. The fact that some optimizations have not converged after even 100 iterations (grey dots in Fig. 4.5f) indicates that close to the local maxima gradients in the optimization landscape can be very shallow.

Here we identified nine local maxima with independent phase sets among the forty converged optimizations. From the ratio of the width of the distributions of the focus arrival time $\left(\approx \tau_{d}\right)$ over the distance between two local maxima $\left(\tau_{c}\right)$

$$
N_{t}=\frac{\tau_{d}}{\Delta \tau_{c}}
$$

we expect $N_{t}=14$ independent local maxima for the simulation parameters. Due to the relatively low number of repetitions of the simulation not all patterns were found.

\section{Finding the global maximum}

The sequential algorithm used in the simulation is a type of hill-climbing optimization which is not suitable to find the global maximum in a single execution. In the preceding paragraph we pointed out a crude method for using the sequential algorithm to find the global optimum: If the number of local maxima can be approximated, the optimization simply needs to be repeated with different starting phases until the same number of uncorrelated phase patterns are found.

For a more systematic approach the SHG optimization needs to be combined with a time-resolved feedback such as used in chapter 3. The light is in the first step temporally focused with the time-resolved feedback. Starting from this configuration, the SHG is optimized and will converge to the closest local maximum. To evaluate all local maxima, the simulation or the experiment is repeated for different delay times of the time-resolved feedback signal over the relevant time span.

The questions is whether alternative algorithms can find the global optimum in a more efficient way without additional assistance of a time-resolved feedback. The algorithm of choice needs to cope with the following optimization problem: There are several local maxima in a high-dimensional optimization landscape. As the corresponding phase-patterns are uncorrelated, the distance between local maxima on this landscape is large and gradients on the connecting surface are steep. The values of different local maxima are very similar, such that maxima can only be evaluated close to the point of convergence. We furthermore assume that the final value of a local maximum is fairly independent of the gradients which lead to the maximum: There is only a very weak correlation between the initial amplitude of a high starting speckle grain and the final value of the connected local maximum.

Frequently-used non-hill-climber algorithms to solve complex optimization tasks are the simulated annealing algorithms and genetic algorithms. Neither of the approaches on their own appears very beneficial for the given problem: Since the local maxima are very distant on the optimization landscape, we expect that a simulated annealing-type will behave similarly to the sequential algorithm. Because the phase patterns of separated local maxima are uncorrelated, the genetic algorithm is neither appealing: The key principle of the genetic algorithm, which is the testing of combinations of 'strong genes', only helps for the convergence to one local maximum.

We envision that a combination of different approaches in a multi-stage algorithm will be most suitable. In the initial step a random algorithm or a genetic-type al- 
gorithm generates new phase sets. Above a certain threshold of the feedback signal the optimization is continued by a hill-climber algorithm to quickly approach a local maximum. Any phase set is rejected when the correlation with previously found phase sets exceeds a certain threshold. Close to the point of convergence a simulated annealing-type algorithm takes over when the hill-climber algorithm stalls.

\subsubsection{Conclusions and outlook}

We demonstrated based on simulations that short-pulse WFS leads to temporal focusing with a slow nonlinear detector. The resulting focus pulse duration is comparable to the result from short-pulse WFS with time-resolved feedback achieved with a much simpler experimental apparatus. The 'price to pay' is a loose of control on the arrival time of the focused pulsed $\tau_{\text {focus }}$ and a certain spread of the obtained pulse duration $\Delta t_{\text {focus. }}$. Regarding this aspect the simulations prove very helpful: The number of simulations can easily exceed the possible number of experiments in the lab. This gives us valuable insights in the distribution of $\tau_{\text {focus }}$ and $\Delta t_{\text {focus }}$ which are otherwise more difficult to obtain. We did not explicitly investigate the spatial aspect of the focusing. Because a spatiotemporal speckle pattern has a limited spatial correlation length, we can expect that the temporal focus is also spatially confined even for a detector whose size spans several speckle spots. The full power of the approach will be exploited in the following chapters: if a probe particle at the target has a nonlinear radiative response, the point of the nonlinear process and the final detection and conversion to an electronic signal can be spatially separated. 


\section{Spatiotemporal focusing in turbid by wavefront shaping with nonlinear feedback}

We present an experimental study of short-pulse wavefront shaping (WFS) with a slow nonlinear local detector: We demonstrate the spatiotemporal focusing of light on single nanocrystals embedded in a strongly scattering medium, using second harmonic generation (SHG) inside the target nanocrystals as the feedback signal for the WFS algorithm. The nanocrystals are placed at the back interface of the scattering medium, such that the enhancement of the SHG signal from single particles can be accurately monitored by a camera. We successfully develop a model both for the achieved pulse duration as well as the observed enhancement of the feedback signal. The results lay the foundations for a wide range of novel experiments, and in particular for our own study presented in chapter 6 .

\subsection{Introduction}

Achieving optimal control of light propagation in any type of complex scattering medium is highly desirable for applications in imaging, micro- and nanomanipulation, coherent control and spectroscopy. However, in numerous systems of interest of current research, for example in thick biological specimens, random multiple scattering is a major obstruction for the performance of conventional optical techniques.

Recently, Vellekoop and Mosk demonstrated that random multiple light scattering can be exploited to increase rather than hamper the control of light propagation [13].

The content of this chapter has been published as: J. Aulbach, B. Gjonaj, P. Johnson, and A. Lagendijk, Opt. Express 20, 29237 (2012). 
The approach, named 'wavefront shaping' (WFS) is based on spatial modulation of the complex amplitude of the light incident on the medium. With an adaptive algorithm the optimal wavefront is determined, which matches the medium such that coupling of light to a desired output mode is maximized, e.g., a focal spot behind the medium. In this way the scattering medium can be turned into a lens to focus light for trapping nanoparticles or for high-resolution imaging as recent applications have demonstrated $[24,121]$. Requiring only an intensity measurement as feedback signal makes the approach powerful and versatile, enabling focusing on fluorescent beads embedded inside a complex medium [14] without the need for phase-sensitive measurement techniques.

For acoustic waves and for microwaves, related experiments have demonstrated broadband focusing in complex scattering media using a time reversal mirror [39]. Based on this technique, Lerosey et al. [42] focused electromagnetic waves onto a small antenna inside a dense assembly of resonant scatterers with precision far below the wavelength of the radiation. However, for optics, the realization of an optical time reversal mirror remains challenging, requiring both an accurate broadband measurement and the synthesis of a complex electric field. The existing optical analog, phase conjugation, is effectively limited to monochromatic light [26, 27, 134].

While the initial optics experiments were performed with monochromatic light, WFS was recently extended to the broadband regime. Spatial wavefront shaping in combination with a scattering medium allows both spatial and temporal control of the transmitted scattered light using optical gating as feedback [135] or pulse compression through the medium using two-photon fluorescence [109].

Simultaneous spatial and temporal focusing on nanoparticles embedded inside a strongly scattering medium has not been demonstrated yet. A promising approach to achieve this goal is a wavefront shaping experiment with a feedback signal from nanoparticles with a nonlinear optical response which are to be embedded inside the medium [136]. Possible candidates for these probe particles are fluorescent dyes or quantum dots as commonly used for two-photon microscopy [3]. Especially interesting are nanocrystals from wide bandgap materials with a high efficiency for second harmonic generation (SHG) [137-139]: Providing a stable and coherent SHG signal, and flexibility for use in a wide spectral range, this class of particles recently gained considerable attention as markers for novel microscopy techniques [140] and makes them ideal probes for WFS experiments.

Here we experimentally demonstrate spatiotemporal focusing on single nanocrystals embedded inside a strongly scattering medium. Our approach is based on wavefront shaping of short pulses, using second harmonic generation inside the target nanocrystals as feedback signal. We develop a model both for the achieved pulse duration at the particle position as well as the observed enhancement of the feedback signal which is in good agreement with the experiment. Our approach has implications for applications in which for control of light propagation in complex media is required and provides a powerful tool to study light propagation in the presence of strong multiple scattering.

This chapter is structured as follows. At first we describe the experiment in Sec. 5.2. It is followed by a detailed and extensive theory section (Sec. 5.3) before we provide the experimental results in Sec. 5.4. 


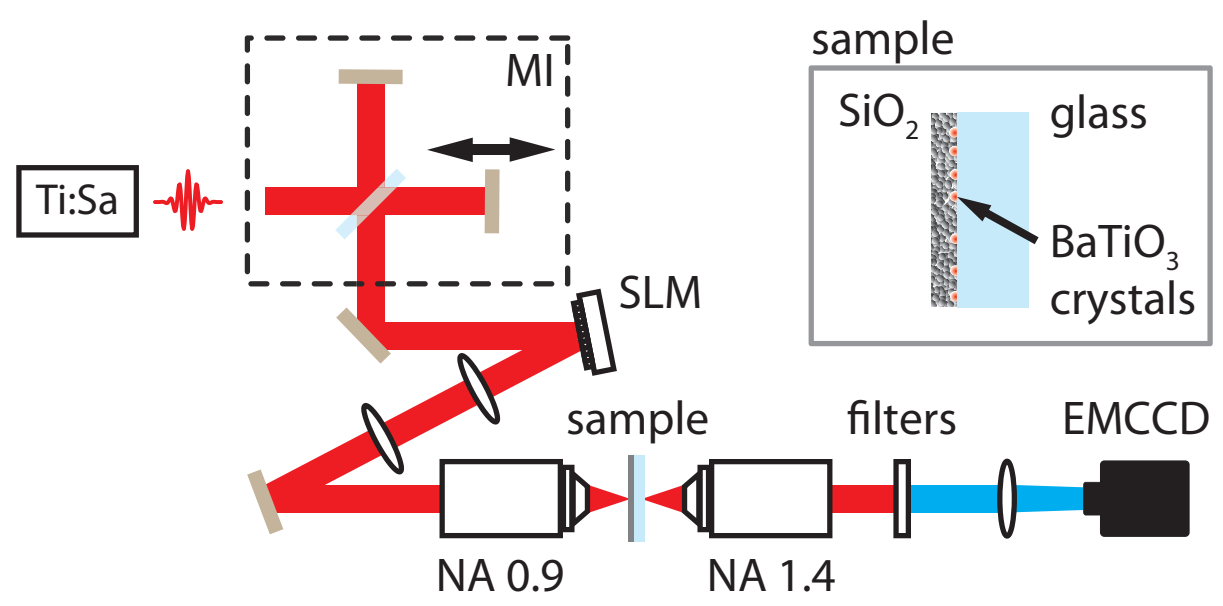

Figure 5.1: Schematic illustration of the experiment. (Ti:Sa) Ti:sapphire laser, (MI) Michelson-type interferometer, (SLM) spatial light modulator, (NA) numerical aperture, (EMCCD) electron-multiplying CCD camera

\subsection{Experiment}

The general idea of the experiment can be described as follows. The pulse front impinges on the surface of a thick multiple scattering medium. The light reaching positions inside the medium at depths larger than a few mean free paths has been multiply scattered such that the spatial structure of the incident beam is lost and temporally the pulses are elongated to random speckle pulses. It is our goal to focus the light spatiotemporally on a single point in the medium at a single moment in time. We apply spatial wavefront shaping, using second-harmonic active nanocrystals to deliver the feedback signal. The particles act as a local probe of the light intensity, converting the present light from the fundamental wavelength to the second-harmonic wavelength, which can be readily detected separately from the fundamental light. Since the particle is a local probe, we can expect that the algorithm will locally maximize the light intensity. The local second-harmonic generation not only acts as a spatial filter, but is also biased towards shorter pulse durations, leading to additional temporal focusing.

The experiment is illustrated in Fig. 5.1. The light from the Ti:sapphire laser (repetition rate $80 \mathrm{MHz}$, output power $1.2 \mathrm{~W}$, center wavelength $841 \mathrm{~nm}$, bandwidth $19 \mathrm{~nm}$, pulse duration $67 \mathrm{fs}$ ) is sent through a Michelson-type interferometer. One interferometer arm is of fixed length, the other has a variable delay (Newport XMS100). Subsequently the beam is expanded 6:1 (not shown) to fill the surface of a twodimensional phase-only spatial light modulator (SLM, Hamamatsu X10468). The SLM pixels are grouped into $N$ independent segments each of which induces a controllable phase shift. Two lenses project the SLM surface onto the back focal plane of an air-immersion objective (numerical aperture (NA) 0.9, x100). The focal plane of the objective is a conjugate plane of the SLM surface with a total magnification of $1 / 300$. The sample is placed in the focal plane of the objective. The transmitted light through the sample is collected by an oil-immersion objective (magnification 100x, NA 1.4). After filtering (short-pass filter $675 \mathrm{~nm}$ and bandpass filter $420 \mathrm{~nm}$ ), only the generated second harmonic is imaged by an electron multiplying CCD camera 
Spatiotemporal focusing in turbid media by wavefront shaping with nonlinear feedback

(Andor DV885, frame rate $0.25-2 \mathrm{~Hz}$, total magnification 93.5/1). Optionally the laser light can be directly coupled into a Fourier transform spectrometer instead of entering the setup (not shown).

The sample is depicted in the inset of Fig. 5.1 and consists of a disordered layer of silica beads which covers sparsely distributed second-harmonic active nanocrystals on a standard glass coverslip (thickness $0.17 \mathrm{~mm}$ ). The nanocrystals consist of barium titanate $\left(\mathrm{BaTiO}_{3}\right)$ with a tetragonal crystal structure and an average diameter of $200 \mathrm{~nm}$. Prepared using the method described in [141], about $90 \%$ of the nanocrystals are not clustered and are well-separated from other particles on the glass coverslip, which we confirmed by imaging a sample of nanocrystals without the scattering medium. The layer of silica spheres (diameter $400 \mathrm{~nm}$ to $500 \mathrm{~nm}$ ) has been applied on top of the nanocrystals by spray-coating a colloidal dispersion in ethanol, which after drying forms a rough surface with a varying layer thickness between $L=25 \mu \mathrm{m}$ and $L=50 \mu \mathrm{m}$. The mean free path of this medium $(l=3.5 \mu \mathrm{m})$ has been determined by measuring the enhanced backscattering cone. During the application of the silica dispersion with the spray-coating technique most of the barium titanate nanocrystals are detached from the glass coverslip, but remain in the vicinity of the substrate interface within the first few layers of silica beads (rather than diffusing deep into the silica layer) such that they can be identified as individual sources of second harmonic generation.

For every wavefront shaping experiment presented in this chapter we use the following procedure. At first, we take the average image of a large number of images of random wavefronts (with a segmentation of SLM into $N=768$ segments), which serves as a reference measurement for the obtained enhancement of the feedback signal. Then a flat phase pattern is applied to the SLM, and we perform a spatially resolved second-harmonic autocorrelation measurement [108] by recording images while the movable arm of the Michelson interferometer is scanned in discrete steps. The step resolution of the delay stage $(10 \mathrm{~nm})$ allows us to record the full interferometric autocorrelation trace. To speed up the data acquisition we measure only parts of this trace, applying a two-step algorithm. After a large step $(2.5 \mu \mathrm{m}$ or $5 \mu \mathrm{m})$, the stage samples one optical cycle $(841 \mathrm{~nm})$ in eight steps, from which the cycle-averaged intensity autocorrelation is calculated. Then the wavefront shaping algorithm is started. We use a sequential algorithm; it addresses segment by segment one by one, scanning the phase from $\varphi=0$ to $\varphi=2 \pi$ in $N_{\varphi}=8$ steps. For each step we record the time-integrated second-harmonic radiation from a single nanocrystal retrieved from the camera image as feedback signal. The phase value which maximizes the feedback signal is determined by fitting the measured behaviour of the feedback signal vs. phase with a cosine function. We perform several consecutive sequences over all segments of the SLM, starting with a low segmentation of the SLM (48 segments) which is increased twice after the first sequences (to 192 and 768 respectively), always starting the new sequence with the phase pattern obtained from the previous one. After the algorithm has finished, another autocorrelation measurement is performed. 


\subsection{Theory}

\subsubsection{Intensity-intensity autocorrelation of speckle pulses}

Our experimental setup enables us to measure the second-harmonic autocorrelation $A C(\tau)$ [108] spatially resolved at the particle position as a function of delay $\tau$ introduced in the Michelson interferometer. For the discussion, we consider ensemble averaged intensity autocorrelation with background, which are

$$
\langle A C(\tau)\rangle=\int d t\left\langle I^{2}(t)\right\rangle+2 \int d t\langle I(t) I(t+\tau)\rangle .
$$

where $I(t)$ denotes the intensity at the fundamental frequency and the brackets denote the ensemble average over all realizations of the scattering medium. For short pulses the observed contrast ratio between the maximum and the background is $\mathrm{AC}(\tau=0): \mathrm{AC}(\tau \rightarrow \infty)=3: 1$, regardless of the exact temporal shape of the intensity $I(t)$.

In the diffuse regime, the average time-dependent transmission $\left\langle I_{\mathrm{d}}(t)\right\rangle$ through a disordered slab shows an exponential decay with the diffuse decay time $\tau_{\mathrm{d}}$ as characteristic time scale (see Eq. 2.22). Neglecting boundary effects, it is related to the thickness of the medium by $\tau_{\mathrm{d}}=L^{2} / \pi^{2} D$, where $D$ is the diffusion constant. An exact expression for the temporal behaviour of $\left\langle I_{\mathrm{d}}(t)\right\rangle$ for the diffuse transmission through a slab is given in [99]. For a single experimental realization, the transmitted intensity $I_{\mathrm{d}}(t)$ is dominated by temporal speckle. Characteristically for such a speckle pulse, it has a limited temporal coherence time $\tau_{\mathrm{c}}$, which is given by the inverse bandwidth of its intensity spectrum. Therefore, the speckle pulses can be described as a random sequence of short pulses, each approximately of the duration $\tau_{\mathrm{c}}$, distributed randomly in a time window of the diffuse decay time of the medium $\tau_{\mathrm{d}}$.

The particular shape of the autocorrelation function of a speckle pulse, observed after transmission through a slab of turbid material, can be described as follows. The maximum of the autocorrelation for $\tau=0$ decays on the time scale $\tau_{\mathrm{c}}$, since for larger delay times the intensities $I_{\mathrm{d}}(t)$ and $I_{\mathrm{d}}(t+\tau)$ within a speckle pulse are uncorrelated random variables given by the Gaussian statistics of the transmission process. However, for thick scattering media with $\tau_{\mathrm{d}} \gg \tau_{\mathrm{c}}$, the average intensity $\left\langle I_{\mathrm{d}}(t)\right\rangle$ is approximately constant on timescales of $\tau_{\mathrm{c}}$. For $\tau>\tau_{\mathrm{c}}$ the value of the integral on the right-hand side can be approximated by separating the product into $\left\langle I_{\mathrm{d}}(t)\right\rangle\left\langle I_{\mathrm{d}}(t+\tau)\right\rangle$. Therefore the correlation term does not directly decay to the background level, but shows an intermediate regime resulting from the incoherent part of the speckle pulse. Since for Gaussian statistics $\left\langle I_{\mathrm{d}}(t)\right\rangle^{2}=0.5\left\langle I_{\mathrm{d}}(t)^{2}\right\rangle$, the coherent peak is separately visible on top of the diffuse peak with a contrast ratio 3:2:1 over the background. Altogether, we can model the normalized autocorrelation of the speckle pulses by

$$
\frac{\langle A C(\tau)\rangle}{\langle A C(\tau=0)\rangle}=1+\frac{\int d t\left\langle I_{\mathrm{d}}(t)\right\rangle\left\langle I_{\mathrm{d}}(t+\tau)\right\rangle}{\int d t\left\langle I_{\mathrm{d}}(t)\right\rangle^{2}}+\frac{\int d t I_{0}(t) I_{0}(t+\tau)}{\int d t\left(I_{0}(t)\right)^{2}} .
$$

The second term on the right-hand side of Eq. (5.2) is the normalized contribution of the average diffuse transmission $\left\langle I_{\mathrm{d}}(t)\right\rangle$, the third term is the normalized contribution from the non-scattered bandwidth limited short pulse $I_{0}(t)$ provided by the laser. 
Spatiotemporal focusing in turbid media by wavefront shaping with nonlinear feedback

\subsubsection{Enhancement of the time-integrated second harmonic intensity}

\section{Enhancement vs. number of segments}

The wavefront shaping algorithm maximizes the time-integrated second-harmonic intensity generated at the particle position. In the following, we derive an expression for the average enhancement of this feedback signal which is achieved after wavefront shaping.

First, we derive the enhancement expected from a hypothetical monochromatic experiment with the second-harmonic intensity as feedback. The polarization at the second harmonic frequency $P_{2 \omega}$ at the particle positioned at point $b$ can be calculated by

$$
P_{2 \omega}=\chi_{(2)} E_{b}^{2}=\chi_{(2)} \sum_{a=1}^{N} t_{a b} E_{a} \sum_{a^{\prime}=1}^{N} t_{a^{\prime} b} E_{a^{\prime}} .
$$

The complex transmission coefficients $t_{a b}$ describe the propagation of the electric field $E$ from SLM segment $a$ to the particle position $b$. Here, we have made a number of simplifications; we neglect the polarization of the light, as well as the tensor character of the second-order susceptibility $\chi_{(2)}$ of the nanocrystals. Furthermore we assume that the volume speckle inside the medium is larger than the particle size, and the particle radiates like a dipole at the second harmonic frequency. The radiated second harmonic power can be calculated by [142]

$$
W_{2 \omega}=\frac{c k^{4} V^{2}}{12 \pi \epsilon_{0}}\left|P_{2 \omega}\right|^{2}
$$

with the speed of light $c$, the particle volume $V$ and the vacuum permittivity $\epsilon_{0}$. In a wavefront shaping experiment, the electric field on each SLM segment $a, E_{a}=A_{a} e^{\varphi_{a}}$, is adapted with the aim that all contributions are in phase at the particle position, e.g., $\varphi_{a}=-\arg \left(t_{a b}\right)$ for all segments $a$. In our experiment, the phase-only SLM does not significantly modify the amplitudes $A_{a}$. As described above, we use a sequential algorithm for the WFS experiment; it addresses all segments one by one, scanning the phase term $\varphi$ from 0 to $2 \pi$ in discrete steps. Given that the segment of interest contributes to the field as $E_{1} e^{i \varphi} \equiv t_{a b} A_{a} e^{i \varphi}$ at the point of interest, while the sum of unmodified segments contributes by $E_{2} \equiv \sum_{a^{\prime}, a^{\prime} \neq a}^{N} t_{a^{\prime} b} E_{a^{\prime}}$, the second harmonic power during the phase scan is given by

$$
\begin{gathered}
W_{2 \omega}(\varphi) \propto \quad\left|E_{1}^{2}\right|^{2}+\left|E_{2}^{2}\right|^{2}+4\left|E_{1}\right|^{2}\left|E_{2}\right|^{2}+\Re\left\{4 E_{1} E_{2}^{*}\left|E_{1}\right|^{2} e^{i \varphi}\right\}+ \\
\left.\Re\left\{2 E_{1}^{2}\left(E_{2}^{*}\right)^{2} e^{2 i \varphi}\right\}+\Re\left\{4 E_{1} E_{2}^{*}\left|E_{2}\right|^{2} e^{i \varphi}\right\}\right)
\end{gathered}
$$

Typically $\left|E_{2}\right| \gg\left|E_{1}\right|$ holds, such that the last term on the right hand side dominates the varying terms, and the behaviour of $W_{2 \omega}(\varphi)$ is well described by a cosine function with a constant offset.

By evaluating the right hand side of Eq. (5.3), the ensemble averaged second harmonic power can be calculated,

$$
\left\langle W_{2 \omega}\right\rangle=2 N(N-1)\left\langle\left|t_{a b}\right|^{2}\right\rangle^{2}+N\left\langle\left|t_{a b}\right|^{4}\right\rangle .
$$


For simplicity, we dropped the constants from Eq. (5.3) and Eq. (5.4) and set the field amplitudes to one.

The average resulting second harmonic intensity after WFS is equal to

$$
\begin{aligned}
\left\langle W_{2 \omega}\right\rangle_{\mathrm{wfs}}= & \left\langle W_{2 \omega}\right\rangle \\
& +N(N-1)\left\langle\left|t_{a b}\right|^{2}\right\rangle^{2} \\
& +6 N(N-1)(N-2)\left\langle\left|t_{a b}\right|^{2}\right\rangle\left\langle\left|t_{a b}\right|\right\rangle^{2} \\
& +4 N(N-1)\left\langle\left|t_{a b}\right|^{3}\right\rangle\left\langle\left|t_{a b}\right|\right\rangle \\
& +N(N-1)(N-2)(N-3)\left\langle\left|t_{a b}\right|\right\rangle^{4} .
\end{aligned}
$$

Taking only the highest order terms in $N$ into account, the observed enhancement is approximately given by

$$
\eta_{\mathrm{cw}} \equiv \frac{\left\langle W_{2 \omega}\right\rangle_{\mathrm{wfs}}}{\left\langle W_{2 \omega}\right\rangle} \approx 0.5\left(\frac{\langle|A|\rangle^{2}}{\left\langle|A|^{2}\right\rangle}\right)^{2}\left(\frac{\left\langle\left|t_{a b}\right|\right\rangle^{2}}{\left\langle\left|t_{a b}\right|^{2}\right\rangle}\right)^{2} N^{2},
$$

where we reintroduced the distribution of the contributing field amplitudes A from each of the $\mathrm{N}$ segments.

\section{Enhancement for speckle pulses}

In our experiment, the measured second-harmonic signal is not generated from a continuous wave, but from speckle pulses at the particle position. Effectively the wavefront shaping optimization affects the original speckle pulse only within a time window of duration of the coherence time $\tau_{\mathrm{c}}$ around the point in time at which the optimized pulse is formed. Within this temporal window, the enhancement is given by Eq. (5.8). At earlier or later moments, the speckle pulse will be modified in a random fashion, but on average it will be unaffected by the optimization.

Since this non-optimized part will always contribute to the time-integrated secondharmonic intensity (i.e., the energy), the enhancement of the energy $\eta_{\text {pulsed }}$ will be lower compared to the hypothetical continuous-wave case. We define the reduction factor $c_{\tau}$ by

$$
c_{\tau} \equiv \frac{\eta_{\text {pulsed }}}{\eta_{\mathrm{cw}}}
$$

In a first approximation, we expect the enhancement to be lowered by the inverse number of independent temporal speckle grains within a speckle pulse, which is approximately given by the ratio $c_{\tau} \approx \tau_{\mathrm{c}} / \tau_{\mathrm{d}}$. As the diffuse decay time is proportional to the thickness squared (see Sec. 5.1), $c_{\tau}$ approximately scales with $L^{-2}$. However, the ratio $\tau_{\mathrm{c}} / \tau_{\mathrm{d}}$ does not exactly reflect the temporal distribution of the second harmonic intensity. For a known average intensity of the fundamental light at the particle position $\langle I(t)\rangle$ we can calculate the correction factor $c_{\tau}$ more precisely. The average generated second harmonic intensity is consequently proportional to $\langle I(t)\rangle^{2}$. The reduction factor $c_{\tau}$ for the enhancement compared to the monochromatic case is calculated from the ratio of the energy of the generated second harmonic in a time-window $\tau_{\mathrm{c}}$ around the time $t_{\max }$ of the maximum of $\langle I(t)\rangle^{2}$ and the total second harmonic energy,

$$
c_{\tau}=\frac{\int_{t_{\max }-\tau_{\mathrm{c}} / 2}^{t_{\max }+\tau_{2}} d t\langle I(t)\rangle^{2}}{\int_{-\infty}^{+\infty} d t\langle I(t)\rangle^{2}}
$$


Spatiotemporal focusing in turbid media by wavefront shaping with nonlinear feedback

\section{Susceptibility tensor}

In our model we treat the nanocrystal as a single radiating dipole. For a detailed analysis, the light polarization and the second-order susceptibility tensor needs to be considered. The second-order polarization at the second harmonic frequency can be calculated by the matrix equation $[137,143]$

$$
\mathbf{P}_{2 \omega}=\left[\begin{array}{cccccc}
0 & 0 & 0 & 0 & d_{15} & 0 \\
0 & 0 & 0 & d_{15} & 0 & 0 \\
d_{31} & d_{31} & d_{33} & 0 & 0 & 0
\end{array}\right]\left(\begin{array}{c}
E_{\mathrm{c} x}^{2} \\
E_{\mathrm{c} y}^{2} \\
E_{\mathrm{c} z}^{2} \\
2 E_{\mathrm{c} y} E_{\mathrm{c} z} \\
2 E_{\mathrm{c} x} E_{\mathrm{c} z} \\
2 E_{\mathrm{c} x} E_{\mathrm{c} y}
\end{array}\right)
$$

where the $E_{\mathrm{c} i}$ are the orthogonal components of the electric field along the three axis in the crystal frame and the $d_{i j}$ are the second-order susceptibilities of the bulk $\mathrm{BaTiO}_{3}$ crystal. The considered values are $d_{15}=-41 \cdot 10^{-9} \mathrm{esu}, d_{31}=-43 \cdot 10^{-9} \mathrm{esu}$ and $d_{33}=-16 \cdot 10^{-9} \mathrm{esu}$ [143]. Note that the second-harmonic response is independent of a rotation around the z-axis. The position of the latter in the lab frame is sufficient to describe the second-harmonic response of the nanocrystals, assuming that they are spherical. From Eq. (5.11) we can see that all components of the vector on the right hand side of Eq. (5.11) with a non-zero second-harmonic response $\left(E_{\mathrm{c} x}^{2}, E_{\mathrm{c} y}^{2}, E_{\mathrm{c} z}^{2}, 2 E_{\mathrm{c} y} E_{\mathrm{c} z}, 2 E_{\mathrm{c} x} E_{\mathrm{c} z}\right)$ compete for optimization during the wavefront shaping optimization. We assume that the transmission coefficients connecting the SLM segments with each of the crystal axis are independent.

For an illustrative purpose we first analytically analyze the case in which the algorithm optimizes all contributions in the $E_{\mathrm{c} x}$ component. Assuming that the detection efficiency is equal for the second harmonic radiation from all crystal axis, and that before optimization the ensemble averaged fields on the three crystal axis $\left\langle\left|E_{\mathrm{c} i}\right|\right\rangle$ are equal, the average generated second harmonic power is proportional to $\left(2 d_{31}^{2}+d_{33}^{2}+4 d_{15}^{2}\right)$. Since only the $E_{\mathrm{c} x}$ components, which are generating second harmonic proportional to $\alpha_{31}^{2}$, are enhanced (with a factor given by the formulas above), the total enhancement is modified by the factor $c_{\alpha}=d_{31}^{2} /\left(2 d_{31}^{2}+d_{33}^{2}+4 d_{15}^{2}\right) \approx 0.17$. We thereby assume that the optimized component is significantly larger than $E_{\mathrm{c} z}$ after optimization, such that the cross-terms $E_{\mathrm{c} y} E_{\mathrm{c} z}$ and $E_{\mathrm{c} x} E_{\mathrm{c} z}$ can be neglected.

In order to obtain a correction factor close to our experimental situation, we performed numerical simulations of the WFS experiment. For each run of the simulation, we generate a set of random transmission coefficients connecting each of the SLM segments with the three orthogonal field contributions at the crystal position, assuming that the average fields $\left\langle\left|E_{\mathrm{c} i}\right|\right\rangle,\{i=x, y, z\}$ are equal. To calculate the feedback signal, we first apply Eq. (5.11) to calculate the second-order polarization in the crystal frame. Secondly, the polarization vector is calculated in the lab frame, depending on the orientation of the nanocrystal. Finally, we calculate the second-harmonic intensity as it is collected by a high-NA $(\mathrm{NA}=1.4)$ objective corresponding to our experimental parameters. We apply the sequential optimization algorithm, such as applied in our experiment. As a result, we observe that the algorithm in generally optimizes both the $E_{\mathrm{c} z}$ and the $E_{\mathrm{cx}}$ or $E_{\mathrm{cy}}$ component, with a ratio which varies slightly with crystal orientation. Averaged over all crystal positions, we find that the 
enhancement of the feedback is modified by the factor $c_{\alpha}=0.28 \pm 0.04$ compared to the scalar model (Eq. (5.8)). Due to the large collection angle, the light radiated from all crystal axis is approximately collected with equal efficiency. The dependence of the factor $c_{\alpha}$ on the crystal orientation is superseded by variations caused by random variations of the transmission coefficients.

\section{Correction for tight focus}

In the introduction of our model we assumed nanocrystals which are much smaller than the focal volume after the optimization. However, the focus is created in a high index medium $(\mathrm{n}=2.3)$ and the medium effectively acts as a lens with a large acceptance angle of about $90^{\circ}$. To a first approximation, the optimization will minimize the focal volume, since it leads to the highest peak intensity and consequently the highest efficiency for the SHG process. Therefore, for a correct description of our experiment we have to consider that the focal volume after WFS will be smaller than the particle volume, whereas before WFS the whole particle volume contributes equally to the average feedback signal. However, the exact shape and polarization state of the tight focus which is formed during a WFS experiment depends on the particle size and shape, the crystal orientation and the specific realization of the photonic environment. Modeling the exact shape of the optimal focus and consequently the generated second harmonic signal in a barium titanate nanocrystal with a size beyond the electrostatic limit is far from trivial, considering that 'simpler' systems with centrosymmetric materials already require an extensive theoretical treatment [144].

In the following we assess the problem in a simplified treatment. Similar to calculations by van Putten et al. for a linear feedback [145], we calculate the correction factor of the enhancement for second-harmonic generation by

$$
c_{R} \equiv \frac{1}{V} \int_{0}^{R} \int_{0}^{\pi} \int_{0}^{2 \pi} \frac{I_{2 \omega}(\phi, \theta, r)}{I_{2 \omega, \text { peak }}} r^{2} \sin \theta \mathrm{d} r \mathrm{~d} \phi \mathrm{d} \theta,
$$

where $V$ is the particle volume of the nanocrystal, and the fraction is the positiondependent intensity $I_{2 \omega}(\phi, \theta, r)$ of the second harmonic radiation integrated over the three crystal axis, normalized to its peak intensity $I_{2 \omega \text {,peak }}$ at the center of the focus. To assess the focal volume quantitatively, we assume that the focus on the nanocrystal has the same profile as a focus created with a high NA lens with and acceptance angle of $90^{\circ}$. From [137] we can conclude that the crystal orientation determines whether linearly or circularly polarized light is more efficient for the SHG process. We assume that the WFS process always converges to the optimal polarization state. We calculate the field distribution of the fundamental radiation at the focus according to [146]. Using the field distribution, we obtain $I_{2 \omega}(\phi, \theta, r)$ using the susceptibility tensor given in Eq. (5.11). We perform this calculation for a sufficient number of polarization states between linearly and circularly polarized light. We calculate $c_{R}$, averaged over all orientations of the crystal c-axis. For each angle we thereby consider only the respective polarization state which maximizes the SHG process. We used the parameters of the particle radius $R=100 \mathrm{~nm}$, and the refractive index $\mathrm{n}=2.3$. 
Spatiotemporal focusing in turbid media by wavefront shaping with nonlinear feedback

We find $c_{R}=0.57 \pm 0.05$. We observe that $c_{R}$ is approximately proportional to the radius in the considered size regime. For the crystals used in our experiment, we estimate a size polydispersity of $25 \%$, which will result in equivalent variations of $c_{R}$.

Note that the simplification of our approach in Eq. (5.12) is twofold. Firstly, photonic effects from the spherical shape are neglected. Secondly, the second harmonic intensities within the volume are integrated, and not the electric fields, neglecting interference between different dipole radiating at the second harmonic frequency. We assume that both effects will affect both the numerator of Eq. (5.12) (optimized focus) and the denominator (reference signal from the average wave field) in the same fashion and therefore tend to level out. Given that the correction factor calculated with our simplified model is rather moderate with 0.57 (compared to the value 1 for a sphere smaller than the focal volume), we do not expect a drastic deviation of the correction factor if these effect would be taken into account.

\section{Noise}

The presence of noise on the measurement of the feedback will reduce the observed enhancement. For segments whose contribution to the feedback is on the order or below the noise level, the correct phase value will not be found. Taking this effect into account, we extend Eq. (5.8) to

$$
\eta_{\mathrm{cw}} \approx 0.5\left(\frac{\left\langle\left|A_{a} t_{a b}\right| \cdot \gamma\left(\left|A_{a} t_{a b}\right|, \sigma_{a}, N_{\varphi}\right)\right\rangle^{2}}{\left\langle\left|A_{a} t_{a b}\right|^{2}\right\rangle}\right)^{2} N^{2} .
$$

The function $\gamma\left(\left|A t_{a b}\right|, \sigma, N_{\varphi}\right)$ is the correlation between the optimal phase $\phi_{a}=$ $-\arg \left(t_{a b}\right)$ and the phase in the presence of noise $\phi_{\sigma}$ for a given noise level $\sigma_{a}$ and the magnitude of the contribution given by $\left|A t_{a b}\right|$.

In the following we describe how $\gamma\left(\left|A t_{a b}\right|, \sigma_{a}, N_{\varphi}\right)$ is calculated. The phase which is obtained from the fit of the feedback signal with a cosine function (see sections 5.2 and 5.3.2) is equivalent to the phase of the first non-zero frequency component of a discrete $N_{\varphi}$-point Fourier transform of the feedback scan. Component $k$ of the Fourier transform is given by

$$
\mathrm{FT}_{\mathrm{k}, \mathrm{a}} \equiv \sum_{n=1}^{N_{\varphi}}\left\{I_{2 \omega, a}\left(\varphi_{n}\right)\right\} e^{-i 2 \pi \frac{k}{N} n} .
$$

If a single oscillation of a cosine signal with amplitude $\left|A t_{a b}\right|$ is sampled with $N_{\varphi}$ steps, the amplitude in the first non-zero frequency component of a discrete Fourier transform is $s=\frac{1}{2} N_{\varphi}\left|A t_{a b}\right|$. Gaussian white noise with a standard deviation $\sigma_{a}$ results in a mean amplitude in same component of $n=\sqrt{\frac{\pi}{4}} \sqrt{N_{\varphi}} \sigma_{a}$. However, the noise $n$ has a random phase with respect to the signal $s$, leading to the mentioned deviation from the optimal phase. The probability density function of the phase deviation $\theta=\phi_{\sigma}-\phi_{a}$ is given by [98]

$$
p(\theta ; k)=\frac{e^{-\frac{1}{2} k^{2}}}{2 \pi}+\frac{k \cos (\theta)}{\sqrt{2 \pi}} e^{-\frac{1}{2} k^{2} \sin ^{2} \theta} \Psi(k \cos (\theta))
$$

with $k=s / n$ and

$$
\Psi(x)=\frac{1}{\sqrt{2 \pi}} \int_{-\infty}^{x} d y e^{-\frac{1}{2} y^{2}}
$$



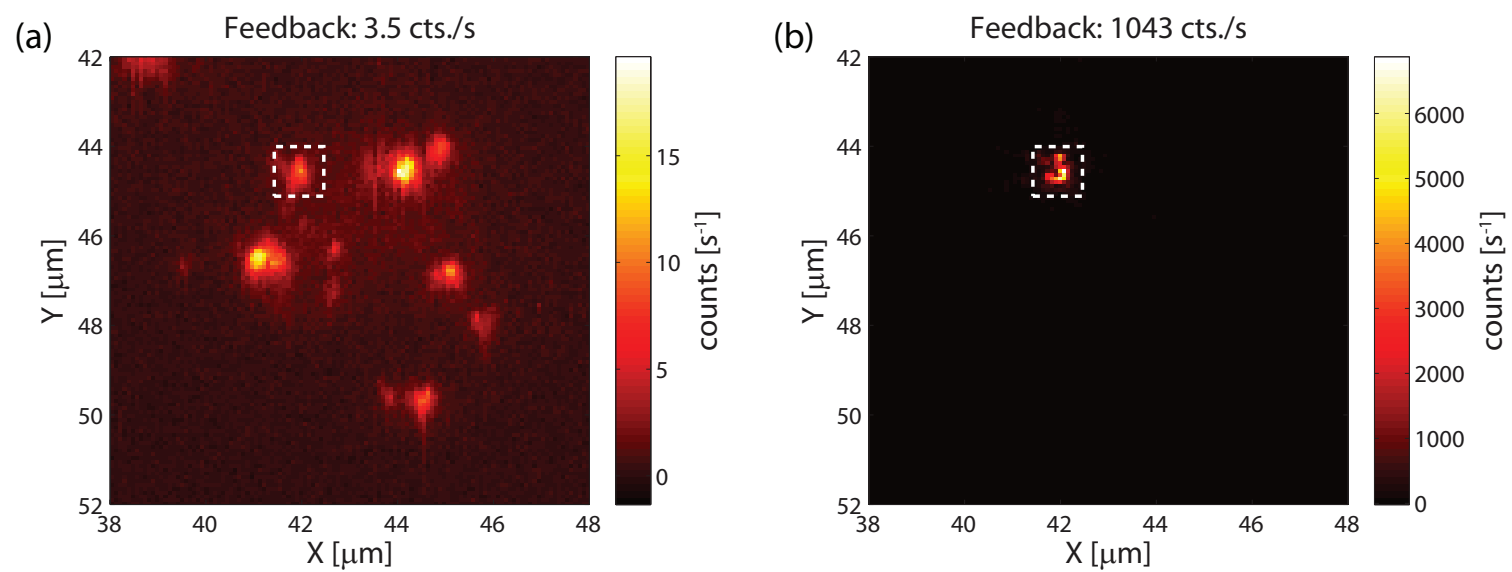

Figure 5.2: Sample backside imaged at the second harmonic wavelength $\lambda=420 \mathrm{~nm}$. (a) Average image obtained from 200 images, each with a different randomly generated illuminating phase pattern. Several nanocrystals are visible in the field of view. (b) Image after wavefront shaping. The feedback signal for the optimization was the average count rate in a square of $1 \mu \mathrm{m} \times 1 \mu \mathrm{m}$ around the position of the selected particle, indicated by the dashed square. Here, the feedback signal was enhanced by a factor of $\eta_{\exp }=3.0 \cdot 10^{2}$.

Consequently the phase correlation can be calculated by

$$
\gamma\left(\left|A t_{a b}\right|, \sigma_{a}, N_{\varphi}\right)=\int_{0}^{2 \pi} d \theta \cos (\theta) p\left(\theta ; k\left(\left|A t_{a b}\right|, \sigma_{a}, N_{\varphi}\right)\right) .
$$

When both contributions are present, the ensemble averaged squared amplitude of the first non-zero frequency Fourier component is written as

$$
\left\langle\left|\mathrm{FT}_{1, a}\right|^{2}\right\rangle=\frac{1}{4} N_{\varphi}^{2}\left\langle\left|A_{a} t_{a b}\right|^{2}\right\rangle+N_{\varphi}\left\langle\sigma_{a}^{2}\right\rangle .
$$

Similarly, the second component squared, $\left\langle\left|\mathrm{FT}_{2, a}\right|^{2}\right\rangle$, will be the sum of the contribution of the noise, $N_{\varphi}\left\langle\sigma_{a}^{2}\right\rangle$, and, according to Eq. (5.5), a contribution proportional to $\left\langle\left|A_{a} t_{a b}\right|\right\rangle^{4}$. The latter contribution, since it is amplified less by the other segments (see Eq. (5.5)), is expected to be about a factor $N_{s}^{2}$ lower compared to the first term on the right hand side of Eq. (5.18) and should therefore be negligible. Experimentally determined values $\left\langle\left|\mathrm{FT}_{2, a}\right|^{2}\right\rangle$ can therefore be used to calculate the noise level, with which $\left\langle\left|A_{a} t_{a b}\right|^{2}\right\rangle$ can be determined from Eq. (5.18) using the experimental values $\left\langle\left|\mathrm{FT}_{1, a}\right|^{2}\right\rangle$.

\subsection{Results and discussion}

\subsubsection{Spatiotemporal focus on a single nanocrystal}

Figure 5.2(a) depicts the experimentally observed average image of several nanocrystals at the sample backside, which is the average of 200 images, each with a different randomly generated illuminating phase pattern. A number of isolated sources of second harmonic radiation are visible in the field of view. The spot sizes are larger than 

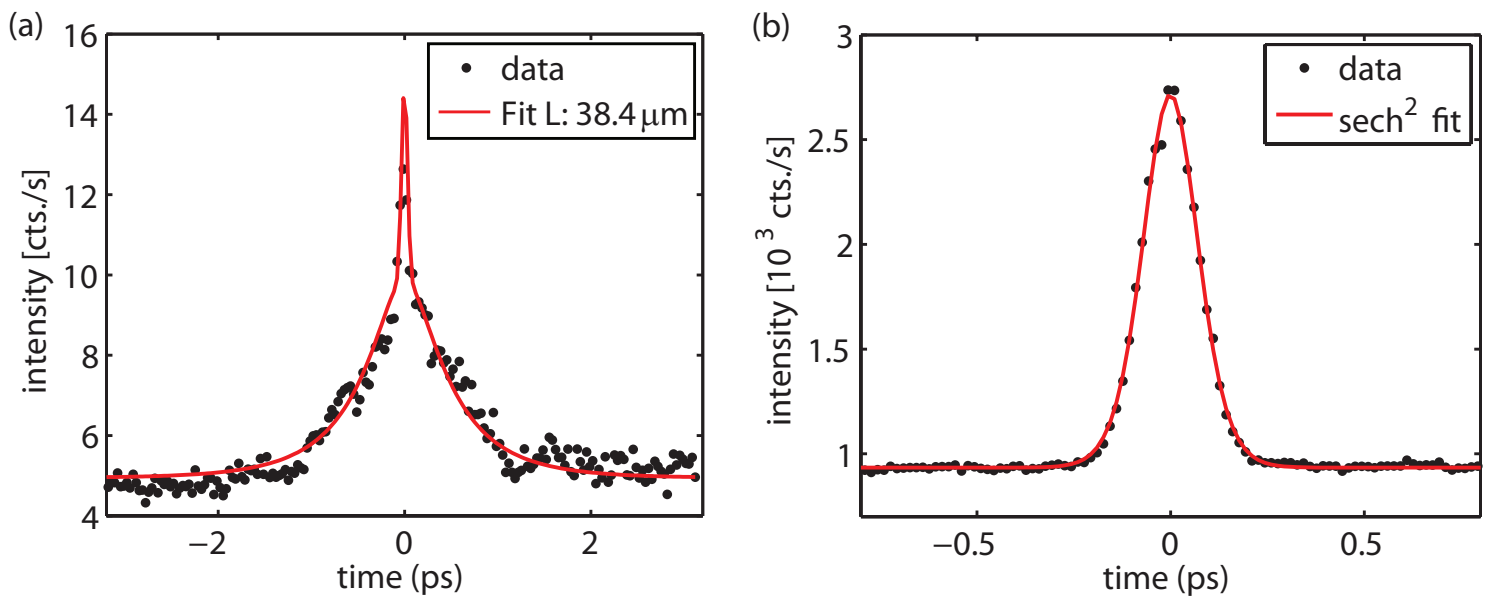

Figure 5.3: Second harmonic autocorrelation measured on an individual nanocrystal at the backside of the sample. The graphs show the mean count rate in a $1 \mu \mathrm{m} \times 1 \mu \mathrm{m}$ square around particle position. In particular, the measurements for particle 5 are shown (see table 5.1). (a) Autocorrelation before wavefront shaping. The data shows a 3:2:1 contrast ratio between the quickly decaying correlation of the coherent part of the speckle pulse followed by a slower decay of the incoherent part of the speckle pulse towards the background. The data is fitted according to that model of Eq. (5.2). The only free parameter of the fit is the thickness of the medium. (b) Autocorrelation measured on nanocrystal at the backside of a slab of disordered silica after wavefront shaping. The FWHM of the fit based on the autocorrelation of a sech ${ }^{2}$ shaped pulse is $168 \mathrm{fs}$, indicating a pulse duration of the fundamental pulse of $109 \mathrm{fs}$ at the particle position.

the nanocrystals themselves, which we explain by scattering from neighboring silica particles.

We performed the WFS experiment for five of the particles in the field of view and a sixth particle at a different position on the sample (not shown). As feedback signal we use the average count rate from a square of $1 \mu \mathrm{m} \times 1 \mu \mathrm{m}$ around the position of the selected particle. The size of the feedback area was chosen to balance between the collection of the largest possible amount of the scattered SHG signal and the increasing influence of camera noise with an increasing feedback area. The feedback signal increases largely during the first three WFS sequences and typically converges to its maximum value after the second sequence with the highest chosen segmentation $(\mathrm{N}=768)$.

For all particles the integrated feedback signal was significantly increased (by a factor $\eta_{\exp }=0.7 \cdot 10^{2}$ to $5.5 \cdot 10^{2}$, see table 5.1). The image after optimization for one of the particles is shown in Fig. 5.2(b). We observed that for different nanocrystals the focus of the detection objective had to be moved towards the scattering medium (estimated adjustment in the range of up to a few $\mu \mathrm{m}$ by the manual adjuster) to obtain the smallest spot size on the camera after optimization. For different particles we observed spot sizes (FWHM of a two-dimensional Gaussian fit) from $0.2 \mu \mathrm{m}$ (close to the diffraction limit) to about $0.6 \mu \mathrm{m}$, where bigger spots were typically more of an irregular shape rather than a homogeneous spot. These observations reflect the distribution of the nanocrystals in the silica layer and the resulting scattering of the SHG radiation by surrounding silica particles. 


\begin{tabular}{|c|c|c|c|c|}
\hline Part. \# & $\tau_{\text {pulse }}(\mathrm{fs})$ & $\eta_{\exp }$ & $\eta_{\text {model }}$ & $\eta_{\mathrm{cw}}$ \\
\hline & \multicolumn{2}{|c|}{ experiment } & & \\
\hline \hline 1 & 110 & $2.5 \cdot 10^{2}$ & $3.1 \cdot 10^{2}$ & $1.6 \cdot 10^{4}$ \\
\hline 2 & 102 & $0.7 \cdot 10^{2}$ & $3.2 \cdot 10^{2}$ & $1.7 \cdot 10^{4}$ \\
\hline 3 & 111 & $2.7 \cdot 10^{2}$ & $2.7 \cdot 10^{2}$ & $1.4 \cdot 10^{4}$ \\
\hline 4 & 104 & $0.7 \cdot 10^{2}$ & $2.9 \cdot 10^{2}$ & $1.5 \cdot 10^{4}$ \\
\hline 5 & 109 & $3.0 \cdot 10^{2}$ & $3.8 \cdot 10^{2}$ & $2.0 \cdot 10^{4}$ \\
\hline 6 & 109 & $5.5 \cdot 10^{2}$ & $6.5 \cdot 10^{2}$ & $2.4 \cdot 10^{4}$ \\
\hline
\end{tabular}

Table 5.1: Summary of the results of six wavefront shaping experiments, each with a different particle visible in Fig. 5.2. The pulse duration $\tau_{\text {pulse }}$ is calculated from the Gaussian fit to the autocorrelation curve after optimization. The experimentally observed enhancement $\eta_{\text {exp }}$ is calculated by the ratio of the average count rate in a $1 \mu \mathrm{m} \times 1 \mu \mathrm{m}$ square around the particle position after and before optimization. For each particle we calculated the enhancement $\eta_{\text {model }}$ of the time-integrated $\mathrm{SH}$ according to our model, calculated by $\eta_{\text {model }}=c_{\tau} c_{\alpha} c_{r} \eta_{\mathrm{cw}}$. The theoretical enhancement of the $\mathrm{SH}$ in a hypothetical continuous wave experiment $\eta_{\mathrm{cw}}$, is calculated based on the experimentally determined amplitude contribution. The factor $c_{\tau}$, which corrects the enhancement for speckle pulses, was calculated using (Eq.) 5.10, where the sample thickness to model $\langle I(t)\rangle$ is determined from the fits of the autocorrelation in Fig. 5.3(a). The particles 1-5 were all located in close vicinity, where we assume a constant thickness and an average value $c_{\tau}=0.12$ was determined. Particle 6 was located on a different spot on the sample, where we obtained $c_{\tau}=0.17$. The factors $c_{\alpha}=0.28$ and $c_{R}=0.57$ include the polarization and the susceptibility tensor and the focal volume, respectively (see sections 5.3.2 and 5.3.2).

The autocorrelation measurements from the particle in Fig. 5.2(b) before and after WFS are shown in Fig. 5.3. The behaviour of the autocorrelation before WFS is fitted with the speckle autocorrelation model explained above (see Eq. (5.2)). The autocorrelation fit function is calculated based on average time-resolved transmission $\left\langle I_{\mathrm{d}}(t)\right\rangle$ at the particle position according to [99] using the experimental parameters (mean free path $l=3.5 \mu \mathrm{m}$, extrapolation length ratio at silica-air interface $z_{e 1}=$ 1.38 and $z_{e 2}=0.71$ at the silica-glass interface, effective refractive index $n_{\text {eff }}=1.25$, beam waist of illumination $w_{I}=150 \mathrm{~nm}$ and detector size $w_{D}=100 \mathrm{~nm}$ ). Since the thickness of the sample varies between about $25-50 \mu \mathrm{m}$, we use the thickness $L$ as fit parameter. After WFS, the autocorrelation shows a sharp peak, demonstrating that the light is focused as a short pulse. A contribution of the non-optimized part of the pulse (see Sec. 5.3.2), which could be expected as a small signature next to the correlation peak of the focused part, is not visible due to a present higher noise level. The measured autocorrelation can be fitted very well with the autocorrelation based on a sech ${ }^{2}$ pulse shape. We calculate the pulse duration from the fit with the well-known deconvolution factor $\tau_{\text {pulse }}=0.65 \tau_{A C}, \tau_{A C}$ being the FWHM of the fit. We have summarized the results obtained from all six particles in Table 5.1. For all pulses, the pulse shape changed from speckle pulse before the optimization to a single short pulse after WFS with a duration $\tau_{\text {pulse }}$ ranging between $102 \mathrm{fs}$ to $111 \mathrm{fs}$. 
(a)

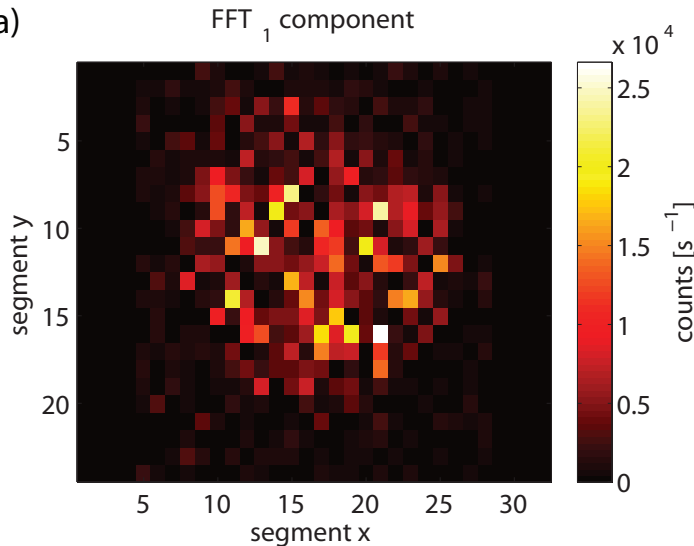

(b)

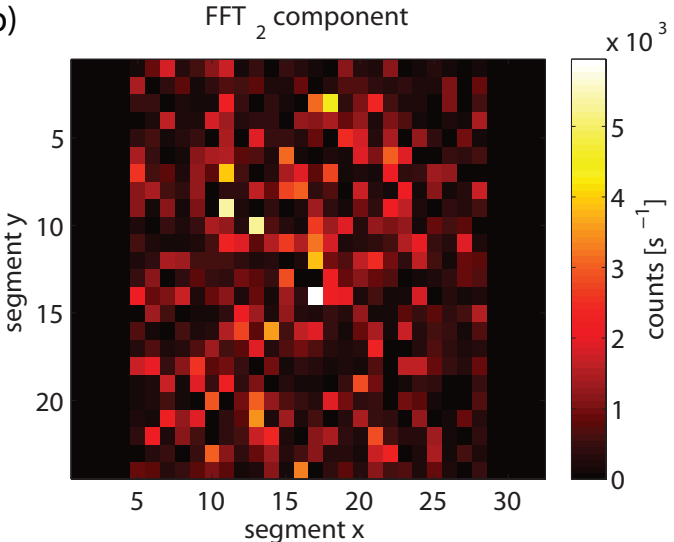

Figure 5.4: Fourier analysis of the feedback signal as a function of phase $\varphi_{a}$ per segment during a sequence of the wavefront shaping experiment. The graph on the left (a) shows the squared amplitude of the first non-zero frequency Fourier component $\left|\mathrm{FT}_{1}\right|^{2}$ calculated by Eq. (5.14), the graph on the right (b) depicts the second component $\left|\mathrm{FT}_{2}\right|^{2}$. The distributions are used to determine the amplitude distribution of the contributing segments and the noise level (see sections 5.3.2 and 5.4.2).

\subsubsection{Comparison of the measured and the modeled enhance- ment}

In the following we compare the measured enhancement with the model introduced above. In order to evaluate Eq. (5.13) and Eq. (5.10), we determine the amplitude distribution $\left|A_{a} t_{a b}\right|$ and the average time-resolved transmission $\left\langle I_{\mathrm{d}}(t)\right\rangle$ from the experimental data.

In order to calculate $\eta_{\mathrm{cw}}$ from Eq. (5.13), we determine the amplitude distribution $\left|A_{a} t_{a b}\right|$, the noise $\sigma_{a}$ and the phase correlation $\gamma\left(\left|A t_{a b}\right|, \sigma_{a}, N_{\varphi}\right)$ from the data following the considerations of Sec. 5.3.2. In particular, we analyze the recorded feedback values during the WFS experiment, during a sequence after the algorithm already has converged. We calculate the Fourier transform of the phase scan for each segment by Eq. (5.14). The calculated components $\left|\mathrm{FT}_{1, \mathrm{a}}\right|^{2}$ and $\left|\mathrm{FT}_{2, \mathrm{a}}\right|^{2}$ on the SLM for particle 5 are shown in Fig. 5.4. The distribution of the components $\left|\mathrm{FT}_{1, \mathrm{a}}\right|^{2}$ reflects the Gaussian spatial profile of the illuminating beam on the SLM and the random transmission through the sample. The components $\left|\mathrm{FT}_{2, a}\right|^{2}$ show a weak dependence on the segment position, with slightly higher average values for the center segments of the SLM. Furthermore, we find the phase values of $\mathrm{FT}_{1, a}$ and $\mathrm{FT}_{2, a}$ to be uncorrelated. Using Eq. (5.18), we obtain $\left\langle\left|A_{a} t_{a b}\right|^{2}\right\rangle$ by fitting the values of $\left|\mathrm{FT}_{1, a}\right|^{2}$ in Fig. 5.4(a) with a two-dimensional Gaussian after subtracting the noise $\left\langle\sigma_{a}^{2}\right\rangle$, which itself was determined from a two-dimensional Gaussian fit of $\left|\mathrm{FT}_{2, a}\right|^{2}$. In order to calculate $\gamma\left(\left|A t_{a b}\right|, \sigma_{a}, N_{\varphi}\right)$, we numerically evaluate Eq. (5.17) for each segment using the noise $\sigma_{a}$ and the amplitude $\left\langle\left|A_{a} t_{a b}\right|\right\rangle$ obtained from the fits. We successfully tested our analysis on a simulated WFS experiment (see appendix 5.A). In the simulation the enhancement is correctly calculated with an accuracy of about $20 \%$ for all noise levels as long as the convergence of the optimization is not spoiled by the noise.

It is noteworthy that the components $\left|\mathrm{FT}_{2, a}\right|^{2}$ in Fig. 5.4(b) show a weak dependence on the segment position, which can be caused by several effects. Firstly, 
cross-talk between pixels of the SLM and diffraction effects for larger phase shifts between neighboring segments can cause a noise term which depends on the intensity present on the segment. Secondly, non-linear effects which are not considered in the above model might be present, such as two-photon absorption in the disordered medium. For all cases, we assume that the linear transmission will be affected by noise or a noise-like contribution of the same magnitude and therefore treat the contributions $\left|\mathrm{FT}_{2, a}\right|^{2}$ as noise term to analyze Eq. (5.18).

In order to evaluate the integral in Eq. (5.10) the speckle correlation time $\tau_{\mathrm{c}}$ is calculated as the half width at half maximum of the measured Fourier transformed laser spectrum. We model the average time-resolved transmission $\langle I(t)\rangle$ at the particle position according to [99] as described above, with the thickness L obtained from the fit to the autocorrelation measurements (Fig. 5.3(a)) .

With the calculated amplitude enhancement $\eta_{\mathrm{cw}}$, and the factors $c_{\tau}, c_{\alpha}$ and $c_{R}$, we calculate the enhancement predicted by our model, $\eta_{\text {model }}=c_{\tau} c_{\alpha} c_{r} \eta_{\mathrm{cw}}$. For all particles, the calculated values are listed in table 5.1. For the particles 1, 3, 5 and 6 the modeled enhancement predicts the experiment value within the accuracy of our model. For particles 2 and 4 it overestimates the enhancement by a factor 4.6 and 4.1 respectively. An overestimation of this magnitude occurs, if two or more particles are placed in direct vicinity, a case which is difficult to identify from the camera images. All particles would contribute to the average reference signal, which would decrease the calculated enhancement accordingly. Furthermore, an overestimation of the enhancement is not surprising considering the composition of the signal which is being optimized. For a given experimental realization of the sample and the nanocrystal in our experimental setup, the feedback signal is solely a function of the phase values set on the SLM. However, next to a global maximum, for which we perform our calculations, the feedback function has a vast number of local maxima into which the algorithm will converge for a given set of starting phase values. A specific example is the factor $c_{\tau}$ which is calculated for the ideal case, that the optimized pulse is formed exactly at the moment in time where the average time-resolved transmission has its maximum. However, in the experiment the point in time at which the optimized pulse is formed will most likely not coincide with the maximum, leading to a lower observed enhancement. Applying different algorithms for the wave front shaping optimization, e.g., a genetic algorithm $[147,148]$, could be a way to increase the experimentally achieved enhancement. We assume that a similar experiment with lower complexity, e.g., by use of nanoparticles with a less complex second-order susceptibility (see Sec. 5.3.2) or by using quasi-monochromatic light, will produce enhancement values which are predicted even more accurately by our model.

\subsubsection{Pulse duration after WFS}

Here we discuss the observed duration of the focused pulses after WFS. The pulse duration, derived from the autocorrelation after optimization varies only slightly for different particles, between $102 \mathrm{fs}$ to $111 \mathrm{fs}$. Altogether the values are longer than the correlation time $\tau_{\mathrm{c}}=52 \mathrm{fs}$ calculated from the measured spectrum of the laser. This observation resembles that made by the first study of (far-field) spatiotemporal focusing through a turbid medium [135]. As shown in [135], the optimization 
Spatiotemporal focusing in turbid media by wavefront shaping with nonlinear feedback

process leads to spectral narrowing. The spatial optimization with the SLM, which for each segment induces a phase-shift independent of frequency, cannot optimize all frequencies equally well. The optimization is biased towards those frequencies which contribute higher to the feedback signal which are on average the frequencies in the center of the spectrum. In the approach of [135], linear time-resolved optical gating is applied to generated the feedback signal, and the resulting spectrum of the focused pulse can be quantitatively predicted exactly from the known laser spectrum.

Following this consideration, we can give an upper limit for the final pulse duration for the present experiment. Here the feedback signal is based on second-harmonic generation, of which the intensity is proportional to the field amplitude at the fundamental frequency to the power of four. Using this dependence as the weighting factor for the transmission of the original spectral amplitude results in a calculated pulse duration of $122 \mathrm{fs}$, assuming that the focused pulses are bandwidth limited. However, this calculation simplifies the fact that spectral amplitude of the second harmonic radiation depends both on the spectral amplitude and spectral phase function of the fundamental radiation. The exact duration of the part of the speckle pulse which is optimized and respectively its spectral composition at the beginning and their dynamics during the optimization procedure depends in a complex way on the starting conditions and the exact transmission coefficients. From the experimental observations we conclude that the spectral function will in general not converge to the calculated limit but remain slightly broader, resulting in shorter pulse duration than the calculated value. Any pulse duration in between the original bandwidth limit and the upper limit can be reached, with the most likely final pulse durations in the observed range.

\subsubsection{Peak-to-background ratio}

For future applications of the presented experiment, it is useful to estimate the temporal peak-to-background ratio of the focused pulses. The focused pulses are concentrated in a narrow time window as discussed above, but will still be preceded or followed by a small diffuse speckle contribution. For the generated second-harmonic pulse, the theoretical value for the ratio is given by the calculated enhancement at a single point in time (Eq. (5.8)) . From the experimental enhancement values divided by the effective number of independent speckle grains $c_{\tau}$, we calculate a peak-tobackground ratio between $6 \cdot 10^{2}$ and $3.2 \cdot 10^{3}$ for the investigated particles. The analog peak-to-background ratios for the fundamental intensity are approximately given by the square root of these values.

\subsection{Conclusions}

We have demonstrated spatiotemporal focusing inside scattering media using SHG in nanocrystals as a feedback signal for wavefront shaping. We developed a model which predicts the observed increase of the feedback signal and the pulse duration at the focus well. Our method provides a means to locally generate a short coherent light pulse with a high signal to background ratio inside a complex scattering medium. This provides an exciting tool for various applications of the control of light propagation 
in complex and especially nonlinear media, and to study light propagation in the presence of strong multiple scattering. 


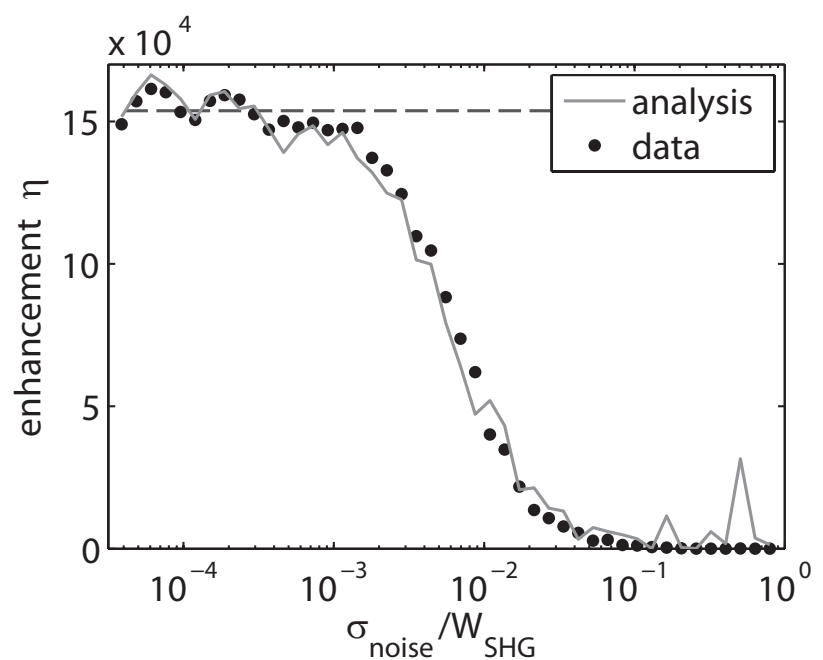

Figure 5.5: Enhancement of the second harmonic feedback signal $\eta$ as a function of the relative noise on the detected SHG signal from a simulated WFS experiment as described in appendix 5.A (black dots). The grey line is the result from the analysis described in Sec. 5.4.2. The dashed line is the average enhancement without noise.

\section{A Test of noise analysis by simulations}

In Sec. 5.4.2 we described an analysis method to estimate how much the experimentally achieved enhancement is affected by noise. In this appendix, we present a successful test of this analysis method with data from a simulated wavefront shaping experiment.

The main purpose of the test is to confirm that the analysis is valid for a wide range of noise values and also for a non-equal amplitude distribution $A_{a}$ of the SLM segments. Regarding other aspects, the simulation was simplified compared to the situation in the experiment in order to shorten the computation time: The simulation was performed assuming a time-independent (i.e., monochromatic), scalar electric field. Furthermore, only a single point in transmission was assumed to generate a second-harmonic response.

The procedure for the simulation was as follows. The simulation was initiated by two steps:

1. The amplitude contribution $A_{a}$ for each segment was calculated to reflect the spatial profile of the Gaussian beam on the SLM. The WFS simulation was performed with $\mathrm{N}=768$ segments, equivalent to the experiment.

2. A set of random amplitude transmission coefficients from a circular complex Gaussian distribution was generated which connect the field amplitude at the SLM and the point of SHG generation.

A single measurement of the SHG signal was simulated by the following steps:

3. The generated SHG power was calculated according to Eq. 5.3 and Eq. 5.4, using $E_{a}=A_{a} e^{i \phi_{a}}$, where $\phi_{a}$ are the phase shifts applied by the SLM. All constants of the equations were set as one, as only relative values are of interest. 
4. A random noise term was added to the detected SHG power. The noise term is generated from a Gaussian distribution with a standard deviation proportional to the SHG signal.

We simulated a series of wavefront shaping experiments for different noise levels. Each experiment was performed with three full iterations with the standard sequential algorithm, where the third iteration was used for the analysis.

The results are shown in Fig. 5.5. For low noise levels, the enhancement is constant and close to the theoretical value in absence of noise (dashed line). With increasing noise levels, the enhancement is reduced. We note that the optimization did not converge to a stable value for relative noise levels above a certain threshold (here $\approx 0.08$ ). Below that threshold, the enhancement predicted by analyzing the phase scan data agrees within $20 \%$ with the data. Despite the simplifications made in the simulation, we assume the analysis method is equally valid for the experiment: Firstly, the relative noise level in the experiment is below 0.01 and convergence of the optimization is observed. Secondly, the phase scan data analyzed in Sec. 5.4.2 corresponds to the situation in which the SHG signal originates to the largest part from a single spatiotemporal focus, which is comparable to the single-point focus in the simulation. 



\section{Spatial and temporal focus on nanocrystals hidden deep inside a random medium}

We experimentally demonstrate focusing of ultra-short laser pulses on a nanoparticle deep inside a disordered medium. We fabricate a slab structure from randomly assembled silica beads, in which we embed nanocrystals which are efficient for second-harmonic generation (SHG). Light transport of the fundamental radiation is in the transition regime between ballistic transport and diffusion. In optimizing the wavefront of the incident fundamental radiation, the SHG signal scattered from the nanocrystals is increased by more than four orders of magnitude. By adapting the degrees of freedom of the incident wavefront, the ratio between diffuse and ballistic light contributing to the focus can be controlled; this finding makes the studied system highly interesting for testing of models for light scattering. We envision that the presented approach will provide a building block for a wide range of novel experiments in disordered and ordered photonic systems and metamaterials.

\subsection{Introduction}

Scattering of light from inhomogeneities in a medium often impedes fundamental studies and desirable applications in photonics and metamaterial research and biomedical imaging. In a thick inhomogeneous medium the ballistic component of the light is converted into multiply scattered diffuse light. Spatially, light in a welldefined mode of the input wavefront is randomly scattered into the large number of output modes, giving rise to the well-known speckle interference patterns. This speckle pattern changes quickly as a function of frequency of the input light, due to the broad path length distribution inside the medium [90]. Temporally, incident ultrashort pulses are strongly distorted and widely stretched while propagating through 
the medium [117]. By conventional means it is not possible to concentrate light either spatially or temporally in such a medium.

However, a wide range of techniques rely on spatiotemporal focusing of light and are consequently restricted to the ballistic regime. Being able to remove or compensate the spatiotemporal distortions would open the door for novel experiments and applications involving ultra-fast lifetime measurements, nonlinear spectroscopy [114] and multi-photon imaging [3] inside random media. Finding new concepts to control light propagation in complex multiple scattering systems is further of interest for application in designed photonic systems [149] or metamaterials [150].

The invention of wavefront shaping $[13,17]$ demonstrated that monochromatic light can actually be focused even when the medium is many times thicker than the mean free path. The approach is based on iterative shaping of the input wavefront to optimizing a feedback signal connected to the intensity in the desired output mode. If light propagation in the medium is linear, the transmission coefficients connecting the input and output modes can also be measured directly or by means of phase conjugation for focusing or image transmission [18][20][26, 27]. Wavefront shaping quickly found numerous applications such as in high-resolution imaging [24], optical trapping[121] and optical coherence tomography [34].

The iterative wavefront shaping scheme offers the advantage that it is not necessary to access the wave field amplitude directly in the output mode; a signal increasing with increasing intensity in the desired output mode is sufficient to obtain focusing. Using fluorescent probe particle enables to focus monochromatic light deep inside the scattering medium [14]. A promising variation of this concept for biomedical applications is to create the local intensity probe by tagging the light traveling through intended focal region by ultrasound [36]. Closely related studies have earlier been conducted with acoustic waves and microwaves; for those types of waves, the wave field can readily be measured and generated by ultrasound transducers and antennas respectively for time reversal experiments [39][42].

In a recent experiment wavefront shaping was extended to broadband light for spatial and temporal focusing of ultra-short pulses through scattering media [135]; the feedback signal is provided by time-resolved detection of the transmitted speckle field at the intended focal spot. Optimizing the incident wavefront for longer or for shorter light paths through the medium allows controlling the arrival time of the focused pulse. Remarkably, comparable focusing in transmission medium can even be achieved without explicit time-resolution of the feedback signal when two-photon fluorescence is employed [109]. A second-harmonic response from nanocrystals has been successfully applied for spatiotemporal focusing in small volumes directly at the medium interface [151]. However, in all present the focal point lies outside the medium or at its interface and can be directly imaged.

In this chapter, we experimentally demonstrate focusing of ultra-short laser pulses deep inside a disordered medium by wavefront shaping employing second-harmonic generation from embedded nanocrystals. In particular, the approach is applied to a system in which the light is partially transported ballistically and partially undergoes multiple scattering. In the following section, we first describe the principle of spatiotemporal focusing by wavefront shaping with nonlinear feedback and then provide a brief description of the theoretical model for the observed increase of the feedback 
(a)

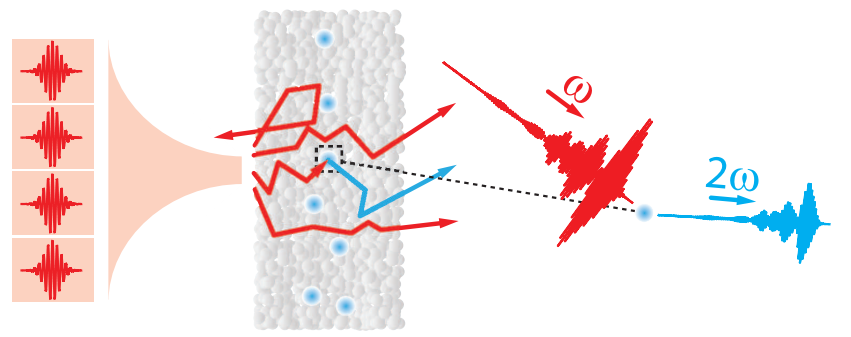

(b)

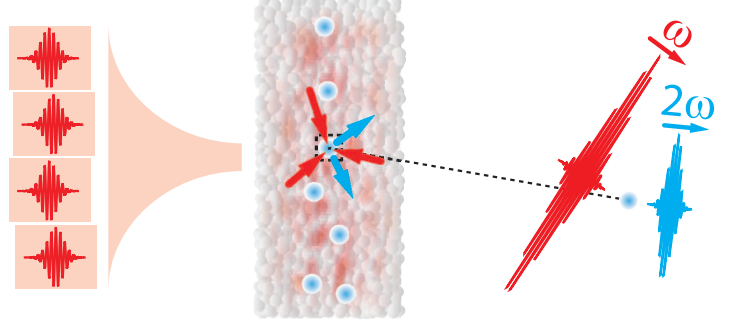

Figure 6.1: Spatiotemporal focusing inside the medium by wavefront shaping. (a) Illumination by arbitrary wavefront. At the position of the second-harmonic active nanocrystal, a feeble speckle pulse is converted to the double frequency. (b) Illumination by optimized wavefront. At the position of the nanocrystal, the light is both spatially and temporally focused, and a strong second harmonic signal is generated.

signal. The framework of the model was developed and extensively presented in the context of a wavefront shaping experiment in transmission configuration in chapter 5. In Sec. 6.3, we describe the experimental methods with an emphasis on the characterization of the light scattering properties of the fabricated sample, followed by the results in Sec. 6.4. In Sec. 6.5 we discuss the results. We have strong evidence that a single spatiotemporal focus is created inside the medium. Furthermore, our method allows controlling the ratio of ballistic and diffusive contributions to the spatiotemporal focus.

\subsection{Theory}

The underlying mechanism of the experiment is described as follows. The incident wavefront is segmented into $N$ segments whose phase can be adjusted individually with a phase offset between zero and $2 \pi$ at the center wavelength of the laser. Light reflected from a single segment (index $a$ ) on the SLM is transmitted to the probe nanocrystal at position $b$. In a simple scalar picture, it gives rise to the complex field $E_{b}(t)=h_{a b}(t) \otimes E_{a}(t)$, where the right-hand side of the expression denotes the convolution of the complex impulse response $h_{a b}(t)$ with the incident pulse $E_{a}(t)$. The phase of $E_{a}(t)$ can be modified by a time-independent phase shift $\Delta \Phi_{a}$ via the SLM. The total electric field set up at the particle position is given by

$$
E(t)=\sum_{a=1}^{N} h_{a b}(t) \otimes E_{a}(t) e^{i \Phi_{a}}
$$

For an arbitrary set of phases $\Delta \Phi_{a}$, the field $E(t)$ manifests as a random speckle pulse (Fig. 6.1a). In the nanocrystal the field induces a second-order polarization 
$P_{2 \omega}(t)=\chi_{2} E^{2}(t)$, and the particle radiates a second harmonic power $W_{2 \omega}(t) \propto$ $\left|P_{2 \omega}(t)\right|^{2}$, assuming that the quasi-static limit holds.

The aim is to adjust the wavefront, i.e., the set of phase shifts $\Phi_{a}$, such that a single moment in time $t_{0}$ the field $E\left(t_{0}\right)$ is maximized (Fig. 6.1b). This results in the forming of an enhanced short pulse at time $t_{0}$ on top of a feeble speckle background at all other times[135]. To achieve this we exploit the fact that the phase of the speckle field of a single segment, $\arg \left\{E_{b}(t)\right\}$, at two points in time is uncorrelated, if they are temporally separated further than the coherence time given by the pulse spectrum. As a consequence, the correct adjustment of the phase of segments at time $t_{0}$ will be accompanied by a remaining random phase distribution of a typical speckle pulse at other times.

Although we cannot access the field $E(t)$ directly for our purpose, the correct wavefront can be indirectly found by maximizing the total second-harmonic generation (SHG) from the nanocrystals (see chapter 5 or [151]), which can be detected as diffuse light from outside the medium. Due to the nonlinear dependence of the process on the incident light field, the optimization is not only biased to spatially focus on the 'guide star' crystal, but is also strongly biased towards focusing in the time domain. From a theoretical point of view and in view of earlier transmission experiments we know the optimization will converge to a single spatiotemporal focus. A situation, in which two independent points with an uncorrelated set of transmission coefficients would be simultaneously optimized, is unstable and would converge to a single focus.

Based on the picture of a single spatiotemporal focus, we can calculate the expected enhancement of the SHG intensity. For the experimental realization we assume that the radiation detected outside the medium is a constant fraction of the SHG from the embedded nanocrystals. The average enhancement of this feedback signal $S_{2 \omega}$ is given by [151]

$$
\begin{aligned}
\eta & =\frac{\left\langle S_{2 \omega}\right\rangle_{\mathrm{WFS}}}{\left\langle S_{2 \omega}\right\rangle} \\
& \approx 1+\gamma\left(0.5(\alpha \beta)^{2} N^{2}+3(\alpha \beta)[1-(\alpha \beta)] N\right)
\end{aligned}
$$

with

$$
\alpha=\frac{\left\langle\left|A_{a}\right|\right\rangle^{2}}{\left\langle\left|A_{a}\right|^{2}\right\rangle}, \beta=\frac{\left\langle\left|h_{a b}\left(t_{0}\right)\right|\right\rangle^{2}}{\left\langle\left|h_{a b}\left(t_{0}\right)\right|^{2}\right\rangle}
$$

and the control factor $\gamma$. In Eq. 6.3 we neglect terms depending on $N$ with negative exponents. Since the surface of the SLM is illuminated by a Gaussian beam, the segments do not contribute equally to the focus. This inhomogeneity is accounted for by averaging over the amplitude distribution $A_{a}=\max \left(\left|E_{a}(t)\right|\right)$, given by the Gaussian amplitude distribution of the illuminating beam incident on the spatial light modulator. For the response function $h_{a b}\left(t_{0}\right)$, we assume a circular Gaussian distribution to calculate the ratio of the ensemble average, resulting in $\beta=\pi / 4$.

The control factor $\gamma$ in Eq. 6.3 accounts for the fact that in the experiment only a certain fraction of the feedback signal can be controlled and contributes efficiently to the focus. Equation 6.3 with $\gamma=1$ would describe the enhancement of the secondharmonic power generated at one point in space and time for a scalar electric field. In general, second harmonic radiation generated at points in space other than the final 
focus in the sample, and at earlier or later points in time are not optimized; both before and after the optimization, we assume a contribution given by the ensemble average. The control factor $\gamma$ therefore accounts for several effects, which we discussed and identified in our earlier study for the case of a transmission experiment (chapter $5)$.

In detail, the number of independent temporal degrees of freedom $N_{\tau}=\left(\gamma_{\tau}\right)^{-1}$ depends on the average temporal spread of the diffuse light, which is launched at the sample interface and converted by SHG in the nanocrystals. The number of independent spatial degrees of freedom $N_{s}=\left(\gamma_{n} \gamma_{r} \gamma_{\alpha}\right)^{-1}$ has several aspects. Firstly, several nanocrystals may be present in the probed volume inside the slab, accounted by $\gamma_{n}$. Secondly, at the position of the nanocrystal on which the focus is build, the final focal volume can be smaller than the nanocrystal volume, such that only a part of the volume contributes to the focus $\left(\gamma_{r}\right)$. Furthermore, the polarization state of the focus depends on the size and shape of the nanocrystal and its susceptibility tensor; effectively, the different crystal axes will not contribute equally to the focus $\left(\gamma_{\alpha}\right)$. The latter two factors can be calculated numerically (see section 5.3). Adapted to the situation of the present experiment, we write $\gamma$ as the product

$$
\gamma=\gamma_{\tau} \gamma_{n} \gamma_{r} \gamma_{\alpha}
$$

\subsection{Experiment}

\subsubsection{Optical setup}

The optical setup of the experiment is schematically shown in Fig. 6.2. The beam from a mode-locked Ti:Sapphire laser (Ti:Sa; $80 \mathrm{MHz}$ repetition rate, $841 \mathrm{~nm}$ center wavelength, $66 \mathrm{fs}$ pulse duration) is coupled into a Michelson-type interferometer (MI). Subsequently the beam is spatially modulated by a reflective two-dimensional spatial light modulator (SLM; $16 \times 12 \mathrm{~mm}, 800 \times 600$ pixel, phase only $0 \ldots 2 \pi$ ). The modulated beam is projected onto the back-focal plane of an air-immersion objective with a numerical aperture (NA) of 0.9. The scattering sample (see following section

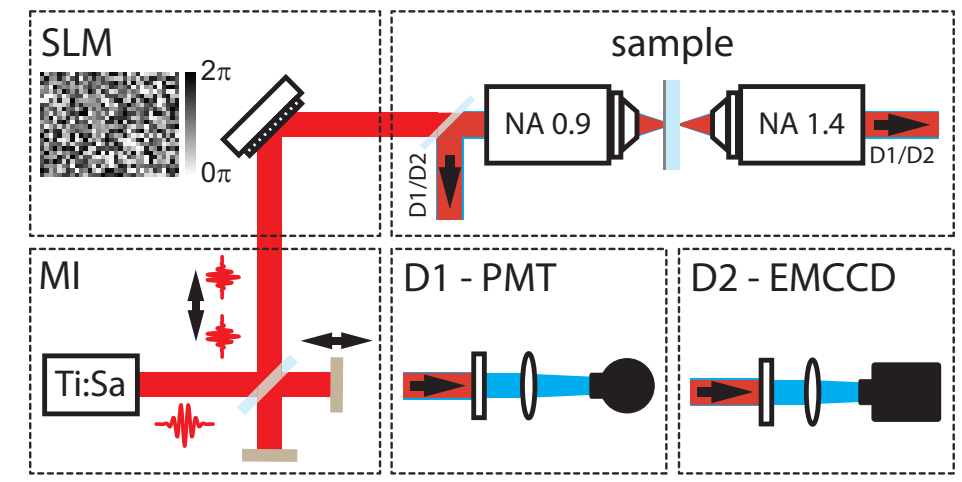

Figure 6.2: Schematic of experiment. (Ti:Sa) Mode-locked titanium-sapphire laser. (MI) Michelson-type interferometer. (SLM) Spatial light modulator. (NA) Objectives with specified numerical aperture. (D1-PMT) detector one; photomultiplier tube. (D2-EMCCD) detector two; electron-multiplying CCD camera. 

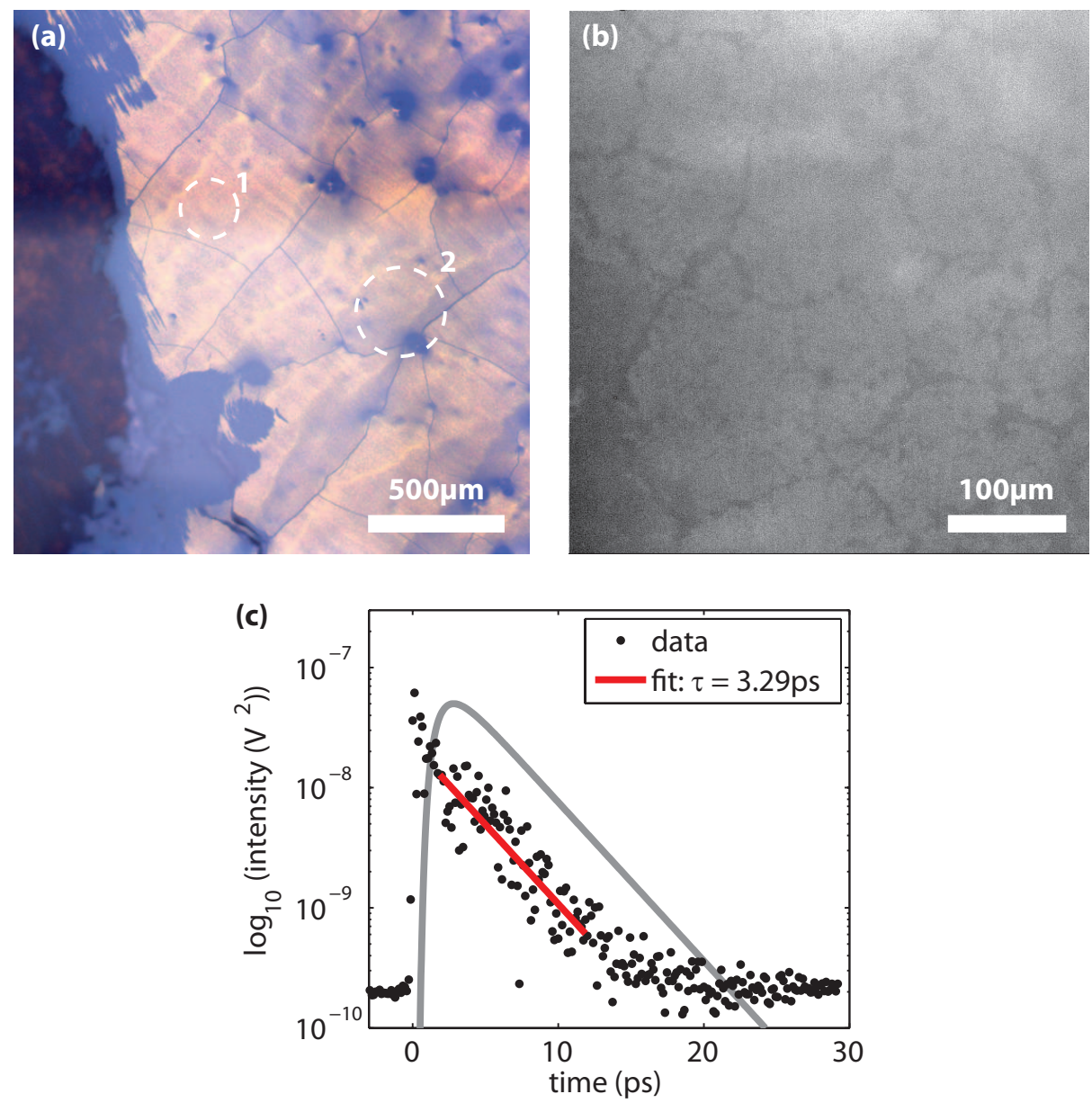

Figure 6.3: Sample characterization. (a) Bright field image of the air-silica interface with epi-illumination. On the right-hand side of the image we see the surface of the disordered silica layer. On the left-hand side we see the glass substrate (out of focus), because part of the silica layer broke off the substrate surface during fabrication. Within the areas of the dashed circles we performed two series of wavefront shaping experiments. (b) Brightfield image of the silica-glass interface with trans-illumination, at a location approximately opposite to the center area in image (a). (c) Time-resolved transmission of the fundamental radiation $(\lambda=840 \mathrm{~nm})$ through the sample, measured with an experimental setup similar to [135]. The red solid line is an exponential fit of the long-time decay. The gray line is the transmission through a slab with the same decay time $(\tau=3.29 \mathrm{ps})$ and the same thickness $(L=82 \mu \mathrm{m})$ assuming diffusive transport calculated by Eq. 2.18. 
for a description) is positioned such that the front interface coincides with the focal plane of the first objective. In the beam path in front of the air-immersion objective there is a pellicle beam splitter for (partial) detection of the reflected light. The incident power on the sample is $25 \mathrm{~mW}$, which corresponds to an average irradiance of $1 \mathrm{MW} / \mathrm{cm}^{2}$ for a tight focus by a flat wavefront. The transmitted light is collected with an oil-immersion objective $(\mathrm{NA}=1.4)$. The back interface of the sample is at the focal distance of this objective. A number of flip mirrors (not shown) allows the detection of either the reflected light or the transmitted light either with a photomultiplier tube (PMT) or an electron-multiplying CCD camera (EMCCD). In all detection pathways, band-path filters can be introduced to selectively detect only the second harmonic radiation at $420 \mathrm{~nm}$ wavelength.

\subsubsection{Sample}

The sample, which is placed between the two objectives, consists of a disordered slab of silica beads (single bead diameter $250 \mathrm{~nm}$ to $500 \mathrm{~nm}$ ) on a standard glass cover slip (thickness $0.17 \mathrm{~mm}$ ). The silica layer was fabricated by successive application of several silica layers from a colloidal dispersion in ethanol. For the first layer, we used beads of $500 \mathrm{~nm}$ diameter and added second-harmonic active nanocrystals in a very low concentration $\left(10^{-5}\right.$ mass fraction). The nanocrystals consist of barium titanate $\left(\mathrm{BaTiO}_{3}\right)$ in a tetragonal crystal structure, which have an average diameter of $200 \mathrm{~nm}$. We used a spray-coating technique to apply the first layer on top of the substrate. Fabrication by the spray-coating technique is a fast, however, the resulting slabs have a relative rough surface (see chapter 5.2). To obtain a smooth top surface, successive layers where made from a 1:1 mixture of $250 \mathrm{~nm}$ and $350 \mathrm{~nm}$ beads, applied by the controlled-drying technique [152-154]. The mixture of differently sized beads was chosen to ensure a disordered deposition and prevent the formation of ordered photonic crystal-like structures during the drying process [155].

We characterize the sample by bright field microscopy, time-resolved transmission measurements and by measuring the ballistic transmission of the fundamental radiation.

The bright field images are depicted in Fig. 6.3. At the air-silica interface in the center of Fig. 6.3a, the layer surface is smooth, forming domains of about $500 \mu \mathrm{m}$ in diameter in size which are separated by visible cracks. By scanning the focus of the microscope, we determine a layer thickness $L=(85 \pm 10) \mu \mathrm{m}$ in the area marked by dashed circle ' 1 '. On the glass-silica interface (Fig. 6.3b), inhomogeneities and cracks are prominent, and intact domains are of the size of $20 \mu \mathrm{m}$ to $100 \mu \mathrm{m}$.

We measured the dynamic transport of light at the fundamental radiation wavelength $(\lambda=840 \mathrm{~nm})$ through the sample by measuring the time-resolved transmission by heterodyne detection (Fig. 6.3c) in an optical setup similar to [135]. For this, we illuminate a small area $(<10 \mu \mathrm{m})$ on the air-silica interface within the area marked with the dashed circle ' 1 ' in Fig. 6.3a, and detect the scattered light in transmission in the forward direction. We averaged the measurements from five different points within the marked area to remove temporal speckle (black dots). We fit the long-term behaviour of the detected flux with an exponential decay (red line), resulting in a decay time $\tau=3.29 \mathrm{ps}$. At early arrival times, more light is detected than would be 
expected by the diffuse transmission through a slab of the same thickness with the same long-term decay constant (gray line), calculated by Eq. 2.18. The long-term decay is well-described by an exponential decay, from which we determine the diffusion constant $D=2.5 \cdot 10^{2} \mathrm{~m}^{2} \mathrm{~s}^{-1}$. From the diffusion constant, we calculate the mean free path $l$ in the medium by $D=v_{E} l / 3$ [10], where $v_{E}$ is the energy velocity in the medium. The energy velocity in turn is obtained by calculating the effective refractive index in the medium $n_{\text {eff }}=1.26$ by Maxwell-Garnett-theory [84], where we use refractive index of the fused silica spheres $n=1.46$ and the filling fraction $f=0.6$ as estimated from SEM images of comparable samples. Using the measured value of $D$ and the computed $v_{E}$, we obtain the mean free path $l_{\exp }=3.2 \mu \mathrm{m}$. The time-resolved transmission at early arrival indicates that the light transport in the sample is not fully described by diffusion. In the following we further investigated on the potential involvement of ballistic transport.

To quantify contributions of ballistic transport we placed the sample in our setup described in Fig. 6.2. We overlapped the focal planes of both objectives and set a flat wavefront on the SLM. In a reference measurement with a bare glass substrate a tight intense focus was visible on the camera. With the sample in place we observed the camera image while translating the sample perpendicular to the optical axis. For most sample positions a faint speckle pattern was visible. At other positions a tight focus was observed on the camera with peak intensities up to $10^{-3}$ relative to the glass reference indicating a non-vanished ballistic component. Based on the decay of the ballistic component described by the Lambert-Beer law (Eq. 2.10), we can locally assign a scattering mean free path of $12 \mu \mathrm{m}$ to these positions.

Furthermore we qualitatively examined the light transmission of the secondharmonic radiation through the sample. For practical purposes we directly illuminated the sample with monochromatic light at the wavelength $\lambda=405 \mathrm{~nm}$ (close to the SHG wavelength $\lambda=420 \mathrm{~nm}$ ). In transmission, we observed a speckle pattern by eye, which did not show any preference of the forward direction independent of the position of the illuminated spot on the sample. Ideally, a characterization would include a time-resolved transmission measurement, however, the experimental setup (Fig. 3.6) cannot be operated at this wavelength.

In the following we compare these results to calculations of the transport mean free path of comparable systems based on Mie theory [84]. From a standard Mie program we obtained the scattering cross section $\sigma_{\mathrm{s}}$ and the anisotropy parameter $\langle\cos \theta\rangle$ of a single fused silica sphere. Assuming independent scattering and a filling fraction of $f=0.6$ we calculate the transport mean free path for a random medium from monodisperse spheres as a function of sphere radius by Eqs. 2.8 and 2.14. The results from the calculations for both the fundamental radiation $(\lambda=840 \mathrm{~nm})$ and the second-harmonic radiation $(\lambda=420 \mathrm{~nm})$ are plotted in Fig. 6.4. To obtain an estimate for the transport mean free path for our sample we consider the results for sphere radii used for the sample fabrication. The corresponding calculated mean free paths for the fundamental radiation are $l_{\text {Mie }}(125 \mathrm{~nm})=5.5 \mu \mathrm{m}, l_{\text {Mie }}(175 \mathrm{~nm})=3.3 \mu \mathrm{m}$ and $l_{\text {Mie }}(250 \mathrm{~nm})=2.0 \mu \mathrm{m}$. The arithmetic mean is $l_{\text {Mie, } 840 \mathrm{~nm}}=3.5 \mu \mathrm{m}$. For the second harmonic radiation, we obtain $l_{\mathrm{Mie}, 420 \mathrm{~nm}}=0.58 \mu \mathrm{m}$.

From both our sample characterization and the calculations we can conclude two points: firstly, for the fundamental radiation, light transport in the sample is partly 


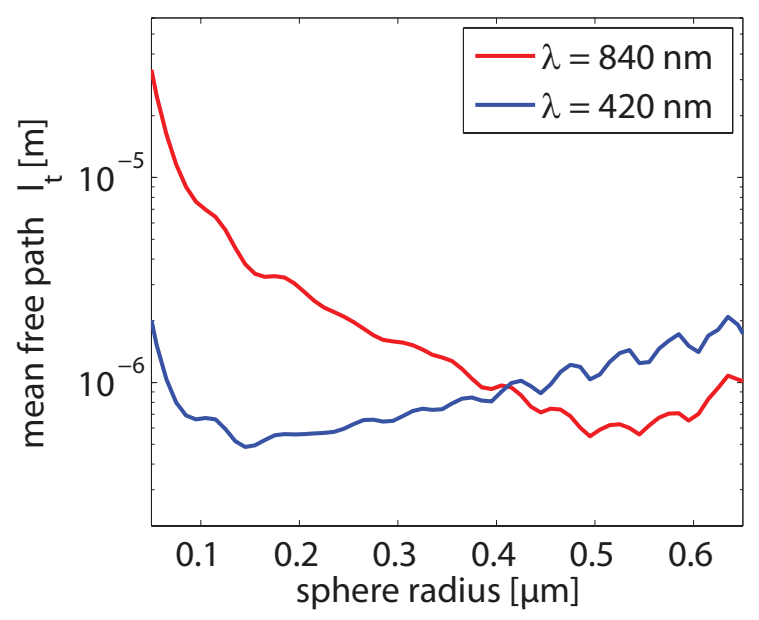

Figure 6.4: Calculated transport mean free path as a function of sphere radius in a disordered slab of monodisperse spheres with a refractive index $n=1.46$ and a filling fraction $f=0.6$.

ballistic and partly diffuse. Secondly, for the SHG, light transport is diffuse. For the fundamental radiation, the ballistic component should be reduced by more than eleven orders of magnitude if a transport mean free path $l=3.5 \mu \mathrm{m}$ would hold over the full slab thickness of $L=85 \mu \mathrm{m}$.

The observed ballistic or weakly scattered transmission can be explained by the presence of cracks inside the medium which are visible at the backside of the sample. The light propagating along these cracks can quickly be transported through the sample with few scattering events. On the other hand, we see that for a considerable fraction of the light the path length is many time the geometrical sample thickness. The long time tail of the decay curve is well described by an exponential decay, as expected from diffusion. We notice that the mean free path obtained from the experiment, $l_{\exp }=3.2 \mu \mathrm{m}$, coincides well with the theoretical results, only deviating by $10 \%$ from the arithmetic mean of the three calculated values. For the secondharmonic radiation we observe only diffusely transmitted light. We also see from Fig. 6.4, that the calculated mean free path for the second-harmonic radiation with $l_{\mathrm{Mie}, 420 \mathrm{~nm}}=0.58 \mu \mathrm{m}$ is considerable shorter in the same range of radii. Although the SHG radiation should be in the same way affected by scattering along cracks, much thinner compact scattering layers along the scattering path are needed to randomize the direction of the light compared to the fundamental radiation.

\subsubsection{Experimental procedure}

\section{Sample positioning}

The sample was mounted on a manually controlled 3-axis stage. The z-position (along the optical axis) was fixed such that air-silica interface is a Fourier plane to the SLM surface; i.e., a flat phase pattern on the SLM results in a tightly focused illumination. While collecting the light in the transmission configuration, the sample was translated with the x-actuator and y-actuator in search for a position at which a spurious SHG signal was detected by the PMT. We performed two series of the experiment at two 
different locations, marked by the dashed circles Fig. 6.3a. For both series we selected a position at which no cracks were visible in the illuminated area of the silica layer. For the second series, the center point of illumination was approximately $1 \mathrm{~mm}$ distant from the edge; for the first series, we carefully determined the position for comparison with the time-resolved transmission measurements (Fig. 6.3c).

\section{Wavefront shaping experiment}

During the experiment, the wavefront was modulated by the SLM in an iterative closed-loop optimization scheme in order to maximize the SHG signal, which was collected by the objective in transmission and detected on the PMT. The segmentation of the wavefront chosen for optimization was successively increased during the experiment $(N=12,48,192,768$ and 19200 segments on the SLM surface). For each level of segmentation, we optimized the signal until convergence was reached. For the optimization scheme, we opted for the so-called 'partitioning algorithm', during which all segments of the SLM are addressed simultaneously [147]. During each iteration of the algorithm, half of the segments are randomly chosen to be static. The other half is synchronously altered by an additional phase shift, in $N_{\varphi}=8$ discrete steps. The concerted modulation provides a high signal-to-noise ratio (SNR), which is beneficial in view of the low photon count rate encountered in this experiment. The feedback signal is fitted with a cosine squared function to determine the best phase value. We observed that the partitioning algorithm has a small drawback, similarly to the observations by Vellekoop et al. in a linear WFS experiment [147]: In rare cases the cosine fit fails to return the best phase value. When the determined optimal phase has a large error, the constructive interference can be almost completely destroyed in a single iteration. Following the suggestion of Vellekoop et al. [147], we solved this problem by keeping the previous SLM phase pattern in memory. After the new iteration, the determined optimized phase pattern is tested in a single feedback measurement and reverted to the previous pattern in the case that the signal has decreased. However, we found that this strategy has the disadvantage that the SNR of a single feedback measurement is lower than the SNR of the phase shift in $N_{\varphi}$ steps. The safety step slows down the optimization when it approaches a maximum, since better phase patterns are likely to be rejected due to noise.

\section{Autocorrelation measurement}

Before and after the WFS experiments, we performed an autocorrelation measurement [108] to obtain information about the pulse duration at the nanocrystals inside the medium. For this purpose, the movable-arm of the Michelson-interferometer was scanned in discrete two-step movement (see 5.2) while the SHG count-rate on the PMT was recorded. 


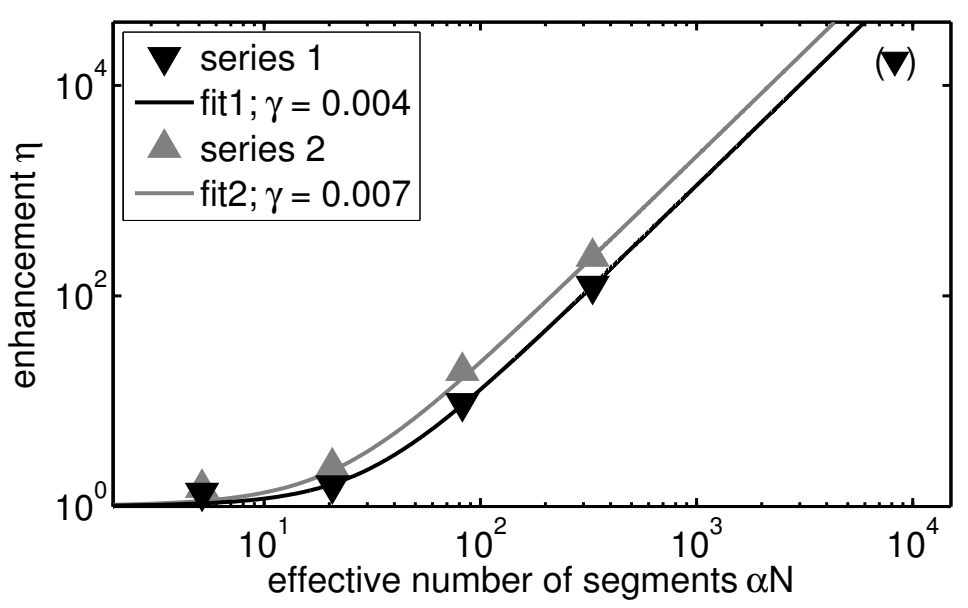

Figure 6.5: Enhancement of the detected SHG count rate $\eta$ versus effective number of segments $\alpha N$. The data points of series 1 correspond to the experiment which we conducted at the location 1 in Fig. 6.3a and which is analyzed extensively in this chapter. The data of series 2 has been acquired in an analogous experiment performed at location 2. The data has been fitted using Eq. 6.3, with the control factor $\gamma$ as fit parameter. For the highest achieved enhancement of series 1 (data point in brackets; $\eta=1.7 \cdot 10^{4}$ ) we aborted the experiment algorithm before convergence was reached; therefore the data point is not considered for the fit. The relative statistical measurement errors due to noise are below 0.01 , since we averaged the count rates over many measurements, therefore we omit showing error bars.

\subsection{Results}

\section{Enhancement}

We found that the wavefront shaping optimization enhances the SHG signal by more than four orders of magnitude (see Figure 6.5). We quantified the enhancement $\eta$ defined by

$$
\eta \equiv \frac{I_{2 \omega, \mathrm{opt}}}{I_{2 \omega, \mathrm{av}}}
$$

where $I_{2 \omega, \text { opt }}$ is the count rate after optimization. As reference, we obtained the average count rate $I_{2 \omega, \text { av }}$ for each level of segmentation by averaging over 200 separate measurements, each acquired with a different random wavefront incident on the sample. Figure 6.5 shows the enhancement as a function of segmentation. An enhancement as high as $\eta=1.7 \cdot 10^{4}$ was reached for the highest segmentation. In this case the algorithm was stopped due to time limitations after three days before convergence was reached. We fitted the data points obtained from the wavefront shaping experiments of the two series by Eq. 6.3. We determined the factor $\alpha \equiv\langle|A|\rangle^{2} /\left\langle|A|^{2}\right\rangle=0.43$ from the amplitude distribution of the incident beam on the spatial light modulator. In the following, we present the results and the analysis of the camera images and the autocorrelation measurements. As results from series 2 do not lead to different conclusions, we only show those for series 1 . 

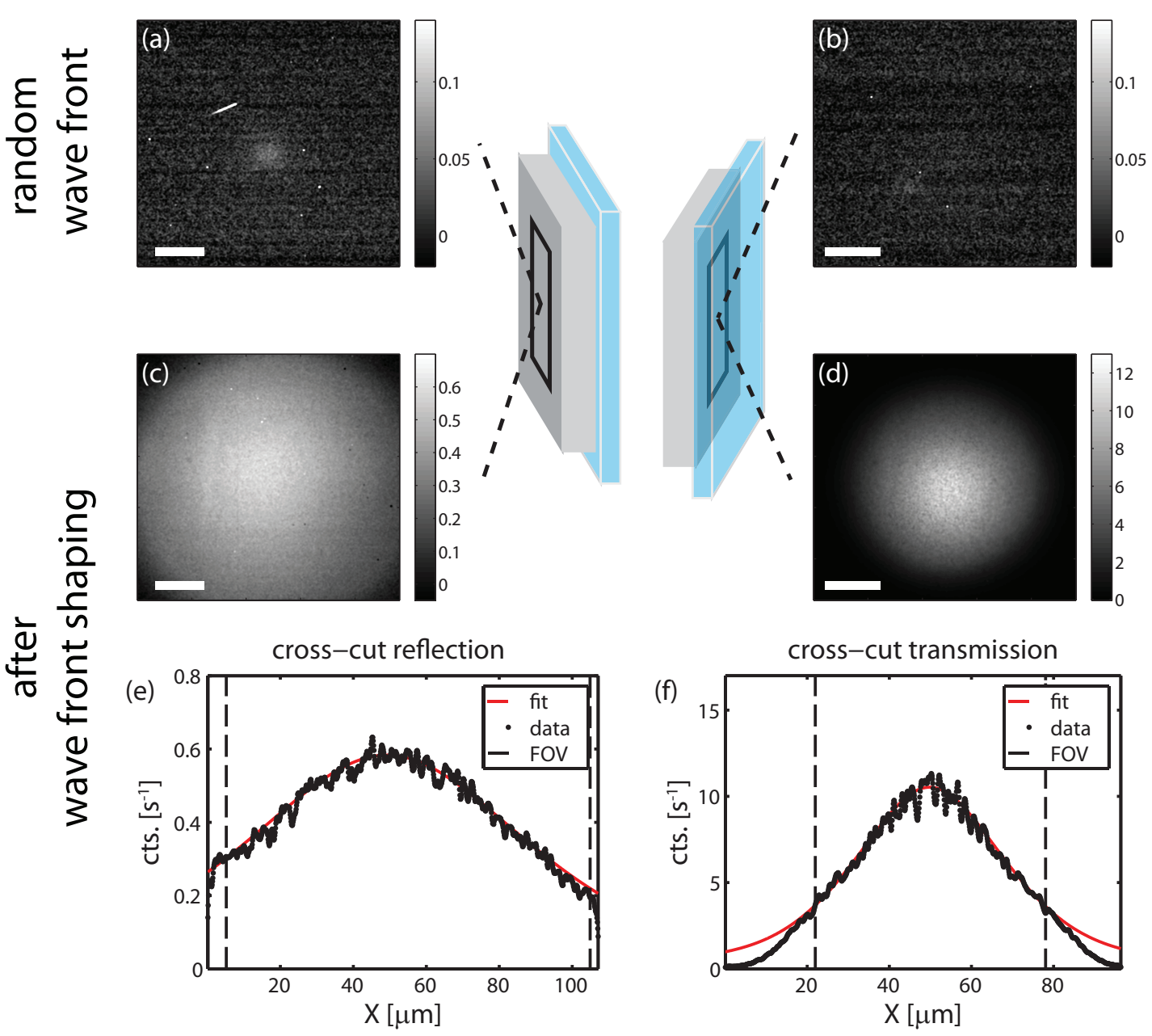

Figure 6.6: Camera images of the second harmonic radiation $(\lambda=420 \mathrm{~nm})$ at the surface of the scattering medium. The scale bars correspond to a length of $20 \mu \mathrm{m}$. (a,b) Average images from 100 measurements with different randomly generated wavefronts, at the silica-air interface (a) and the silica-glass interface (b). (c,d) Images after optimization by wavefront shaping at the front interface (c) and the back interface (d). (e,f) Cross cuts along the horizontal axis through the point of maximum intensity of the diffuse spots. We determine the depth of the nanocrystal in the focus by fitting the diffuse spots within the field of view (FOV) with diffusion theory (red lines). Note that we applied a spatial low pass filter to all images in Fig. 6.6 to remove camera noise; this also slightly reduces the speckle contrast in the images but furthermore has no effect on the further analysis. 

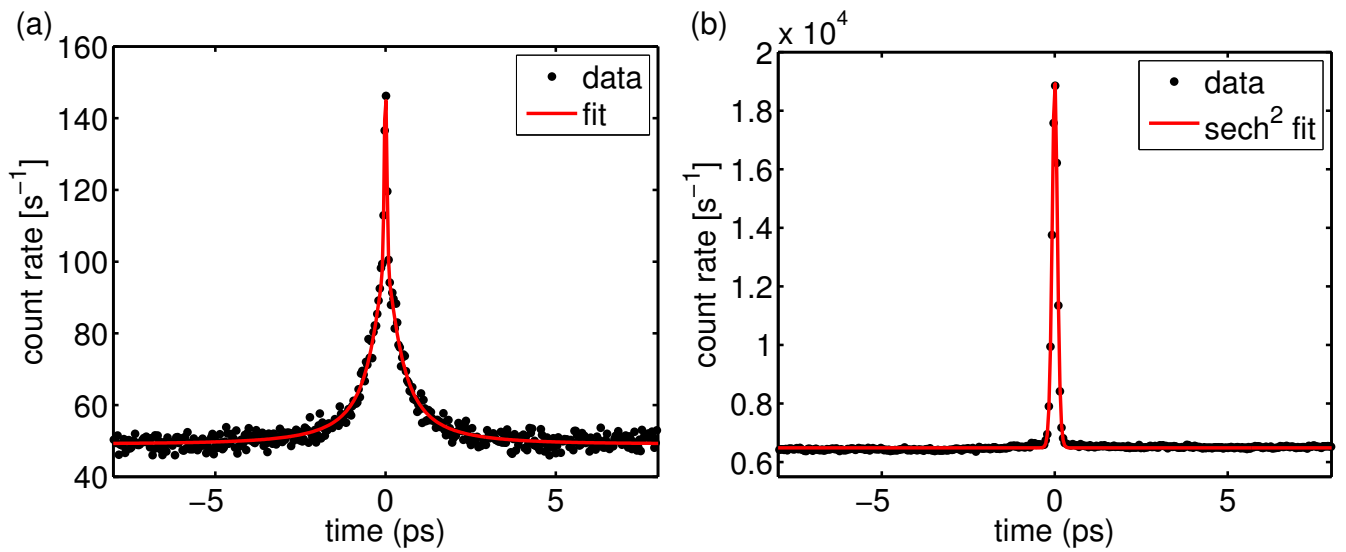

Figure 6.7: (a) Autocorrelation measurement before wavefront shaping. The measurement was performed with a random wavefront applied on the SLM with a low segmentation ( $N=768$ segments). Red line: fit of Eq. 5.2 to the data, where the diffuse intensity propagator (Eq. 2.18) was used to obtain the intensity $\left\langle I_{d}(t)\right\rangle$. The only fit parameter was the particle depth from the silica-air interface, obtaining $z_{1}=28 \mu \mathrm{m}$. (b) Autocorrelation measurement after wavefront shaping optimization with $N=768$ segments. The light is focused in a short pulse. From the full width at half maximum of the $\operatorname{sech}^{2}$ fit $(\mathrm{FWHM}=$ $178 \mathrm{fs}$ ), we determine a pulse duration of $\tau_{\text {focus }}=116 \mathrm{fs}$ (deconvolution factor 0.65 ).

\section{Second harmonic radiation imaged at front and back interface}

Before wavefront shaping, no SHG signal from the nanocrystals can be identified from the camera measurements (see Fig. 6.6). Each of the shown images is an average of 100 measurements, which have been separately acquired each with a different random incident wavefront. In reflection, at the position of the incident fundamental radiation, a faint reflection at the second harmonic frequency is visible. This signal is caused by second harmonic generation in the silica host beads. It can be suppressed to the largest extent when a non-flat wavefront is applied on the SLM such that a tight focus is avoided. In transmission, no signal is visible at all before optimization.

After wavefront shaping, a symmetric diffuse spot of SHG radiation can be clearly observed from both interfaces. We found that the sharpest image of the diffuse spot in transmission is obtained when the focal plane of the objective coincides with the sample interface. Translating the focal plane of the second objective towards the sample did not lead to a sharp (or partly sharp) image of the nanocrystal, proving that the light transport of the SHG radiation is diffuse.

We determined the depth of the nanocrystal inside the slab by fitting the diffuse spots with diffusion theory [14]. For the fits we used the slab thickness L = $(85 \pm 10) \mu m$ as determined by bright field microscopy (Fig. 6.3a), and we used the nanocrystal depth as fit parameter. From the reflection image, we obtain the particle distance to the interface of $z_{1}=58.5 \mu \mathrm{m}$. From the transmission image we obtain $z_{2}=20.5 \mu \mathrm{m}$. The error margin for the fits is about one transport mean free path [14], which for the SHG light is below $2 \mu \mathrm{m}$. The sum $z_{1}+z_{2}=79 \mu \mathrm{m}$ coincides with the slab thickness $L$ with the accuracy of the thickness measurement. 


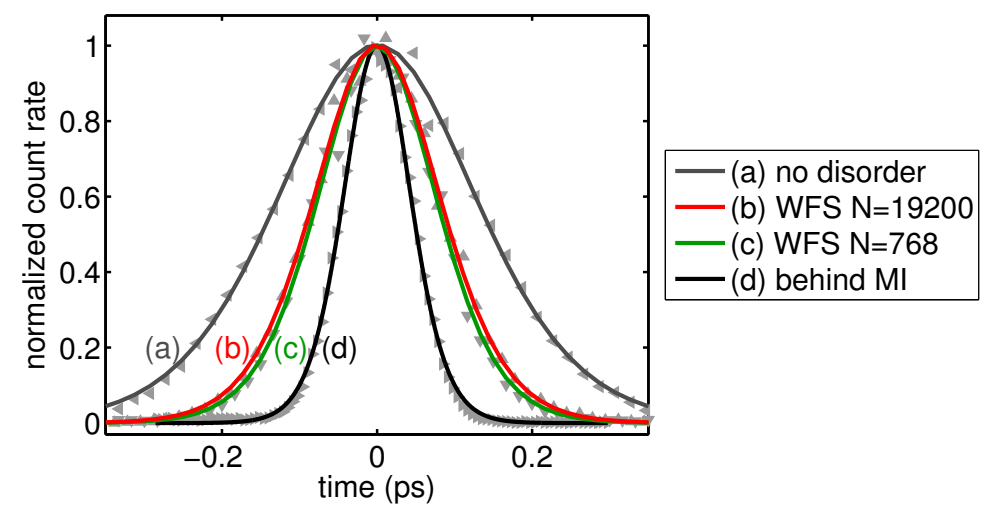

Figure 6.8: Normalized autocorrelation measurements. Data points (grey triangles) are plotted together with the corresponding sech ${ }^{2}$-fits. (a) At the sample position without a disordered layer. Full width at half mean (FWHM) is $298 \mathrm{fs}$. (b) After the WFS experiment with $\mathrm{N}=19200$ segments; FWHM is 190 fs. (c) After the WFS experiment with $\mathrm{N}=768$ segments; FWHM is 179 fs. (d) At the output of the Michelson interferometer; FWHM is $102 \mathrm{fs}$.

\section{Autocorrelation measurement before and after WFS}

Figure 6.7 shows the autocorrelation measurements before wavefront shaping and after the optimization with $N=768$ segments. The measurement before the optimization was performed with a random wavefront applied on the SLM with the same segmentation into $N=768$ segments. By this, we ensure that the second harmonic generation on silica particles at the interface is negligible and we only detect SHG from particles inside the medium. The result shown in Fig. 6.7 is typical for the autocorrelation of a speckle pulse: Around zero time delay, we see a sharp peak (3:1 peak-background ratio) resulting from the autocorrelation of each of the temporal subpulses contained in the speckle pulse. At larger time delays we observe the crosscorrelation of the subpulses, decaying on a time-scale on the order of picoseconds. In Sec. 5.3.1 in the previous chapter we have described this behaviour in more detail.

Here, the autocorrelation shows hardly any randomness on the wings, in contrast to what could be expected from the autocorrelation of a speckle pulse in a single mode. This result indicates that several nanocrystals are present in the probed sample volume and that the particles support several independent modes for the SHG process, resulting in averaging over speckle. The fact that the transition from the short-term correlation to the wings is clearly visible at a 2:1 signal ratio on top of the background indicates that for the incident wavefront only diffuse light reaches the nanocrystal(s). We will discuss the fit to the data in the discussion section.

After wavefront shaping, we observed a narrow autocorrelation peak, indicating that a short pulse is formed inside the medium (Figure 6.7b). From the full width at half maximum of the $\operatorname{sech}^{2}$ fit $(\mathrm{FWHM}=178 \mathrm{fs})$, we determined a pulse duration of $\tau_{\text {focus }}=116$ fs (deconvolution factor 0.65 ).

We compare the pulse duration after the wavefront shaping experiments with the output pulses of the laser and the situation before wavefront shaping. The comparison is summarized in Fig. 6.8. At the output of the Michelson interferometer, the pulses have a duration of about $\tau_{\text {laser }}=66 \mathrm{fs}$, determined from Fig. $6.8 \mathrm{~d}$ with a deconvolution 
(a)

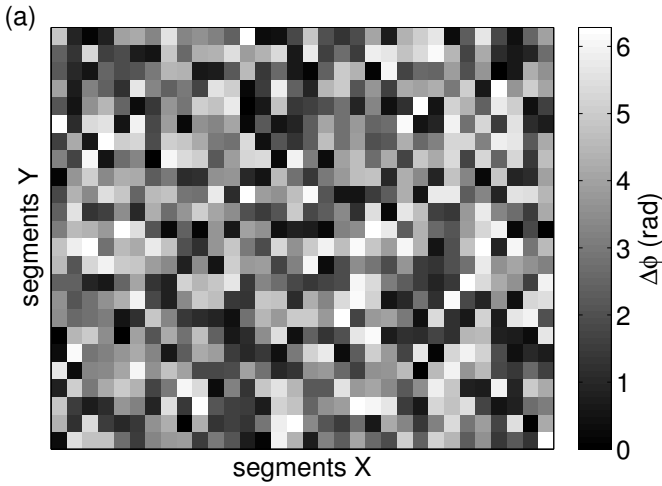

(b)

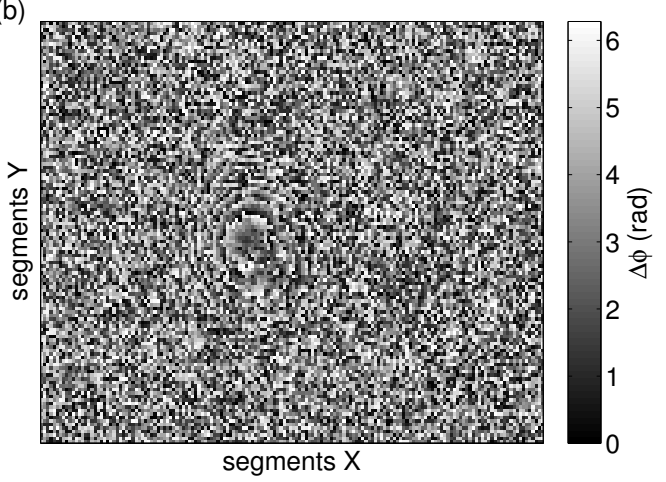

Figure 6.9: Phase pattern applied on the SLM after the wavefront shaping optimization for with a segmentation into $N=768$ (a) and $N=19200$ (b) independent segments. For the lower segmentation, no correlation between adjacent segments is visible. For the higher segmentation, concentric rings are visible, and correlations with neighboring segments. They indicate the forming of a Fresnel lens by which the focus is translated from the front interface into the medium.

factor 0.65. Fig. 6.8a shows the autocorrelation at the sample position. For this measurement, we placed single $\mathrm{BaTiO}_{3}$ nanocrystals on a bare glass substrate without a disordered medium. Since the light travels through a number of optical elements from the Michelson interferometer to the sample, the pulses are stretched due to material dispersion to a duration of $\tau_{\text {chirp }}=193$ fs (see Fig. 6.8a). The focused pulses are shorter again in duration, $\tau_{\text {focus }}=116$ fs when optimized with $N=768$ segments (Fig. 6.8c) and $\tau_{\text {focus }}=123$ fs when optimized with $N=19200$ segments (Fig. 6.8c). The highly segmented wavefront leads to a longer pulse duration. This interesting trend will be discussed and put in a broader scope in the discussion section.

\section{Optimized phase front and projection onto sample}

The optimized phase pattern is random for a low segmentation (Fig. 6.9a) and shows structure and correlations for a high segmentation (Fig. 6.9b). For the lower segmentation, no correlation between adjacent segments is visible. For the higher segmentation, concentric rings are visible, and correlations with neighboring segments. The observation indicates the forming of a Fresnel lens by which the focus is translated from the front interface into the medium and that the focus is partly formed by ballistic light.

To understand this result we image the propagating wavefront at the fundamental frequency for the same two phase settings of the SLM without a scattering medium in order to identify a ballistic contribution to the focus on the nanocrystals. Equivalently to the sample placement procedure, we placed a bare glass cover slip at the sample position, such that its front interface is a Fourier plane of the SLM surface. With the oil-immersion objective on the transmission side, we image the wavefront at different depths inside the cover slip. The resulting images are shown in Fig. 6.10. For the series of Fig. 6.10a, a random wavefront (segmentation $N=19200$ ) was projected onto the sample. For the latter case a speckle pattern is visible at all depths, with one exception: at the substrate surface a tight focus is visible at the center, originating 

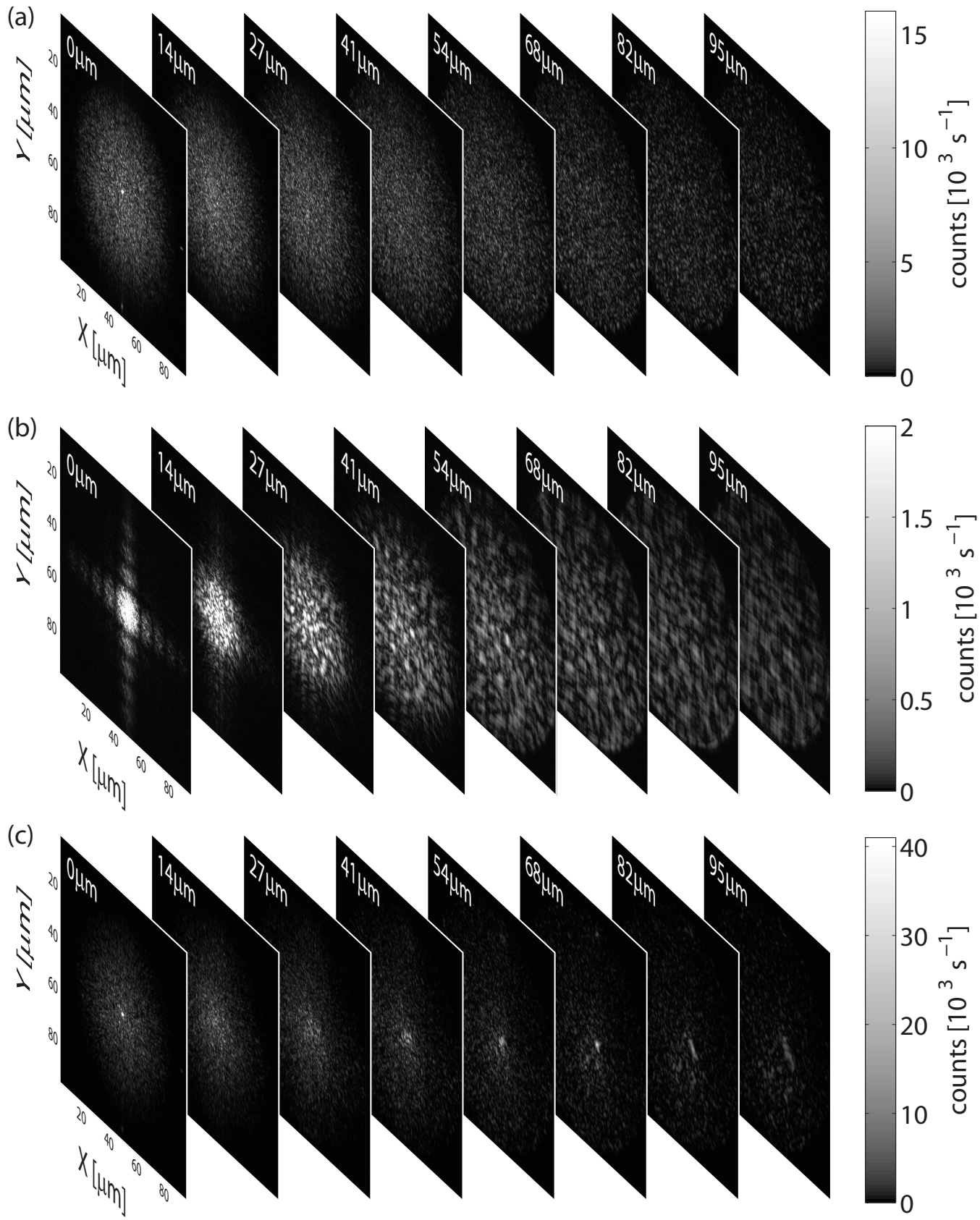

Figure 6.10: Propagation of different wavefronts at the fundamental wavelength $(\lambda=$ $840 \mathrm{~nm}$ ) in a bare glass substrate. The position of the image along the optical axis is indicated in the images. (a) A random wavefront (segmentation $N=19200$ ) is projected onto the sample. (b) The wavefront resulting from the wavefront shaping experiment with $N=768$ segments is incident. (c) The wavefront resulting from the wavefront shaping experiment with the highest segmentation $(N=19200)$ is incident. 
from light which is not modulated by the SLM: it is caused by partial reflection $(\approx 5 \%)$ of the beam at the front window of the SLM. For the series of Fig. 6.10b, the wavefront resulting from the experiment with $N=768$ segments was incident. At the interface the superposition of the Fourier transform of a single SLM segment and the entire random wavefront on the SLM is visible. The wavefront diverges as it propagates deeper into the medium. The scale bar was adjusted such that the lower intensities at larger depths are well-displayed; the count rate in high intensity areas in the first images is beyond the maximum of the scale bar $2 \cdot 10^{3} \mathrm{~Hz}$. The overall count rates of the series was lower compared to the two other series since we placed an additional neutral density filter in front of the camera. In the last series of Fig. 6.10c, the wavefront resulting from the experiment with the highest segmentation $(N=19200)$ was incident. At the objective position $z_{\mathrm{obj}}=68 \mu \mathrm{m}$, a focus was formed. We observed that the light focused deeper inside the glass substrate than the determined depth of the nanocrystal inside the disordered medium (see Fig. 6.6). This difference can be consistently explained by comparing the refractive indexes of both media in view of Snell's law. The focus resulting from a converging wavefront is translated deeper into the medium as the refractive index increases. Since the refractive index of the glass cover slip is higher than the effective index of the medium, the focus manifests at a larger depth.

\subsection{Discussion}

\section{Spatial and temporal focus}

Our experimental results strongly support the picture that we achieved focusing of a short pulse onto a single point deep inside the random medium. Regarding the temporal aspect, the autocorrelation measurement clearly proves that at the point of the SHG conversion a short pulse is present (Fig.6.7). A more complex temporal distribution, e.g., the formation of a double pulse, would reflect in an autocorrelation of higher complexity. However, based on the autocorrelation measurement alone we are not able to distinguish the situation of a single spatiotemporal focus from possible several separate spatiotemporal foci. Both situations would result in a narrow autocorrelation.

Regarding the spatial aspect of the focus, we consider the images of the diffuse spots on the sample front and back interface. The spots are center-symmetric, which would not be the case for two laterally separated light sources. Furthermore, the analysis of the diffuse spots at both interfaces consistently give the same result for the position of the light source along the optical axis, with an accuracy on the order of the transport mean free path. We can therefore state that all the enhanced SHG light originates from a small volume inside the medium of the dimensions of the transport mean free path. Within this volume, the light forms a short pulse.

\section{Transition from focus of diffuse light to focus of ballistic light}

Our results indicate that both diffuse light and ballistic light can play a role in creating the focus. For the optimization by $N=768$ segments the diffuse component 
predominates by far and we do not see indications that a ballistic contribution is optimized. For the higher segmentation $N=19200$ both the diffuse and the ballistic component contribute a relevant fraction to the focused light.

The observation that the optimized pulses are shorter than the chirped incident pulses $\left(\tau_{\text {chirp }}=193\right.$ fs vs. $\tau_{\text {focus }}=116$ fs for $N=768$ and $\tau_{\text {focus }}=123$ fs for $\left.N=19200\right)$ can only be explained by the contribution of multiply scattered light. There are two ways to shorten a strongly chirped pulse; first, dispersion compensation, and second, to a limited extent, spectral selection. The latter effect can only shorten pulses which are strongly chirped, i.e., when the red and the blue end of the spectrum are temporally separated by more than the original pulse duration, which is the case here $\left(\tau_{\text {chirp }}=193 \mathrm{fs}\right.$ vs. $\left.\tau_{\text {laser }}=66 \mathrm{fs}\right)$. Focusing through a random medium by shortpulse WFS inherently performs a combination of both dispersion compensation and spectral selection as was shown in chapter 3. However, short-pulse WFS will only lead to any of the two effects if the shaped scattered fields are sufficiently random. In other terms, the $C^{(1)}$-frequency correlation (Eq. 2.31) needs to be significantly narrower than the width of the spectrum, which implies multiple scattering.

All evidence suggests that the pulse obtained by the $N=768$ optimization is only formed from diffuse light. The optimal phase pattern is random (Fig. 6.9a) and the propagation of optimized wavefront in the glass substrate does not show a focusing effect. As we will see in the next section, the enhancement is well-described based on the diffusion model. In addition to these experimental findings, the resulting pulse duration $\tau_{\text {focus }}=116 \mathrm{fs}$ exactly coincides with the most frequently obtained pulse duration in the simulation in chapter 4 . In this simulation, we use a pulse with the same initial pulse duration $\tau_{\text {laser }}$ and the scattering is modeled by diffuse transport (see Fig. 4.4).

For the optimization with a segmentation of $N=19200$ we observe clear signs for an additional contribution of ballistic light. A part of the phase pattern exhibits Fresnel rings (Fig. 6.9b) and the projection of the wavefront into a homogeneous medium leads to a tight focus at the correct depth (Fig. 6.10c). The pulse duration $\tau_{\text {focus }}=123 \mathrm{fs}$ is enhanced compared to the lower segmentation case, but nevertheless remains significantly below the duration of the chirped input pulses. This difference indicates that the focus is still formed to the largest fraction by multiply scattered light. If the focus would be constructed only from the ballistic light, the autocorrelation of the focus would reproduce exactly the result from the chirp measurement. As this optimization was stopped before convergence we cannot state whether, or to which degree, this mixed contribution is stable. This is an interesting question which might be addressed in future studies.

The different behaviour of the low segmentation and high segmentation optimization can be explained by Fig. 6.9. The radial distance between two concentric rings forming the Fresnel lens in Fig. 6.9b is shorter than the segment size in Fig. 6.9a, i.e., with the lower segmentation the ballistic focus cannot be formed and only multiply scattered light is optimized. Therefore the choice of segmentation resolution allows us to selectively choose either to optimize purely diffuse light or to include the ballistic component in the optimization. 


\section{Enhancement and segmentation}

As Fig. 6.3 illustrates, the relation between enhancement $\eta$ and segmentation $N$ is well-described by the fit by Eq. 6.3 with exception of the data points at the lowest segmentation. There the experimentally observed enhancement was above the fit by Eq. 6.3, which we can explain in the following way. In the model leading to equation 6.3 we assume that the focus is converged to a single pulse focused in a single coherent volume in one of the nanocrystals. From all other SHG sources outside this volume (and at earlier and later arrival times), we assume a contribution given by the ensemble average. However, for a low number of controlled segments, the highest feedback signal may result from optimizing two or more of these 'spatiotemporal focal points'. Consequently a higher enhancement should be observed than predicted by the model. For an increasing segmentation the focus quickly converges to a single spatiotemporal focus and the model gives an accurate description of the scaling between $\eta$ and $N$. The good agreement in the range from $N=48$ to $N=786$ furthermore supports the interpretation that the focus formed by randomly scattered light.

Based on the control factor $\gamma=0.004$ obtained from the fit in Fig. 6.5 with Eq. 6.5 we can approximate the number of competing spatiotemporal coordinates. We will use the factors $\gamma_{\alpha}=0.28$ and $\gamma_{r}=0.57$ which were calculated for equally sized $\mathrm{BaTiO}_{3}$-nanocrystals in in the previous chapter (Sec. 5.3.2). This leaves $\gamma_{n} \gamma_{\tau}=$ $0.025=(40)^{-1}$ as the value of the product of the remaining two factors, i.e., the inverse product of the number of nanocrystals in the probe volume and the number of temporal degrees of freedom. We determine $\gamma_{\tau}$ by fitting Eq. 5.2 to the autocorrelation before wavefront shaping (Fig. 6.7a). Here the obtained intensity $\langle I(t)\rangle$ is averaged over all particles in the probed volume, in contrast to the experiment described in the previous chapter where only a single particle was detected. Evaluation of Eq. 5.9 with $\left\langle I_{d}(t)\right\rangle$ gives $\gamma_{\tau}=0.15$. Based on this calculation $\left(\gamma_{n}\right)^{-1}=40 \gamma_{\tau} \approx$ 7 nanocrystals are contributing to the SHG signal. This number differs from an estimate based of the mass fraction of nanocrystals inserted in the slab: Assuming a homogeneously distributed diffuse fundamental intensity in the probed volume of the slab, we expect approximately 30 to 50 contributing particles. The deviation indicates that the assumption of homogeneous diffuse transport is not valid for the sample. The obtained average particle depth from the fitted autocorrelation ((Fig. 6.7a)) further supports this conclusion: The fit based on diffusive propagation with a single diffusion constant gives an average particle depth $z_{0}=28 \mu m$, contradicting the SHG nanocrystals are distributed in the first layer on top of the substrate, $50 \mu \mathrm{m}$ to $80 \mu \mathrm{m}$ away from the injection point of the fundamental pulses.

\subsection{Conclusions}

In summary, we have experimentally demonstrated spatiotemporal focusing on nanocrystals hidden deep inside a disordered medium by wavefront shaping using secondharmonic generation from embedded nanocrystals. Our data strongly supports the picture that a single spatiotemporal focus is formed inside the medium. In the investigated system, light propagation of the fundamental radiation is in the regime between ballistic and diffuse transport; for the SHG radiation, light transport is diffu- 
sive. Without optimization, light transport to the nanocrystals is largely dominated by diffusion. By means of the segmentation of the wavefront we can control whether the optimized focus is formed only from multiply scattered light or both from ballistic and randomly scattered light. In the diffuse regime, the observed enhancement of the SHG signal agrees with our theory. To conclude, we demonstrated that our approach for spatiotemporal focusing inside complex photonics systems is feasible, which opens the door for many experiments not only in disordered systems, but also metamaterials or photonic crystals. Furthermore, the studied experimental system in particular is evidently interesting to study the transition regime between ballistic and diffuse light transport. 


\section{Optimal spatiotemporal focusing through random media}

We present a new approach to achieve optimal spatiotemporal focusing through complex scattering media. In contrast to the preceding chapters, the scheme is based on a wavefront synthesizer which can modulate waves both spatially and temporally. Using a nonlinear feedback signal to iteratively shape the incident pulsed wavefront, we show that the limit of a spatiotemporal matched filter can be achieved, i.e., the wave amplitude at the intended time and focus position is maximized for a given input energy. The method is successfully tested by simulations based on a experimentally acquired ultrasound transmission matrix and is generally applicable to all types of waves.

\subsection{Introduction}

Most imaging systems are based on the ability to focus a wave beam inside the area of interest. In the context of echographic imaging, focusing of ultrasound in the human body can be achieved with a transducer array and electronic delay lines: the same pulsed waveform is sent from each transducer with the appropriate delay making all the waveforms converge in synchrony at the desired focus location. However, that principle is not practicable any more as soon as the sample thickness becomes larger than the mean free path, i.e., the average distance between two random scattering events. In such a strongly scattering medium, it has been shown with ultrasonic waves that spatiotemporal focusing can be achieved using time reversal: a training pulse is

The content of this chapter has been published as: J. Aulbach, A. Bretagne, M. Fink, M. Tanter, and A. Tourin, Phys. Rev. E 85016605 (2012). 
first sent from a source located at the intended focal point, travels through the scattering medium and is captured at a transducer array, the 'time reversal mirror' [39]. The waveforms received on the time reversal mirror are flipped in time and sent back, resulting in a wave converging at the desired focus location. Time reversal focusing is optimal in the sense that it achieves a spatiotemporal matched filter, a term wellknown from signal processing; for a given input energy, the amplitude of the pulse at the focal spot is maximal [156]. Time reversal has also been implemented for microwaves [42]. For optical waves, time reversal has not been demonstrated yet, since optical time reversal mirrors are challenging to realize.

Therefore in optics a different approach, named 'wavefront shaping' (WFS), has been taken to steer light through strongly scattering media [13]. The principle is based on spatial phase modulation by Spatial Light Modulators (SLM), which enables one to modulate the complex amplitude of the independent modes of the incident wavefront. The intensity in the selected output mode, e.g., the focal spot, is used to match the wavefront to the scattering medium by an adaptive algorithm. Owing to their enormous amount of pixels, state-of-the-art spatial light modulators allow achieving transmission of substantial amounts of the input energy [14].

A big advantage of adaptive wavefront shaping approaches lies in the fact that a direct access to the amplitude at the focus is not required, such that any type of intensity probe can be used. For instance the fluorescence from dye molecules allows to focus on objects hidden inside a complex medium [14]. The concept has been applied to other types of waves, such as surface waves [31] and ultrasonic waves in the single scattering regime for medical imaging [157] and MR-guided ultrasonic therapy [158].

Initially introduced in the monochromatic regime, WFS has recently been extended to broadband light for spatiotemporal focusing [135]. In this work it was shown that spatial control of the input waves is sufficient to achieve spatiotemporal control of the scattered waves. The key idea is to use coherence gating for optimizing a single specific predetermined point in time. Performing the optimization at a specific time in the speckle amounts to combine all the scattering paths that have about the same length. This principle also works efficiently without defining a focusing time in the case when the temporal delay spread induced by the scattering medium is comparable to the input pulse duration [109]. In both cases, the missing control of temporal degrees of freedom is compensated by manipulating spatial ones. In a complementary way, temporal control of the input light based on pulse shaping [59] can be used to achieve spatiotemporal focusing by spatially localized phase compensation [159]. Both approaches exploit the fact that temporal and spatial degrees of freedom are mutually convertible in a complex medium [100].

In the present chapter, we demonstrate how to fully control both spatial and temporal degrees of freedom to achieve optimal spatiotemporal focusing for a broadband ultrasonic wave propagating through a complex multiple scattering medium. Like in the context of time reversal focusing, we use the term 'optimal' in the sense of a spatiotemporal matched filter, i.e., for a given input energy, our method maximizes the wave amplitude at the intended time and focus position. The difference with time reversal is that no amplitude measurement is required at the focus location. Instead spatial and frequency resolved wavefront shaping can be achieved with a 
(a)

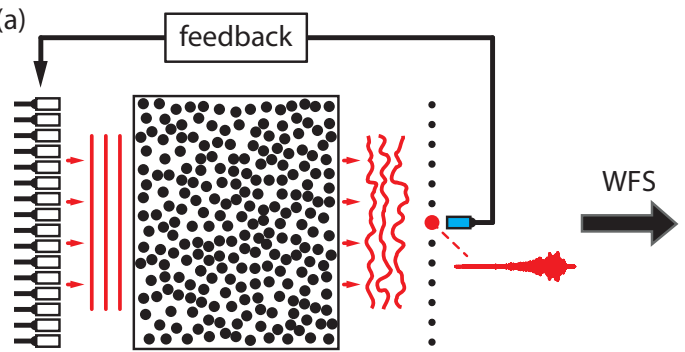

(b)

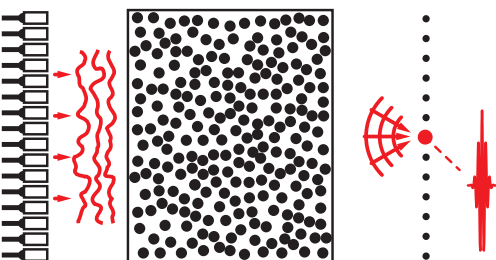

Figure 7.1: Spatiotemporal focusing by wavefront shaping with a nonlinear feedback signal.

nonlinear feedback intensity signal and is capable to reach optimal spatiotemporal focusing. Thus our concept should be generalizable to all types of waves.

The chapter is structured as follows. In the first part we provide an illustration of our experimental scheme, followed by a review of the definition of optimal focusing in terms of a spatiotemporal matched filter and a detailed description of the wavefront shaping optimization. In the second part, we demonstrate by simulations that our scheme leads to optimal spatiotemporal focusing. The simulations are based on the experimental data of the linear transmission of a random medium. After having described the measurements of the transmission matrix, we present the results of the simulations and confirm the optimal focusing by comparison with a simulated time reversal experiment.

\subsection{Wavefront shaping with nonlinear feedback for optimal spatiotemporal focusing}

\subsubsection{Concept}

In Fig. 7.1 the experimental system and the principle of our idea are illustrated. Our aim is to achieve optimal spatiotemporal focusing of a broadband signal through a thick random scattering medium without requiring a measurement of the field amplitude at the focal point. For this purpose we propose the following scheme. A transducer array is placed in front of the medium which can synthesize arbitrary signals over the considered bandwidth. We place a time-integrating nonlinear detector at the focus point in the receiver plane. Using the detector response for a suitable optimization scheme, we find the signal which is to be emitted from the transducer array to achieve optimal focusing.

\subsubsection{The matched filter approach for optimal focusing}

Our goal is to maximize the signal amplitude at some focus point $m_{0}$ in the receiver plane at time $t_{0}$ for a given energy radiated from $N$ points in the emitter plane. In signal processing, this is known as the spatiotemporal matched filter approach. 


\section{Spatial matched filter}

At first we recapitulate the conditions for optimal spatial focusing in the sense of a spatial matched filter[156]. Let $(E(\omega))_{n}, 1 \leqq n \leqq N$ be the input signals in the emitter plane, and correspondingly $(F(\omega))_{m}$ the signals received in the image plane. The propagation through the medium from the emitter points to the control points at one frequency is described by:

$$
F(\omega)=\mathbf{T}(\omega) E(\omega)
$$

where $\mathbf{T}(\omega)$ is the propagation operator, or transfer matrix, between the points at a given frequency $\omega$. The amplitude received at the focal point $m_{0}$ at a single frequency $\omega$ is given by

$$
F_{m_{0}}(E)=\frac{\left\langle\mathbf{T} E \mid F^{(0)}\right\rangle}{\|E\|}=\frac{\left\langle\left. E\right|^{t} \mathbf{T}^{*} F^{(0)}\right\rangle}{\|E\|}
$$

where

$$
F^{(0)}=\{0, \ldots, 0,1,0, \ldots, 0\}
$$

is the projection onto $m_{0}$. The inequality of Cauchy-Schwartz sets the upper bound for this expression:

$$
\frac{\left\langle\left. E\right|^{t} \mathbf{T}^{*} F^{(0)}\right\rangle}{\|E\|} \leqq\left\|{ }^{t} \mathbf{T}^{*} F^{(0)}\right\|
$$

Equality holds, if $E \propto^{t} \mathbf{T}^{*} F^{(0)}$, or

$$
\forall n E_{n}(\omega)=\alpha(\omega) T_{m_{0} n}^{*}(\omega) .
$$

The maximum amplitude at the focal point $m_{0}$ is reached when all $E_{n}$ are proportional to the complex conjugate of the respective transmission coefficient. This situation corresponds to a phase conjugation experiment or a time reversal experiment at a single frequency.

\section{Temporal matched filter}

Let us denote by $T_{m_{0} n}(t)$ the impulse response between emitter $n$ and receiver $m_{0}$, which is the Fourier Transform of the respective transfer function in the frequency domain $T_{m_{0} n}(\omega)$. When one aims at maximizing the amplitude received at time $t_{0}=0$, it is well-known that $E_{n}(t)=T_{m_{0} n}(-t)$ has to be sent from $n$. The signal at $m_{0}$ then writes as

$$
F_{m_{0}}(t)=\sum_{n} T_{m_{0} n}(t) \otimes T_{m_{0} n}(-t)=\sum_{n} \int d \omega e^{i \omega t} T_{m_{0} n}(\omega) T_{m_{0} n}^{*}(\omega)
$$

which is the autocorrelation of the impulse response, or the inverse Fourier transform of the power spectrum, respectively. It is an even function with its maximum value at $t=0$ when all frequency contributions add in phase. Hence the magnitude at $t_{0}$ is directly proportional to the transmitted energy. As $T_{m_{0} n}(-t)$ is the Fourier Transform of $T_{m_{0} n}^{*}(\omega)$, it also achieves the spatial matched filter (Eq. 7.5).

This signal exactly corresponds to the one transmitted in a broadband time reversal experiment. In the first step, the control point $m_{0}$ in the image plane behaves 
like a source, such that the wave field recorded by the two-way transducers in the emitter plane is given by $T_{n m_{0}}(t)$. In the second step, the recorded fields are emitted in a time-reversed manner. Under assumption of reciprocity the emission writes as $E_{n}(t)=T_{n m_{0}}(-t)=T_{m_{0} n}(-t)$ and thus the focus is given by Eq. 7.6. Hence, time reversal exactly achieves a spatiotemporal matched filter since it implements a spatial filter at each frequency of the incident pulse and the correct phase relation between the frequency components is intrinsically recovered.

\subsubsection{Detector response in the simulations}

We consider a system with a second-order nonlinearity, which could be realized, e.g., by a nonlinear scatterer, such as microbubbles in acoustics [160, 161] or nonlinear nanocrystals [141] in optics, combined with a time-integrated detection of the scattered second-order response. We calculate the detector response by

$$
J^{S H}=\alpha \int d t\left|F_{m_{0}}^{2}(t)\right|^{2}
$$

where $\alpha$ is the second-order susceptibility and $F_{m_{0}}(t)$ the amplitude at the focus point in the time domain.

In the following we show that the optimization of $J^{S H}$ leads to optimal spatial and temporal focusing of the waves at the detection point. In order to achieve optimal spatiotemporal focusing of a broadband pulse, we perform the wavefront shaping algorithm in two stages, which separately achieves first spatial focusing and then temporal focusing. It is worth noting that for the first phase, to achieve spatial focusing, the detection of the linear intensity would be sufficient. However, for an experimentally convenient approach, we use the nonlinear detector for both spatial and temporal focusing.

\subsubsection{Steps of the wavefront shaping algorithm}

In the following we lay out the wavefront shaping algorithm and prove its performance as a matched filter from a theoretical point of view. In order to achieve optimal spatiotemporal focusing of a broadband pulse, we perform the wavefront shaping algorithm in two stages. During the first phase, we achieve optimal spatial focusing. Based on this result, optimal temporal focusing is achieved after the second phase.

\section{Phase one: spatial focusing}

The direct implementation of wavefront shaping as applied in optics to monochromatic acoustic waves would be described as follows: at first an arbitrary wavefront is sent from the emitter plane. The intensity is then measured at the intended focal point. This can be done directly in measuring the pressure field with a hydrophone or, in the case of a soft elastic medium, in measuring the displacement induced at the focal point by the radiation force, which is proportional to the acoustic intensity [157]. Then the phase of the first transducer is cycled between 0 and $2 \pi$ while the intensity is recorded. The phase value for which intensity at focus is found maximum is then stored in the memory and the same operation is repeated for each transducer. 
Once the optimal phase has been stored for each transducer, the wavefront can be synthesized by exciting each transducer with the determined optimal phase.

Narrowband optimization by the Hadamard method. Instead of using such an optimization scheme element by element, we perform a basis transformation to construct virtual transmitters $\hat{E}_{j}$, defined as linear combinations of real ones, which greatly improves the sensitivity of the method and reduces the number or iterations [157]. The coefficients of the combination are taken as the columns $H_{n}(1<n<$ $N$ ) of an $N$ by $N$ Hadamard matrix $H$, with elements $H_{j n} \epsilon\{-1 ; 1\}$. This choice ensures that the amplitude transmitted from each transducer is maximal. The basis transformation is performed by

$$
\hat{E}=\mathbf{H}^{-1} E
$$

and the inverse transformation by

$$
E=\mathbf{H} \hat{E}
$$

The field in the receiver plane is given by

$$
F=\mathbf{T} E=\mathbf{T H} \hat{E}=\hat{\mathbf{T}} \hat{E}
$$

where $\hat{\mathbf{T}}$ is the transfer matrix between the emitters in the Hadamard basis and the receivers in the canonical basis. For the optimization algorithm, we arbitrarily chose the first column as the reference. $4 N$ coded beams are sent into the scattering medium as:

$$
\begin{gathered}
C_{j}^{(\delta)}=\hat{E}_{\text {ref }}+\hat{E}_{j} e^{i \delta} \\
j \epsilon[1, N] ; \delta \epsilon\left[0, \pi, \frac{\pi}{2},-\frac{\pi}{2}\right]
\end{gathered}
$$

After each transmission the resulting intensity at the focal point $m_{0}$ is given by

$$
I_{j, \delta}=a_{r e f}^{2}+a_{j}^{2}+2 a_{r e f} a_{j} \cos \left(\phi_{r e f}-\phi_{j}-\delta\right),
$$

where $a_{j} e^{i \phi_{j}}=\hat{T}_{m_{0} j}$ is the corresponding transmission coefficient from the transfer matrix $\hat{\mathbf{T}}$. From the set of measured intensities we are now able to calculate the optimal wavefront. The complex amplitude to be transmitted from each virtual transducer to optimize the intensity is given by the following expressions:

$$
\hat{E}_{j}^{s m f}=a_{j}^{s m f} e^{i \phi_{j}^{s m f}}\left\{\begin{array}{c}
a_{j}^{s m f}=a_{j}=\sqrt{I_{j, 0}+I_{j, \pi}-\frac{I_{r e f, 0}}{2}} \\
\phi_{j}^{s m f}=\phi_{r e f}-\phi_{j}=\arctan \left(\frac{I_{j, \pi / 2}-I_{j,-\pi / 2}}{I_{j, 0}-j_{n, \pi}}\right)
\end{array}\right.
$$

where the $\phi_{j}^{s m f}$ compensate for the phase differences between the contributions at the focal point. The choice of $a_{j}^{s m f}$ ensures that WFS achieves a spatial matched filter. Consequently, all $\hat{E}_{j}^{s m f}$ are equivalent to their corresponding $\hat{T}_{m_{0} j}^{*}$ up to the global phase difference $\phi_{\text {ref }}$,

$$
\hat{E}^{s m f}=e^{i \phi_{r e f}}\left(\hat{\mathbf{T}}^{*}\right)^{t} F^{(0)}
$$


where $F^{(0)}$ selects the $m_{0}$-th row of the transmission matrix. Finally, a change of basis leads back to the transducer basis in the emitter plane

$$
E^{s m f}=\mathbf{H} \hat{E}^{s m f}=e^{i \phi_{r e f}} \mathbf{H}\left(\hat{\mathbf{T}}^{*}\right)^{t} F^{(0)}=N e^{i \phi_{r e f}}\left(\mathbf{T}^{*}\right)^{t} F^{(0)},
$$

showing, that the wavefront shaping algorithm fulfils the condition of the spatially matched filter (Eq. 7.5). The emission is equivalent to the one in a time reversal experiment. With the definition of the Hadamard matrix, $\mathbf{H H}^{\mathbf{t}}=\mathbf{N} \mathbf{1}$, the prefactor $\mathrm{N}$ arises since we omitted the equivalent transformation in the receiver plane. Since this proportionality factor does not impair any conclusions (see Eq. 7.5), we omit it in the following.

Theoretical enhancement factor. After optimization for focusing on point $\mathrm{m}_{0}$, the emitted signal from transducer $n$ is

$$
E_{n}^{s m f}=\frac{T_{m_{0} n}^{*}}{\sqrt{\sum_{n}\left|T_{m_{0} n}\right|^{2}}}
$$

where the denominator normalizes the transmission to ensure a given input energy of the emission. The received signal at focus point $m_{0}$ is

$$
F_{m_{0}}=\frac{\sum_{n} T_{m_{0} n} T_{m_{0} n}^{*}}{\sqrt{\sum_{n}\left|T_{m_{0} n}\right|^{2}}}=\sqrt{\sum_{n}\left|T_{m_{0} n}\right|^{2}}
$$

The average energy received at the focus point $m_{0}$ is

$$
J^{(1)}=\left\langle\left|F_{m_{0}}\right|^{2}\right\rangle=\left\langle\sum_{n}\left|T_{m_{0} n}\right|^{2}\right\rangle=N\left\langle\left|T_{m_{0} n}\right|^{2}\right\rangle,
$$

where the brackets denote the statistical average. We assume that the transmission coefficients are independent random variables and follow a circular Gaussian distribution. Without optimization, the normalized initial emission is

$$
E_{n}=\frac{1}{\sqrt{\sum_{n} 1^{2}}}=\frac{1}{\sqrt{N}}
$$

Then, the average received signal on point $m_{0}$ is

$$
J^{(0)}=\left\langle\left|F_{m_{0}}\right|^{2}\right\rangle=\left\langle\frac{\sum_{n} T_{m_{0} n} \sum_{n^{\prime}} T_{m_{0} n^{\prime}}^{*}}{(\sqrt{N})^{2}}\right\rangle=\left\langle\left|T_{m_{0} n}\right|^{2}\right\rangle .
$$

The average enhancement, which also gives the signal to background ratio to other non-optimized modes, is therefore given by

$$
\eta=\frac{J^{(1)}}{J^{(0)}}=N .
$$


Initial works in optics [13] used spatial light modulators which were limited to phaseonly control of the emitted amplitudes, such that the emission is given by

$$
E_{n}^{p o}=\frac{1}{\sqrt{N}} \frac{T_{m_{0} n}^{*}}{\left|T_{m_{0} n}\right|}
$$

The amplitude at the focus is

$$
F_{m_{0}}=\frac{1}{\sqrt{N}} \sum_{n}\left|T_{m_{0} n}\right|
$$

In this case the received energy at focus point $m$ is

$$
J^{(2)}=\left\langle\left|F_{m_{0}}\right|^{2}\right\rangle=\left\langle\left|T_{m_{0} n}\right|^{2}\right\rangle+(N-1)\left\langle\left|T_{m_{0} n}\right|\right\rangle^{2}
$$

Hence the enhancement is lowered by the well-known prefactor

$$
\frac{J^{(2)}}{J^{(1)}} \approx \frac{\left\langle\left|T_{m_{0} n}\right|\right\rangle^{2}}{\left\langle\left|T_{m_{0} n}\right|^{2}\right\rangle}=\frac{\pi}{4} \approx 0.8 .
$$

Extension from narrow-band to broadband optimization. During the first phase, we achieve spatial focusing for all frequency components contained in emission spectrum of the broadband pulse with its bandwidth $\Delta \omega_{b b}$. Since the transmission coefficients $T_{n m}(\omega)$ (Eq. 7.1) are random variables of frequency with a correlation length $\delta \omega<\Delta \omega_{b b}$, the optimal emission $E^{s m f}(\omega)$ (Eq. 7.16) has to be determined frequency-resolved for intervals $\Delta \omega<\delta \omega$.

The Hadamard algorithm is performed frequency by frequency in steps of $\Delta \omega$, each time using a narrowband pulse with a Gaussian spectrum of a bandwidth $\Delta \omega$ for the emission. For the narrowband emissions, we can assume that the linear intensity can be readily obtained by $I=\sqrt{J^{S H}}$ for the calculation of the transmission coefficients (Eq. 7.14).

When afterwards the full broadband signal with the optimal coefficients $E^{s m f}(\omega)$ is emitted, spatially matched focusing is achieved. Contrary to the time reversal experiment, temporal focusing can not yet be expected. For this, all frequency components have to be in phase, which is intrinsically fulfilled by time reversal. After the wavefront shaping optimization, an unknown phase factor $e^{i \phi_{r e f}(\omega)}$ remains which will be corrected in phase two of the algorithm.

\section{Phase two: from spatial to spatiotemporal focusing}

During the second phase, the spatially focused signal is additionally focused in the time-domain. For that, the phase factor $e^{i \phi_{r e f}(\omega)}$ needs to be determined and its conjugate equally multiplied to all emissions $E_{n}^{s m f}(\omega)$. Such spatially invariant phase filtering leads only to a temporal redistribution of the signal at the focal point. The time-integrated intensity at the focus is unaltered by the filtering process, excluding it as an appropriate feedback signal. Instead of detecting the linear intensity at the focal spot, we place a nonlinear detector which allows us to measure the time-integrated second-order response $J_{S H}$ as described by Eq. 7.7. The nonlinear signal $J_{S H}$ is sensitive for a temporal redistribution of the pulse energy. Used as a feedback signal, 
it reaches its maximum when the transmitted signal is shortest in duration, corresponding to the so-called Fourier-limit, when the waves at all frequencies are in phase at the focus. This technique has similarly been used in optics for the compression of dispersed ultrashort laser pulses [133].

The most straight-forward approach is an iterative sequential algorithm equivalent to the spatial optimization scheme described in the beginning of the section. The frequency range of the broadband emission spectrum is subdivided into intervals $\Delta \omega<\delta \omega$. Within each iteration $j$ of the optimization, we emit the full signal as determined for optimal spatial focusing, $E_{n}^{s m f}$, but modified with a spatial invariant phase mask $e^{i \Delta \phi(\omega)}$. Step by step, all frequency intervals are addressed successively. Before the first iteration, the phase mask is $\Delta \phi(\omega)=0 \forall \omega$. In step $j$, the phase mask is modified only at the frequency $\omega_{j}$. We perform several emissions with the phase $\Delta \phi\left(\omega_{j}\right)$ increasing from $\Delta \phi\left(\omega_{j}\right)=0$ to $\Delta \phi\left(\omega_{j}\right)=2 \pi$, while the second harmonic energy $J_{j}^{S H}$ is recorded.

In the following we describe the behaviour of $J_{j}^{S H}$ as a function of $\Delta \phi\left(\omega_{j}\right)$ during one step of the algorithm. For a more compact notation we drop the index $j$ and the frequency $\omega_{j}$ in this paragraph. We denote $F_{1}(t) e^{i \Delta \phi}$ the contribution wave field of the modified narrowband signal at the detector and $F_{2}(t)$ the collective wave field of all other unmodified contributions. The argument of the integral in Eq. 7.7 writes as

$$
\begin{aligned}
\left|F_{m_{0}}^{2}(t)\right|^{2}(\Delta \phi) \propto & \left|F_{1}^{2}(t)\right|^{2}+\left|F_{2}^{2}(t)\right|^{2}+4\left|F_{1}(t)\right|^{2}\left|F_{2}(t)\right|^{2}+ \\
& \Re\left\{4 F_{1}(t) F_{2}^{*}(t)\left|F_{1}(t)\right|^{2} e^{i \Delta \phi}\right\}+ \\
& \Re\left\{2 F_{1}^{2}(t)\left(F_{2}^{*}(t)\right)^{2} e^{2 i \Delta \phi}\right\}+ \\
& \Re\left\{4 F_{1}(t) F_{2}^{*}(t)\left|F_{2}(t)\right|^{2} e^{i \Delta \phi}\right\} .
\end{aligned}
$$

In the initial stage of the optimization, $F_{2}(t)$ will be largely random and spread out in time almost as much a single narrowband pulse $F_{1}(t)$. The time-integrated signal $J^{S H}$ will change as a function of phase offset $\Delta \phi$ by a both contribution proportional to $\cos (\Delta \phi)$ and a contribution proportional to the second-order term $\cos (2 \Delta \phi)$. After a few steps of the algorithms, the contribution $F_{2}(t)$ will be much larger than the contribution $F_{1}(t)$, as a number of frequencies start to interfere constructively. The change of the time-integrated signal $J^{S H}$ is then dominated by the last term in Eq. 7.27 and well-described by only the contribution proportional to $\cos (\Delta \phi)$.

For each series of emissions for the phase offset $\Delta \phi\left(\omega_{j}\right)$ applied at frequency $\omega_{j}$, $J_{j}^{S H}$ follows a cosine behaviour $\Delta J_{j}^{S H} \propto \cos \left[\Delta \phi\left(\omega_{j}\right)+\phi_{S H}(\omega)\right]$ with a phase offset $\phi_{S H}\left(\omega_{j}\right)$, which depends on the actual phases of all other frequency components. The optimum phase offset $\Delta \phi\left(\omega_{j}\right)=-\phi_{S H}\left(\omega_{j}\right)$ is found at the point where $J_{j}^{S H}$ reaches its maximum. The modified phase mask is subsequently used in the next step, in which the next frequency interval $\omega_{j+1}=\omega_{j}+\Delta \omega$ is handled in the same way. This way, the independent frequency components of the signal are set in phase at the focus step by step and the phase mask $e^{i \Delta \phi(\omega)}$ converges towards the conjugate of $e^{i \phi_{r e f}(\omega)}$. 


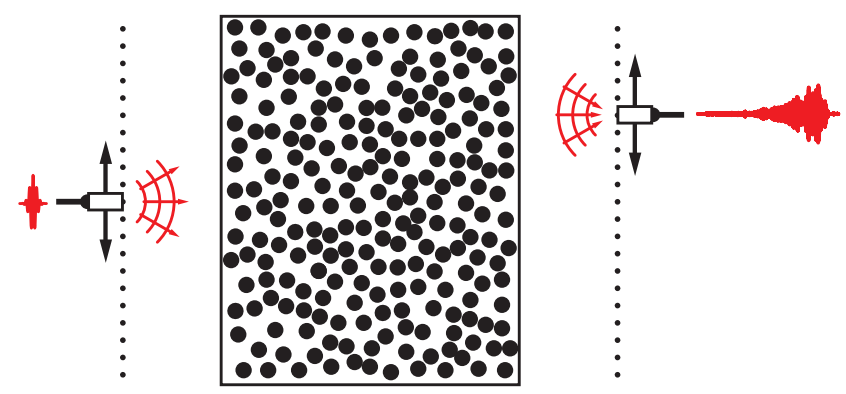

Figure 7.2: Measurement of the transmission matrix. From $N$ points in the emitter plane on the left, broadband signals are transmitted through a two-dimensional multiple scattering medium and recorded at $M$ points in the receiver plane on the right. Details of the experiment are specified in Sec. 7.3.

\subsection{Experiment and simulations}

\subsubsection{Transfer matrix measurement}

As a first step of the experiment, we recorded the transfer matrices of a multiple scattering medium over a broad range of frequencies. This way we conveniently obtain all the information about the given random medium which is needed to perform a comparative simulation of the wavefront shaping experiment and the time reversal experiment. The experimental setup consists of two identical transducers, one used as emitter and the other as receiver respectively, placed in water on opposite sides of the medium (Fig. 7.2). The latter is an arrangement (thickness $\mathrm{L}=80 \mathrm{~mm}$ ) of parallel steel rods with density $18 \mathrm{~cm}^{-2}$ and a rod diameter $0.8 \mathrm{~mm}$. Both the emitting and the receiving transducer can be translated on a line parallel to the medium, on which we defined each 128 emitter points and 128 receiver points spaced by $0.4 \mathrm{~mm}$. From each emitter point, we sent an ultrasonic pulse at a central frequency of $3.5 \mathrm{MHz}$ with a relative bandwidth of $31 \%$. In this frequency range the mean free path of the medium is $l \approx 4 \mathrm{~mm}$ as determined in [162]. The transmitted signals were recorded on all receiver points with a sampling frequency of $10 \mathrm{MHz}$. From the Fourier transform of the temporal signal, we obtain the transfer matrices in the considered frequency range (Eq. 7.1). We determined the correlation of the transmission coefficients both in the spatial and in the frequency domain by

$$
\begin{aligned}
C_{\omega}(\Delta \omega) & =\left\langle T_{n m}(\omega) T_{n m}^{*}(\omega+\Delta \omega)\right\rangle_{n, m} \\
C_{r}(\Delta r) & =\left\langle T_{n m}(\omega) T_{n(m+\Delta r)}^{*}(\omega)\right\rangle_{n, \omega}
\end{aligned}
$$

where the average is performed over the denoted matrix coefficients in Eq. 7.28 and over the first matrix entry and frequencies in Eq. 7.29. From the full width at half maximum of the correlation functions, $\delta \omega=\operatorname{FWHM}\left(\left|C_{\omega}\right|\right)=5.14 \mathrm{kHz}$ and $\operatorname{FWHM}\left(\left|C_{r}\right|\right)=1.04 \mathrm{~mm}$, we can determine that we have $1 / 5.14 \mathrm{kHz}$ independent frequencies per frequency unit and $N=(128 \cdot 0.40 \mathrm{~mm}) / 1.04 \mathrm{~mm}=49.2$ independent emitter points available [101].

With the transfer matrices at hand, we can calculate the linear response of the system for arbitrary signals emitted either from the 'emitter' or 'transducer' side. 

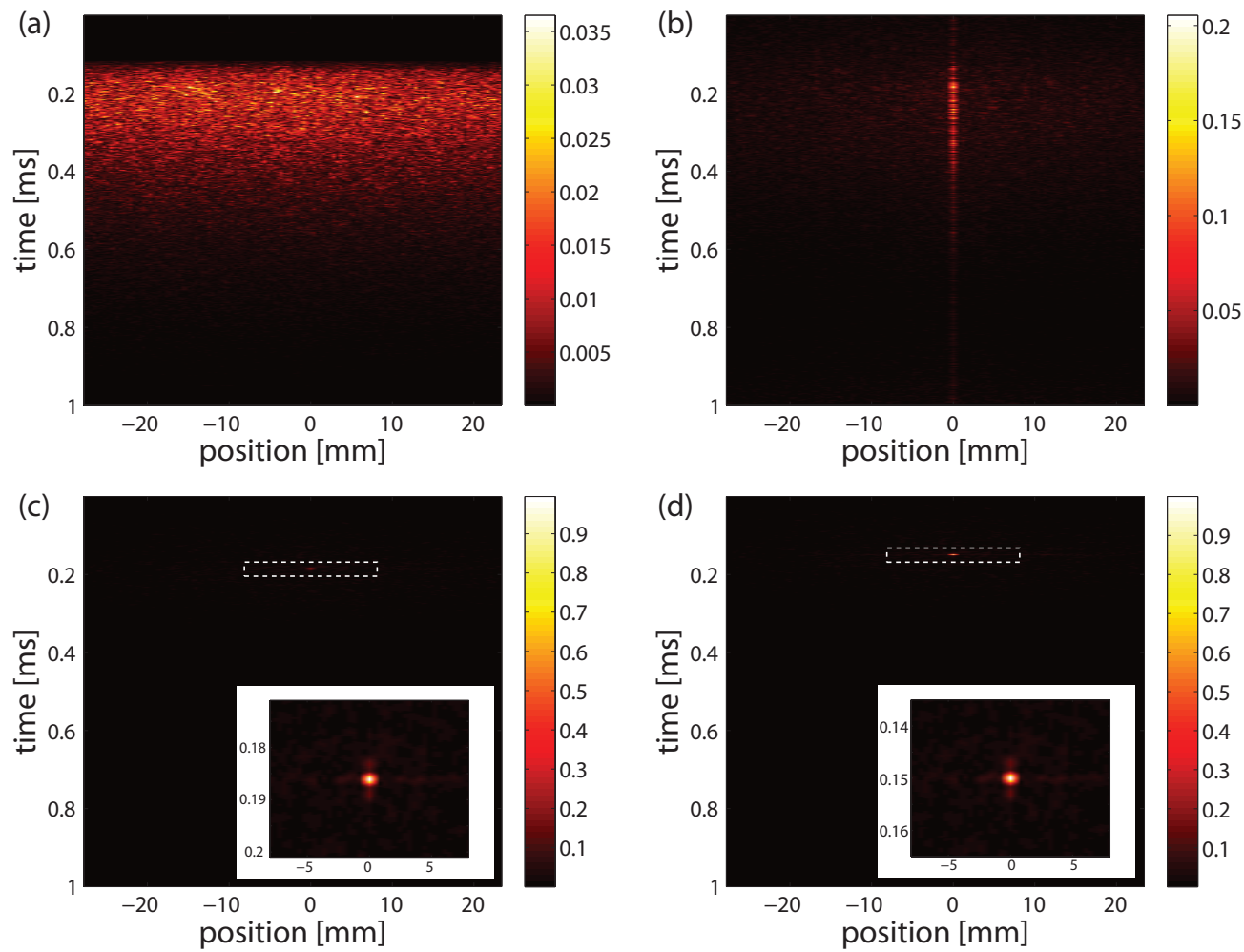

Figure 7.3: Received amplitude for non-optimized emission (a), for spatial wavefront shaping (b) and subsequent temporal focusing (c), and for comparison, for the time reversal focus (d). The areas in the dashed rectangles are plotted magnified in the inlays.

\subsubsection{Wavefront shaping simulation}

In the following we demonstrate optimal spatiotemporal focusing by a simulation of the described wavefront shaping experiment based on the measured transfer matrix and the nonlinear detector described in Eq. 7.7. It is worth to point out that alternatively to our approach here, the experiment could also be directly implemented without recording the transfer matrices.

For all following calculations, we emitted pulses with a Gaussian spectral function of $10 \%$ bandwidth around the central frequency from all transducers. A typical signal in the receiver plane is plotted in Fig. 7.3(a) and Fig. 7.4(a). The energy is spread widely both temporally and spatially. The ballistic part of the wave has disappeared, which confirms that the medium is strongly scattering.

\section{Phase one: spatial focusing}

Starting from this emission we performed a frequency-resolved wavefront shaping optimization as described in Sec. 7.2.4. The bandwidth of the narrowband pulses is chosen to $\Delta \omega=1.4 \mathrm{kHz}$ to be smaller than the correlation $\delta \omega$. In two experiments we use both emission with optimal phase and amplitude (Eq. 7.17) and the emission with optimal phase only (Eq. 7.23). The deposed energy in the receiver plane for both experiments is plotted in Fig. 7.5a. It is calculated by integrating over time the square of the wave amplitude at the receiver points (see Fig. 7.3(b) for the signal 

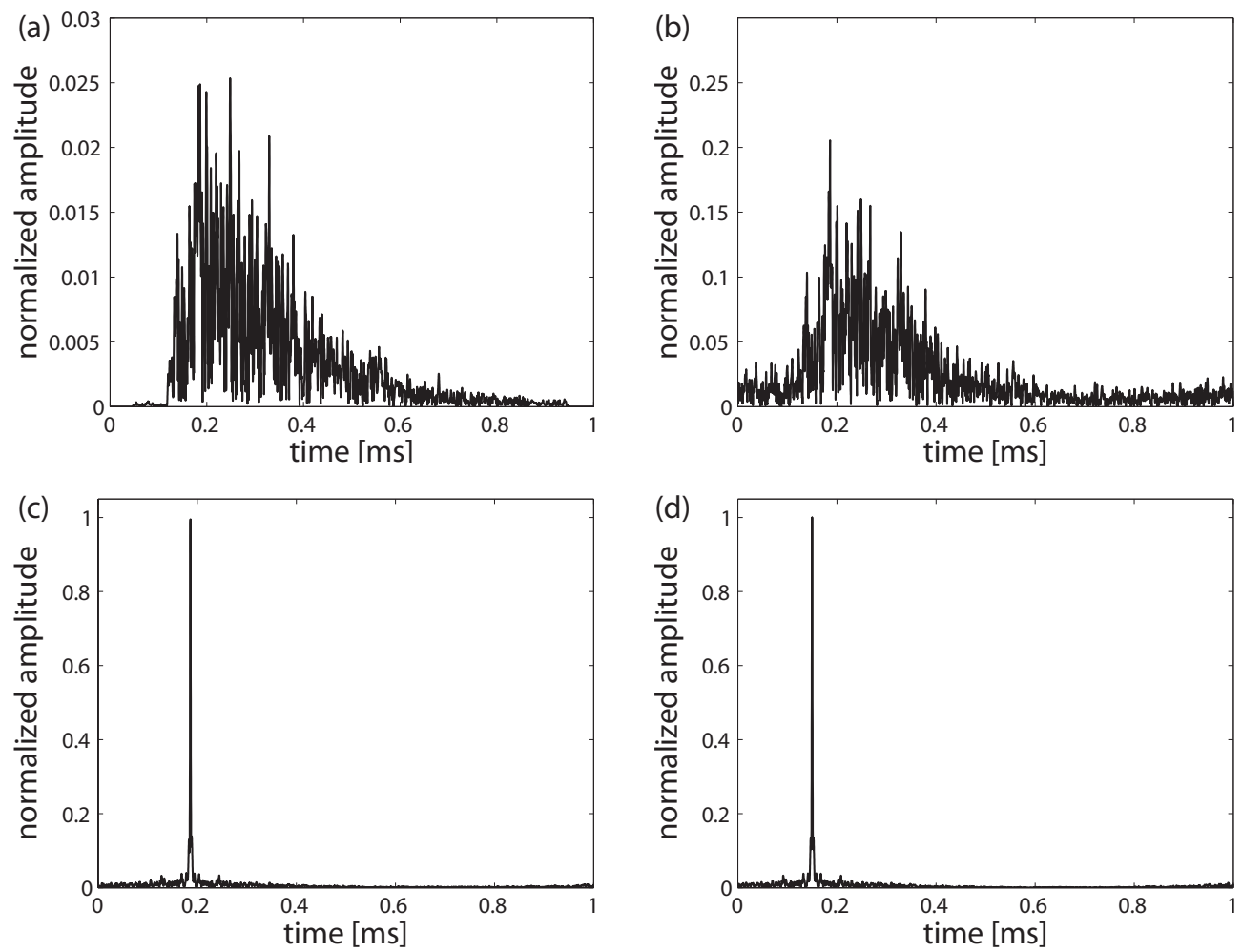

Figure 7.4: Received amplitude at the focal point for non-optimized emission (a), for spatial wavefront shaping (b) and subsequent temporal focusing (c), and for comparison, for time reversal focusing $(\mathrm{d})$.

obtained in optimizing both amplitude and phase). The optimization leads to a spatial focusing in both cases. For phase and amplitude control, the increase in energy at the focal spot is $\eta=49.2$, which corresponds perfectly to the number of estimated independent emitters $N=49.2$ (Eq. 7.22). The enhancement by the time reversal focus is slightly higher, indicating remaining deviations between the wavefront shaping emission and the time reversal emission. In further calculations, we observed that a further reduction of $\Delta \omega$ eliminates these deviations, which shows that the single-frequency TR emission and the WFS emission are identical (see Eq. 7.17 and Eq. 7.5).

For phase-only optimization, we observe an enhancement lowered by a factor 0.71 $\left(\eta_{p o}=35.1\right)$. The factor 0.8 (Eq. 7.26) is not fully reached, which we attribute to the fact, that due to the geometry of the setup not all emitters contribute equally at the focal point, effectively reducing the number of emitter points [135]. The resulting time-resolved signals for the first case are shown in Fig. 7.3(b) and Fig. 7.4(b). The energy is still spread temporally, since temporal focusing is impeded by the remaining random phase relation between independent frequency components (Eq. 7.15). In the next step, we determine and correct for this phase factor. 

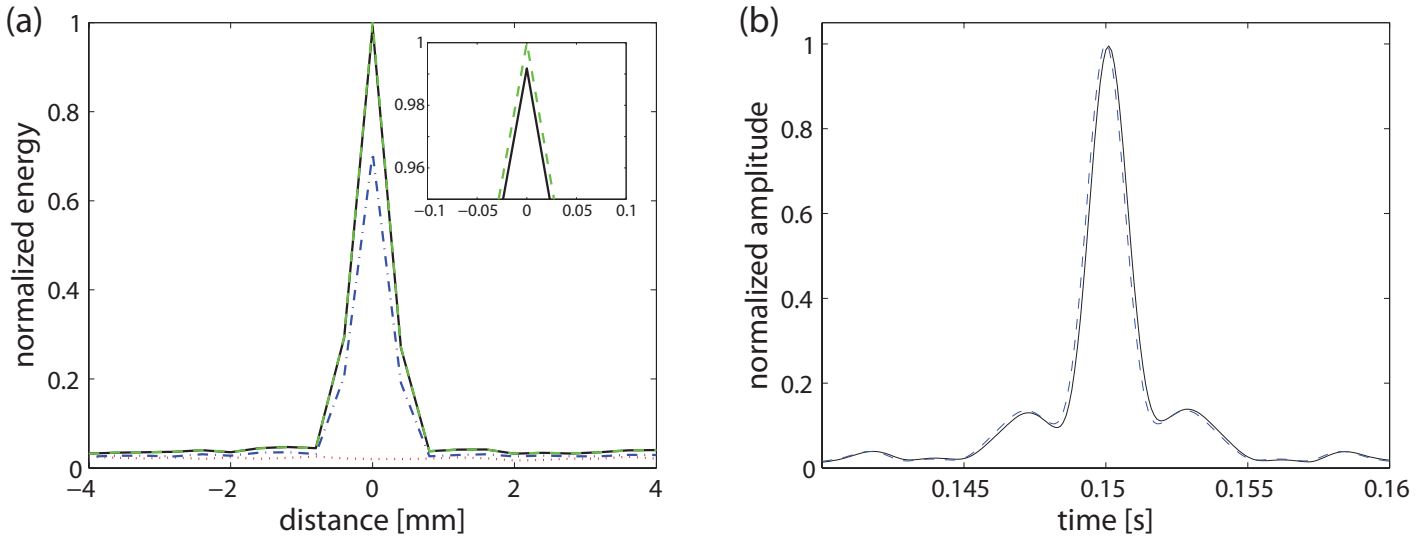

Figure 7.5: (a) Received energy before (dotted line), after wavefront shaping with phaseonly control (dash-dotted line) and combined control of amplitude and phase (solid line). The focal spot obtained with time reversal is plotted for comparison (dashed line). (b) Amplitude after wavefront shaping (solid line) and time reversal (dashed line) on the focal spot.

\section{Phase two: from spatial to spatiotemporal focusing}

Starting with the emission for spatial optimization, we perform frequency phase filtering as described in Sec. 7.2.4. After two cycles over all frequencies, the received signals are not only spatially, but also temporally focused (Fig. 7.3(c) and Fig. 7.4(c)). The resulting peak intensity can be put in context with the degrees of freedom available for the optimization. We have $N_{\text {spatial }}=49.2$ independent emitters at hand. In the frequency domain, $N_{\text {freq }}=96.3$ degrees of freedom are available calculated from the frequency correlation, $\delta \omega=5.14 \mathrm{kHz}$ and a bandwidth of $\triangle \omega_{b b}=495 \mathrm{kHz}$. Hence, the total number of degrees of freedom is $\eta_{\text {theory }}=N_{\text {spatial }} \cdot N_{\text {freq }}=4.7 \cdot 10^{3}$. We estimate the intensity enhancement to $\eta_{\exp }=6.2 \cdot 10^{3}$, comparing the peak intensity to the intensity before optimization (obtained from the transmission averaged over the transducers around the focus around the peak of the diffuse transmission, smoothed by a $60 \mu$ s window to remove temporal speckle). This number corresponds well to the order of magnitude of the number of degrees of freedom.

As a reference, we performed an equivalent time reversal experiment, which intrinsically reaches spatiotemporal focusing (Fig. 7.3(d) and Fig. 7.4(d)). The quality of the focusing from our adaptive wavefront shaping and time reversal are nearly identical (Fig. 7.5b). The small deviations are a result of small remaining phase differences which can be minimized by a reduction of the frequency steps during the spatial optimization (see above) and further iterations of the adaptive phase filtering process. As an alternative to the step-by-step algorithm we used here, so-called stimulated annealing algorithms [133] or genetic algorithms [75] should equivalently find the optimal phase relation. These algorithms are known to be robust in the presence of noise, but have the disadvantage to turn inefficient for an increasing number of degrees of freedom. 


\subsection{Conclusions}

In conclusion, we have presented a new approach for spatiotemporal focusing through complex scattering media by feedback-based wavefront shaping. In contrast to previous works, our approach is capable of reaching the limit of a spatiotemporal matched filter; the maximum possible amount of the emitted energy is deposed at the target. We showed that to achieve this, phase and amplitude of the emission need to be controlled at each frequency. In contrast to the classical time reversal experiment that achieves the ideal focus, here the direct access to the field amplitude at the target is not required as only the intensity associated to a nonlinear response needs to be detected. This point of is particular interest for many applications in wave physics where the accessible information is restricted to field intensity. For example, in optics, our method can be realized by fluorescent dye molecules as they are used for two-photon microscopy. In ultrasound therapy, it could optimize both spatial and temporal focusing of therapeutic beam through the skull bone thanks to MR radiation force imaging. Being generally applicable to all types of waves, we believe that our method is promising for a wide range of applications in imaging and sensing, and for the control of wave propagation in combination with complex media such as new metamaterials. 


\section{Summary}

This thesis deals with novel approaches to wavefront shaping methods to control wave propagation, in particular of light, in opaque scattering materials. This rapidly evolving field of research is motivated by a manifold of possible applications, for example in the development of novel imaging techniques for biomedical applications, in the performing of tests to random matrix theory, and in inventing new ways to control light in nanophotonic structures and metamaterials for communication technology, sensing and optical computing.

In the context of this work, we have developed novel experimental methods to control spatially and temporally the focusing of ultrashort laser pulses inside or through turbid media. In our optical apparatus, we shape the wavefront of the beam from the ultra-short laser by means of a phase-only spatial light modulator (SLM). At the intended focus at the backside or inside the opaque medium, each independently controlled fraction of the wavefront, typically a square segment, contributes an electric field in the form of a random speckle pulse. Setting the phase of a segment on the SLM enables us to adjust the phase of its respective contribution. At the desired focus we measure the light by an appropriate short-pulse detection scheme. We use this signal as a feedback for an iterative computer algorithm, which sets all phases so that all contributions constructively interfere at the focus and form an intense short pulse. The main challenge for these types of experiments is the implementation of a suitable feedback signal, which are outlined in the following paragraphs. A visual interpretation of our principle is that we guide the light more efficiently through paths, which lead to the focal point. At the same time we select light paths within a narrow range of lengths. Thus, the light from the selected paths reaches at the focus point at exactly the same time, recombining into an ultrashort laser pulse.

In chapter 3 we report on the first experimental demonstration of temporally controlled focusing of a short pulse through a thick layer of scattering paint. We measure the feedback by heterodyne interferometry, which provides the amplitude of the electric field at the focal point with temporal resolution. Furthermore, chapter 3 includes our theoretical predictions for the amplification of the light at the focal point and the duration of the focused short pulse, which are in very good agreement with the experimental results. 
Our work in chapters 4, 5 and 6 gradually delves into extrapolations of the method to focusing pulses not only on the backside, but also at a point inside the scattering material. For this purpose, we have devised an alternative approach to the feedback measurement. The previously used time-resolved interferometry is not applicable in this case, as it requires that the focus is accessible to an undisturbed reference pulse.

In chapter 4 we show, based on numerical simulations, that the focusing of short pulses can be achieved, circumventing the need for explicit time resolution, by making use of a non-linear feedback. A 'slow' non-linear detector produces a signal, which is the larger the more the measured light is compressed in time. Because of this relationship, the signal from the detector is sensitive to the time profile of a short pulse. We find that the amplification of the light and the duration of the formed pulse is similar to the method described in chapter 3 , but in contrast to the previous approach, this one provides no temporal control over the arrival time of the pulse.

In chapter 5 we put the idea of the slow non-linear detector into practice. We make use of nanocrystals with a high efficiency for second-harmonic generation. In our case this process corresponds to the nonlinear conversion of near-infrared light to blue light, which we quantify with a sensitive camera. On optimizing this signal, we focus short laser pulses on a single nanocrystal, which is placed at the backside of an opaque sample. As the second-harmonic generation takes place only inside the nanoparticle, we can now monitor the focusing of light by the amount of blue light produced. This can be measured from a distance, without need for time-resolved measurements. Thus, in this configuration, we can accurately measure the amount of the blue light, and compare it to our theoretical model.

In chapter 6 we show for the first time the focusing of short light pulses to the inside of an opaque medium. Now we place the nanocrystals inside the medium, rather than on the backside. The blue light generated inside the nanocrystals is scattered, similarly to the initial infrared light, in the medium. With a sensitive photodetector we measure the portion of the blue radiation that comes out of the material.

Finally, in chapter 7 we explore further possibilities of the slow nonlinear detector, however our experimental methods here differ substantially from the previous chapters. First, we are dealing with ultrasound waves in an aqueous environment instead of light waves. Second, we investigate broadband wavefront shaping: while with light we can only spatially modulate the wavefront by the SLM, regardless of the frequency of the light, with ultrasound, on top of the spatial modulation, we can easily modulate signals temporally, i.e. we can apply a different wavefront shape to each frequency component. We show that the broadband wavefront shaping in combination with a slow non-linear detector enables optimal focusing the waves for a given scattering medium. By optimal we mean that, for a given power incident onto the medium, we achieve the maximum amplitude at the focus. 


\section{Samenvatting}

Optische technieken zijn alom aanwezig in ons dagelijks leven in de vorm van displays, telecommunicatie en sensoren. In de industrie, geneeskunde en onderzoek zijn optische technieken, zoals spectroscopie en microscopie, onmisbaar om de samenstelling of de structuur van materie te onderzoeken. Een groot deel van het fundamentele onderzoek op het gebied van optische technieken gaat over het begrijpen en het beheersen van de voortplanting van licht in structuren op een schaal van nanometers met onder andere de motivatie om nieuwe generaties zonnecellen, computers of afbeeldingtechnieken te ontwikkelen.

In homogene materialen, zoals glas, kan licht met behulp van geavanceerde optische systemen met grote nauwkeurigheid worden gestuurd. Ondoorzichtige materialen, zoals biologisch weefsel, vormen echter voor vele optische toepassingen een probleem. Vanwege de wanordelijke microscopische structuur kan licht niet op een gecontroleerde manier er doorheen worden gestuurd.

In het kader van dit proefschrift hebben wij nieuwe methoden ontwikkeld om licht gecontroleerd door ondoorzichtige materialen heen te sturen. Dit onderzoeksveld werd enkele jaren geleden uitgevonden in onze groep en maakte gebruik van licht afkomstig uit continue laserbronnen. Wij hebben een methode ontwikkeld waarmee we nu ook ultrakorte lichtpulsen van gepulste lichtbronnen door en binnenin ondoorzichtige materialen kunnen sturen. Korte lichtpulsen worden voor een aantal technieken gebruikt voor het onderzoeken van kortdurende verschijnselen in de biologie, scheikunde en natuurkunde, maar deze technieken eisen meestal dat lichtverstrooiing in het materiaal een kleine rol speelt. Met onze methode is het wellicht mogelijk om deze korte lichtpulstechnieken op veel complexere systemen toe te passen, zoals biologisch weefsel. Het werk van dit proefschrift is voornamelijk gefocust op het onderzoek van elektromagnetische golven bij de frequentie van licht, maar de ontwikkelde methoden zijn ook toepasbaar op andere soorten golfen zoals microgolven of ultrasoon geluid.

\section{Korte lichtpulsen}

Onze methode maakt gebruik van een femtoseconde laser als bron voor korte lichtpulsen. De laser produceert geen continue laser bundel, maar knippert door het 
uitzenden van korte pulsen met een duur van minder dan een biljoenste seconde $\left(<10^{-12} \mathrm{~s}\right)$. Het licht van de laser in het nabij infrarood gebied heeft een trillingsperiode van ongeveer twee tot drie biljardste seconden $\left(<10^{-15} \mathrm{~s}\right)$ en de hele lichtpuls duurt typisch 10 to 100 trillingen. Het elektrische veld van een typische laserpuls is te zien in Figuur 2.2. Voor vele toepassingen van korte laserpulsen moet de lichtpuls onverstoord naar een focus worden geleidt, wat met conventionele methoden in een ondoorzichtig medium onmogelijk is.

\section{Verstrooiing van korte lichtpulsen in ondoorzichtige materialen}

Ondoorzichtige witte materialen, zoals papier, tanden of verflagen, bestaan uit een wanordelijke structuur van kleine deeltjes waar het licht op botst en van richting verandert. Dit proces wordt verstrooiing genoemd. Licht van een gerichte bundel dat in het medium verstrooid wordt raakt zijn herinnering aan zijn oorspronkelijke voortplantingsrichting kwijt en verspreidt zich (gemiddeld) gelijkmatig in alle richtingen. Laserlicht dat door een verstrooiend materiaal heen gaat, vormt een zogenaamd spikkelpatroon: het wanordelijk verstrooide licht interfereert tot een willekeurig patroon van heldere en donkere stippen. Korte laserpulsen worden gedurende de voortplanting in een verstrooiend medium ook in het tijdsdomein verstoord. De inkomende bundel wordt gesplitst en verdeelt zich over het grote aantal van de mogelijke paden door het medium. Een deel van het licht legt korte paden door het materiaal af en bereikt een punt achter het medium eerder dan pulsen die via hele lange paden lopen. In een typische verflaag van maar een paar micrometer dik kan dit verschil in aankomsttijd al vele malen groter zijn dan de oorspronkelijke duur van de laserpuls. Daarom bereikt het gepulste licht de achterkant van een verstrooiend medium niet als een onverstoord puls, maar een zogenaamde spikkelpuls, een serie van wanordelijk getimede laserpulsen verdeeld over een lang tijdsduur.

\section{Sturen van korte laserpulsen door een verstrooiend medium}

In het kader van dit onderzoek hebben wij voor het eerst een methode aangetoond om een korte lichtpuls in of achter een ondoorzichtig medium te sturen en te focusseren. In onze optische apparatuur vormen we de ruimtelijke structuur van de invallende bundel, het golffront, met behulp van een spatial light modulator (SLM), een soort tweedimensionaal LCD. Met de SLM delen we de laserstraal in vele kleine bundels waarvan we de fase van het licht apart kunnen instellen. Bij het beoogde focus draagt elke bundel een verstrooide spikkelpuls bij aan het elektrisch veld van het gemeten signaal. Door de fase van een bundel via de SLM in te stellen, kunnen we de fase van de bijhorende verstrooide spikkelpuls veranderen. Aan het gewenste focus meten we de intensiteit en pulsduur van het gearriveerde licht. Dit signaal gebruiken we als feedback voor een iteratief computeralgoritme waarmee we alle fases instellen zodat alle bundels constructief met elkaar interfereren bij de focus en een intense korte puls vormen. Een treffende interpretatie van ons experiment is dat we het licht enerzijds efficinter door paden leiden die naar het focuspunt voeren. Anderzijds wordt het licht ook met voorkeur aan paden gekoppeld met een bepaalde lengte. Daardoor komt het licht van verschillende paden op precies hetzelfde moment aan op het focuspunt, waardoor het wordt gebundeld tot een ultrakorte laserpuls. 


\section{Belangrijkste resultaten}

In hoofdstuk 3 presenteren wij de eerste experimentele demonstratie van het focusseren van een korte lichtpuls dwars door een dikke laag verstrooiende verf heen. De uitdaging voor dit experiment met korte lichtpulsen is vooral het implementeren van een geschikt feedback signaal voor het iteratieve computeralgoritme. In dit experiment meten we de feedback met behulp van een gevoelige interferometrische techniek die de tijdsopgeloste amplitude van het elektrische veld bij het focus levert. Hoofdstuk 3 bevat ook onze theoretische voorspellingen voor de versterking van het licht bij het focus en de duur van de gearriveerde korte puls, die heel goed met de experimentele resultaten overeenkomen.

Het werk in hoofdstukken 4, 5 en 6 leidt geleidelijk naar ons doel korte lichtpulsen niet alleen achter, maar ook binnenin een verstrooiend materiaal te focusseren. Voor deze doelstelling hebben wij een alternatieve aanpak voor de feedback meeting bedacht. De eerder gebruikte tijdsopgeloste interferometrische techniek is hiervoor niet van toepassing omdat we op het gewenste focus binnenin het medium geen onverstoorde referentiepuls beschikbaar kunnen gebruiken.

In hoofdstuk 4 tonen wij gebaseerd op numerieke simulaties aan dat het focusseren van korte pulsen ook met een niet-lineaire feedback signaal wordt bereikt, zonder dat daarvoor en expliciete tijdsresolutie in de meting nodig is. Zo een trage niet-lineaire detector levert een hogere intensiteit als de pulsduur van het licht korter wordt. Door deze relatie is het signaal van de detector gevoelig voor het vormen van een korte puls. We tonen aan dat de versterking van het licht en de duur van de gevormde puls verglijkbaar is met de methode in hoofdstuk 3 maar in tegenstelling daaraan levert de methode geen controle over de verblijftijd van de gefocusseerde puls in het medium.

In hoofdstuk 5 brengen we het principe van de trage niet-lineaire detector in de praktijk. We maken gebruik van nanokristallen die een hoge efficintie voor tweedeharmonische generatie vertonen. Bij dit proces worden op het nanokristal twee fotonen tot een foton van de dubbele frequentie samengevoegd, wat in ons geval een conversie van nabij-infrarood licht naar blauw licht veroorzaakt. Het blauwe licht meten we dan met een gevoelige camera. We focusseren korte lichtpulsen op enkele nanokristallen, welke aan de achterkant van een ondoorzichtig medium zijn geplaatst. In deze configuratie kunnen we de hoeveelheid van het blauwe licht nauwkeurig meten en met onze theoretische modellen van het experiment verglijken.

In hoofdstuk 6 tonen wij voor de eerste keer aan hoe korte lichtpulsen binnenin een ondoorzichtig medium te focusseren zijn. Voor dit experiment zijn de nanokristallen binnenin het medium geplaatst. Het door het nanokristal uitgezonden blauwe licht wordt in gelijke mate als het oorspronkelijke rode licht in het medium verstrooid. Een deel van het blauwe licht kunnen we buiten het materiaal met een gevoelige fotodetector meten. Op deze manier kunnen we van op een afstand het licht in het focus bij het nanokristal met een trage niet-lineaire detector meten en de korte pulsen daarop focusseren.

In hoofdstuk 7 onderzoeken we verdere mogelijkheden van de trage niet-lineaire detector, maar onze experimentele methoden verschillen substantieel van de vooraangaande hoofdstukken. Ten eerste zijn onze resultaten gebaseerd op een experiment met ultrasoon geluid in plaats van licht. Ten tweede onderzoeken wij de mogelijkheden voor het breedbandig vervormen van een golffront. In tegenstelling tot de 
golffrontvorming met licht, waar we het licht met het SLM alleen ruimtelijk kunnen moduleren, kunnen we met ultrasoon geluid rechtstreeks zowel ruimtelijk als in tijd complexe signalen meten en opwekken. We tonen aan dat de breedbandige golffrontvorming in combinatie met een trage niet-lineaire detector de golven voor een gegeven verstrooiend medium optimaal focusseert. Daarmee bedoelen we dat voor een zeker invallend vermogen op het medium, de maximale amplitude bij het focus wordt bereikt. 


\section{Zusammenfassung}

Optische Technologien sind in unserem täglichen Leben allgegenwärtig in der Form von Displays, Sensoren und Telekommunikation. In Industrie, Medizin und Forschung sind optische Techniken, wie zum Beispiel Spektroskopie oder Mikroskopie, unabdingbar zur Untersuchung der Zusammensetzung und Struktur von Materialen. Ein Großteil der optischen Grundlagenforschung richtet sich darauf, Lichtausbreitung in Materialien mit Nanostrukturen zu verstehen und kontrollieren zu können. Die Motivation für diese Forschung ist unter anderem das Ziel, neue Generationen von Solarzellen, Computern oder optischer Bildgebungstechniken zu entwickeln.

In homogenen transparenten Materialien, z.B. Glas, kann Licht mit Hilfe von perfektionierten Optiken mit großer Genauigkeit dirigiert werden. Undurchsichtige, lichtstreuende Medien, wie z.B. Wolken oder biologisches Gewebe, stellen hingegen für viele optische Anwendungen ein Problem dar. In solchen opaken Materialien breitet sich das Licht nicht geradlinig aus, sondern bewegt sich durch Vielfachstreuung in ihrer ungeordneten mikroskopischen Struktur entlang nicht vorhersehbarer Pfade. Mit konventionellen Optiken kann die Richtung der Lichtausbreitung in solchen Stoffen nicht gezielt beeinflusst werden kann.

Im Rahmen dieser Dissertation haben wir neue Methoden entwickelt, um die Lichtausbreitung in opaken Medien zu kontrollieren. Dieses Forschungsfeld wurde vor einigen Jahren in unserer Arbeitsgruppe begonnen und richtete sich zunächst auf das Kontrollieren von kontinuierlichem Laserlicht. Wir haben hier Methoden entwickelt, womit nun auch ultrakurze Laserpulse von gepulsten Lichtquellen durch und in opaken, streuenden Materialien geleitet werden können. Ultrakurze Laserpulsen werden in der Biologie, Chemie und der Physik für eine Reihe von Techniken verwendet, vor allem um schnelle ablaufende Prozesse wie chemische Reaktionen zu untersuchen. Voraussetzung für diese Anwendungen ist oft, dass Lichtstreuung in den untersuchten Proben eine untergeordnete Rolle spielt. Die von uns entwickelten Methoden können dazu beitragen, den Anwendungsbereich von Ultrakurzpulstechniken auf wesentlich komplexere Systeme auszuweiten. Diese Dissertation befasst sich vor allem mit Licht, d.h. elektromagnetischen Wellen bei der Lichtfrequenz $\left(10^{15}\right.$ Schwingungen pro Sekunde), aber unsere Methoden sind auch auf andere Wellentypen, wie Mikrowellen oder Ultraschall übertragbar. 


\section{Ultrakurze Laserpulse}

Für unsere Methode nutzen wir einen Femtosekundenlaser als Quelle für die ultrakurzen Laserpulse. Dieser Laser liefert keinen kontinuierlichen Laserstrahl, sondern blitzt 80 Millionen Mal pro Sekunde, wobei jeder der Lichtblitze weniger als eine billionstel Sekunde dauert. Das ausgesendete Nahinfrarotlicht hat eine Schwingungsdauer von zwei bis drei billiardstel Sekunden, und der gesamte Puls umfasst typischerweise 10 bis 100 Schwingungen. Das elektrische Feld eines solchen Laserpulses ist in Abbildung 2.2 dargestellt. Im Folgenden erläutern wir, wie sich ein solcher Laserpuls in stark streuenden Materialien ausbreitet.

\section{Streuung von kurzen Lichtpulsen in opaken Materialien}

Opake Materialien, wie z.B. Nebel oder Papier, sind aus einer ungeordneten Struktur von kleinen Partikeln zusammengesetzt. An diesen Teilchen wird das Licht gestreut, und verändert bei diesem Prozess seine Ausbreitungsrichtung. Nachdem das Licht eines gerichteten Laserstrahls im Material vollständig gestreut wurde, kann über die ursprüngliche Ausbreitungsrichtung keine Aussage mehr getroffen werden: das Licht breitet sich diffus, d.h. im Mittel gleichmäßig in alle Richtungen, aus. In unseren Experimenten verwenden wir meist eine deckende Schicht weißer Farbe als Modellsystem eines opaken Mediums. Kohärentes Laserlicht welches durch eine solche Farbschicht scheint erzeugt ein sogenanntes Speckle: das zufällig gestreute Licht interferiert zu einem zufälligen Muster von hellen und dunklen Punkten. Ein Laserstrahl von ultrakurzen Pulsen wird durch die Schicht neben dieser räumlichen Beeinflussung auch zeitlich stark moduliert. Beim Eintritt ins Material verteilt sich das Licht auf die große Anzahl von möglichen Streupfaden durch das Medium. Ein Teil des Lichts legt kurze Wege zurück und erreicht die Rückseite der Probe schneller als Lichtpulse die zufällig sehr lange Pfade zurücklegen. In einer typischen Schicht weißer Farbe von einigen Mikrometern Dicke kann der Unterschied in Ankunftszeit viele Male grösser sein als die ursprüngliche Pulsdauer. An der Rückseite wird dadurch nicht der ursprüngliche kurze Puls gemessen, sondern ein sogenannter Specklepuls, eine Sequenz von zeitlich über einen großen Zeitraum zufällig verteilten Pulsen. Ein Beispiel eines solchen Specklefelds ist in Abbildung 2.5 illustriert.

\section{Steuern von kurzen Lichtpulsen in streuenden Medien}

Im Rahmen dieser Arbeit haben wir erstmals eine Methode demonstriert, mit der sich kurze Lichtpulse in opaken Stoffen und hindurch steuern und fokussieren lassen. In unserer optischen Apparatur wird die räumliche Struktur des einfallenden Laserstrahls, die sogenannte Wellenfront mit einem räumlichen Lichtmodulator (Englisch: spatial light modulator, SLM) geformt. Technisch gesehen ist das SLM ein spezielles einstellbares, zweidimensionales, Flüssigkristalldisplay. Mit dem SLM unterteilen wir den Laserstrahl in viele kleine Strahlen. Bei jedem der Teilstrahlen können wir unabhängig die Phase des Lichts einstellen, indem wir das Licht mit dem SLM kontrolliert um bis zu einer Schwingungsperiode verzögern. An einem gewünschten Fokus hinter der streuenden Probe trägt jeder Teilstrahl ein kleines Lichtsignal, einen gestreuten Specklepuls zum Gesamtsignal bei. Indem wir die Phase eines Teilstrahls mit 
dem SLM einstellen, können wir die Phase des Specklepulses am gewünschten Fokus anpassen. Dort messen wir das ankommende Licht mit einer geeigneten Detektionstechnik für kurze Lichtpulse. Dieses Signal nutzen wir als Rückkopplung für einen iterativen Computeralgorithmus, und alle Phasen der Teilstrahlen so zu optimieren, das diese im Fokus konstruktiv interferieren und einen kurzen, intensiven Laserpuls formen. Eine anschauliche Interpretation diesen Prinzips ist, dass wir das Licht einerseits effizienter durch Streupfade leiten die zum Fokus führen. Gleichzeitig wählen wir mit unserer Methode auch gezielt Pfade mit einer bestimmten Länge aus. Das Licht welches sich entlang der ausgewählten Wege ausbreitet erreicht den Fokus zum gleichen Zeitpunkt und summiert sich zu einem kurzen Puls.

\section{Wichtigste Resultate}

In Kapitel 3 präsentieren wir die erste experimentelle Demonstration für das räumliche und zeitliche Steuern von ultrakurzen Laserpulsen durch ein dickes streuendes Medium. Die Herausforderung bei diesem Experiment liegt vor allem in der Implementierung eines geeigneten Feedbacksignals für den iterativen Optimierungsalgorithmus. Hier messen wir das Feedback mit Hilfe einer hochsensitiven interferometrischen Technik, welche die zeitaufgelöste Amplitude des elektrischen Feldes am gewünschten Fokus liefert. Kapitel 3 beinhaltet im Weiteren unser theoretisches Modell für die zu erzielende Verstärkung des Lichts am Fokus und die zeitliche Ausdehnung des kurzen Laserpulses. Das Modell und unsere experimentellen Ergebnisse stimmen gut überein.

Die Kapitel 4, 5 und 6 führen uns Schritt für Schritt zu unserem Ziel, Lichtpulse nicht nur durch ein Material hindurch, sondern auch im Material selbst fokussieren zu können. Zu diesem Zweck haben wir eine alternative Methode für die Feedbackmessung entwickelt. Die im vorangehenden Kapitel genutzte zeitaufösende Interferometrie ist ungeeignet, da wir am gewünschten Fokus, der sich innerhalb unserer ungeordneten Struktur befindet, keinen ungestreuten Referenzpuls verfügbar machen können. In Kapitel 4 zeigen wir auf Basis von numerischen Simulationen, dass das Fokussieren von kurzen Lichtpulsen auch mit einem nichtlinearen Detektor als Feedback erreicht wird, ohne dass dafür explizite Zeitauflösung in der Messung benötig wird. Solch ein träger nichtlinearer Detektor liefert ein größeres Signal, je kürzer die Pulsdauer des Lichts wird. Die Nutzung des nichtlinearen Signals als Rückkopplung führt daher automatisch zur Formung eines kurzen Pulses. Wir zeigen, dass die Verstärkung des Lichts im Fokus und die erzielte Pulsdauer mit der im vorigen Kapitel untersuchten Methode vergleichbar sind. Im Gegensatz zur interferometrischen Methode gibt der nichtlineare Detektor jedoch keine Kontrolle über die Verweildauer der fokussierten Pulse im Material.

In Kapitel 5 setzen wir das Prinzip des trägen nichtlinearen Detektors in die Praxis um. Wir verwenden Nanokristalle die eine hohe Effizienz für die Frequenzverdopplung von Licht aufweisen. Bei diesem Prozess werden im Nanokristall zwei Photonen zu einem Photon der doppelten Frequenz verschmolzen. In unserem Fall entspricht dieser Prozess der Konversion von Nahinfrarotlicht zu blauem Licht. Letzteres messen wir mit einer hochsensitiven Kamera. In unserem Experiment zeigen wir das Fokussieren von Lichtpulsen auf einzelne Nanokristallen, welche an der Rückseite eines stark streuenden Materials platziert sind. In dieser Konfiguration können wir das blaue Licht quantitativ mit hoher Genauigkeit messen und mit unseren theore- 
tischen Modellen vergleichen. In Kapitel 6 zeigen wir dann erstmals das Fokussieren von kurzen Laserpulses im inneren eines opaken Mediums. Für dieses Experiment sind die Nanokristalle im Medium eingebettet. Das durch die Nanokristalle ausgesendete blaue Licht wird vergleichbar mit dem ursprünglichen roten Licht im Material gestreut. Einen Teil davon kann von außerhalb des Mediums mit einem sensitiven Fotodetektor gemessen werden. Da die Frequenzverdopplung nur beim Nanokristall stattfindet, können wir das Fokussieren der Pulse von außerhalb des Mediums anhand der Menge des produzierten blauen Lichts quantifizieren und verfolgen, ohne dass ein zeitauflösender Detektor benötigt wird.

In Kapitel 7 erweitern wir das Konzept des räumlichen und zeitlichen Steuerns von gestreuten Wellen unter Verwendung des trägen nichtlinearen Detektors. Die verwendeten experimentellen Methoden unterscheiden sich hierbei wesentlich von den vorangehenden Kapiteln. Erstens, in diesem Kapitel führen wir die Experimente nicht mit Lichtwellen, sondern mit Schallwellen durch. Zweitens untersuchen wir neue Ansätze für das breitbandige Formen einer Wellenfront. Bei der Wellenfrontformung mit Licht wird der Lichtstrahl mit dem SLM lediglich räumlich moduliert, d.h. die Modulation ist weitgehend unabhängig von der Lichtfrequenz. Mit parallel angeordneten Ultraschallwandlern lassen sich Signale neben der räumlichen Modulation auch zeitlich modulieren, i.e., für jede Frequenz kann eine separate Wellenfront verwendet werden. Wir zeigen, dass breitbandige Wellenfrontformung in Kombination mit einem trägen nichtlinearen Detektor optimales Fokussieren erlaubt. Damit meinen wir hier, dass für eine gegebene auf das Medium eingestrahlte Leistung die maximale Pulsamplitude am Fokus erzeugt wird. 


\section{Acknowledgements}

My doctoral work would have not been possible without the invaluable help of a number of people. First of all, I would like to thank Ad Lagendijk and Mathias Fink for realizing this project and for giving me the wonderful opportunity of working in two outstanding laboratories located in two beautiful cities: the AMOLF institute in Amsterdam and the Institut Langevin in Paris.

I would like to express my deepest gratitude to my $\mathrm{PhD}$ supervisor Ad Lagendijk at AMOLF. Thank you for your trust in me all along the way. I received many good pieces of advice and encouragement from you, from which I profited in my development as a researcher, and certainly much beyond that.

I would like to thank Arnaud Tourin for his support and for the supervision of my project at ESPCI. I greatly appreciated your critical examination of all the chapters of this thesis and your helpful comments. I am very grateful that you accepted to be the copromotor for my $\mathrm{PhD}$.

I am also deeply grateful Allard Mosk from the COPS group in Twente for our fruitful collaboration on all the wavefront shaping projects with light and for sharing his vast physics expertise. Bergin Gjonaj was my comrade $\mathrm{PhD}$ in the optics lab at AMOLF. Dear Bergin, thank you most of all for your creativity, which you brought into our countless helpful discussions. Their intensity sometimes topped the one of our table tennis matches in the old building. I am deeply indebted also to Patrick Johnson for his help in writing my manuscripts and for always having an open ear and time to discuss problems coming up in my projects. I greatly appreciate your calm way of dealing with challenges.

During my doctoral work, I profited also from the help of a number of people who contributed to my work on individual projects. Many thanks to Dries van Oosten for getting me started on the heterodyne interferometry and Kobus Kuipers for proving the equipment (chapter 3). Thanks also to the staff of the AMOLF electronics workshop, and in particular Idsart Attema, for fixing the frequency mixer and to Timmo van der Beek for fabricating the scattering samples. Thank you to Mohamed Tachikirt for his assistance in the second harmonic project (chapters 5 and 6 ) and his patient help on our long road to fabricate suitable scattering samples. Credit goes to Alice Bretagne for providing the data of the ultrasound transmission 
matrix measurements and for her contribution to our manuscript (chapter 7). I also would like to thank Mathias Fink and Mickaël Tanter, who co-supervised this project and made inspiring and instructive contributions to our discussions. Further, I would like to acknowledge Jean-Luc Gennisson and Olivier Couture from Institute Langevin for their collaboration on the shear wave project and the bubble project, which unfortunately did not lead to publishable results.

I thank all members of the Photon Scattering group at AMOLF for and their critical and helpful input to my research. Besides that, thank you Bergin, Iwert, Mohamed, Paolo, Patrick, Ramy, Ronald and Sanli for making me part of a memorable team. With joy, I think back to many heated discussions, where strong opinions clashed over some tasty sandwiches on the Phoscat lunch table.

At AMOLF if first started out in the Photonic Bandgaps Group. I thank Willem Vos for giving me the opportunity to work in his group in the first place and for his supervision on the plasmonics project. Many thanks for your support and your continued curiosity about my research after our 'restart' and my transition to the wavefront shaping project.

With the COPS group from Twente I had a rich regular exchange of ideas on wavefront shaping and scattering topics. Alex, Alexander, Amandev, Bart, Marlon, Merel, Georgios, Sergej, Simon, Erwin, Danang, Elbert, Emre, Rajesh, Duygu, Bas, Jin, Femi, Elahe, Hasan, Vanessa, Henri, Jacopo, Nilda, Thomas, Pepijn, Cock, Hannie, Frerik. Thank you all for the inspiring meetings and the welcoming atmosphere of my visits in Twente. I particularly thank Georgios Ctistis for our good collaboration on the plasmonics project.

My project was funded by NWO and Shell; I would like to thank Xander Campman from Shell for his continued interest in our research at our regular meetings.

Doing a step back in time, I would like to thank the supervisor of my Diploma thesis, Tobias Brixner, for opening my door to optics research. I also thank my coworkers at this time, Daniel Wolpert and Marco Schade, who taught me many of the lab skills from which I greatly profited when doing the first experiments of my $\mathrm{PhD}$ project.

The colloquium and the poster session of the Center for Nanophotonics made an excellent preparation for conference visits. I would like to thank Albert Polman, Jaime Gomez-Rivas, Kobus Kuipers, Ewold Verhagen, Femius Koenderink and Ad Lagendijk for organizing this useful institution and for sharing their individual approaches to research with us. Thanks to all the Nanophotonics members for many instructive discussions and for the 'gezelligheid' at the lunch and the coffee table.

Een grote dank gaat aan mijn kamergenoten op AMOLF, Timmo, Claire, Maarten en Hinke voor de gezelligheid en de leuke gesprekken over voetbal en andere belangrijke dingen naast de wetenschap. The nanosoil team evolving from the AMOLF invention contest was more than just some distraction from my research. Marjon, Ivana, Nils and Timmo, it was a pleasure working together with you.

I would like to thank the members of the permanent staff at AMOLF for making the work at the institute a great experience. Thank you all for your readiness in helping, your efficiency and your enthusiasm to create a pleasant atmosphere. In particular, I thank Anouk Nelisen, Wouter Harmsen, Wiebe de Boer, Jan van Elst, Willem de Jong, Duncan Verheijde, Hinko Schoenmaker, Andre Reinders, Tatjana 
van der Ben-Cambach and Arnelli Scharbaii for technical and administrative support during the last years. A great help is that AMOLF directly provides housing to students. I thank Juanita Nahar, Lenny Heeren and Bart van Leijen for organizing and maintaining the house on Middenweg. From ESPCI, I thank Patricia Daenens, Christelle Jacquet, Stèphanie Andrès and Corinne Parescandolo for their friendly support in tackling problems such as French keyboards or administrative hurdles.

For the times outside work hours, many people contributed to making my stay in Amsterdam and Paris an unforgettable period of my life. I am grateful to Seb, Salma, Carla, Charles, Sarah, Remi and Romano. Thank you for welcoming me in your circle of friends in Paris and for having me at many pots and soirées and the weekend at the coast. Geoffroy, Alexandre, thank you for enjoyable evenings in San Jose and Cargèse over one or the other drink. Thanks to the 'La Créte' group Wojchiek, Thomas and Wei-Ning for our pleasant lunches in the heart of Paris. Aurélie and Ben, thanks for the time in 'la colocation du bonheur'.

Bergin, Ivana and Marko, thanks for that remarkable trip to Kopenhagen. Martin, thank you for being my fellow nederduitser and kitesurf buddy. Aron, Andrej, Anouk, Chris, Clara, Ernst-Jan, Felipe, Gianluca, Helen, Jean, Jeanette, Jose, Lutz, Nuria, Maga, Oleg, Paolo, Pierre, Rea, Ronald, Ruben, Sophie, Stephanie and Thomas, thank you all for the great times, let it be rabbit hunts, fuzzy nights out, parties, dinners or bicycle trips around Amsterdam. Stephan, thanks for your humorous and relaxed way of handling things, it was my pleasure sharing the Middenweg apartment.

Lutz and Simon, thank you very much for agreeing to be my 'Paranimfen' and for helping me handling the last steps of this thesis and preparing my defense.

To my oldest companions, Andreas, Christian, Daniel, Paul, Philipp and Tim, thank you for all your support over the all those years since we know each other. I am most thankful to my family for their support throughout my life, to my sisters Barbara and Susanne, my parents Irmtraud and Bernd, and my grandfather Friedel. My last and biggest thank you goes to Giulia, for her support, her love and for bearing with me in these last stressful months. Thank you for all your efforts bridging the gaps between Oxford, Paris, Freiburg and Amsterdam. Our next steps will bring us closer together. 



\section{Bibliography}

[1] G. P. Agrawal, Fiber-Optic Communication Systems, John Wiley \& Sons (2010). - p.11.

[2] T. Ito and S. Okazaki, "Pushing the limits of lithography", Nature 406, 1027 (2000). - p.11.

[3] F. Helmehen and W. Denk, "Deep tissue two-photon microscopy", Nat. Methods 2, 932 (2005). - p.11, 70, and 90.

[4] A. Villringer and B. Chance, "Non-invasive optical spectroscopy and imaging of human brain function", Trends Neurosci. 20, 435 (1997). — p.11.

[5] L. Novotny and B. Hecht, Principles of nano-optics, Cambridge University Press, Cambridge (2012). — p.11.

[6] H. A. Atwater and A. Polman, "Plasmonics for improved photovoltaic devices", Nature Mater. 9, 865 (2010). — p.11.

[7] J. L. O'Brien, "Optical quantum computing", Science 318, 1567 (2007). - p.11.

[8] J. B. Pendry, "Negative refraction makes a perfect lens", Phys. Rev. Lett. 85, 3966 (2000). - p.11.

[9] A. Ishimaru, Wave Propagation and Scattering in Random Media, Academic Press (1978). - p.11 and 23.

[10] M. C. W. van Rossum and T. M. Nieunenhuizen, "Multiple scattering of classical waves: microscopy, mesoscopy, and diffusion", Rev. Mod. Phys. 71, 313 (1999). - p.11, 24, 25, 26, 30, and 96.

[11] P. YeH and C. Gu, Optics of Liquid Crystal Displays, John Wiley \& Sons (2010). - p.12, 19, and 20.

[12] C. Maurer, A. Jesacher, S. Bernet, and M. Ritsch-Marte, "What spatial light modulators can do for optical microscopy", Laser Photon. Rev. 5, 81 (2011). - p.12.

[13] I. M. Vellekoop and A. P. Mosk, "Focusing coherent light through opaque strongly scattering media", Opt. Lett. 32, 2309 (2007). — p.12, 38, 40, 50, 69, 90, 110, and 116.

[14] I. M. Vellekoop, E. G. van Putten, A. LagendiJk, and A. P. Mosk, "Demixing light paths inside disordered metamaterials", Opt. Express 16, 67 
(2008). - p.12, 15, 38, 70, 90, 101, and 110.

[15] C. W. J. Beenakker, "Random-matrix theory of quantum transport", Rev. Mod. Phys. 69, 731 (1997). — p.12 and 27.

[16] I. M. Vellekoop and A. P. Mosk, "Universal optimal transmission of light through disordered materials", Phys. Rev. Lett. 101, 120601 (2008). - p.12.

[17] A. P. Mosk, A. LagendiJk, G. Lerosey, and M. Fink, "Controlling waves in space and time for imaging and focusing in complex media", Nat. Photon. 6, 283 (2012). - p.12, 14, and 90.

[18] S. M. Popoff, G. Lerosey, R. Carminati, M. Fink, A. C. Boccara, and S. Gigan, "Measuring the transmission matrix in optics: An approach to the study and control of light propagation in disordered media", Phys. Rev. Lett. 104, 100601 (2010). — p.12, 43, and 90.

[19] S. M. Popoff, Contrôle spatio-temporel de la lumière en milieux complexes, Ph.D. thesis, Université Paris-Diderot - Paris VII (2011). — p.12.

[20] Y. Choi, T. D. Yang, C. Fang-Yen, P. Kang, K. J. Lee, R. R. Dasari, M. S. FELD, and W. ChOI, "Overcoming the diffraction limit using multiple light scattering in a highly disordered medium", Phys. Rev. Lett. 107, 023902 (2011). - p.12 and 90.

[21] M. Cui, "A high speed wavefront determination method based on spatial frequency modulations for focusing light through random scattering media", Opt. Express 19, 2989 (2011). — p.12.

[22] D. B. Conkey, A. M. Caravaca-Aguirre, and R. Piestun, "High-speed scattering medium characterization with application to focusing light through turbid media", Opt. Express 20, 1733 (2012). — p.12.

[23] C. Stockbridge, Y. Lu, J. Moore, S. Hoffman, R. Paxman, K. TouSSAINT, and T. BIFAnO, "Focusing through dynamic scattering media", Opt. Express 20, 15086 (2012). - p.12.

[24] E. G. van Putten, D. Akbulut, J. Bertolotti, W. L. Vos, A. LAGENDIJK, and A. P. Mosk, "Scattering lens resolves sub-100 nm structures with visible light", Phys. Rev. Lett. 106, 193905 (2011). — p.12, 70, and 90.

[25] E. G. VAn PutTen, Disorder-Enhanced Imaging with Spatially Controlled Light, Ph.D. thesis, University of Twente (2011). - p.12.

[26] M. Cui and C. YANG, "Implementation of a digital optical phase conjugation system and its application to study the robustness of turbidity suppression by phase conjugation", Opt. Express 18, 3444 (2010). — p.12, 70, and 90.

[27] C.-L. Hsieh, Y. Pu, R. Grange, and D. Psaltis, "Digital phase conjugation of second harmonic radiation emitted by nanoparticles in turbid media", Opt. Express 18, 12283 (2010). — p.12, 38, 43, 70, and 90.

[28] R. Di Leonardo and S. Bianchi, "Hologram transmission through multimode optical fibers", Opt. Express 19, 247 (2010). — p.13.

[29] Y. Choi, C. Yoon, M. Kim, T. D. Yang, C. Fang-Yen, R. R. Dasari, K. J. LEE, and W. CHOI, "Scanner-free and wide-field endoscopic imaging by using a single multimode optical fiber", Phys. Rev. Lett. 109, 203901 (2012). - p.13.

[30] I. N. Papadopoulos, S. Farahi, C. Moser, and D. Psaltis, "Focusing and scanning light through a multimode optical fiber using digital phase con- 
jugation", Opt. Express 20, 10583 (2012). — p.13.

[31] B. Guonaj, J. Aulbach, P. M. Johnson, A. P. Mosk, L. Kuipers, and A. LagendiJK, "Active spatial control of plasmonic fields", Nat. Photon. 5, 360 (2011). — p.13 and 110.

[32] B. Guonaj, J. Aulbach, P. M. Johnson, A. P. Mosk, L. Kuipers, and A. LAGEndiJK, "Optical control of plasmonic bloch modes on periodic nanostructures", Nano Lett. 12, 546 (2012). — p.13.

[33] B. Guonaj, J. Aulbach, P. M. Johnson, A. P. Mosk, L. Kuipers, and A. LAGEndiJK, "Focusing and scanning microscopy with propagating surface plasmons", Phys. Rev. Lett. 110, 266804 (2013). — p.13.

[34] J. Jang, J. Lim, H. Yu, H. Choi, J. Ha, J.-H. PARK, W.-Y. OH, W. Jang, S. LEE, and Y. PARK, "Complex wavefront shaping for optimal depth-selective focusing in optical coherence tomography", Opt. Express 21, 2890 (2013). p.13 and 90 .

[35] X. Xu, H. LiU, and L. V. WANG, "Time-reversed ultrasonically encoded optical focusing into scattering media", Nat. Photon. 5, 154 (2011). — p.13.

[36] B. Judkewitz, Y. M. Wang, R. Horstmeyer, A. Mathy, and C. Yang, "Speckle-scale focusing in the diffusive regime with time reversal of varianceencoded light (TROVE)", Nat. Photon. 7, 300 (2013). — p.13 and 90.

[37] J. Bertolotti, E. G. van Putten, C. Blum, A. Lagendiuk, W. L. Vos, and A. P. Mosk, "Non-invasive imaging through opaque scattering layers", Nature 491, 232 (2012). — p.13.

[38] M. Fink, "Time reversal of ultrasonic fields. i. basic principles", IEEE Ultr. Ferro. Freq. Cont. 39, 555 (1992). — p.13.

[39] M. Fink, "Time reversed acoustics", Phys. Today 50, 34 (1997). — p.14, 34, $38,70,90$, and 110 .

[40] A. Derode, P. Roux, and M. Fink, "Robust acoustic time reversal with high-order multiple scattering", Phys. Rev. Lett. 75, 4206 (1995). — p.14 and 38 .

[41] G. Lerosey, J. de Rosny, A. Tourin, A. Derode, G. Montaldo, and M. Fink, "Time reversal of electromagnetic waves", Phys. Rev. Lett. 92, 193904 (2004). - p.14.

[42] G. Lerosey, J. de Rosny, A. Tourin, and M. Fink, "Focusing beyond the diffraction limit with far-field time reversal", Science 315, 1120 (2007). - p.14, 38, 70, 90, and 110.

[43] L. Borcea, G. Papanicolaou, C. Tsogka, and J. Berryman, "Imaging and time reversal in random media", Inverse Probl. 18, 1247 (2002). - p.14.

[44] C. S. Larmat, R. A. Guyer, and P. A. Johnson, "Time-reversal methods in geophysics", Phys. Today 63, 31 (2010). — p.14.

[45] J.-L. Thomas, F. Wu, and M. Fink, "Time reversal focusing applied to lithotripsy", Ultrasonic Imaging 18, 106 (1996). — p.14.

[46] A. Derode, A. Tourin, J. de Rosny, M. Tanter, S. Yon, and M. Fink, "Taking advantage of multiple scattering to communicate with time-reversal antennas", Phys. Rev. Lett. 90, 014301 (2003). — p.14 and 38.

[47] G. Lerosey, J. de Rosny, A. Tourin, A. Derode, G. Montaldo, and M. Fink, "Time reversal of electromagnetic waves and telecommunication", 
Radio Sci. 40 (2005). - p.14.

[48] S. Mukamel, Principles of nonlinear optical spectroscopy, Oxford University Press (1995). - p.14.

[49] W. Denk, J. H. Strickler, and W. W. WebB, "Two-photon laser scanning fluorescence microscopy", Science 248, 73 (1990). - p.14.

[50] H. Rabitz, R. de Vivie-Riedle, M. Motzkus, and K. Kompa, "Whither the future of controlling quantum phenomena?", Science 288, 824 (2000). p.14, 21, and 37 .

[51] M. Aeschlimann, M. Bauer, D. Bayer, T. Brixner, F. De Abajo, W. Pfeiffer, M. Rohmer, C. Spindler, and F. Steeb, "Adaptive subwavelength control of nano-optical fields", Nature 446, 301 (2007). — p.14 and 37.

[52] M. I. Stockman, "Ultrafast nanoplasmonics under coherent control", New J. Phys. 10, 025031 (2008). — p.14.

[53] A. Vogel, J. Noack, G. Hüttman, and G. Paltauf, "Mechanisms of femtosecond laser nanosurgery of cells and tissues", Appl. Phys. B 81, 1015 (2005). - p.14 and 37.

[54] W. Srituravanich, N. Fang, C. Sun, Q. Luo, and X. Zhang, "Plasmonic nanolithography", Nano Letters 4, 1085 (2004). - p.14.

[55] P. M. Johnson, A. F. Koenderink, and W. L. Vos, "Ultrafast switching of photonic density of states in photonic crystals", Phys. Rev. B 66, 081102 (2002). - p.14.

[56] P. Lodahl, A. F. van Driel, I. S. Nikolaev, A. Irman, K. Overgaag, D. VanmaekelberGh, and W. L. Vos, "Controlling the dynamics of spontaneous emission from quantum dots by photonic crystals", Nature 430, 654 (2004). - p.14.

[57] F. Lemoult, N. Kaina, M. Fink, and G. Lerosey, "Wave propagation control at the deep subwavelength scale in metamaterials", Nat. Phys. 9, 55 (2013). - p.14.

[58] I. M. Vellekoop, A. LagendiJK, and A. P. Mosk, "Exploiting disorder for perfect focusing", Nat. Photon. 4, 320 (2010). — p.14.

[59] A. M. WeInER, "Femtosecond pulse shaping using spatial light modulators", Rev. Sci. Instrum. 71, 1929 (2000). — p.14, 21, 42, and 110.

[60] P. Hariharan, Optical Interferometry, Academic Press (2003). — p.14.

[61] I. M. Vellekoop, Controlling the propagation of light in disordered scattering media, Ph.D. thesis, University of Twente (2008). — p.17.

[62] M. Wollenhaupt, A. Assion, and T. Baumert, "Femtosecond laser pulses: Linear properties, manipulation, generation and measurement", in "Springer Handbook of Lasers and Optics", Springer New York, pp. 937-983 (2007). — p.18, 19, and 32 .

[63] J.-C. Diels and W. Rudolph, Ultrashort Laser Pulse Phenomenon: Fundamentals, Techniques, and Applications on a Femtosecond Time Scale, Elsevier/Academic Press (2006). - p.18, 32, and 34.

[64] C. Rulliere, Femtosecond Laser Pulses: Principles and Experiments, Springer (2005). - p.18 and 32.

[65] A. Guinier, X-ray Diffraction in Crystals, Imperfect Crystals, and Amorphous 
Bodies, Courier Dover Publications (1994). - p.19.

[66] E. G. van Putten, I. M. Vellekoop, and A. P. Mosk, "Spatial amplitude and phase modulation using commercial twisted nematic LCDs", Appl. Optics 47, 2076 (2008). - p.20.

[67] V. Nikolenko, B. O. Watson, R. Araya, A. Woodruff, D. S. PeTERKA, and R. YUSTE, "SLM microscopy: Scanless two-photon imaging and photostimulation with spatial light modulators", Front. Neural Circuits 2 (2008). - p.21.

[68] P. Kner, B. B. Chhun, E. R. Griffis, L. Winoto, and M. G. L. Gustafsson, "Super-resolution video microscopy of live cells by structured illumination", Nat. Methods 6, 339 (2009). — p.21.

[69] J. Durnin, J. J. Miceli, and J. H. Eberly, "Diffraction-free beams", Phys. Rev. Lett. 58, 1499 (1987). - p.21.

[70] F. O. Fahrbach, P. Simon, and A. Rohrbach, "Microscopy with selfreconstructing beams", Nat. Photon. 4, 780 (2010). — p.21.

[71] P. Polynkin, M. Kolesik, J. V. Moloney, G. A. Siviloglou, and D. N. Christodoulides, "Curved plasma channel generation using ultraintense airy beams", Science 324, 229 (2009). — p.21.

[72] T. Brixner and G. Gerber, "Femtosecond polarization pulse shaping", Opt. Lett. 26, 557 (2001). - p.21.

[73] P. Tournois, "Acousto-optic programmable dispersive filter for adaptive compensation of group delay time dispersion in laser systems", Opt. Commum. 140, 245 (1997). - p.21.

[74] A. Assion, T. Baumert, M. Bergt, T. Brixner, B. Kiefer, V. Seyfried, M. Strehle, and G. Gerber, "Control of chemical reactions by feedback-optimized phase-shaped femtosecond laser pulses", Science $\mathbf{2 8 2}$, 919 (1998). — p.21.

[75] T. Brixner, M. Strehle, and G. Gerber, "Feedback-controlled optimization of amplified femtosecond laser pulses", Appl. Phys. B 68, 281 (1999). - p.21 and 121.

[76] J. Herek, W. Wohlleben, R. Cogdell, D. Zeidler, and M. Motzkus, "Quantum control of energy flow in light harvesting", Nature 417, 533 (2002). — p.21 and 37 .

[77] M. M. Wefers, K. A. Nelson, and A. M. Weiner, "Multidimensional shaping of ultrafast optical waveforms", Opt. Lett. 21, 746 (1996). — p.22.

[78] R. Tyson, Principles of Adaptive Optics, CRC Press (2010). — p.22.

[79] H. C. Hulst and H. C. V. D. Hulst, Light scattering by small particles, Courier Dover Publications (1957). - p.23.

[80] E. Akkermans, P. E. Wolf, and R. Maynard, "Coherent backscattering of light by disordered media: Analysis of the peak line shape", Phys. Rev. Lett. 56, 1471 (1986). - p.25.

[81] A. LagendiJk, R. VReeker, and P. De VRIEs, "Influence of internal reflection on diffusive transport in strongly scattering media", Phys. Lett. A 136, 81 (1989). - p.25.

[82] J. X. Zhu, D. J. Pine, and D. A. Weitz, "Internal reflection of diffusive light in random media", Phys. Rev. A 44, 3948 (1991). — p.25. 
[83] M. U. VERA and D. J. Durian, "Angular distribution of diffusely transmitted light", Phys. Rev. E 53, 3215 (1996). - p.25.

[84] C. F. Bohren and D. R. Huffman, Absorption and scattering of light by small particles, Wiley (1983). - p.25 and 96.

[85] J. F. DE BoER, Optical fluctuations on the tranmsission and reflection of mesoscopic systems, Ph.D. thesis, University of Amsterdam (1995). — p.25 and 26.

[86] D. J. Thouless, "Maximum metallic resistance in thin wires", Phys. Rev. Lett. 39, 1167 (1977). - p.26.

[87] J. W. Goodman, Speckle Phenomena in Optics: Theory and Applications, Roberts \& Company Publ. (2010). — p.27.

[88] R. Berkovits and S. Feng, "Correlations in coherent multiple scattering", Phys. Rep. 238, 135 (1994). - p.29.

[89] E. Akkermans, Mesoscopic Physics of Electrons and Photons, Cambridge University Press (2007). - p.29 and 30.

[90] S. Feng, C. Kane, P. A. Lee, and A. D. Stone, "Correlations and fluctuations of coherent wave transmission through disordered media", Phys. Rev. Lett. 61, 834 (1988). - p.29 and 89.

[91] I. Freund, M. Rosenbluh, and S. Feng, "Memory effects in propagation of optical waves through disordered media", Phys. Rev. Lett. 61, 2328 (1988). - p.29.

[92] R. Berkovits, M. Kaveh, and S. Feng, "Memory effect of waves in disordered systems: A real-space approach", Phys. Rev. B 40, 737 (1989). — p.30.

[93] B. Shapiro, "Large intensity fluctuations for wave propagation in random media", Phys. Rev. Lett. 57, 2168 (1986). — p.30.

[94] M. C. W. van Rossum, T. M. Nieuwenhuizen, and R. Vlaming, "Optical conductance fluctuations: Diagrammatic analysis in the landauer approach and nonuniversal effects", Phys. Rev. E 51, 6158 (1995). — p.30.

[95] B. A. van Tiggelen, P. Sebbah, M. Stoytchev, and A. Z. Genack, "Delay-time statistics for diffuse waves", Phys. Rev. E 59, 7166 (1999). p.30, 40, and 41.

[96] A. Z. Genack, P. Sebbah, M. Stoytchev, and B. A. van Tiggelen, "Statistics of wave dynamics in random media", Phys. Rev. Lett. 82, 715 (1999). - p.30.

[97] P. M. Johnson, A. Imhof, B. P. J. Bret, J. G. Rivas, and A. LAGENDIJK, "Time-resolved pulse propagation in a strongly scattering material", Phys. Rev. E 68, 016604 (2003). — p.30 and 33.

[98] J. W. Goodman, Statistical Optics, John Wiley \& Sons (2000). — p.30, 57, and 78 .

[99] I. M. Vellekoop, P. Lodahl, and A. LagendiJK, "Determination of the diffusion constant using phase-sensitive measurements", Phys. Rev. E 71, 056604 (2005). - p.31, 73, 81, and 83.

[100] F. Lemoult, G. Lerosey, J. de Rosny, and M. Fink, "Manipulating spatiotemporal degrees of freedom of waves in random media", Phys. Rev. Lett. 103, 173902 (2009). - p.31, 37, 42, and 110.

[101] F. van Beijnum, E. G. van Putten, A. LagendiJk, and A. P. Mosk, 
"Frequency bandwidth of light focused through turbid media", Opt. Lett. 36, 373 (2011). - p.31 and 118.

[102] R. Trebino, Frequency-Resolved Optical Gating: The Measurement of Ultrashort Laser Pulses, Springer (2000). - p.32.

[103] L. Lepetit, G. ChÉriaux, and M. Joffre, "Linear techniques of phase measurement by femtosecond spectral interferometry for applications in spectroscopy", J. Opt. Soc. Am. B 12, 2467 (1995). — p.32.

[104] H. Gersen, J. P. Korterik, N. F. van Hulst, and L. Kuipers, "Tracking ultrashort pulses through dispersive media: Experiment and theory", Phys. Rev. E 68, 026604 (2003). - p.33.

[105] C. Rewitz, T. Keitzl, P. Tuchscherer, S. Goetz, P. Geisler, G. Razinskas, B. Hecht, and T. Brixner, "Spectral-interference microscopy for characterization of functional plasmonic elements", Opt. Express 20, 14632 (2012). - p.33.

[106] A. Tajalli, D. J. McCabe, D. R. Austin, I. A. Walmsley, and B. ChaTEL, "Characterization of the femtosecond speckle field of a multiply scattering medium via spatio-spectral interferometry", Journal of the Optical Society of America B 29, 1146 (2012). - p.33.

[107] E. P. Ippen and C. V. Shank, Ultrashort Light Pulses, Springer-Verlag, Berlin (1977). - p.33.

[108] J.-C. M. Diels, J. J. Fontaine, I. C. McMichael, and F. Simoni, "Control and measurement of ultrashort pulse shapes (in amplitude and phase) with femtosecond accuracy", Appl. Optics 24, 1270 (1985). — p.33, 72, 73, and 98.

[109] O. Katz, E. Small, Y. Bromberg, and Y. Silberberg, "Focusing and compression of ultrashort pulses through scattering media", Nat. Photon. 5, 372 (2011). - p.34, 70, 90, and 110.

[110] S. W. Hell and J. Wichmann, "Breaking the diffraction resolution limit by stimulated emission: stimulated-emission-depletion fluorescence microscopy", Opt. Lett. 19, 780 (1994). — p.37.

[111] W. R. Zipfel, R. M. Williams, and W. W. WebB, "Nonlinear magic: multiphoton microscopy in the biosciences", Nat. Biotech. 21, 1369 (2003). - p.37 and 43.

[112] E. N. Glezer, M. Milosavljevic, L. Huang, R. J. Finlay, T.-H. Her, J. P. Callan, and E. Mazur, "Three-dimensional optical storage inside transparent materials", Opt. Lett. 21, 2023 (1996). — p.37.

[113] A. H. ZEWAIL, "Femtochemistry: Atomic-scale dynamics of the chemical bondt", J. Phys. Chem. A 104, 5660 (2000). — p.37.

[114] J. SHAH, Ultrafast spectroscopy of semiconductors and semiconductor nanostructures, Springer (1999). — p.37 and 90.

[115] A. F. Koenderink and W. L. Vos, "Light exiting from real photonic band gap crystals is diffuse and strongly directional", Phys. Rev. Lett. 91, 213902 (2003). - p.37.

[116] J. Dainty, Laser Speckle and Related Phenomena, Springer (1984). — p.37.

[117] A. Z. Genack and J. M. Drake, "Relationship between optical intensity, fluctuations and pulse propagation in random media", Europhys. Lett. 11, 331 (1990). — p.37, 40, 51, and 90. 
[118] M. Rueckel, J. A. Mack-Bucher, and W. Denk, "Adaptive wavefront correction in two-photon microscopy using coherence-gated wavefront sensing", Proc. Natl. Acad. Sci. U.S.A. 103, 17137 (2006). — p.38.

[119] J. M. Dela Cruz, I. Pastirk, M. Comstock, V. V. Lozovoy, and M. DAnTus, "Use of coherent control methods through scattering biological tissue to achieve functional imaging", Proc. Natl. Acad. Sci. U.S.A. 101, 16996 (2004). - p.38.

[120] E. J. McDowell, M. Cui, I. M. Vellekoop, V. Senekerimyan, Z. YAQOOB, and C. YANG, "Turbidity suppression from the ballistic to the diffusive regime in biological tissues using optical phase conjugation", J. Biomed. Opt. 15, 025004 (2010). - p.38 and 43.

[121] T. Cizmar, M. Mazilu, and K. Dholakia, "In situ wavefront correction and its application to micromanipulation", Nat. Photon. 4, 388 (2010). — p.38, 70 , and 90 .

[122] S. H. Simon, A. L. Moustakas, M. Stoytchev, and H. Safar, "Communication in a disordered world", Phys. Today 54, 38 (2001). — p.38.

[123] O. L. Muskens and A. LagendiJK, "Broadband enhanced backscattering spectroscopy of strongly scatteringmedia", Opt. Express 16, 1222 (2008). p.39 and 51.

[124] M. Sandtke, R. J. P. Engelen, H. Schoenmaker, I. Attema, H. Dekker, I. Cerjak, J. P. Korterik, F. B. Segerink, and L. Kuipers, "Novel instrument for surface plasmon polariton tracking in space and time", Rev. Sci. Instrum. 79, 013704 (2008). - p.39 and 45.

[125] D. Yelin, D. Meshulach, and Y. Silberberg, "Adaptive femtosecond pulse compression", Opt. Lett. 22, 1793 (1997). — p.43.

[126] C. Loo, A. Lowery, N. Halas, J. West, and R. Drezek, "Immunotargeted nanoshells for integrated cancer imaging and therapy", Nano Letters $\mathbf{5}$, 709 (2005). - p.43.

[127] P. SebBah, Waves and Imaging Through Complex Media, Springer (2001). - p.43.

[128] A. Tourin, A. Derode, and M. Fink, "Dynamic time reversal of randomly backscattered acoustic waves", Europhys. Lett. 47, 175 (1999). — p.43.

[129] P. W. Anderson, "Absence of diffusion in certain random lattices", Physical Review 109, 1492 (1958). — p.43.

[130] S. E. Skipetrov and B. A. van Tiggelen, "Dynamics of anderson localization in open 3D media", Phys. Rev. Lett. 96, 043902 (2006). — p.43.

[131] B. D. Hughes, Random Walks and Random Environments: Random walks, Oxford University Press (1995). — p.50.

[132] D. Young and N. Beaulieu, "The generation of correlated rayleigh random variates by inverse discrete fourier transform", IEEE T. Commun. 48, 1114 (2000). - p.57.

[133] D. Meshulach, D. Yelin, and Y. Silberberg, "Adaptive ultrashort pulse compression and shaping", Opt. Commum. 138, 345 (1997). — p.59, 61, 117, and 121.

[134] I. M. Vellekoop, M. Cui, and C. YANG, "Digital optical phase conjugation of fluorescence in turbid tissue", Appl. Phys. Lett. 101, 081108 (2012). — p.70. 
[135] J. Aulbach, B. Guonaj, P. M. Johnson, A. P. Mosk, and A. Lagendijk, "Control of light transmission through opaque scattering media in space and time", Phys. Rev. Lett. 106, 103901 (2011). — p.70, 83, 84, 90, 92, 94, 95, 110 , and 120 .

[136] J. Aulbach, A. Bretagne, M. Fink, M. Tanter, and A. Tourin, "Optimal spatiotemporal focusing through complex scattering media", Phys. Rev. E 85, 016605 (2012). — p.70.

[137] C.-L. Hsieh, Y. Pu, R. Grange, and D. Psaltis, "Second harmonic generation from nanocrystals under linearly and circularly polarized excitations", Opt. Express 18, 11917 (2010). - p.70, 76, and 77.

[138] L. Le Xuan, S. Brasselet, F. Treussart, J.-F. Roch, F. Marquier, D. Chauvat, S. Perruchas, C. Tard, and T. Gacoin, "Balanced homodyne detection of second-harmonic generation from isolated subwavelength emitters", Appl. Phys. Lett. 89, 121118 (2006). — p.70.

[139] P. C. RAY, "Size and shape dependent second order nonlinear optical properties of nanomaterials and their application in biological and chemical sensing", Chem. Rev. 110, 5332 (2010). — p.70.

[140] R. Grange, T. Lanvin, C.-L. Hsieh, Y. Pu, and D. Psaltis, "Imaging with second-harmonic radiation probes in living tissue", Biomed. Opt. Express 2, 2532 (2011). - p.70.

[141] C.-L. Hsieh, R. Grange, Y. Pu, and D. Psaltis, "Three-dimensional harmonic holographic microcopy using nanoparticles as probes for cell imaging", Opt. Express 17, 2880 (2009). - p.72 and 113.

[142] J. D. Jackson, Classical electrodynamics, Wiley (1975). — p.74.

[143] R. W. Boyd, Nonlinear Optics, Academic Press (2008). — p.76.

[144] S. Roke and G. Gonella, "Nonlinear light scattering and spectroscopy of particles and droplets in liquids", Annu. Rev. Phys. Chem. 63, 353 (2012). - p.77.

[145] E. G. van Putten, A. Lagendijk, and A. P. Mosk, "Optimal concentration of light in turbid materials", J. Opt. Soc. Am. B 28, 1200 (2011). - p.77.

[146] B. Richards and E. Wolf, "Electromagnetic diffraction in optical systems. II. structure of the image field in an aplanatic system", Proc. R. Soc. Lond. A 253, 358 (1959). - p.77.

[147] I. Vellekoop and A. Mosk, "Phase control algorithms for focusing light through turbid media", Opt. Commum. 281, 3071 (2008). — p.83 and 98.

[148] D. B. Conkey, A. N. Brown, A. M. Caravaca-Aguirre, and R. PiesTUN, "Genetic algorithm optimization for focusing through turbid media in noisy environments", Opt. Express 20, 4840 (2012). — p.83.

[149] M. D. Leistikow, A. P. Mosk, E. Yeganegi, S. R. Huisman, A. LAGENDIJK, and W. L. Vos, "Inhibited spontaneous emission of quantum dots observed in a 3D photonic band gap", Phys. Rev. Lett. 107, 193903 (2011). - p.90.

[150] F. Lemoult, G. Lerosey, J. de Rosny, and M. Fink, "Resonant metalenses for breaking the diffraction barrier", Phys. Rev. Lett. 104, 203901 (2010). - p.90.

[151] J. Aulbach, B. Guonaj, P. Johnson, and A. Lagendijk, "Spatiotempo- 
ral focusing in opaque scattering media by wave front shaping with nonlinear feedback", Opt. Express 20, 29237 (2012). — p.90 and 92.

[152] P. Jiang, J. F. Bertone, K. S. Hwang, and V. L. Colvin, "Single-crystal colloidal multilayers of controlled thickness", Chem. Mater. 11, 2132 (1999). - p.95.

[153] J. H. J. ThiJssen, Characterization of photonic colloidal crystals in real and reciprocal space, Ph.D. thesis, University of Utrecht (2007). — p.95.

[154] A. Hartsuiker and W. L. Vos, "Structural properties of opals grown with vertical controlled drying", Langmuir 24, 4670 (2008). — p.95.

[155] R. Rengarajan, D. Mittleman, C. Rich, and V. Colvin, "Effect of disorder on the optical properties of colloidal crystals", Phys. Rev. E 71, 016615 (2005). - p.95.

[156] M. Tanter, J.-F. Aubry, J. Gerber, J.-L. Thomas, and M. Fink, "Optimal focusing by spatio-temporal inverse filter. i. basic principles", J. Acoust. Soc. Am. 110, 37 (2001). - p.110 and 112.

[157] E. Herbert, M. Pernot, G. Montaldo, M. Fink, and M. Tanter, "Energy-based adaptive focusing of waves: application to noninvasive aberration correction of ultrasonic wavefields", IEEE Ultr. Ferro. Freq. Cont. 56, 2388 (2009). — p.110, 113, and 114.

[158] B. Larrat, M. Pernot, G. Montaldo, M. Fink, and M. Tanter, "MRguided adaptive focusing of ultrasound", IEEE Ultr. Ferro. Freq. Cont. 57, 1734 (2010). - p.110.

[159] D. J. McCabe, A. Tajalli, D. R. Austin, P. Bondareff, I. A. WalmSley, S. Gigan, and B. Chatel, "Spatio-temporal focusing of an ultrafast pulse through a multiply scattering medium", Nat. Comm. 2, 447 (2011). — p.110.

[160] T. G. Leighton, The acoustic bubble, Elsevier (1994). — p.113.

[161] N. De Jong, M. Emmer, C. T. Chin, A. Bouakaz, F. Mastik, D. Lohse, and M. Versluis, "Compression-Only" behavior of phospholipid-coated contrast bubbles", Ultrasound Med. Biol. 33, 653 (2007). — p.113.

[162] A. Derode, A. Tourin, and M. Fink, "Random multiple scattering of ultrasound. II. is time reversal a self-averaging process?", Phys. Rev. E 64, 036606 (2001). - p.118. 